\title{
MEGASONIC CLEANING OF WAFERS IN ELECTROLYTE SOLUTIONS: POSSIBLE ROLE OF ELECTRO- ACOUSTIC AND CAVITATION EFFECTS
}

\author{
by \\ Manish Keswani \\ Copyright (C) Manish Keswani 2008 \\ A Dissertation Submitted to the Faculty of the \\ DEPARTMENT OF CHEMICAL AND ENVIRONMENTAL ENGINEERING \\ In Partial Fulfillment of the Requirements \\ For the Degree of \\ DOCTOR OF PHILOSOPHY \\ WITH A MAJOR IN CHEMICAL ENGINEERING \\ In the Graduate College \\ THE UNIVERSITY OF ARIZONA
}

2008 


\section{THE UNIVERSITY OF ARIZONA GRADUATE COLLEGE}

As members of Dissertation Committee, we certify that we have read the dissertation prepared by Manish Keswani

entitled Megasonic Cleaning of Wafers in Electrolyte Solutions: Possible Role of Electroacoustic and Cavitation Effects

and recommend that it be accepted as fulfilling the dissertation requirement for the Degree of Doctor of Philosophy

Date: 04/15/2008

Srini Raghavan

Date: $04 / 15 / 2008$

Pierre Deymier

Date: $04 / 15 / 2008$

James Farrell

Date: 04/15/2008

Paul Blowers

Date: $04 / 15 / 2008$

Steven Verhaverbeke

Final approval and acceptance of this dissertation is contingent upon the candidate's submission of the final copies of the dissertation to the Graduate College.

I hereby certify that I have read this dissertation prepared under my direction and recommend that it be accepted as fulfilling the dissertation requirement.

Dissertation Director: Srini Raghavan

Date: 04/15/2008 


\section{STATEMENT BY AUTHOR}

This dissertation has been submitted in partial fulfillment of requirements for an advanced degree at The University of Arizona and is deposited in the University Library to be made available to borrowers under rules of the Library.

Brief quotations from this dissertation are allowable without special permission, provided that accurate acknowledgment of source is made. Requests for permission for extended quotation from or reproduction of this manuscript in whole or in part may be granted by the copyright holder.

SIGNED: Manish Keswani 


\section{ACKNOWLEDGMENTS}

First and foremost, I would like to sincerely thank my advisor and mentor, Prof. Srini Raghavan for his valuable guidance and endless support in the completion of this dissertation. I have been highly fortunate to have a wonderful advisor who gave me the freedom to explore on my own, and at the same time gave the guidance to recover when my steps faltered. His infectious enthusiasm and unlimited zeal have been major driving forces through my graduate career. He truly provided me with an experience that very few live through and I will forever be indebted to him for that.

A very special and heartfelt thanks to Prof. Pierre Deymier for generously sharing his wisdom, talent and knowledge and guiding me in my research. His constructive criticisms at different stages of my research were thought-provoking and they helped me focus my ideas. Prof. James Farrell, the finest instructor, and mentor, I have had the privilege of knowing in all my years. Your instruction never failed to ignite a curiosity in me to learn more, to question, and challenge both myself and others. I would like to give my deepest appreciation to Prof. Paul Blowers for being so approachable and accommodating. Thank you so much for all your help and for answering my many questions in your cheerful and unperturbed manner. I owe my most sincere gratitude to Dr. Steven Verhaverbeke for his valuable advice, donations and friendly help. His important discussions around my work and interesting explorations have been very helpful for this study. I would like to also thank all of the committee members for spending their precious time to read this dissertation and for serving on my committee.

I wish to express my warm and sincere thanks to Prof. Shadman, Prof. Baygents, Prof. Kundu, Prof. Guzman, Prof. Serth, Prof. Pilehvari and Prof. Al-Sadoon for giving me a solid foundation in knowledge based on which I could come this far. I also owe my gratitude to the following people who have made this dissertation possible and because of whom my graduate experience has been one that I will cherish forever: Shariq Siddiqui (a great friend who always gave me support), Chemical Engineering and Material Science and Engineering faculty and staff, Nandini Venkataraman (special thanks for helping me in my defense presentation slides), Rose Myers, Sudib Mishra, Viraj Pandit, Hrishi Shende, Ashok Muthukumaran, Jovanny Gonzales, Ting Sun, Rumin Zhang, Ken Gaines, Kendra Gaines, Blanche Swyers, Mark Beck (for donation of megasonic tank), Buddy Curtis (for providing help with the oscilloscope and Labview set up), Gary Fleming, Krishna Muralidharan, Rachel Morrish, Esko, Fee Li, Kedar Dhane, Harpreet Juneja, Prakash Bakhru (uncle), Deepa and all my present and past colleagues.

A strong family support is critical when doing any type of work. Without prayers, well wishes and love of my parents, Mr. Hardas Keswani and Mrs. Dropadi Keswani, my brother Vinay, my sister Guddu, and my family back in India (Deepak and Kishore uncle, Asha and Mohini aunty, Rani bua, Dharamdas uncle, Padam, Monty, and Warora family) this work would not have been possible. I am especially grateful to my wife and best friend Pooja for her unconditional love and patience and for helping me keep my life in proper perspective and balance. I want to thank my son, Raj, who is my joy and I am truly blessed to have him in my life. Finally, I would like to thank God for everything. 
I would like to dedicate this dissertation to those who have influenced my life most, namely:

My parents (Hardas and Dropadi), wife (Pooja) and my advisor Prof. Srini Raghavan 
TABLE OF CONTENTS

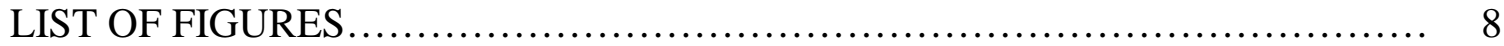

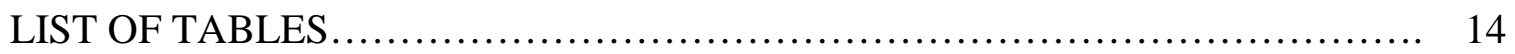

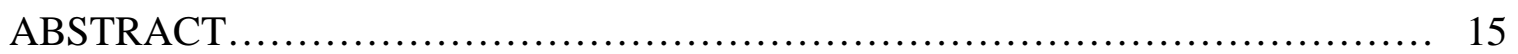

CHAPTER 1: INTRODUCTION............................................ 17

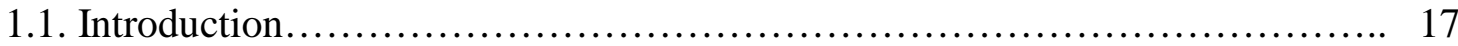

1.2. Contaminants.......................................................... 21

1.3. Wet and Dry Cleaning Methods.............................................. 24

1.4. Wet Cleaning Chemical Systems...................................... 28

1.5. Wafer Cleaning Techniques.......................................... 32

1.5.1. Immersion Cleaning.............................................. 33

1.5.2. Centrifugal Spraying............................................... 34

1.5.3. Brush Scrubbing ................................................ 37

1.5.4. High Pressure Fluid Jet Cleaning................................... 39

1.5.5. Megasonic Cleaning.................................................. 41

1.6. Research Objectives................................................ 46

CHAPTER 2: MEGASONICS.................................................... 47

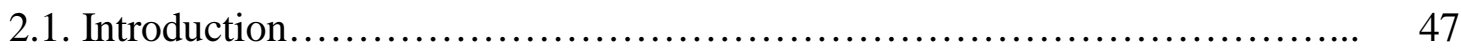

2.2. Cleaning Mechanisms.................................................. 51

2.3. Forces on a Particle in a Megasonic Field................................. 55

2.3.1. Van der Waals Forces........................................... 56

2.3.2. The Double-Layer Interactions.................................... 61

2.3.3. Forces Due to Electro-acoustic Effects in a Megasonic Field............. 68

2.4. Wave Acoustics..................................................... 83

2.4.1. Sound Wave Propagation......................................... 84

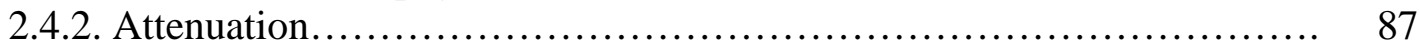

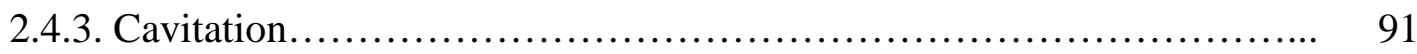

2.4.3.1. Introduction................................................ 91

2.4.3.2. Stable Cavitation.............................................. 92

2.4.3.3. Transient Cavitation................................................ 96

2.4.3.4. Shock Wave and Fluid Jet Formation............................. 107

2.4.4. Sonoluminescence.................................................. 114

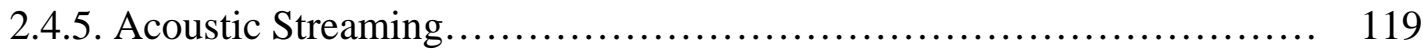

2.4.5.1. Eckart Streaming......................................... 120

2.4.5.2. Schlichting Streaming and Microstreaming....................... 122

CHAPTER 3: MATERIALS AND METHODS .............................. 125

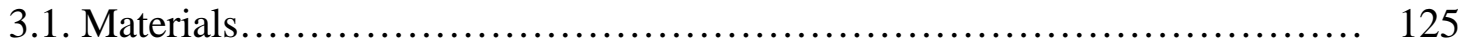

3.2. Megasonic System.................................................. 125

3.3. Particle Size Measurements.......................................... 128 
TABLE OF CONTENTS - Continued

3.4. Zeta Potential Measurements................................................ 129

3.5. Wafer Precleaning, Control Deposition and Cleaning...................... 133

3.6. Cavitation Measurements................................................ 137

3.7. Hydrophone Measurements......................................... 139

CHAPTER 4: RESULTS AND DISCUSSION ............................... 140

4.1. Zeta Potential Measurements of Plain and Aminated Silica Particles.......... 140

4.2. Megasonic Cleaning in Potassium Chloride Solutions...................... 142

4.2.1. Possible Role of Electro-acoustic Effects on Removal of Silica Particles

from Wafer Surfaces......................................... 147

4.2.1.1. Estimation of Zeta Potential from the Surface Charge Density of Aminated Silica Particles...................................... 151

4.2.1.2. Effect of Ionic Strength on Removal Force....................... 157

4.2.2. Effect of Ionic Strength on Cavitation.............................. 162

4.2.2.1. Modulation of Sound Wave Pressure Amplitude.................. 162

4.2.2.2. Experimental Investigation of Higher Concentrations of Potassium Chloride on Sound Wave Pressure Amplitude and Bubble Size

Distribution................................................. 164

4.2.2.2.1. Prediction of Transient Cavities from Bubble Size Distribution.. 167

4.2.2.3. Effect of Pressure Amplitude on Sonoluminescence................. 170

4.2.3. Effect of Pressure Amplitude on Removal of Silica Particles in Higher

Concentrations of Potassium Chloride Solutions...................... 171

4.2.4. Effect of Temperature on Removal of Aminated Silica Particles.......... 173

4.2.5. Effect of Solution $\mathrm{pH}$ on Removal of Aminated Silica Particles.......... 179

4.2.6. Effect of Cleaning Time on Removal of Aminated Silica Particles....... 183

4.2.7. Use of Other Chloride Based Electrolytes on Removal of Aminated Silica Particles.................................................... 184

CHAPTER 5: CONCLUSIONS AND FUTURE WORK......................... 191

5.1. Conclusions......................................................... 191

5.2. Future Work ...................................................... 193

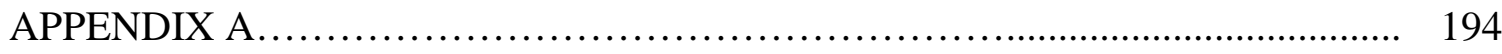

A.1. Derivation of Streaming Equation...................................... 194

A.1.1. Eckart Streaming Velocity in a Megasonic Cleaning Tank.............. 197

A.2. Wilcoxon Matched Pairs Signed Rank Test................................ 201

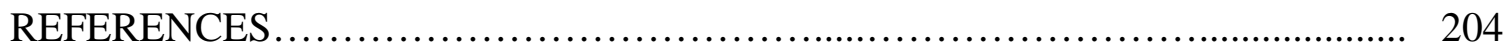




\section{LIST OF FIGURES}

Figure 1.1: Effect of defect density and chip area on percent yield at different defect distribution [Koehler and Beadle, 1985].

Figure 1.2: Surface preparation technology requirements $[1.3] \ldots \ldots \ldots \ldots \ldots \ldots \ldots \ldots .20$

Figure 1.3: Schematic of killer particle on wafer surface during formation of heavily doped buried layers beneath the active devices............................ 22

Figure 1.4: Contaminated silicon wafer with different types of impurities [1.5] ..... 23

Figure 1.5: Wafer cleaning sequence typically used in semiconductor

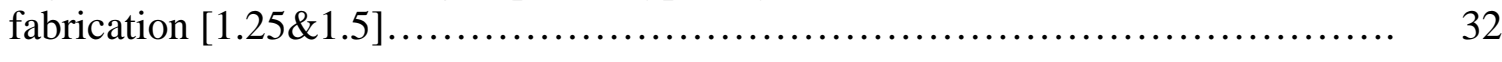

Figure 1.6: Schematic showing immersion cleaning of wafers.................... 34

Figure 1.7: Single wafer spray tool (SEZ DaVinci) ........................... 36

Figure 1.8: Schematic of brush scrubbing process $[1.29] \ldots \ldots \ldots \ldots \ldots \ldots \ldots \ldots \ldots \ldots . \ldots \ldots$

Figure 1.9: Schematic of high pressure fluid jet cleaning system.................. 40

Figure 1.10: Schematic showing particle removal mechanisms in megasonic

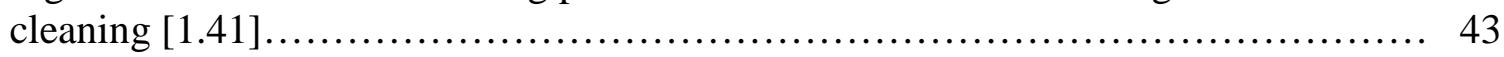

Figure 1.11: Damage of test structures (silicon fins on oxide surface) during megasonic cleaning (Courtesy of Prof. Raghavan).............................. 45

Figure 2.1: Spatial variation in cavitation in the megasonic cleaning tank [2.5]...... 49

Figure 2.2: Schematic showing cleaning mechanisms in an immersion type megasonic

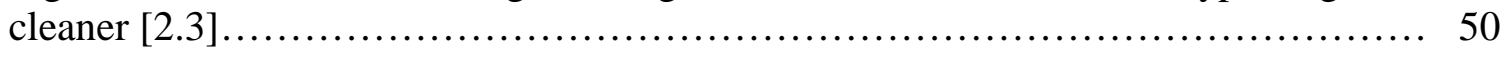

Figure 2.3: The different forces involved in rolling removal mechanism [1.44]...... 55

Figure 2.4: Non-retarded potential energy between pairs of solid surfaces of

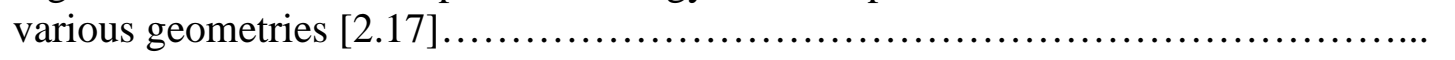

Figure 2.5: Schematic representation of different potentials associated with a

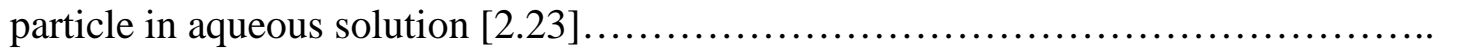




\section{LIST OF FIGURES - Continued}

Figure 2.6: Schematic of Debye effect showing formation of IVP due to the difference in displacements of the cations and the anions [2.33].

Figure 2.7: Schematic of relaxation force on an ion displaced from its equilibrium in an acoustic field [2.29]

Figure 2.8: a) Hydrodynamic drag on a moving ion due to the surrounding ions b) Net diffusion force due to regions of positive and negative ions [2.29] .............. 74

Figure 2.9: The decay in pressure amplitude of sound wave with distance in a viscous medium.

Figure 2.10: Radius of oscillating bubbles versus frequency for large bubbles (surface tension forces insignificant)

Figure 2.11: Non-linear oscillation of stable gas bubbles showing ultraharmonics of the driving harmonics [2.49]

Figure 2.12: Blake threshold pressure for water and a mixture of ethylene glycol and ethanol as a function of initial bubble radius [2.52].

Figure 2.13: The change in size of a gas bubble during its growth and collapse [2.55]......

Figure 2.14: Schematic of bubble collapse [1.41]

100

Figure 2.15: The variation of maximum bubble size with externally applied pressure, initial radius of bubble and frequency of acoustic wave [2.54]

Figure 2.16: Blake's threshold, stable threshold and transient threshold for air saturated water at $20 \mathrm{KHz}$ [2.57]

Figure 2.17: Liquid velocity as a function of distance from bubble center after specified elapsed time [2.60].

Figure 2.18: Effect of source pressure amplitude and initial bubble radius on liquid velocity at shock wave front [2.60]

Figure 2.19: Effect of liquid surface tension and bubble radius on liquid velocity at shock wave front [2.60]. 


\section{LIST OF FIGURES - Continued}

Figure 2.20: Collapse of a spherical bubble initially in contact with solid boundary [2.61]

Figure 2.21: Collapse of a spherical bubble initially in contact with solid boundary [2.61]

Figure 2.22: Schematic of Eckart streaming velocity profile in a megasonic cleaning tank.

Figure 2.23: Maximum Eckart streaming velocity at different transducer intensities and sound wave frequencies in a megasonic tank

Figure 2.24: Schlichting streaming [2.76]

Figure 2.25: Sequence of microstreaming patterns at different bubble surface velocities [2.76].....

Figure 3.1: Schematic of transducer bonding details

Figure 3.2: Schematic of megasonic system.

Figure 3.3: FESEM image of aminated silica particles.

Figure 3.4: FESEM image of plain silica particles.

Figure 3.5: Size distribution of aminated silica particles measured using Coulter N4 Plus particle analyzer.

Figure 3.6: Size distribution of plain silica particles measured using Coulter N4 Plus particle analyzer.

Figure 3.7: Image of 150-mm Si wafer after pre-cleaning step using SURFSCAN 5500.

Figure 3.8: Image of 150-mm Si wafer deposited with aminated silica particles $(367 \pm 20 \mathrm{~nm})$ using SURFSCAN 5500.

Figure 3.9: Histogram showing distribution of particles in different bin sizes after pre-cleaning step. 


\section{LIST OF FIGURES - Continued}

Figure 3.10: Histogram showing distribution of aminated silica particles $(367 \pm 20 \mathrm{~nm})$ in different bin sizes after deposition step

Figure 3.11: Schematic of the cavitation probe [3.4]

Figure 4.1: ZP of plain and aminated silica particles

Figure 4.2: Removal of plain silica particles from wafer surface in $\mathrm{KCl}$ solution of different concentrations at $30{ }^{\circ} \mathrm{C}$ and power density of $0.077 \mathrm{~W} / \mathrm{cm}^{2} \ldots$

Figure 4.3: Removal of plain silica particles from wafer surface in $0.57 \mathrm{M} \mathrm{KCl}$ solution and DI water at $30{ }^{\circ} \mathrm{C}$.

Figure 4.4: Removal of aminated silica particles from wafers in $\mathrm{KCl}$ solutions of different concentrations.

Figure 4.5: Critical concentration of $\mathrm{KCl}$ at $30{ }^{\circ} \mathrm{C}$ for removal of aminated silica particles from wafer surface at different power densities.

Figure 4.6: Debye potential in $\mathrm{KCl}$ solutions of different concentration and power densities.

Figure 4.7: Schematic of back flow of ions in the case of aminated silica particle suspended in electrolyte solution subjected to acoustic field.

Figure 4.8: Schematic showing reduced back flow of ions for aminated silica particle adhered to a wafer surface immersed in an electrolyte solution subjected to acoustic field.

Figure 4.9: Surface potential of aminated silica particles at solution $\mathrm{pH}$ of 4.5 and temperature of $30{ }^{\circ} \mathrm{C}$.

Figure 4.10: Surface potential of aminated silica particles at solution $\mathrm{pH}$ of 6.0 and temperature of $30{ }^{\circ} \mathrm{C}$.

Figure 4.11: Zeta potential of aminated silica particles at solution $\mathrm{pH}$ of 6.0 and temperature of $30^{\circ} \mathrm{C}$.

Figure 4.12: Different combinations of $Q^{\prime}$ and $l$ that will satisfy a fixed value of dipole moment, $m$ 


\section{LIST OF FIGURES - Continued}

Figure 4.13: Magnitude of removal force, $F$, computed for fixed $m$ and different values of $l$, for transducer power density of $0.43 \mathrm{~W} / \mathrm{cm}^{2}$ and ionic strength of $3 \times 10^{-5} \mathrm{M}$.

Figure 4.14: A point center mass of charge $+Q$ surrounded by ionic cloud of radius $r$ ' and charge $-Q$

Figure 4.15: Shifting of ionic charge due to applied acoustic field.

Figure 4.16: Shifting of ionic charge at higher ionic strength in an acoustic field.... 160

Figure 4.17: Removal force as a function of displacement at ionic strengths of $0.0001 \mathrm{M}$ and $0.00003 \mathrm{M}$ and power densities of $0.43 \mathrm{~W} / \mathrm{cm}^{2}$ and $0.15 \mathrm{~W} / \mathrm{cm}^{2}$

Figure 4.18: Critical electrolyte concentration as a function of power density 162

Figure 4.19: Bulk modulus and density of solution at different $\mathrm{KCl}$ concentration at $25{ }^{0} \mathrm{C}$

Figure 4.20: Sound wave pressure amplitude in $\mathrm{KCl}$ solution of different concentration at power density of $2.17 \mathrm{~W} / \mathrm{cm}^{2}$ and solution temperature of $25^{0} \mathrm{C} \ldots$.

Figure 4.21: Hydrophone measurements in $\mathrm{KCl}$ solutions of different concentration and power densities.

Figure 4.22: Bubble size distribution in $\mathrm{KCl}$ solution of different concentrations at $30{ }^{\circ} \mathrm{C}$ and power density of $2.17 \mathrm{~W} / \mathrm{cm}^{2}$.

Figure 4.23: Cavitation in DI water and $\mathrm{KCl}$ solutions of two different concentrations for $10 \mathrm{~min}$ at $30{ }^{\circ} \mathrm{C}$ and power density of $2.17 \mathrm{~W} / \mathrm{cm}^{2}$.

Figure 4.24: Effect of temperature on mobilities of $\mathrm{K}^{+}$and $\mathrm{Cl}^{-}$ions in $\mathrm{KCl}$ solution of infinite dilution [4.7]

Figure 4.25: $\mathrm{SL}$ in $0.3 \mathrm{M} \mathrm{KCl}$ solution at power density of $0.43 \mathrm{~W} / \mathrm{cm}^{2}$ and temperatures of $30{ }^{\circ} \mathrm{C}$ and $40{ }^{\circ} \mathrm{C}$ for $10 \mathrm{~min}$.

Figure 4.26: Solution $\mathrm{pH}$ of $\mathrm{HCl}, \mathrm{NaOH}, \mathrm{NH}_{4} \mathrm{OH}$ and $\mathrm{KCl}$ at $33{ }^{\circ} \mathrm{C}$ as a function of ionic strengths. 


\section{LIST OF FIGURES - Continued}

Figure 4.27: Removal of aminated silica particles from wafer surfaces in solutions of different $\mathrm{pH}$ at megasonic power density of $0.43 \mathrm{~W} / \mathrm{cm}^{2}$ and temperature of $30{ }^{\circ} \mathrm{C}$.

Figure 4.28: Removal of aminated silica particles in solutions of different $\mathrm{pH}$ in the absence of megasonic field at $30^{\circ} \mathrm{C}$.

Figure 4.29: Removal of aminated silica particles in $10^{-3} \mathrm{M} \mathrm{KCl}$ solution at $0.103 \mathrm{~W} / \mathrm{cm}^{2}$ as a function of cleaning time.

Figure 4.30: Removal of aminated silica particles in solutions of different electrolytes at $0.43 \mathrm{~W} / \mathrm{cm}^{2}$ (Solution Conditions: $\mathrm{pH}=6.0$, Temperature $=30{ }^{0} \mathrm{C}$ )...

Figure 4.31: Removal of aminated silica particles in solutions of different electrolytes at $0.129 \mathrm{~W} / \mathrm{cm}^{2}$ (Solution Conditions: $\mathrm{pH}=6.0$, Temperature $=30^{0} \mathrm{C}$ ) $\ldots 186$

Figure 4.32: Removal of aminated silica particles in solutions of different electrolytes at $0.103 \mathrm{~W} / \mathrm{cm}^{2}$ (Solution Conditions: $\mathrm{pH}=6.0$, Temperature $=30{ }^{0} \mathrm{C}$ )... 186

Figure 4.33: Removal of aminated silica particles in solutions of different electrolytes at $0.077 \mathrm{~W} / \mathrm{cm}^{2}$ (Solution Conditions: $\mathrm{pH}=6.0$, Temperature $=30{ }^{0} \mathrm{C}$ )...

Figure 4.34: Debye potential in different electrolyte solutions at $0.43 \mathrm{~W} / \mathrm{cm}^{2}$ of megasonic power density at $30{ }^{\circ} \mathrm{C}$.

Figure A.1: Schematic for transducer in megasonic tank with different dimensions

Figure A.2: Maximum Eckart streaming velocity at different transducer intensities and sound wave frequency for open channel....

Figure A.3: Maximum Eckart streaming velocity at different transducer intensities and sound wave frequency for closed channel. 


\section{LIST OF TABLES}

Table 2.1: Hamaker constants for different materials [2.13, 2.15, \& 2.16].

58

Table 4.1: Effect of temperature on removal of aminated silica particles from wafer surface in $\mathrm{KCl}$ solutions of different concentrations and power densities.......

Table 4.2: Sample calculations for comparison between $\mathrm{NaCl}$ and $\mathrm{KCl}$ following

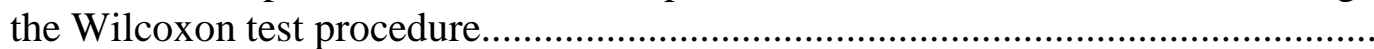

Table 4.3: Summary of performance ranking of electrolytes obtained after statistical analysis using Wilcoxon Matched-Pairs Signed Rank test.....................................

Table A.1: Tabled Critical Wilcoxon T values for $\mathrm{n}=9$ Signed Ranks.................... 203 


\begin{abstract}
Megasonic cleaning is routinely used in the semiconductor industry to remove particulate contaminants from wafer and mask surfaces. Cleaning is achieved through proper choice of chemical solutions, power density and frequency of acoustic field. Considerable work has been done to increase understanding of particle removal mechanisms in megasonic cleaning using different solution chemistries with varying ionic strengths. However, to date, the focus of all these studies of particle removal has been either cavitation or acoustic streaming.
\end{abstract}

The propagation of sound waves through a colloidal dispersion containing ions is known to result in the generation of two types of oscillating electric potentials, namely, Ionic Vibration Potential (IVP) and Colloid Vibration Potential (CVP). These potentials and their associated electric fields can exert forces on charged particles adhered to a surface, resulting in their removal. In addition, the pressure amplitude of the sound wave is also altered in solutions of higher ionic strengths, which can affect the cavitation process and further aid in the removal of particles from surfaces. To test the two hypotheses, investigations have been conducted on the feasibility of removal of charged particles from 150-mm Si wafers in electrolyte solutions of different ionic strengths irradiated with a megasonic field of different power densities. Cleaning experiments have been performed using potassium chloride $(\mathrm{KCl})$ as a model electrolyte and silica particles as model contaminant particles. The cleaning performance in $\mathrm{KCl}$ solution has been 
compared to that in other electrolytes solutions such as sodium chloride $(\mathrm{NaCl})$, cesium chloride $(\mathrm{CsCl})$ and lithium chloride $(\mathrm{LiCl})$. In order to characterize the cavitation events in $\mathrm{KCl}$ solutions, acoustic pressure and sonoluminescence measurements have been performed using hydrophone and cavitation probe respectively. The results indicate that particle removal efficiency (PRE) increases with $\mathrm{KCl}$ concentration and transducer power density and much lower power densities are required at higher $\mathrm{KCl}$ concentration for a comparable level of cleaning. Further, cleaning performances in $\mathrm{NaCl}$ and $\mathrm{CsCl}$ were found to be superior to those in $\mathrm{KCl}$ and $\mathrm{LiCl}$ solutions. Theoretical computations show that the removal forces due to CVP are much larger in magnitude than those due to IVP and are comparable to van der Waals adhesion forces. Pressure measurements show that ionic strength of the solution affects the cavitation process in $\mathrm{KCl}$ solutions of concentration greater than $0.01 \mathrm{M}$. 


\section{CHAPTER 1: INTRODUCTION}

\subsection{Introduction}

The invention of the Intergrated Circuit (IC) has brought with it a new revolution in today's world and dramatically changed our lives. The first bipolar transistor, a point contact type, was created by J. Bardeen, W. Brattain and W. Shockley in December 1947 at Bell Telephone Laboratories. Fundamentally, an IC or chip consists of several individual components such as transistors, resistors, capacitors, which are placed side by side and wired to form a complex structure capable of performing a particular circuit function. With the ever-growing need to improve the performance of electronic devices, there has been a continuous effort to reduce the size of these individual components and increase the chip sizes. This allows more components to be integrated in a chip, thus significantly enhancing the functioning of the integrated systems and keeping the cost low at the same time. In the 1960's, Gordon Moore, Intel cofounder, described an important trend in the history of computer hardware: that the number of transistors on a chip would double every two years without accounting for an increase in the chip size [1.1]. This trend has been preserved over the years and has led to continuous improvement in the computing power and development of high tech devices. One of the drivers behind this growth is the evolution of wafer cleaning technology that has maintained the contamination and defectivity levels within the required specifications. 
Wafer cleaning is an important step in fabrication of very large scale integration (VLSI) and ultra large scale integration (ULSI) silicon circuits in order to maintain their reliability and efficient performance. It is estimated that about fifty percent of yield losses in the IC industry is due to particle contamination [1.2]. Figure 1.1 shows the percent yield as a function of product of the defect density and chip area $\left(D_{0} A\right)$ at different values of variance of defect distribution $(\lambda)$. A three-parameter yield model, expressed in equation 1.1, was used to obtain the values of the percent yield.

$\%$ Yield $=\frac{1}{\left(1+\lambda D_{0} A\right)^{1 / \lambda}}$

The yield decreases rapidly with the product of defect density and the chip area and is lower for smaller variances. A smaller variance indicates that the defect distribution is more random, which decreases the probability of multiple defects on the same chip and hence reduces the percent yield. A continuous effort to improve this percent yield has led to not only an increase in the number of cleaning steps but also an imposition of more critical requirements by the International Technology Roadmap for Semiconductors (ITRS) on the tolerable size, concentration and number of contaminants on the front and back side of wafer surfaces (Figure 1.2). There is a strong need for the development of a cleaning process that is capable of efficiently removing the contaminants from the wafer without damaging the substrate, thereby improving the overall product yield. This dissertation identifies a new particle removal mechanism in a megasonic field to yield 
results that are expected to meet the ITRS cleaning requirements. The study includes experimental measurements and theoretical computations to characterize the effect of various solution variables on the cleaning efficiency and to enable the optimization of operating conditions for future cleaning applications.

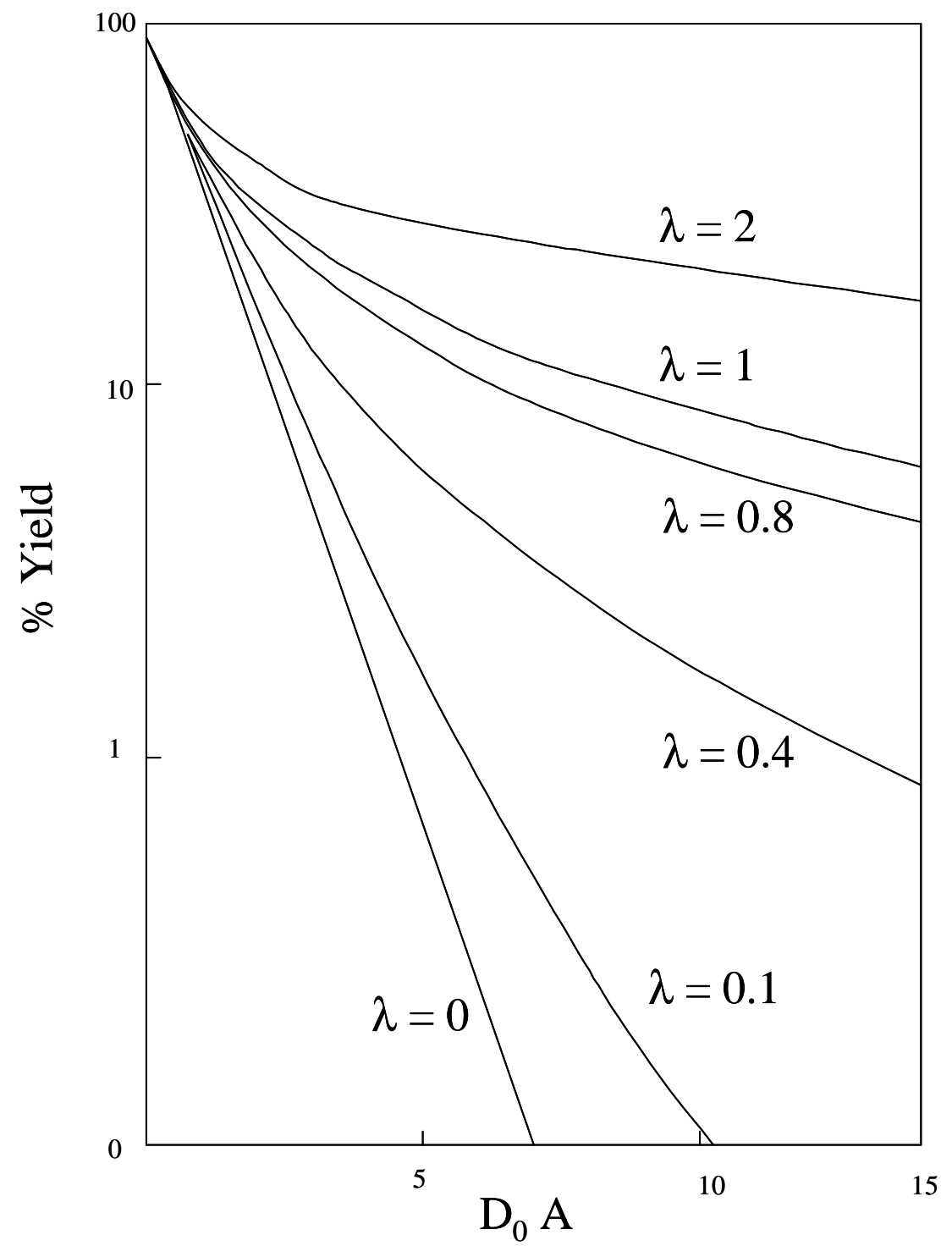

Figure 1.1: Effect of defect density and chip area on percent yield at different defect distribution [Koehler and Beadle, 1985] 


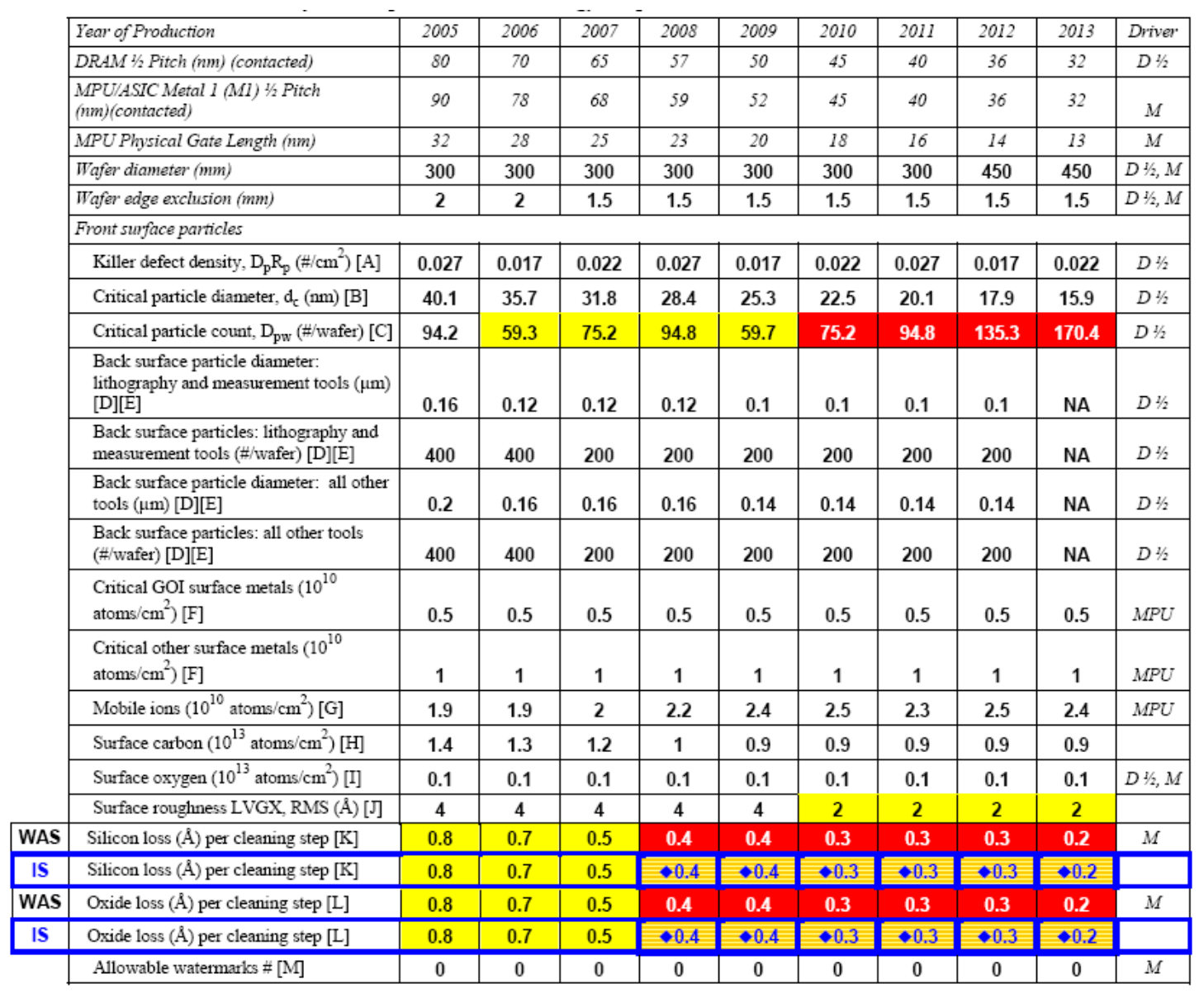

Manufacturable solutions exist, and are being optimized Manufacturable solutions are known Interim solutions are known Manufacturable solutions are NOT known

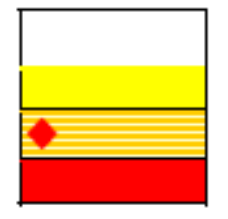

Figure 1.2: Surface preparation technology requirements [1.3] 
In the following sections, a brief review is given of the different types of contaminants that can exist on wafer surfaces and problems associated with them, followed by different chemical systems and techniques used for wafer cleaning with their advantages and limitations.

\subsection{Contaminants}

Four types of contaminations are commonly observed on substrates used in the semiconductor industry, namely particles, contaminant films, elemental metal or ionic species and gaseous contaminants. The various sources of particles known to industry are factory personnel, equipment, solid materials, liquid and gaseous chemicals, containers, air borne contaminants, piping and tubing, etc. Static charge build-up can be a critical mechanism for particle attraction but is often given less attention in industry. The ITRS roadmap dictates that by 2008 , the killer defect density, critical particle diameter and count must be $0.017 \# / \mathrm{cm}^{2}, 25.3 \mathrm{~nm}$ and $59.7 \# /$ wafer respectively for the front surface of a $300 \mathrm{~mm}$ wafer. [1.3]. A schematic of a killer particle on a wafer surface during formation of heavily doped buried layers beneath the active devices is shown in figure 1.3. The killer particle can mask the arsenic implantation in certain areas causing 'latchup' problems in operating CMOS circuits after fabrication.

Control of impurities in the form of nano particles is especially important during high temperature processing (thermal oxidation, diffusion, epitaxial growth) in which the 
small sized particles can diffuse easily to the wafer interior and cause electrical defects and device degradation [1.2]. These smaller particles are also more difficult to remove compared to larger particles due to a higher ratio of adhesion to removal forces. Particle formation in the films during deposition can cause pinholes, microvoids and microcracks.

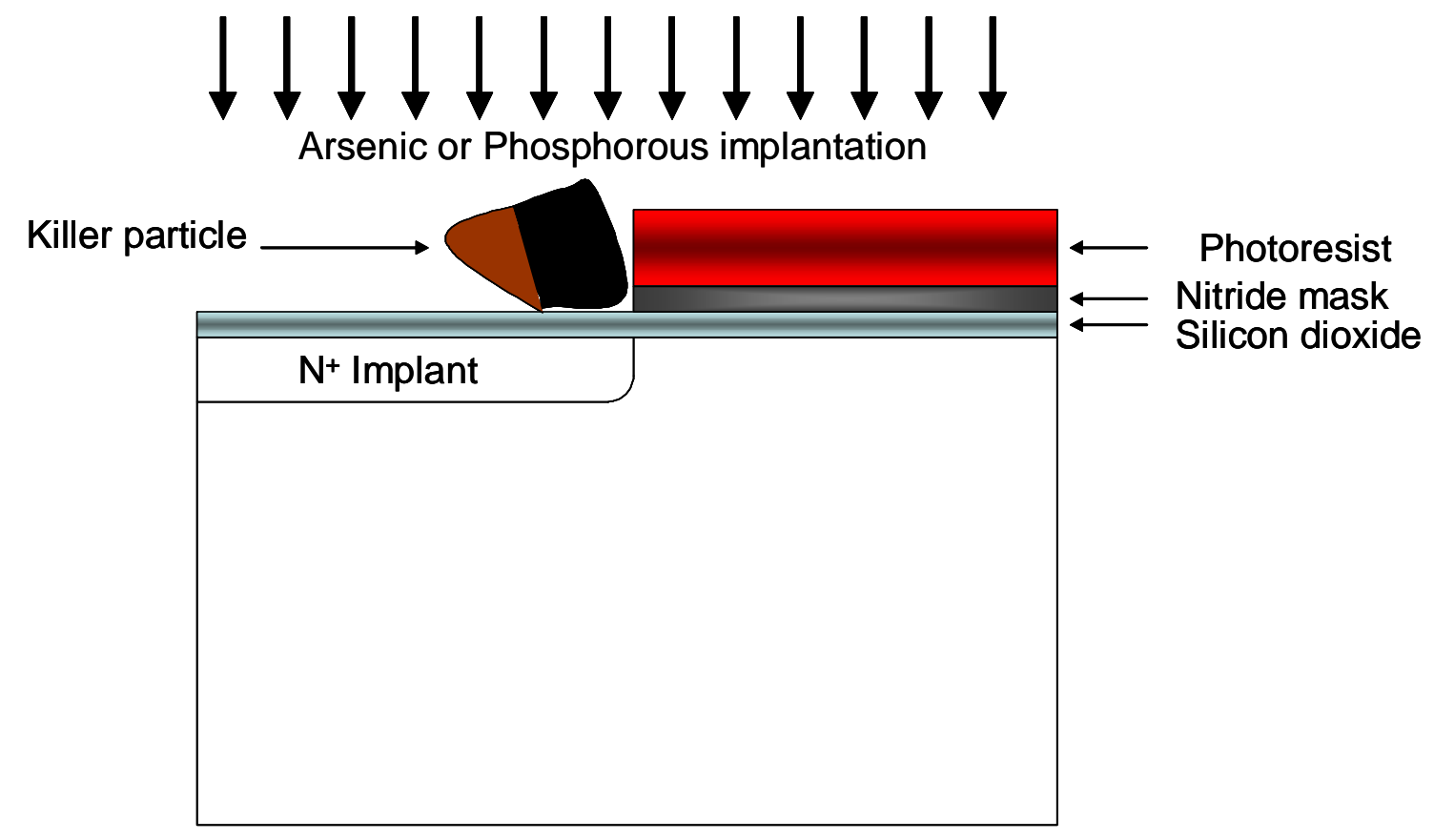

Figure 1.3: Schematic of killer particle on wafer surface during formation of heavily doped buried layers beneath the active devices

Contamination films consist of condensed organic vapors from lubricants, greases, photoresist, and solvent residues, as well as thin layers of metal oxides or hydroxides. These organic films, when present on the wafer surface, can alter adhesion properties between different layers and form reaction or decomposition products during processing. For example, an organic film, when heated in the absence of an oxidizing agent, can 
decompose into carbon which can react with silicon to form silicon carbide. This compound can provide a nucleation site for polycrystalline silicon growth during epitaxial deposition. Wafer carriers can be an important source of organic contamination which can reduce the breakdown field strength of the insulating $\mathrm{SiO}_{2}$ layer [1.4].

The ionic species, present either in the cationic or the anionic form, can physically or chemically adsorb on the wafer surface and change the surface characteristics. Metals like copper, gold or silver can be electroplated on the wafer surface in a strong reducing chemistry. Metal impurities and ionic species, often originating from the chemicals used in the processing, can cause a host of problems such as drift currents, unstable surface potential, twining dislocations and stacking faults. They can also affect minority-carrier lifetime, surface conduction and electrical device stability. A contaminated silicon wafer surface, with layers of oxide, photoresist, polysilicon, silicides, and metals, is shown in figure 1.4.

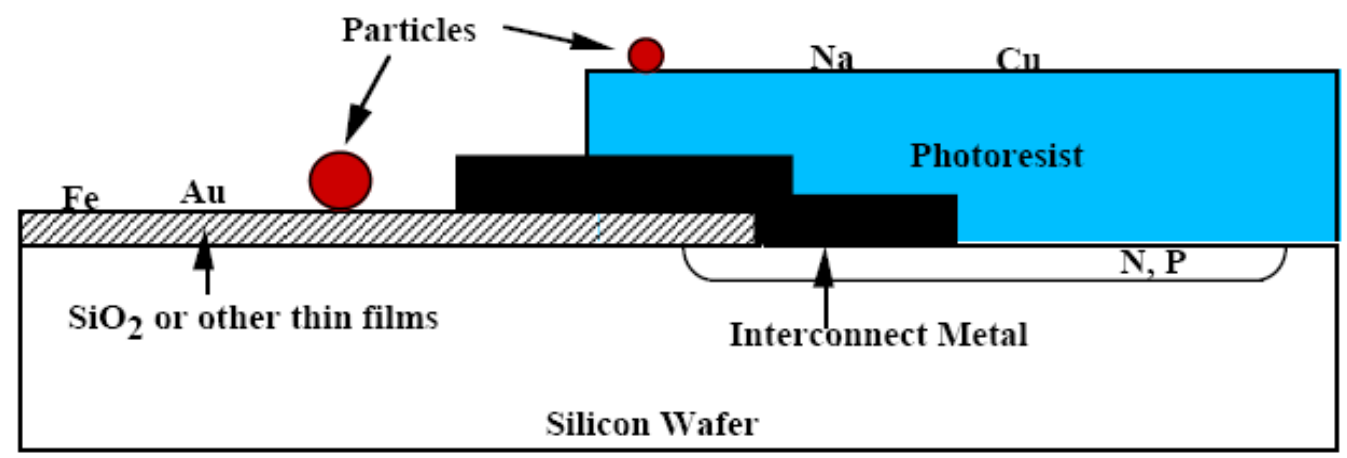

Figure 1.4: Contaminated silicon wafer with different types of impurities [1.5] 
It is obvious from the above discussion that the presence of impurities during wafer processing can adversely affect the device functioning and hamper the progress of the IC industry. Contamination can be controlled by reducing the level of impurities in the fabrication area by maintaining a clean environment and carrying out routine maintenance of the tools used for processing. Friction in tools, which is another source of contamination, can be reduced by providing proper lubrication and regular check-ups of the machines. Use of ultra pure water and clean chemicals is necessary to maintain a particle free environment during processing. It is much easier to prevent contamination than to remove it after it has occurred. However, it is not possible to avoid contamination in some cases where the design of the chemical process can itself be a source of contamination. For example, when siliane and oxygen or water vapor are used for chemical vapor deposition of silica, some reaction can occur away from the surface generating particles, which can later deposit on the wafer and act as contaminants.

\subsection{Wet and Dry Cleaning Methods}

The overall objective of today's wafer cleaning technology is to remove all types of contaminant impurities from the wafer surfaces without damaging or altering the substrate. The current technologies are based on the development of environmentally benign chemistries and techniques that will require minimal chemical usage and disposal in order to achieve the desired cleaning. Hydrogen peroxide based wet chemistries are

still the common form of cleaning techniques used for removal of contaminant films and 
particles from the wafer surfaces. However, its implementation has changed over the years from immersion cleaning to centrifugal spray cleaning, megasonic cleaning, and other methods.

Wafer cleaning methods can be broadly classified into wet and dry cleaning methods. Dry cleaning (or vapor phase cleaning) methods include jet scrubbing with dry carbon dioxide, cryogenic argon aerosol jet impingement, supercritical carbon dioxide, pulsed laser radiation, adhesive tape method, gas plasma cleaning, and others. Wet cleaning methods employ liquid based chemistries such as hydrofluoric acid solutions, sulfuric acid - peroxide mixtures, alkaline and acidic peroxide solutions (SC-1 and SC-2 solutions) and sulfuric acid-ozone mixtures that are more popular in industry compared to dry cleaning methods. The use of liquid in wet cleaning offers several advantages over the use of vapor/gas in dry cleaning for removal of particulate contaminants from the wafer surface. The dynamic pressure and drag force experienced by a particle held stationary in a moving fluid under a low Reynolds number hydrodynamics, as given by Stokes' analysis, are expressed in equations 1.2 and 1.3 [1.6]. Since the viscosity and density of the liquids are much larger than those of gases, the pressure and drag forces experienced by particles in liquids will be at least a few orders of magnitude larger than those in gases. In addition, a particle, under similar conditions, also experiences lower van der Waals forces of adhesion in liquids due to the larger value of dielectric constant of liquids compared to gases. 


$$
\begin{aligned}
& \mathrm{P}(r, \theta)=-\frac{3}{2} \frac{\eta U}{R}\left(\frac{R}{r}\right)^{2} \cos \theta \\
& F_{D}=6 \pi \eta U R\left[1+\frac{3}{8} \operatorname{Re}+\frac{9}{40} \operatorname{Re}^{2}\left(\ln \operatorname{Re}+\gamma+\frac{5}{3} \ln 2-\frac{323}{360}\right)+\frac{27}{80} \mathrm{Re}^{3} \ln \operatorname{Re}\right]
\end{aligned}
$$

where $\mathrm{P}$ and $F_{D}$ are the dynamic pressure and hydrodynamic drag force on the particle, $\theta$ is the angle between the negative $\mathrm{x}$-axis (opposite to the direction of flow of fluid) and a vector drawn at a point of interest, $U$ is the uniform velocity of the fluid far from the sphere, Re is the Reynolds number equal to $\frac{\rho U R}{\eta}, \rho$ and $\eta$, are the density and viscosity of the fluid, $R$, is the radius of the particle and $\gamma$ is the Euler's constant equal to 0.5772 .

The effect of a hydrodynamic boundary layer becomes critical when a fluid motion is considered on a flat wafer surface that is contaminated with particulate impurities. The formation of a boundary layer reduces the velocity of the fluid close to a surface and hence lessens the impact of inertial force that is available to remove the particles from the surface. Fluids with higher viscosity form a thicker boundary layer and hence reduce the impact of moving fluid to a greater extent. The boundary layer thicknesses for a laminar and turbulent flow of fluid over a flat plate are given by equations 1.4 and 1.5 respectively [1.7]. 


$$
\begin{aligned}
& \delta_{L}(x)=5\left(\frac{\eta x}{\rho U_{\infty}}\right) \\
& \delta_{T}(x)=\frac{0.14 \eta \operatorname{Re}_{x} G\left(\ln \operatorname{Re}_{x}\right)}{\rho U_{\infty} \ln \operatorname{Re}_{x}}
\end{aligned}
$$

where $\delta_{L}(x)$ and $\delta_{T}(x)$ are the laminar and turbulent boundary layer thicknesses, $U_{\infty}$ is the free stream velocity of fluid far away from the surface, $\operatorname{Re}_{x}$ is the Reynolds number, and $G\left(\ln \operatorname{Re}_{x}\right)$ is a weak function of $\ln \mathrm{Re}_{x}$ and is equal to 1.5 for $10^{5} \leq \operatorname{Re}_{x} \leq 10^{6}$

The ratio $\frac{\eta}{\rho}$ is approximately 15 times higher for air compared to water and so will be the laminar boundary layer thickness [1.7]. As a result, a particle on a wafer will experience lower velocity of fluid in air than in water. Similarly, since the velocity of a fluid decreases as one approaches the wafer surface, smaller particles experience lower hydrodynamic drag forces compared to the larger particles. This is also one of the reasons why smaller particles are more difficult to remove although they have weaker adhesion forces compared to larger particles.

Thus, one can clearly notice that wet cleaning has certain distinct advantages over dry cleaning and is thus more accepted in industry. However, there are also some disadvantages associated with wet cleaning which include the following [1.8]: 
1) In general, liquids have lower diffusivity than gases which limits their use for cleaning of high aspect ratio structures.

2) The liquid chemicals for wet cleaning supplied by vendors are not completely particle free. Therefore, although wet chemical methods are effective in particle removal, a few particles from the chemicals may be added to the wafer.

2) The wet chemical method requires rinsing of wafers with deionised water after treatment with a chemical system, followed by drying. The drying process can be time consuming and may also cause particle contamination.

\subsection{Wet Cleaning Chemical Systems}

The different wet cleaning chemical systems used for wafer cleaning include 1) hydrofluoric acid (HF) based solutions, 2) ozone $\left(\mathrm{O}_{3}\right)$ based solutions, 3) sulfuric acid hydrogen peroxide mixtures (SPM), 4) ammonium hydroxide - peroxide mixtures (SC-1), and 5) hydrochloric acid - peroxide mixtures (SC-2). The role of each of these chemical systems in removal of contaminants from wafers is very unique, which has been of great advantage to the wafer cleaning technology.

A common way of removing particles from a surface is by etching. Etching of a surface increases the distance between the particle and the substrate, which in turn lowers the van der Waals forces of adhesion between the two. Hydrofluoric acid (HF) solutions etch the silicon dioxide $\left(\mathrm{SiO}_{2}\right)$ film and therefore have been used in the past to remove 
particulate contamination from silicon wafer surfaces. The etching rate and uniformity of HF solutions depends on the composition and temperature of the solution. Typically, HF solutions are used in the concentrations ranging from $1 \mathrm{HF}: 1 \mathrm{H}_{2} \mathrm{O}$ to $1 \mathrm{HF}: 100 \mathrm{H}_{2} \mathrm{O}$ at $25{ }^{0} \mathrm{C}$ with oxide (undoped thermal oxide) etch rates ranging from $2500 \AA /$ min to $20 \AA /$ min. Etching by $\mathrm{HF}$ leaves the surface terminated with $-\mathrm{SiH}$ or $-\mathrm{SiH}_{2}$ groups. The etching of silicon dioxide by HF occurs according to (R1.1) [1.9-1.12]:

$$
\mathrm{SiO}_{2}+\mathrm{HF}=\mathrm{SiF}_{6}{ }^{2-}+2 \mathrm{H}^{+}+2 \mathrm{H}_{2} \mathrm{O}
$$

Ozone based aqueous solutions have been used in industry for organic contamination removal and photoresist stripping, largely due to their low cost and environmental benefits. Although ozone in the gas phase can oxidize organics and the silicon surface, the rate of oxidation is very low at room temperature [1.13-1.16]. The high oxidation potential $\left(\mathrm{E}^{0}=2.08 \mathrm{~V}\right)$ of ozone in liquid solutions enables its use for removing significant amounts of organic contamination and photoresist [1.17]. However, the application of ozone in aqueous solutions requires a balance between higher solubility of ozone at lower temperatures and increased reaction rates at higher temperatures. There is also a growing interest in the use of ozone - sulfuric acid mixtures due to the higher solubility of ozone in sulfuric acid solutions than in water.

Mixtures of $98 \%$ sulfuric acid and $30 \%$ hydrogen peroxide, known as SPM or Piranha, are used for removal of organic contaminants from the wafer surface due to the 
strong oxidizing power of the solution. The composition of this mixture that is commonly used in industry varies from $4 \mathrm{H}_{2} \mathrm{SO}_{4}: 1 \mathrm{H}_{2} \mathrm{O}_{2}$ to $2 \mathrm{H}_{2} \mathrm{SO}_{4}: 1 \mathrm{H}_{2} \mathrm{O}_{2}$ at temperatures of $100{ }^{0} \mathrm{C}$ or higher. Mixing of sulfuric acid and hydrogen peroxide solution is an exothermic process with final solution temperatures depending on the composition and initial temperature of solution. The powerful oxidants, $\mathrm{HSO}_{5}{ }^{-}$and $\mathrm{H}_{3} \mathrm{O}_{2}{ }^{+}$, formed during the reaction between $\mathrm{H}_{2} \mathrm{O}_{2}$ and $\mathrm{H}_{2} \mathrm{SO}_{4}$ can oxidize the organic impurities on the wafer surface [1.18-1.21].

SC-1 and SC-2 chemical systems, developed by W. Kern in the 1960's at RCA Laboratories, are the most common aqueous based chemical solutions used for removal of organic, particulate and metallic impurities from wafer surfaces [1.22]. SC-1 cleaning involves using mixtures of ammonium hydroxide (29\%), hydrogen peroxide (30\%) and water in the ratios from $0.05 \mathrm{NH}_{4} \mathrm{OH}: 1 \mathrm{H}_{2} \mathrm{O}_{2}: 5 \mathrm{H}_{2} \mathrm{O}$ to $1 \mathrm{NH}_{4} \mathrm{OH}: 1 \mathrm{H}_{2} \mathrm{O}_{2}: 5 \mathrm{H}_{2} \mathrm{O}$ at $70-80{ }^{0} \mathrm{C}$. The $\mathrm{pH}$ of this solution is close to 11 . The use of temperatures higher than $80{ }^{\circ} \mathrm{C}$ is not advised in SC-1 solutions due to the rapid decomposition of peroxide and loss of $\mathrm{NH}_{4} \mathrm{OH}$ as $\mathrm{NH}_{3}$. At the alkaline $\mathrm{pH}$, formation of $\mathrm{HO}_{2}{ }^{-}$species occurs which is strong oxidant with the potential to remove organic contaminants from the wafer surface [1.23-1.24]. This species can also oxidize silicon to silicon dioxide which slowly etches in an alkaline solution. The continuous formation and etching of oxide aids in the removal of particles from the wafer surface. The SC-1 solution is also found to be useful in complexing metals ions such as $\mathrm{Ag}^{+}$and $\mathrm{Cu}^{2+}$ and in the dissolution of metals (Ag, copper, chromium, cobalt, etc.) [1.25]. 
The SC-2 cleaning solution consists of a mixture of hydrochloric acid (37\%), hydrogen peroxide $(30 \%)$ and water in a ratio of $1 \mathrm{HCl}: 1 \mathrm{H}_{2} \mathrm{O}_{2}: 5 \mathrm{H}_{2} \mathrm{O}$ at $70-80{ }^{0} \mathrm{C}$. The $\mathrm{pH}$ of this solution is less than 1 . Metal ions such as $\mathrm{Fe}^{3+}, \mathrm{Al}^{3+}, \mathrm{Zn}^{2+}$ and $\mathrm{Mg}^{2+}$ hydrolyze in the SC-1 solution and form insoluble metal hydroxides that are not removed [1.25]. Hence the SC-2 solution is used since it can dissolve any metal as a metal ion due to the acidic nature of the solution. Although oxidation of silicon is possible in SC-2 due to formation of powerful oxidants $\left(\mathrm{H}_{3} \mathrm{O}_{2}{ }^{+}\right.$etc. $)$from hydrogen peroxide, there is no etching of either silicon or silicon dioxide.

In most cases, the particle removal by these chemistries is carried out by either oxidation or etching of the underlying substrate. The maximum allowed silicon or oxide loss per cleaning step, as dictated by ITRS, is $0.4 \AA$ or less. Under such conditions, use of dilute chemistries or alternative non-reactive chemicals becomes the obvious choice. However, this also puts a restriction on the cleaning efficiencies that can be achieved by employing these chemistries alone. Hence, various external sources of energy are used in conjunction with dilute chemistries for removal of impurities. A typical cleaning sequence of these chemistries used for wafer cleaning is shown in figure 1.5. 


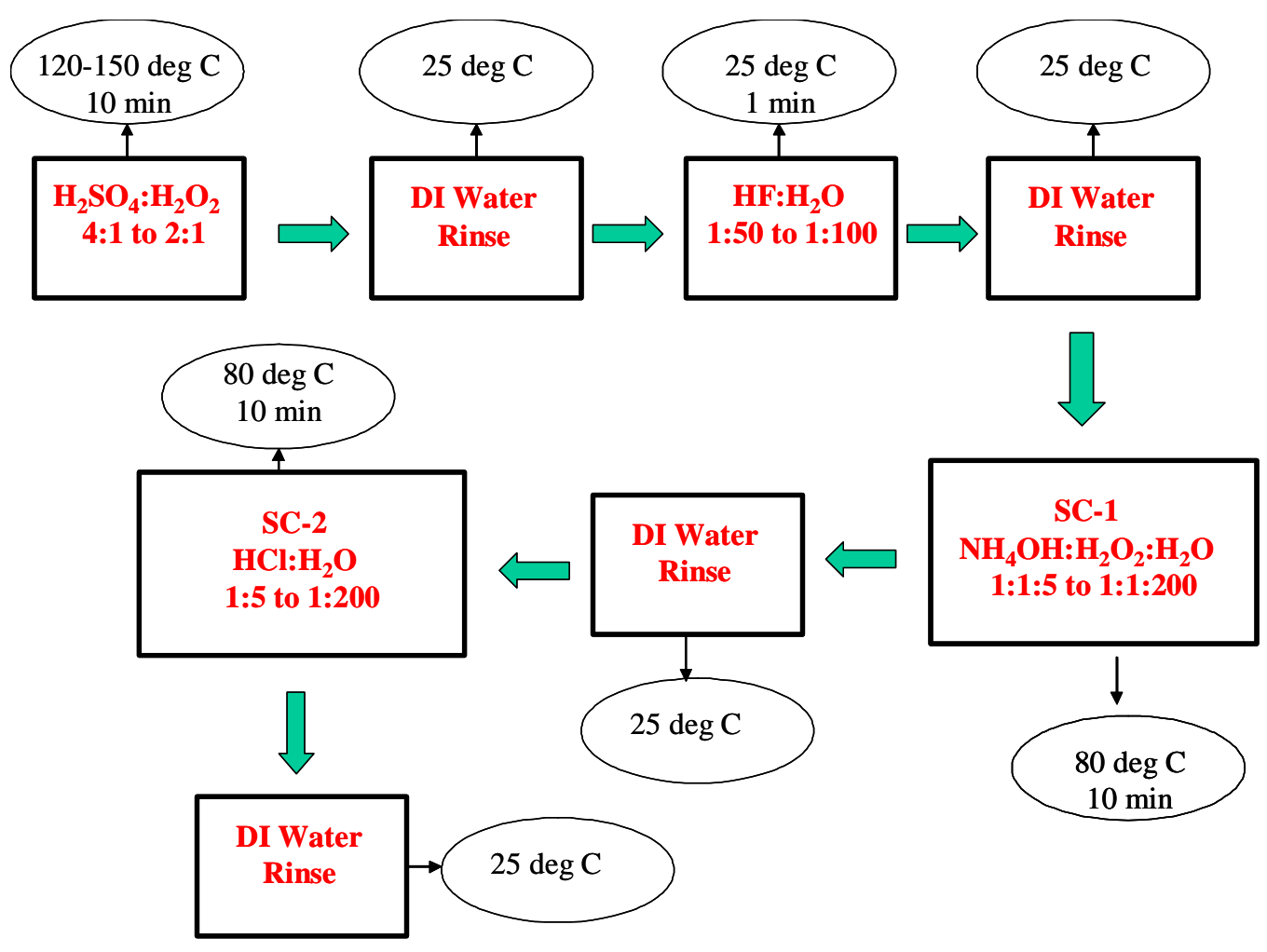

Figure 1.5: Wafer cleaning sequence typically used in semiconductor fabrication [1.25\&1.5]

\subsection{Wafer Cleaning Techniques}

Several wafer cleaning techniques incorporating solution chemistries have evolved over time to meet the contamination challenge. These include 1) immersion cleaning, 2) centrifugal spraying, 3) brush scrubbing, 4) high-pressure fluid jet cleaning and 5) megasonic cleaning. Of these, megasonic cleaning is the most accepted technique in practice today for the removal of particulate contaminants from wafer surfaces. The 
following section discusses some of these widely used cleaning techniques in industry today.

\subsubsection{Immersion Cleaning}

As the name suggests, this technique includes immersion of wafers (or a single wafer) in a sequence in different cleaning solutions contained in separate baths [1.2]. This method of cleaning allows uniform contact of chemical solution on both sides of the wafer [1.27]. In addition, a good control of solution temperature can be obtained using this technique. In a typical wet bench used in industry, the wafer cassette is immersed in a proper cleaning bath maintained at a desired temperature for a specified process time followed by rinsing in DI water in another tank. The wafer may be immersed again in another chemical bath if required and the sequence may then be repeated. This technique, though very useful, requires significant amounts of chemicals and water. Metallic impurities, organic contaminants and soluble material can easily be removed from the wafer surface using strong chemistries but removal of particulate contamination often requires mechanical agitation such as megasonics, bubbling inert gas through the solution etc. [1.9]. These impurities, including some from the wafer cassettes themselves, accumulate in the bath with subsequent cleaning, and hence bath replacement or recirculation using filters is required [Jones, et al., 1987]. In order to reduce contamination due to the wafer cassettes, silicon or carbide carriers are used to reduce the extent of contact with the wafers [1.9]. 
Manual wet benches are being replaced the automatic ones which will reduce contamination and yield better results. A schematic of immersion cleaning is shown in figure 1.6.

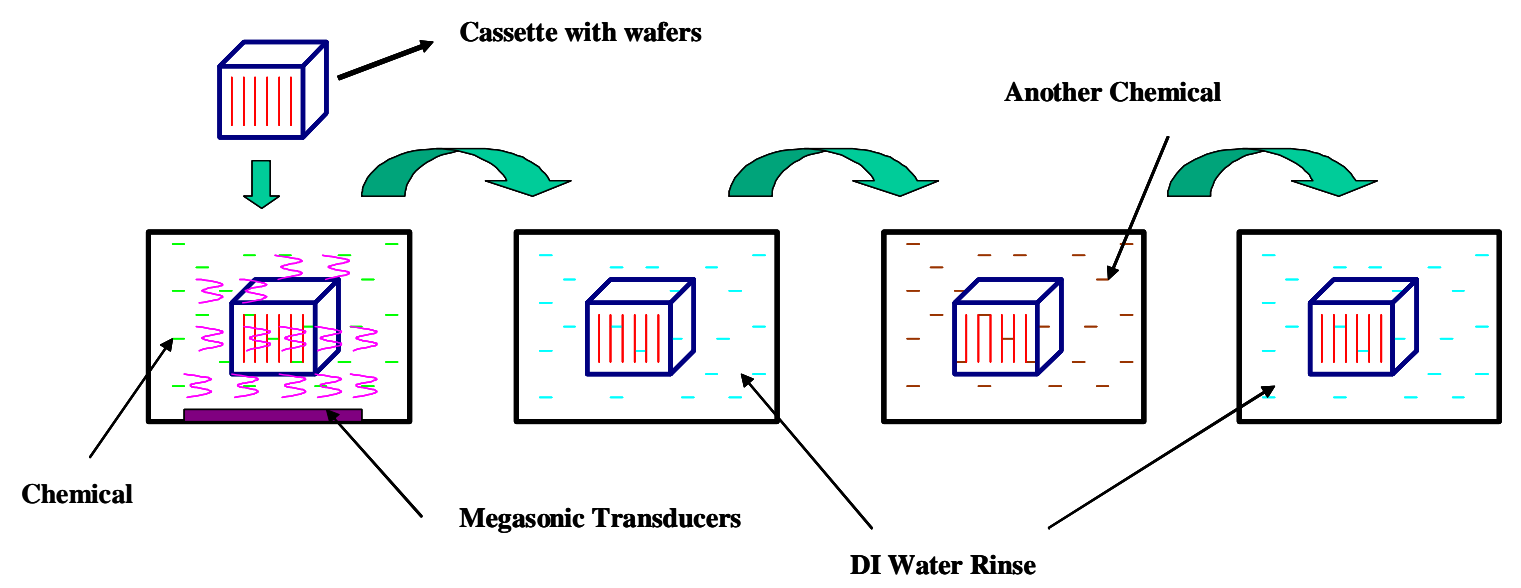

Figure 1.6: Schematic showing immersion cleaning of wafers

\subsubsection{Centrifugal Spray Cleaning}

Centrifugal spray cleaning was first introduced by FSI in 1975 [1.28]. This method involves the use of a spray nozzle to dispose chemicals on the surface of a wafer in a cassette placed on a rotating turntable. When the pressurized liquid spray impinges the wafer surface, its pressure energy is converted to kinetic energy which allows it to change direction and flow parallel to the surface. The fresh chemicals come in contact with the contaminants on the wafer, and used solutions are continuously drained from the bottom of the chamber. The boundary layer thickness depends on the fluid velocity on the 
surface and affects the contact time of the fresh chemical with the impurities on the surface. A better mixing of fluid in the radial direction is obtained by maintaining a turbulent flow of fluid on the surface. The entire cleaning sequence, including rinsing and drying, are performed in the same enclosed chamber which reduces contamination from any external source. In addition, there can be savings in terms of time due to the faster drying by use of centrifugal force. The spray process also eliminates solution contamination and degradation problems associated with immersion techniques due to the drainage of the spent chemical [1.2].

The spray processors have gained importance because of their lower chemical usage, ease of use, space requirements, safer operation and other factors. However, these tools require higher maintenance as they involve movement of parts. A single wafer spray tool illustrating chemical systems, process chamber, rotating chuck, recirculation, and drainage is displayed in figure 1.7. 


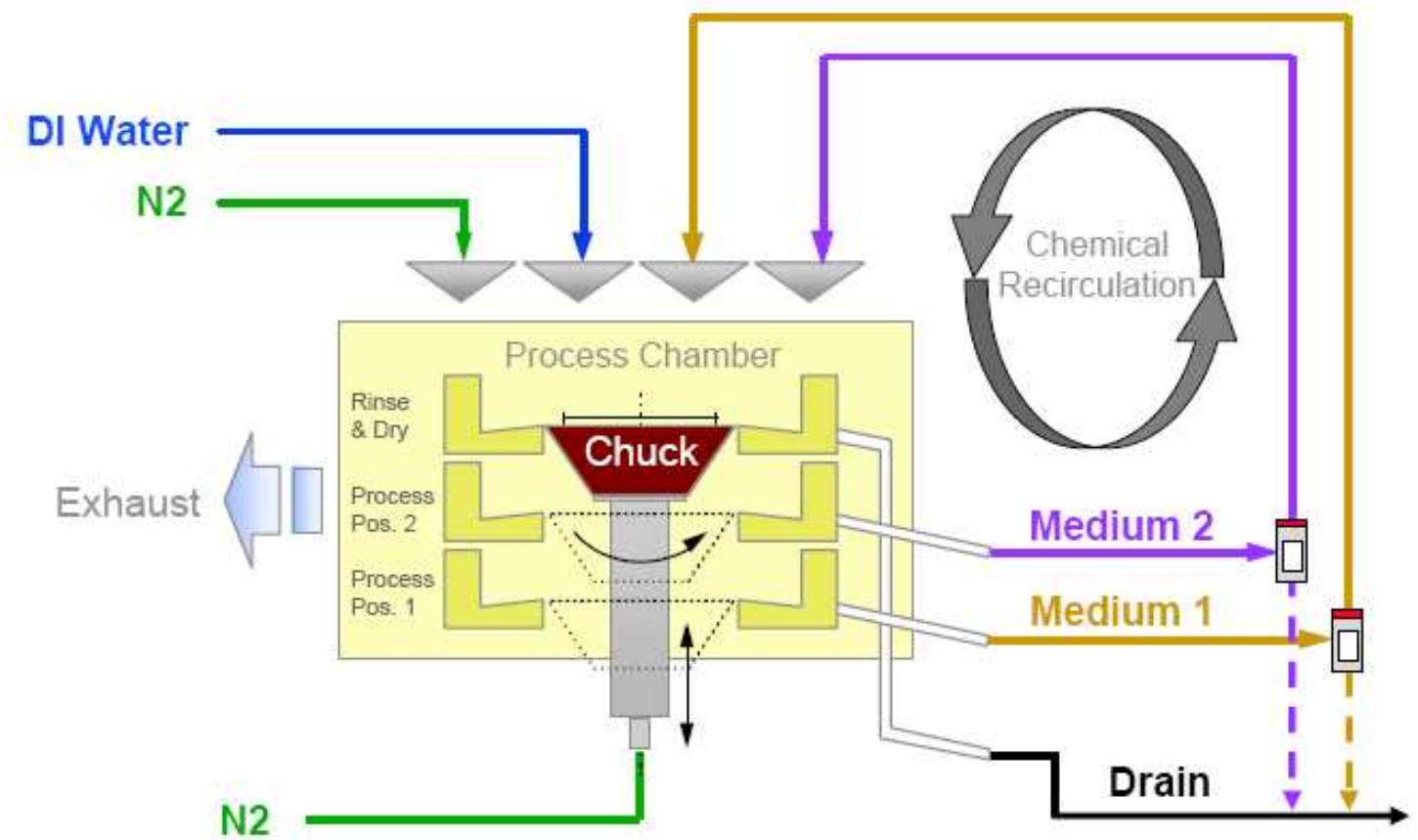

Figure 1.7: Single wafer spray tool (SEZ DaVinci) 


\subsubsection{Brush Scrubbing}

Brush scrubbing is used for hydrodynamic removal of particulate contaminants and organic films from the wafer surface by rotation of a nylon or propylene brush across the surface. In the scrubbing process, the rolling of the brush can generate drag, electrostatic double layer and thermophoretic forces on the particle adhered on the wafer surface [1.29].

The relative motion between the brush and the wafer causes a thin fluid flow which generates a drag on the particle. The thin film, along with the hydrophilic nature of the brush surface, provides a cushioning effect between the surfaces and prevents any scratches or damage to the wafer surface from the bristles of the brush or a large particle. The moment of the drag force can cause rolling of the particle and its removal from the surface. Electrostatic double layer forces arise due to the surface charge and double layer charge interactions [1.30].

In a thermophoretic mechanism, temperature gradients are employed between the wafer and the brush which generate a flow field and force in the direction opposite to that

of the adhesion force between the particle and the wafer [1.31]. A schematic illustrating the forces acting on the particle in brush scrubbing is shown below. 


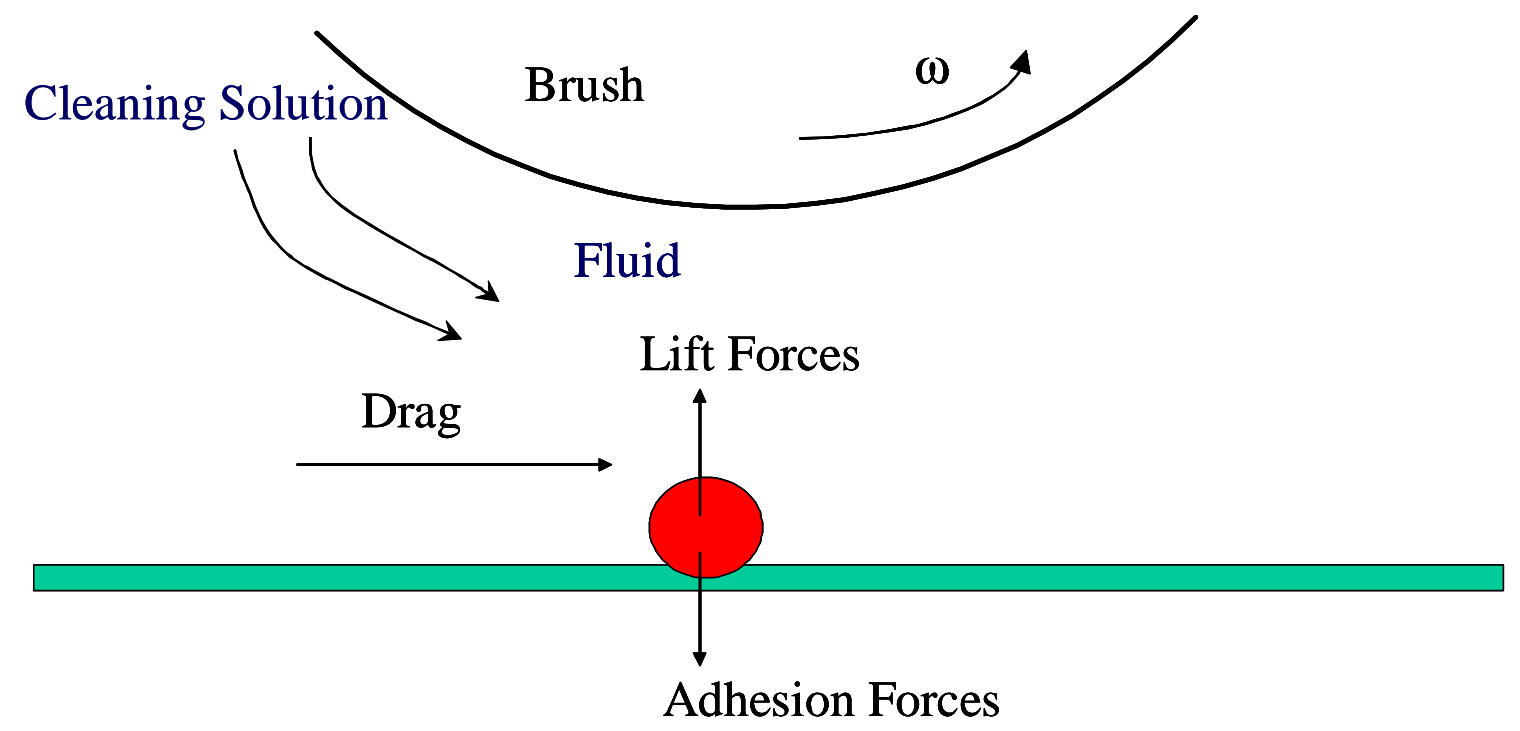

Figure 1.8: Schematic of brush scrubbing process [1.29]

The common chemicals used in brush scrubbing include water, ionic surfactants (e.g. Triton-X), ammonium hydroxide solution, isopropyl alcohol, methanol and others. The organic solvents help to remove organic films and also speed up the drying process. Although brush scrubbing is limited by its capability to remove sub-micrometer size particles, it is still a frequently used technique for post-CMP cleaning [1.32-1.34]. Another limitation of the brush scrubbing technique is that it is unable to remove particles from patterned wafers. This is due to the large size of the bristles which cannot penetrate the thin space between the features. The design of the brush scrubber is very critical and is often considered an art. An improperly designed scrubber can easily get clogged with 
debris and damage the wafer surface. Routine maintenance and replacement of scrubbers is another requirement associated with brush scrubbing.

\subsubsection{High Pressure Fluid Jet Cleaning}

High pressure fluid jet cleaning has been in use for many years for removing particulate contaminants from surfaces. It consists of a high velocity fluid jet that sweeps over a surface at pressures of approximately 4000 psi [1.35-1.36]. Due to higher densities of liquids compared to gases, they have an advantage over gases in removing smaller particles and hence are more effective for cleaning of surfaces. In addition, static charge build up is another problem often associated with the use of gases.

The different chemicals that can be used in jet cleaning include DI water, chemistries employing $\mathrm{HF}, \mathrm{HCl}, \mathrm{NH}_{4} \mathrm{OH}, \mathrm{H}_{2} \mathrm{O}_{2}$ etc, ozonated water and organic solvents. The boundary layer thickness of these liquids on the surface is controlled by the rotation speed of the wafer. In some cases a mixture of liquids and gases may also be used [1.37]. A GasJet technique has separate nozzles for liquids and gases which are concurrently delivered on the wafer surface. Oscillating forces for removal of particles can be generated in such systems even in the absence of a sonic source [1.38]. Carbon dioxide gas in dissolved form is used to reduce water resistivity in order to avoid the problem of static electricity in high pressure scrubbing. 
A proper design of liquid pressures and velocities is essential to prevent any damage to the surface. The nozzle design features that need to be optimized to minimize any momentum loss of the fluid are sweep distance and speed, angle of incidence, uniform wafer coverage, and nozzle size for optimum fluid velocity and pressure. The high velocity liquid penetrates the trenches in the patterned wafers and removes any clogged debris by transfer of momentum to the particles. This technique has gained importance over brush scrubbing due to its non contact mode for cleaning. A schematic of high pressure fluid jet cleaning is shown in figure 1.9.

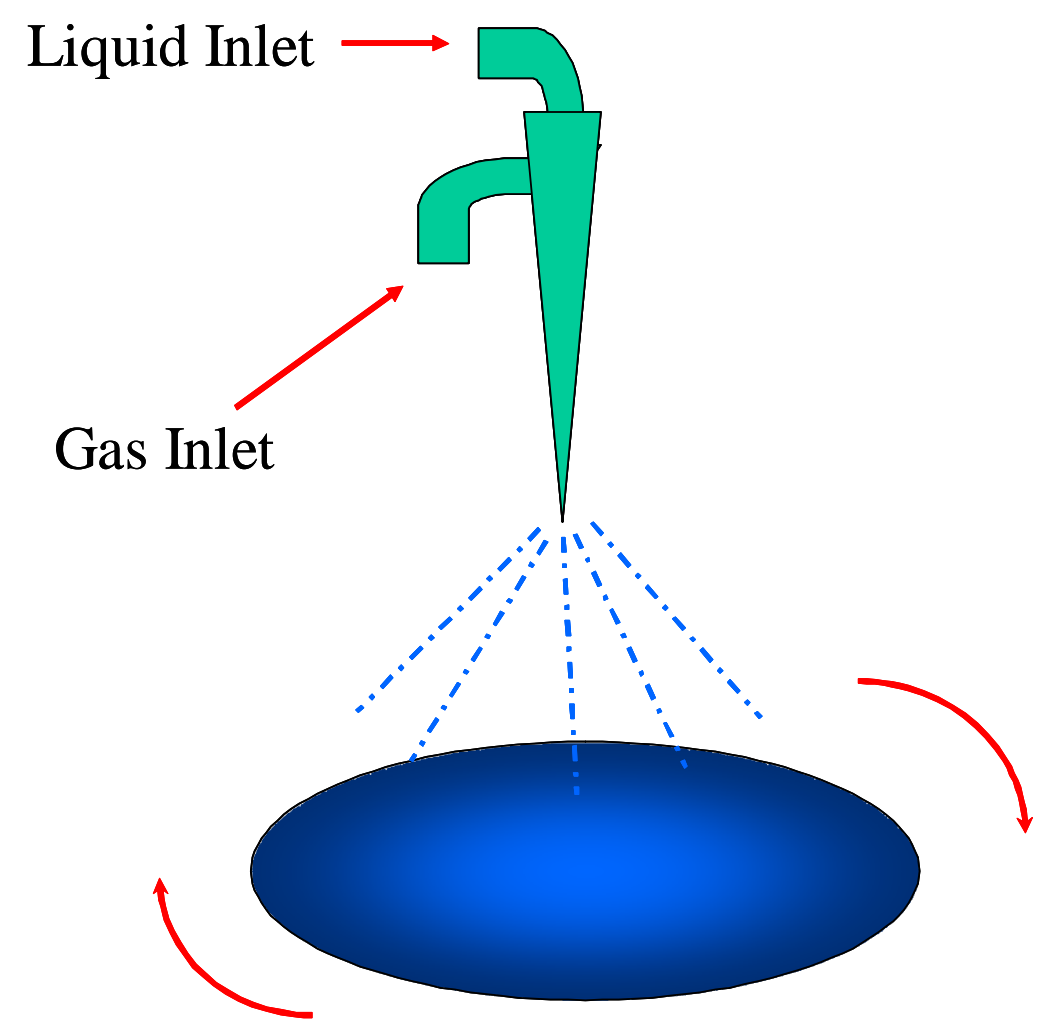

Figure 1.9: Schematic of high pressure fluid jet cleaning system 


\subsubsection{Megasonic Cleaning}

Megasonic cleaning is routinely used in the semiconductor industry to remove small particles from wafer and mask surfaces. Cleaning is achieved through proper choice of chemical solutions, power density and frequency of the acoustic field. Two principal mechanisms, namely acoustic streaming and acoustic cavitation, are considered to be responsible for the removal of particles from a contaminated surface.

Acoustic streaming refers to time independent fluid motion due to the loss of acoustic momentum caused by viscous attenuation and wave interactions with solid boundaries. Particle removal in megasonic cleaning relies on reduction in boundary layer thickness at the solid-fluid interface that is achieved by means of three types of streaming. Eckart streaming, which occurs outside the boundary layer, is characterized by vortices on the scale of the flow field [1.39]. It reduces the diffusion boundary layer thickness and thus increases the chemical reactivity at the surface. Schlichting streaming (boundary layer streaming) results from attenuation due to continuity of the acoustic displacement field at the solid - viscous fluid interface [1.7]. It is characterized by vortices much smaller than the acoustic wavelength. The acoustic streaming velocity is proportional to the product of the square of the acoustic frequency and transducer intensity and inversely proportional to viscosity. In a megasonic field with a transducer intensity of $10 \mathrm{~W} / \mathrm{cm}^{2}$, the streaming velocity can be as high as $5.4 \mathrm{~m} / \mathrm{sec}$ at $0.925 \mathrm{MHz}$ in water. Thus, these two types of streaming can cause high viscous stresses and large velocity gradients in the 
boundary layer for the removal of contaminants from the substrate. In addition, the acoustic boundary layer thickness $(\delta)$, in a fluid with a kinematic viscosity of $v$, decreases with an increase in the angular acoustic frequency $(w)$.

$$
\delta=\left(\frac{2 v}{w}\right)^{1 / 2}
$$

At $1 \mathrm{MHz}$, the acoustic boundary layer thickness in water is about $0.5 \mu \mathrm{m}$. By comparison, the turbulent hydrodynamic boundary layer for a free stream velocity of 10 $\mathrm{m} / \mathrm{s}$ on a flat surface is greater than $1600 \mu \mathrm{m}$ for water. Thus, a particle in an acoustic field is subjected to much higher fluid velocity than would occur in a hydrodynamic flow for the same free stream velocity of the fluid.

In the case of acoustic cavitation, stable and transient cavities form when the fluid is subjected to an oscillating pressure field [1.40]. Stable cavitation, acting as a secondary sound source and leading to microstreaming, entails only small oscillations of bubbles about an equilibrium size, while transient cavitation is characterized by large bubble size variations and eventual bubble collapse, which can be quite violent. This violent collapse, accompanied by extremely high temperature and pressure conditions, often leads to the formation of shock waves. Both microstreaming and shock waves can be instrumental in dislodging particles from the wafer surface. Figure 1.10 illustrates the different particle removal mechanisms that are present during megasonic cleaning. 
In the case of cleaning of patterned wafers, the megasonic field provides an oscillating acoustic pressure that is known to enhance mass diffusion and convection in the trench. High power densities, however, limit the use of megasonic cleaning in patterned wafers due to the significant level of damage of the pattered structures [1.42].

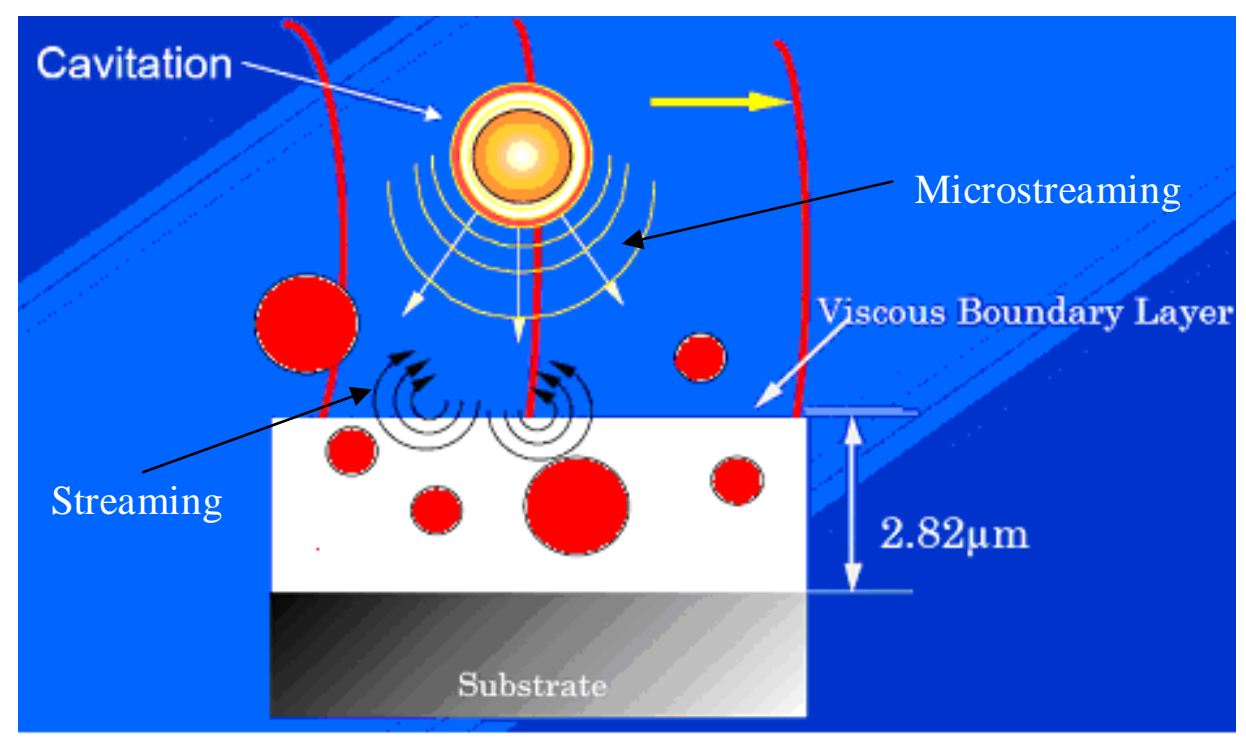

Figure 1.10: Schematic showing particle removal mechanisms in megasonic cleaning [1.41]

Considerable work has been done to understand the particle removal mechanisms in megasonic cleaning using different solution chemistries. Bakhtari, et al., studied the removal of polystyrene latex (PSL) nano-particles from silicon wafers in SC1 and DI water solutions at $0.760 \mathrm{MHz}$ [1.43]. The proposed cleaning mechanism was based on acoustic streaming for dislodging the particles and double layer repulsion force for preventing re-deposition of the particles. Lifting, sliding and rolling of the particles 
resulting from a higher ratio of drag force moment to adhesion force moment has been shown to be useful in megasonic cleaning of substrates [1.44]. Gale, et al., concluded that the particles get removed from the wafer surface due to microstreaming and stable cavitation but are transferred to the bulk solution away from the wafer by means of acoustic streaming [8].

In another megasonic cleaning study by Busnaina, et al., the removal of PSL and silica particles of different sizes was investigated at $0.862 \mathrm{MHz}$ at $150 \mathrm{~W}$ in $\mathrm{SC} 1$ $\left(1 \mathrm{NH}_{4} \mathrm{OH}: 1 \mathrm{H}_{2} \mathrm{O}_{2}: 5 \mathrm{DI}\right)$ solution as a function of time. The cleaning time for complete removal of particles smaller than $300 \mathrm{~nm}$ from the wafer surface was found to be $20 \mathrm{~min}$ or greater [1.45]. Such high cleaning time can cause excessive loss of wafer surface due to continuous oxidation and etching in SC1 solutions. The use of DI water instead of SC1 eliminates the problem of silicon/oxide loss but requires much higher transducer intensity to achieve a comparable level of cleaning.

Shende investigated the effect of transducer intensity on damage of silicon on oxide structures in DI water [1.46]. He found that even at a power density of $2.17 \mathrm{~W} / \mathrm{cm}^{2}$, a significant level of damage to features is possible. Figure 1.11 shows a typical breakage of test structures (single crystalline silicon fins on oxide surface) during megasonic cleaning. Thus, there is a trade-off between complete particle removal and damage from megasonic cleaning. To meet the particle challenge, it is necessary to introduce nonetching chemistry with advanced non-damaging megasonics. In the present study, the use 
of simple electrolytes in megasonic cleaning has shown potential for efficient and damage free cleaning of wafers and other surfaces used in the semiconductor industry. A cleaning mechanism, based on electro-acoustic effects, has been identified that allows particle removal at much lower megasonic power densities with negligible surface etching.

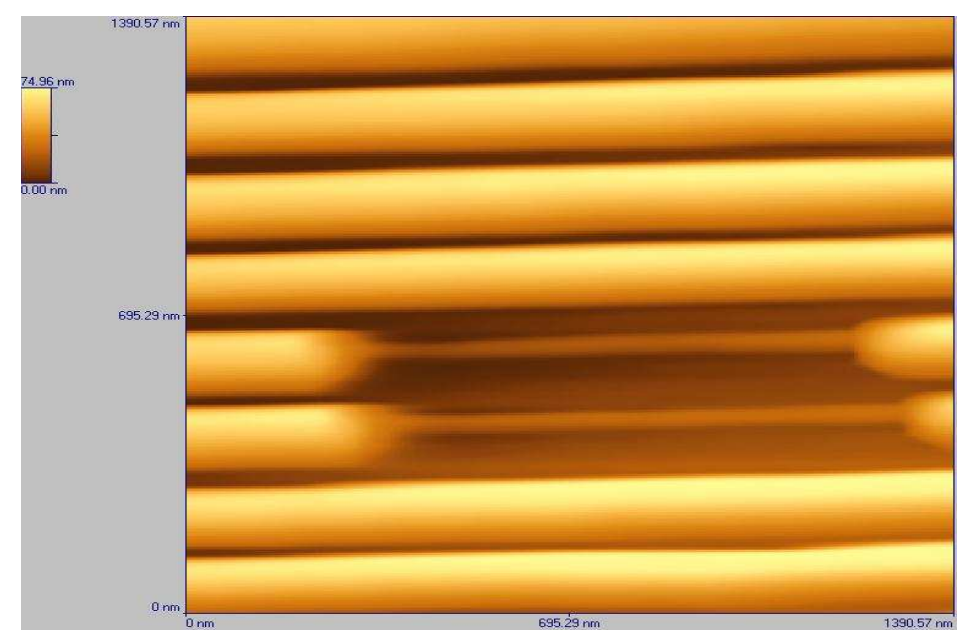

Figure 1.11: Damage of test structures (silicon fins on oxide surface) during megasonic cleaning (Courtesy of Prof. Raghavan) 


\subsection{Research Objectives}

The overall objective of this work is to investigate the role of electrolytes in removal of charged particles from wafer surfaces in megasonic cleaning. The specific objectives are as follows:

1. Determine experimentally the effect of different electrolytes on removal of silica particles from silicon wafers in megasonic cleaning. The variables considered in the study include type of electrolyte, surface charge on silica particles, transducer power density, and solution parameters such as ionic strength, temperature, $\mathrm{pH}$ and cleaning time. Examine the role of ionic vibration potential (IVP) and colloid vibration potential (CVP) in megasonic cleaning.

2. Evaluate the importance of solution properties such as bulk modulus and density in modulating the pressure amplitude of the sound wave. Perform hydrophone studies to determine the pressure amplitude in electrolyte solutions of different concentration and its impact on stable bubble size distribution.

3. Characterize the cavitation process in electrolyte solutions using sonoluminescence measurements. 


\section{CHAPTER 2: MEGASONICS}

\subsection{Introduction}

In present day devices, the dimensions of the smallest structures are close to that of the particles that need to be removed from wafer surfaces. The importance of cleaning processes that remove nano-particles between process steps without damaging the fragile devices is continuously growing. The restrictions on material loss during cleans prevents the use of aggressive chemicals. As an alternative to pure chemical cleaning, dilute chemistries are used in combination with physical forces to drag the small particles on the surface [2.1]. One such means of applying physical force is megasonic cleaning; a noncontact mode of cleaning that removes sub-micron particles from both sides of the wafer. This technique was first patented by Stanley Shwartzman and Alfred Mayer of RCA Solid State Technology Center [2.2].

In megasonic cleaning, sonic waves with a frequency of approximately $1 \mathrm{MHz}$ are directed from the transducer array panels either parallel or perpendicular to the wafers that are immersed in the media liquid. The transducers are made up of piezoelectric materials that possess the ability to convert electrical energy into mechanical energy when high-frequency AC voltage, between 500 and $2000 \mathrm{kHz}$, is applied causing the transducer material to rapidly change dimension or vibrate. The resonant masses of the 
transducer transmit these vibrations into the liquid, producing acoustic waves in the cleaning fluid [2.3].

Megasonic cleaning can be used either for single wafer or batch wafer processing. The major advantages of single wafer processing include increased flexibility and shorter cleaning times. In addition, the risk of wafer scrap is significantly reduced by processing wafers individually [2.4]. In a typical megasonic cleaning process in industry, wafers are randomly moved across the transducers to cover the entire acoustic field so that uniform and better cleaning can be achieved. In some cases, the acoustic field is spatially moved by temporal variation of voltage to various transducers in the cleaning tank. Development of 'cold spots' or areas of ineffective cleaning is commonly seen on wafers when one of the transducers loses its intensity or the wafers fail to scan across the entire acoustic field. The measurements of variation in transducer intensity in the cleaning tank are necessary to avoid non-uniform cleaning of the wafers and to improve cleaning efficiency. An example of such measurements is shown in figure 2.1 where cavitation intensity was measured spatially across the three transducers arranged side by side in the cleaning tank [2.5].

The zones of lowest transducer intensity indicated the regions between the transducers where lowest cavitation occurred. This suggested that one can expect regions of reduced intensity during megasonic cleaning that will impact the contamination level on the wafers. 


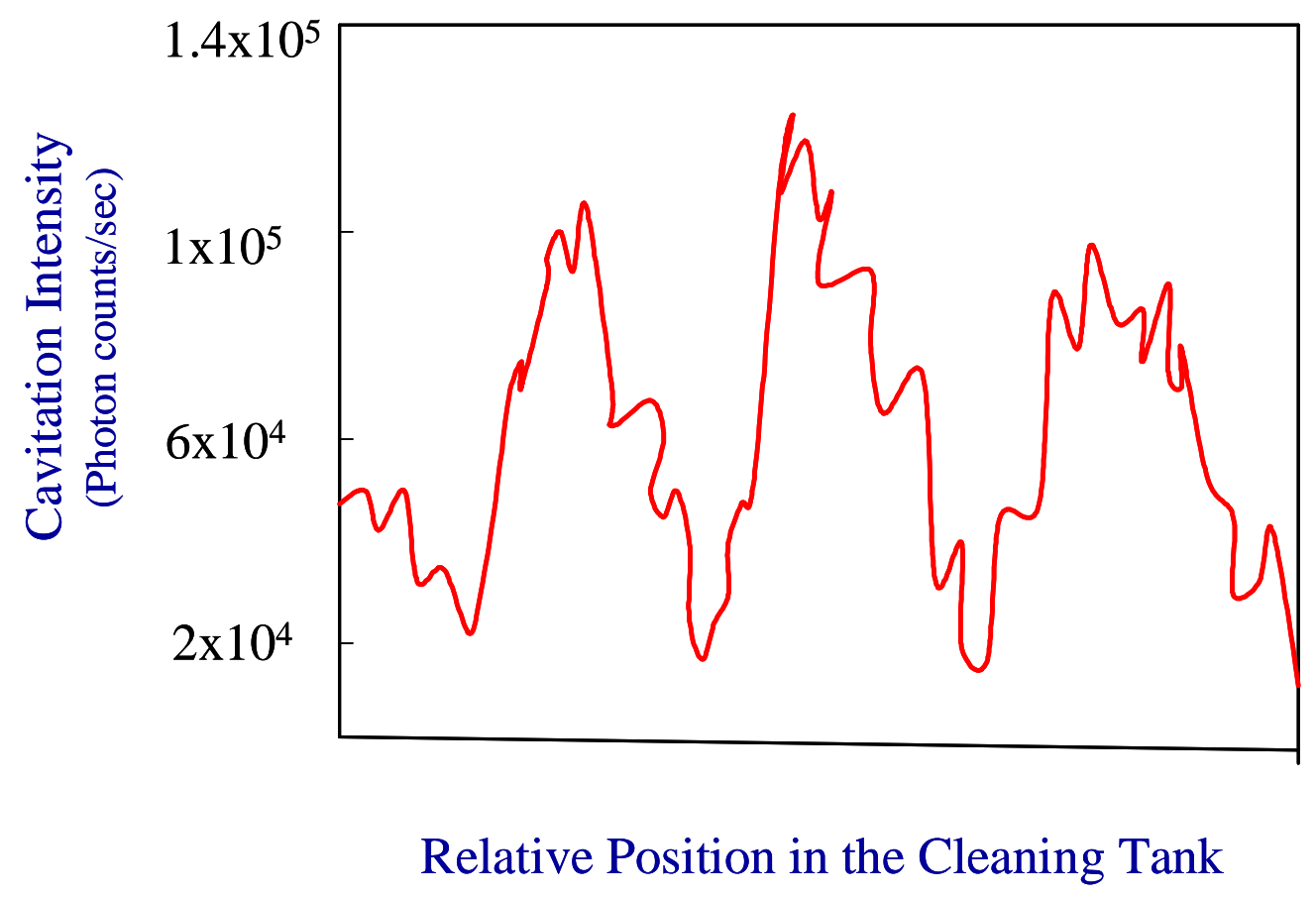

Figure 2.1: Spatial variation in cavitation in the megasonic cleaning tank [2.5]

The factors that affect megasonic processing include sound frequency, transducer intensity, chemistry, process time and temperature. The most important physical parameters, however, are turning out to be the bubble cavitation, acoustic and viscous boundary layer and acoustic streaming induced forces. A schematic showing these mechanisms in an immersion type megasonic cleaner is represented in figure 2.2 


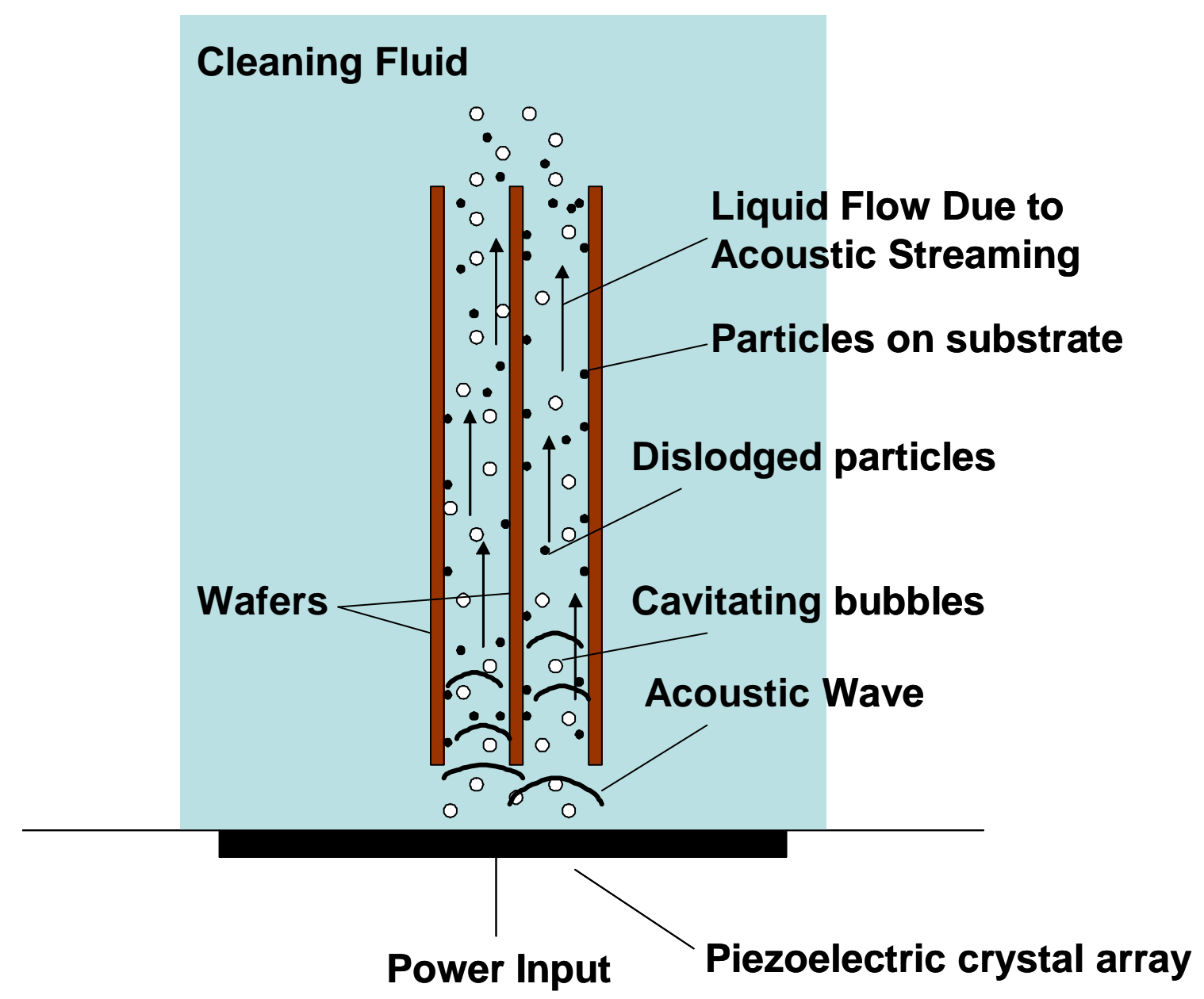

Figure 2.2: Schematic showing cleaning mechanisms in an immersion type megasonic cleaner [2.3] 


\subsection{Cleaning Mechanisms}

Although several mechanisms have been known to exist in megasonic cleaning of surfaces, the actual cleaning mechanism has been a topic of debate for many years. Olim estimated that the acoustic pressure force, which acts parallel to the surface due to the pressure gradient in the direction of sound wave propagation, cannot remove particles smaller than $350 \mathrm{~nm}$. The removal of these particles in megasonic cleaning requires additional cleaning mechanisms [2.6].

Farrell, et al., discuss two mechanisms by which wafer cleaning can be achieved, one by direct interaction of the sound wave with the particle adhered to the wafer and the other due to cavitation [2.5]. It was suggested that the propagation of the sound wave generates a periodic movement of the fluid element which will cause displacement of the particle. If the particle displaces sufficiently away from the surface, it may get detached. However, simple calculations show that the fluid element in water at $1 \mathrm{MHz}$ of sound frequency displaces only a few angstroms from its equilibrium position at a transducer power density of $1 \mathrm{~W} / \mathrm{cm}^{2}$ and is unlikely to be the particle removal mechanism (Kinsler and Frey 1962).

Shwartzman, et al., claimed that the time for cavity formation in megasonic cleaning is too short $(1.25 \mu \mathrm{s})$ and that rocking action due to sound waves, rather than cavitation, is the primary cleaning mechanism [2.2]. Deymier, et al., showed that the 
acoustic pressure force due to the scattering of acoustic wave by a particle adhered on the wafer surface is negligible and therefore unlikely to generate a significant pressure field to cause removal of particle from the surface [2.7]. However, after numerous discrepancies, two major cleaning mechanisms have evolved to become widely accepted mechanisms for megasonic removal of particles. These include acoustic streaming and cavitation.

McQueen considered the usefulness of acoustic streaming in reducing the boundary layer thickness based on her work on removal of nano-particles from surfaces [2.8-2.9]. Deymier et al. investigated the effect of second order sound fields such as Schlichting streaming on removal of particles in megasonic cleaning [2.10]. They treated the solid as an isotropic elastic medium and water as a viscous fluid. It was shown that the normal component (perpendicular to the wafer surface) of the removal force resulting from the second order acoustic field was too small to remove the submicron sized particles. However, they predicted that the parallel component of the streaming force can exert significant drag on the particle and remove it through a rolling mechanism.

Gale, et al., explained the role of cavitation and acoustic streaming in megasonic cleaning [1.39]. They suggested that stable cavitation results in strong microstreaming currents close to the wafer surface that can induce cleaning. Acoustic microstreaming occurs due to the large stable oscillations of the gaseous cavities which in turn causes the rapid movement of the surrounding liquid in the same pattern as the bubble wall. These 
gaseous cavities can either nucleate on a solid surface or pre-exist in the liquid solution. If asymmetry in the bubble oscillations occurs, say due to the presence of a boundary of a particle or a surface, intense microstreaming patterns develop causing significant shear stresses along the boundary.

In addition to stable cavitation, shock waves and liquid jet formation occurs by cavity collapse causing particle detachment. The detached particle is then carried away from the wafer by streaming forces, due to strong currents and boundary layer thinning. The possible role of surface tension effects due to bubble oscillations in removal of particle from the wafer surface has been also considered [1.39].

Microjet formation is often observed in degassed liquids where inertially controlled oscillating bubbles with large displacement amplitudes become unstable following an asymmetry in the flow field around them. This causes one wall of the bubble to collapse faster than the other, thereby forming a liquid jet that can attain supersonic velocity and impact the boundary that caused asymmetry in the first place. If the boundary happens to be a particle surface, it can get dislodged from the wafer as a result of this impact. This form of cavitation is believed to be the primary mechanism for removal of particulate contaminants from surfaces during cleaning in a megasonic field. When the same effect is present in ultrasonic cleaning of wafers, damage of the surface can occur in addition to removal of particles. 
Busnaina, et al., considered three different mechanisms that may contribute to particle removal: lifting, sliding, or rolling [1.44]. Zhang, et al., proposed that particles will be removed if the drag force $\left(F_{D}\right)$, lift force $\left(F_{L}\right)$ and the adhesion force $\left(F_{a}\right)$ satisfy the following condition with $C_{F}$ being the coefficient of friction [2.11]:

$F_{D} \geq C_{F}\left(F_{a}-F_{L}\right)$

The drag force in the above equation is given by equation 1.3. In a megasonic field, the lift force is normally very small and can be neglected compared to the adhesion force [1.46]. The removal of a particle by rolling can occur when the ratio of the hydrodynamic rolling moment to the adhesion resisting moment, $R M$, is much larger than 1 [2.12].The rolling mechanism with different parameters used in equation 2.2 is shown in figure 2.3.

$$
R M=\frac{F_{D}(1.399 R-\delta)}{F_{a} a}
$$




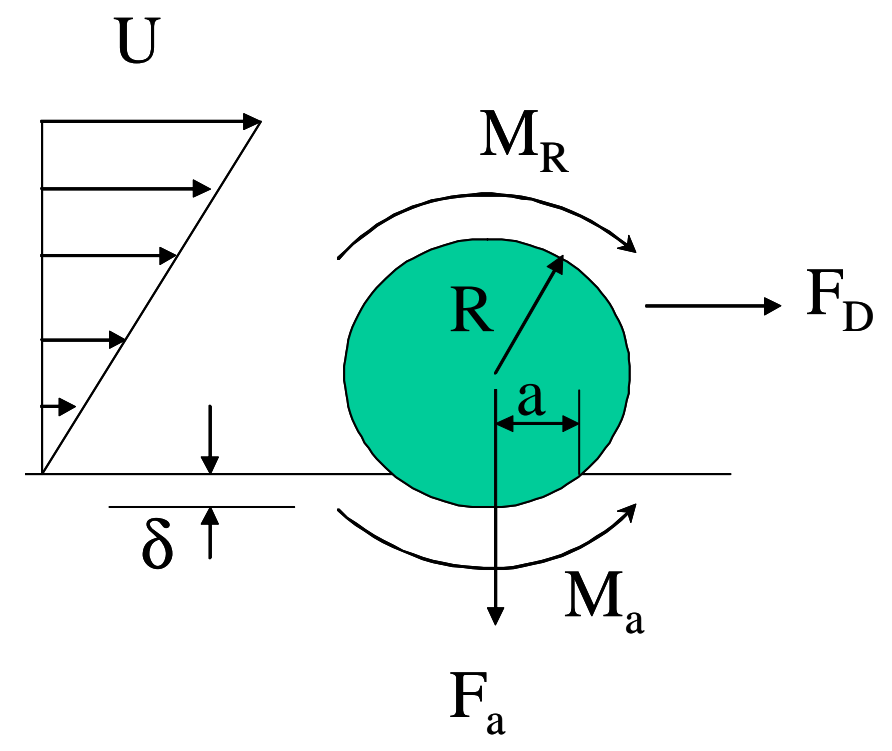

Figure 2.3: The different forces involved in a rolling removal mechanism [1.44]

\subsection{Forces on a Particle in a Megasonic Field}

A particle on a wafer, immersed in a liquid, experiences several types of forces acting on it. The most important of these forces include van der Waals adhesion forces and double layer interactions (electrostatic forces of interaction). When a megasonic field is applied, additional drag forces (described in section 2.2) due to acoustic streaming or cavitation are possible. These forces have been used traditionally to explain the removal of particles from wafer surfaces. In this work, we propose the existence of two new force components that appear when acoustic waves are passed through a colloidal dispersion containing ions. These forces are based on electro-acoustic effects and have shown higher particle removal capability than other drag forces that have been known to exist during 
megasonic cleaning of wafers. The following section gives a brief discussion of these forces.

\subsubsection{Van der Waals Forces}

Van der Waals forces are one of the most important forces in the adhesion of particles to any substrate. These forces originate due to the instantaneous dipole interactions between molecules formed due to the positions of the electrons surrounding the nuclei. There are three types of dipole interactions, namely permanent dipolepermanent dipole (Keesom interactions), permanent dipole-induced dipole (Debye interactions) and induced dipole-induced dipole interactions which collectively contribute to van der Walls forces [2.13]. The van der Waals forces depend on the composition of the interacting particle, substrate and the medium, the distance of separation between them, and the surface roughness and geometry of the particle and the substrate [2.14]. When the microscopic dipole interactions are summed over all the molecules in a macroscopic body, van der Waals forces between different geometries can be obtained. For example, the van der Waals forces between a spherical particle of radius $\mathrm{R}$ and a flat surface is given by equation 2.3 (Ranade 1987).

$F_{v d}=\frac{A_{132} R}{6 Z^{2}}$ 
where $R$ is the radius of the particle, $A_{132}$ is the Hamaker constant for particle 1 and substrate 2 with medium 3 in between and $Z$ is the distance of separation between the particle and the substrate.

The potential energy of interaction, obtained by integrating the equation 2.3 over the distance of separation, is given as:

$$
E_{v d}=-\frac{A_{132} R}{6 Z}
$$

The Hamaker constant $A_{132}$ can be evaluated using the equation:

$$
A_{132}=A_{33}+A_{21}-A_{32}-A_{31}
$$

where $A_{\mathrm{ij}}$ is the Hamaker constant for two particles in vacuum.

A geometrical mixing rule relates the Hamaker constants between similar and dissimilar particles. Table 2.1 presents Hamaker constants for some materials of practical interest

$$
A_{i j}=\left(A_{i i} A_{j j}\right)^{1 / 2}
$$


Table 2.1: Hamaker constants for different materials [2.13, 2.15, \& 2.16]

\begin{tabular}{|l|l|}
\hline Materials & Hamaker Constant (Joules) in vacuum $\left(\mathrm{A} \times 10^{20} \mathrm{~J}\right)$ \\
\hline Alumina & 15.4 \\
\hline Gold & 45.3 \\
\hline Silver & 39.8 \\
\hline Polystyrene & $7.8-9.8$ \\
\hline Metals & $16-45$ \\
\hline $\mathrm{Si}_{3} \mathrm{~N}_{4}$ & 16.7 \\
\hline$\beta-\mathrm{Si}_{3} \mathrm{~N}_{4}$ & 18 \\
\hline $\mathrm{SiO}_{2}$ & $5.5-6.3$ \\
\hline TiO & \\
\hline KCl & 15.3 \\
\hline NaCl & 5.5 \\
\hline CsI & 6.5 \\
\hline Toluene & 8.0 \\
\hline Acetone & 5.4 \\
\hline Water & 4.2 \\
\hline
\end{tabular}

The potential energy of interaction between pairs of solid surfaces of different geometries is shown in figure 2.4. 


\section{Two spheres}
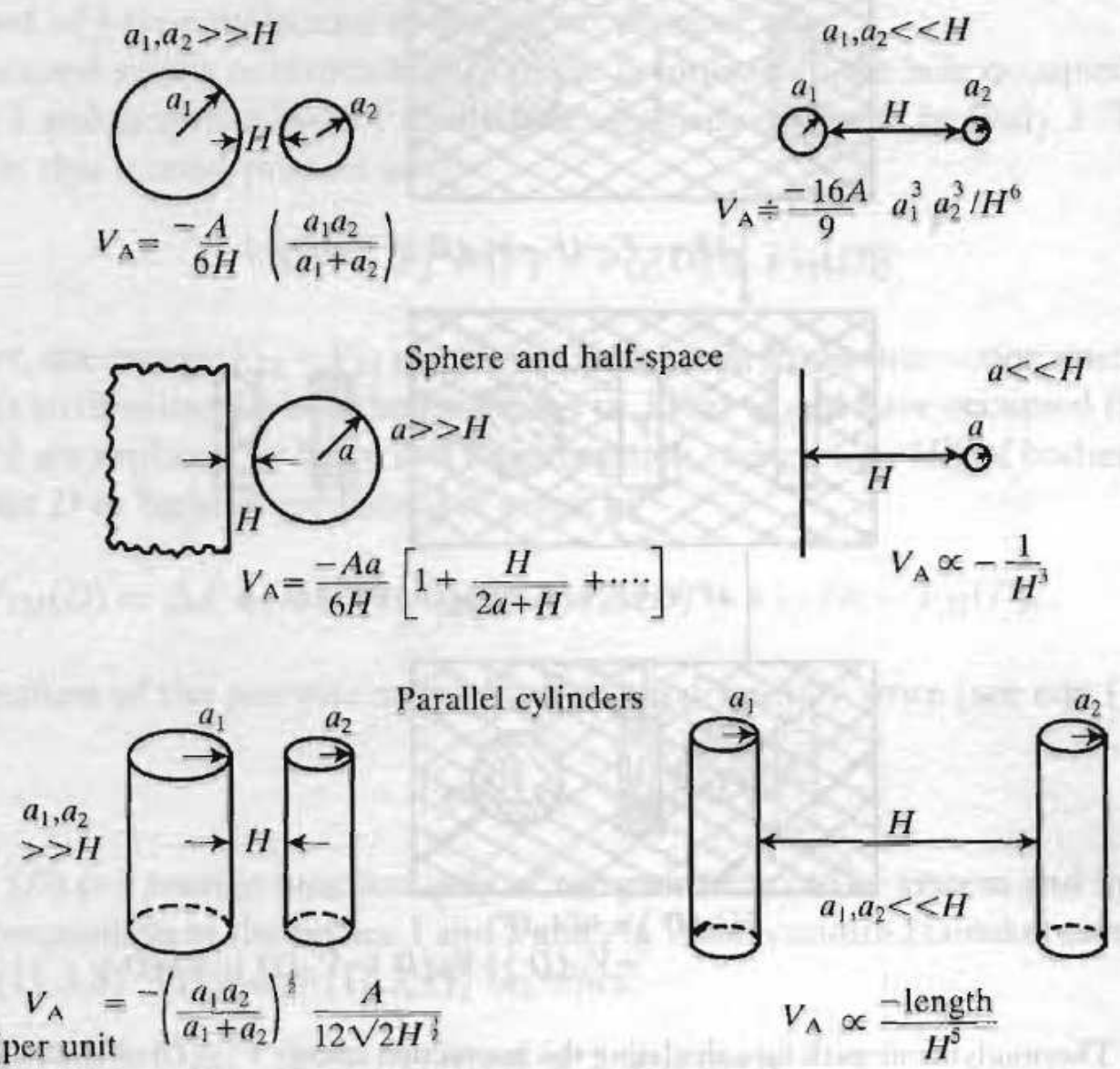
length)

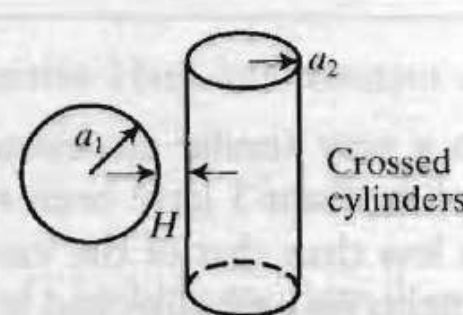

$$
V_{\mathrm{A}}=-\left(a_{1} a_{2}\right)^{\frac{1}{2}} \mathrm{~A} / 6 H
$$

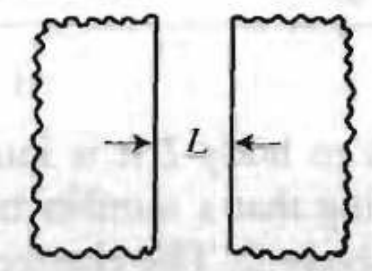

$V_{\mathrm{A}}$

(per unit $=-A / 12 \pi L^{2}$ area)

Figure 2.4: Non-retarded potential energy between pairs of solid surfaces of various geometries [2.17] 
In the case of soft particles, especially when they are aged on the wafer surface for sufficient time, deformation can occur which can increase the contact area between the particle and the surface, leading to enhanced adhesion forces. Depending on the nature of deformation, such as elastic or plastic, different theories including Johnson-KendallRoberts (JKR), Derjaguin-Mueller-Toporov (DMT), and Maugis-Pollock (MP) have been proposed for computing the contact area between the particle and the surface [2.18-2.20]. Kumar et al. have shown that the van der Waals force for a deformed smooth particle and a substrate can be calculated using equation 2.7 [2.14].

$$
F_{v d}=\frac{A_{132} d}{12 h^{2}}\left[1+\frac{2 a^{2}}{h d}\right]
$$

where $h$ is the distance of separation between the particle and the surface, $a$ is the contact radius, $d$ is the diameter of the particle

When surface roughness is taken into account, a Rumpf equation [2.21, 2.22] can be used which considers the asperities to be hemispherical on the substrate.

$$
F_{v d}=\frac{A_{132} R}{6 a^{2}}\left[\frac{1}{1+\frac{R}{r}}+\frac{1}{\left(1+\frac{r}{a}\right)^{2}}\right]
$$


where $R$ is the radius of the particle, $r$ is the radius of the asperity, and $a$ is the distance of separation between the particle and the asperity.

In the case of non-hemispherical asperities, which exist in most practical applications, the van der Waals forces can be calculated using the root mean square (rms) roughness, $b$, and the peak to peak distance, $\lambda$, obtained using atomic force microscopy (AFM) measurements.

$F v d=\frac{A_{132} R}{6 a^{2}}\left[\frac{1}{1+\frac{R}{r}}+\frac{1}{\left(1+\frac{z_{\max }}{a}\right)^{2}}\right]$

where $r=\frac{\lambda^{2}}{58.14 b}$ and $z_{\max }=1.817 b$

\subsubsection{The Double-Layer Interactions}

When a substrate is immersed in a solution containing ions, it can acquire charges either by adsorption of specific ions or by dissociation. The potential of the charged surface depends on the activity of the potential determining ions and is given by the Nernst equation shown below. 
$\Psi_{0}=\frac{2.303 R T}{F} \log \left(\frac{a}{a_{I E P}}\right)$

where $\Psi_{0}$ is the potential difference between the charged surface and the bulk solution, $a$ and $a_{I E P}$ are the activities of the potential determining ions at the solution condition and isoelectric point respectively , $R$ is the gas constant, $T$ is the temperature of the system, and $F$ is the Faraday's constant

The charging of a surface affects the distribution of ions surrounding it, resulting in an increased concentration of counter ions and a decreased concentration of co-ions. The layer of liquid in immediate contact with the solid surface is strongly bound and is referred to as the stern layer. The ions surrounding the stern layer are mobile and the concentration profile of ions in the region is a result of a balance between the thermal energy of the ions and the electrostatic interaction between the ions and the charged surface. This region is referred to as the diffuse layer. The stern layer and the diffuse layer collectively form a double layer. A shear plane exists in the double layer that is described as an imaginary sphere around a particle inside which the solvent moves with the particle as the particles move through the solution. The potential at the shear plane is known as the zeta potential (ZP). A schematic of different potentials associated with colloids in a dispersion is shown in figure 2.5. 


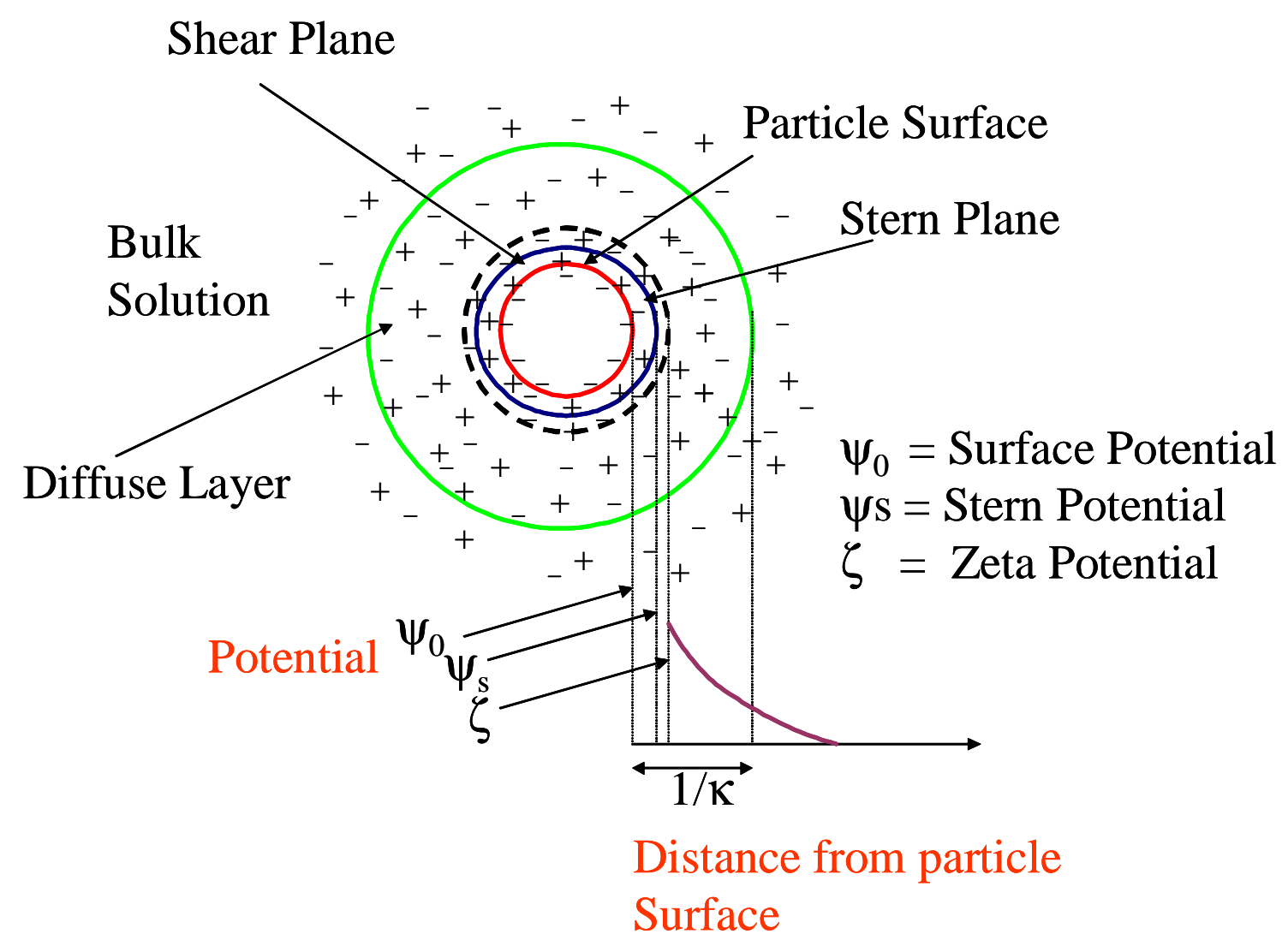

Figure 2.5: Schematic representation of different potentials associated with a particle in aqueous solution [2.23]

The variation of potential in the double layer surrounding a surface of arbitrary shape is given by a Poisson equation:

$\nabla^{2} \psi=-\frac{\rho^{*}}{\varepsilon}$ 
where,

$$
\nabla^{2} \psi=\frac{\partial^{2} \psi}{\partial x^{2}}+\frac{\partial^{2} \psi}{\partial y^{2}}+\frac{\partial^{2} \psi}{\partial z^{2}} \quad \text { (for a flat surface) }
$$

and

$$
\nabla^{2} \psi=\frac{1}{r^{2}} \frac{\partial}{\partial r}\left(r^{2} \frac{\partial \psi}{\partial r}\right)+\frac{1}{r^{2} \sin \theta} \frac{\partial}{\partial \theta}\left(\sin \theta \frac{\partial \psi}{\partial \theta}\right)+\frac{1}{r^{2} \sin ^{2} \theta} \frac{\partial}{\partial \theta}\left(\frac{\partial^{2} \psi}{\partial \phi^{2}}\right)
$$

(for a spherical surface)

In the above equations, $\nabla^{2}$ is the Laplacian operator, $\psi$ is the potential in the double layer as a function of distance from the surface, $\rho^{*}$ is the charge density in the double layer and $\varepsilon$ is the product of $\varepsilon_{0}$, permittivity of free space, and $\varepsilon_{r}$, the relative permittivity between the medium and the free space or vacuum.

Simplified solutions to equation 2.11 are obtained by assuming that the surface potential is less than $25 \mathrm{mV}$, also known as the Debye Huckel approximation. This approximation leads to equations $2.14 \mathrm{a}$ and $2.14 \mathrm{~b}$, which give the variation of potential as a function of distance from a flat and spherical surface respectively [2.24].

$$
\psi=\psi_{0} \exp (-\kappa x)
$$


$\psi=\psi_{0}\left(\frac{a}{r}\right) \exp [-\kappa(r-a)]$

where, $\psi$ is the potential at a distance $x$ or $r$ from the surface, $\psi_{0}$ is the surface potential, $a$ is the radius of the particle and $\kappa$ is the inverse Debye length given by equation $2.14 \mathrm{c}$.

$\kappa=\left[\left(\frac{2000 e^{2} N_{a v}}{\varepsilon k_{B} T}\right) I\right]^{1 / 2}$

where $e$ is the electronic charge, $N_{a v}$ is the Avogadro's number, $k_{B}$ is the Boltzmann constant and $I$ is the ionic strength of the solution.

$\frac{1}{\kappa}$, an important parameter, giving the distance at which the potential drops to $\frac{\psi_{0}}{e}$ and is often referred to as the double layer thickness.

A relation between surface charge $(Q)$ and surface potential can be derived by balancing the charge per unit area on the surface and that in the adjacent solution [2.25]:

$Q=4 \pi \varepsilon_{0} D a(1+\kappa a) \psi_{0}$ 
In deriving equations $2.14 \mathrm{a}$ and $2.14 \mathrm{~b}$, the ions were considered as point charges and therefore there was no limit on the number of ions that can exist close to the charged surface. However, in reality, ions are often surrounded by a hydration shell and can no longer be considered as point charges. J.J. López-García et al. have taken into account the finite sizes of the ions (excluded volume) in determining the potential in the diffuse layer surrounding a charged spherical particle [2.25]. The maximum concentration of counter ions that can surround a high potential surface was limited by using a Langmuir-type correction described in equation 2.16a.

$$
c_{i}(\xi)=\frac{c_{i}^{\infty} \exp \left[-z_{i} y(\xi)\right]}{1+\sum_{i=1}^{m} \frac{c_{i}^{\infty}\left\{\exp \left[-z_{i} y(\xi)\right]-1\right\}}{c_{i}{ }^{\max }}}
$$

where $\xi=\kappa(r-a), a$ being the radius of the particle and $r$ is the distance from the center of particle, $y=\frac{e \phi_{r}}{k T}, \phi_{r}$ is the potential at a distance $r$.

Under these conditions, the potential surrounding a spherical charged surface as a function of radial distance becomes:

$$
y(\xi)=y_{s}-\frac{e \sigma a}{k T \varepsilon}\left(\frac{\xi}{\xi+\kappa a}\right)+\frac{c_{2}{ }^{\max }}{6 c_{2}^{\infty}\left(z_{1}-z_{2}\right)}\left(\frac{\xi^{3}+3 \kappa a \xi^{2}}{\xi+\kappa a}\right)
$$


where $y_{s}=\frac{e \phi_{s}}{k T}, \phi_{s}$ is the surface potential and $\sigma$ is the surface charge density on the spherical surface

Similarly in the case of a planar interface, a more rigorous solution is obtained that eliminates the limitation of the Deybe-Huckel approximation by application of the GouyChapman theory. Equation 2.17a describes the variation in potential with distance from the surface for a diffuse double layer without the simplifying assumption of a low potential [2.13]. The surface potential and surface charge density $\left(\sigma_{\text {surf }}\right)$ can also be correlated by a general equation $2.17 \mathrm{~b}$, applicable for both spherical and planar surfaces $[2.24]$.

$$
\begin{aligned}
& \frac{\left[\exp \left(\frac{z e \psi}{2 k_{B} T}\right)-1\right]}{\left[\exp \left(\frac{z e \psi}{2 k_{B} T}\right)+1\right]}=\left\{\frac{\left[\exp \left(\frac{z e \psi_{0}}{2 k_{B} T}\right)-1\right]}{\left[\exp \left(\frac{z e \psi_{0}}{2 k_{B} T}\right)+1\right]}\right\} \exp (-\kappa x) \\
& \sigma_{\text {surf }}=\left(8 \varepsilon_{0} D k_{B} T n_{\infty}\right)^{1 / 2} \sinh \left(\frac{z e \psi_{0}}{2 k_{B} T}\right)
\end{aligned}
$$

where $n_{\infty}$ is the bulk concentration of ions in number per unit volume. 
Both surface potential and Debye length are important parameters in determining the electrostatic energy of interaction between charged surfaces. Such energies of interaction between a charged sphere and a flat surface with surface potentials $\psi_{01}$ and $\psi_{02}$ respectively is given by equation 2.18 [2.24].

$W_{E}=\pi \varepsilon a\left(\psi_{01}{ }^{2}+\psi_{01}{ }^{2}\right)\left\{\frac{2 \psi_{01} \psi_{01}}{\psi_{01}{ }^{2}+\psi_{01}{ }^{2}} \ln \frac{1-\exp (-\kappa D)}{1+\exp (-\kappa D)}+\ln [1-\exp (-2 \kappa D)]\right\}$

where $W_{E}$ is the electrostatic energy of interaction between the sphere and the surface, $D$ is the shortest distance between the flat surface and the spherical surface, $a$ is radius of the sphere, and $\kappa$ is the inverse Debye length.

\subsubsection{Forces Due to Electro-acoustic Effects in a Megasonic Field}

It is well known that propagation of a sound wave through a dispersion of charged colloids in a medium containing ions results in an alternating electric field [2.27-2.32]. Two mechanisms exist by which an electric field can be formed in a liquid medium irradiated with sonic waves, one requiring only the presence of ions and the other, charged colloids in conjunction with ions. The first one, described by Debye in 1933, is based on the difference in hydrated masses $\left(m_{i}\right)$ and frictional coefficients $\left(\rho_{i}\right)$ of the cations and the anions in the solution and leads to the formation of a potential known as ionic vibration potential (IVP) or Debye potential [2.27]. The IVP oscillates at the same 
frequency as the sound wave along two points in the solution separated by a distance of $\frac{(2 n+1) \lambda}{2}$, where $\lambda$ is the wavelength of the sound wave. A schematic representation of the Debye effect is shown in figure 2.6. The length of the arrows indicates the displacement amplitudes of the ions. The cations due to a smaller hydrated size have larger displacements compared to the anions. As a result, region A is occupied with more cations than anions and hence exhibits a positive potential compared to region $\mathrm{B}$ in the current state of the sound wave shown in the figure. This potential changes in magnitude and direction as the wave propagates through the electrolyte solution.

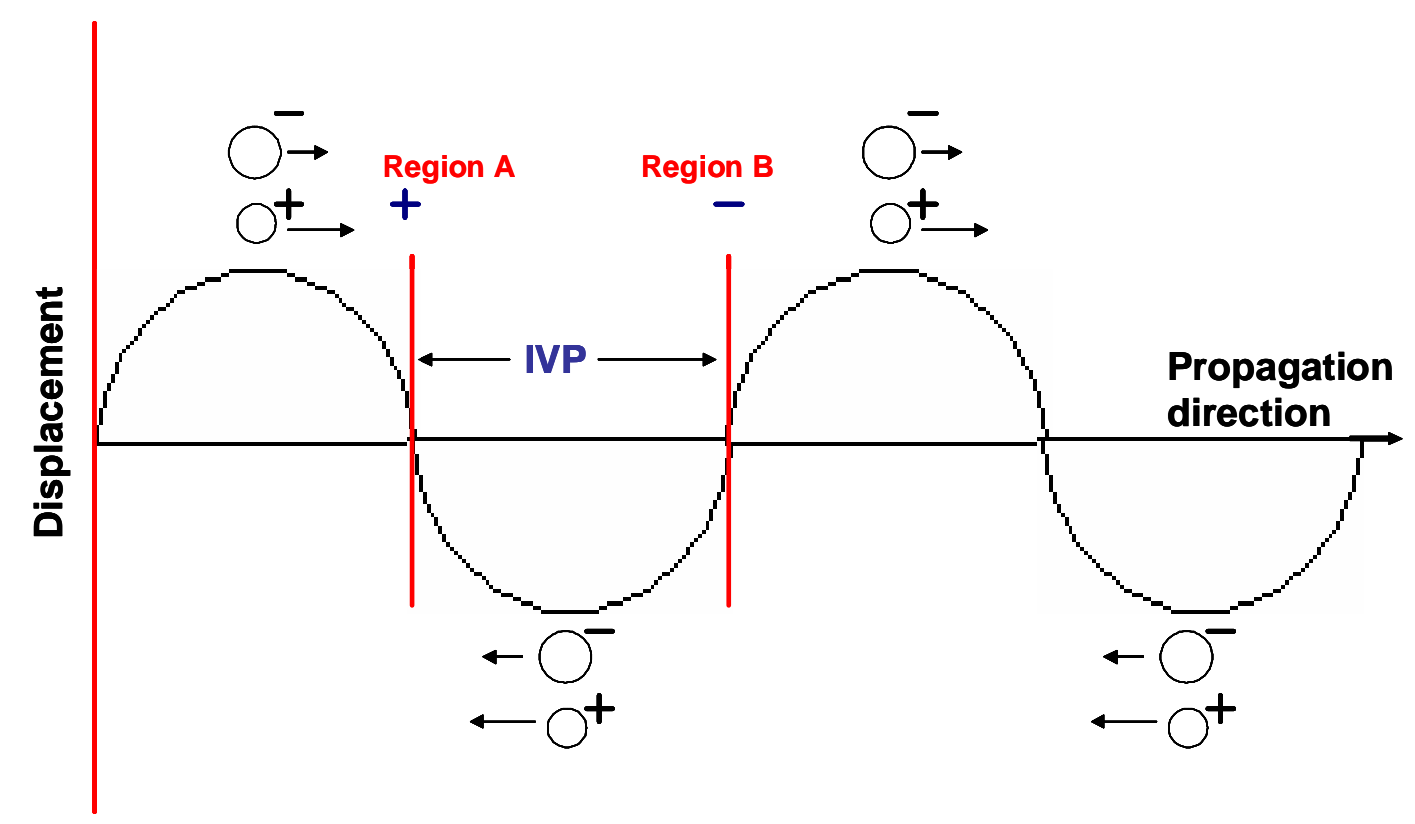

Figure 2.6: Schematic of Debye effect showing formation of IVP due to the difference in displacements of the cations and the anions [2.33] 
In order to be able to obtain the variation of the IVP with time and space, Debye set up three fundamental equations that were solved simultaneously. The first one, shown as (2.19), originates from the equation of motion and balances the inertial force on the ion with frictional force and the electrical force resulting from the separation of the ions.

$$
m_{i} \frac{\partial u_{i}}{\partial t}=-\rho_{i}\left(u_{i}-v\right)+e_{i} X
$$

In the above equation, $u_{i}$ and $v$ are the velocities of ions and solvent respectively and $X$ is the electric field strength. The second equation furnished was the continuity equation which states that any difference between the incoming or outgoing mass through a volume element will result in accumulation of ions in that element, as in (2.20) where $c_{i}$ is the concentration of ion $i$.

$$
\frac{\partial c_{i}}{\partial t}=\frac{\partial\left(c_{i} u_{i}\right)}{\partial x}
$$

Finally Poisson's equation was added as the third equation in the form shown below, which together with equation of motion and continuity was solved for three unknowns, namely $X, u_{i}$ and $c_{i}$.

$\varepsilon_{r}\left(\frac{\partial X}{\partial x}\right)=4 \pi \sum_{i} c_{i} e_{i}$ 
The debye treatment does not include the relaxation effects, hydrodynamic drag and diffusion that are often associated with the motion of ions in the electrolyte solution. A hydrated ion, in an equilibrium position in a charged electrolyte solution, is surrounded by counter and co ions, forming an ionic atmosphere that maintains its electroneutrality. During movement of an ion in an acoustic field, a perturbation in the ionic atmosphere results in a restoring force that provides an electrostatic drag to the motion of the particle [2.34]. This restoring force, computed using equation 2.22 , is referred to as the relaxation force and is illustrated in figure 2.7 [2.35].

$$
F_{i}^{r e l}=e_{i}\left[\frac{e_{1} e_{2} \mid q \kappa X}{3 \varepsilon k_{B} T\left[1+q^{1 / 2}(1+i w \tau)^{1 / 2}\right]}\right]
$$

where $q=\left(e_{1} \rho_{1}^{-1}+e_{2} \rho_{2}^{-1}\right)\left[\left(e_{1}-e_{2}\right)\left(\rho_{1}^{-1}-\rho_{2}^{-1}\right)\right]^{-1}$, suffixes 1 and 2 refer to ions of type 1 and 2 in the electrolyte solution, and $\tau$ is the relaxation time of the ionic atmosphere. 


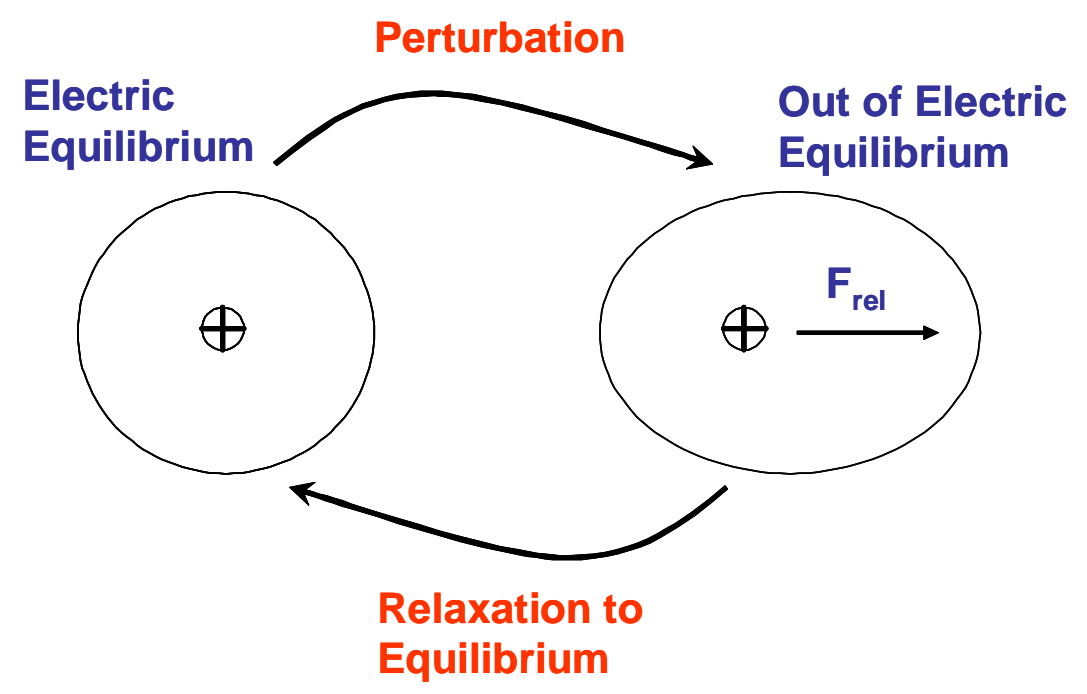

Figure 2.7: Schematic of relaxation force on an ion displaced from its equilibrium in an acoustic field [2.29]

The hydrodynamic drag arises due to the restraining force from the surrounding ions on a moving ion. This force, described in equation 2.23 , is similar in nature to the frictional force described by Debye but includes the effect from the ions especially at high concentrations. The third effect due to diffusion results from the separation of ions in an acoustic field, forming regions of positive and negative ions in the solution. The net effect of diffusion is to reduce the concentration gradient of the ions in the solution as described by equation 2.24. The schematics of hydrodynamic drag and diffusion are shown in figure 2.8. The total effect of relaxation and hydrodynamic forces is less than one percent and is often neglected in the calculations of electric field or potential [2.31]. 
$F_{i}^{h y d}=\frac{e_{i} X \kappa \rho_{i}}{6 \pi \eta_{0}}$

where $\eta_{0}$ is the viscosity of the solvent

$$
F_{i}^{d i f f}=\frac{k_{B} T}{c_{i}} \frac{\partial c_{i}}{\partial x}
$$

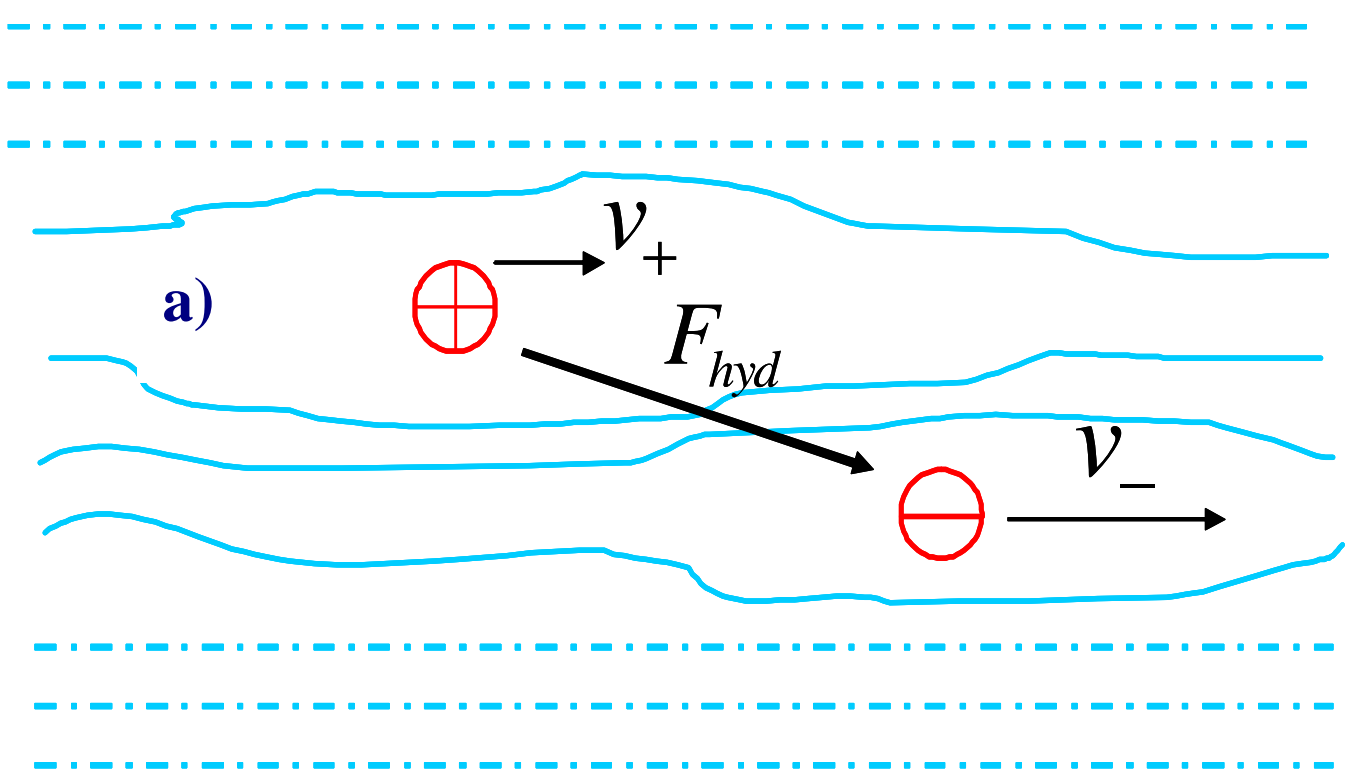




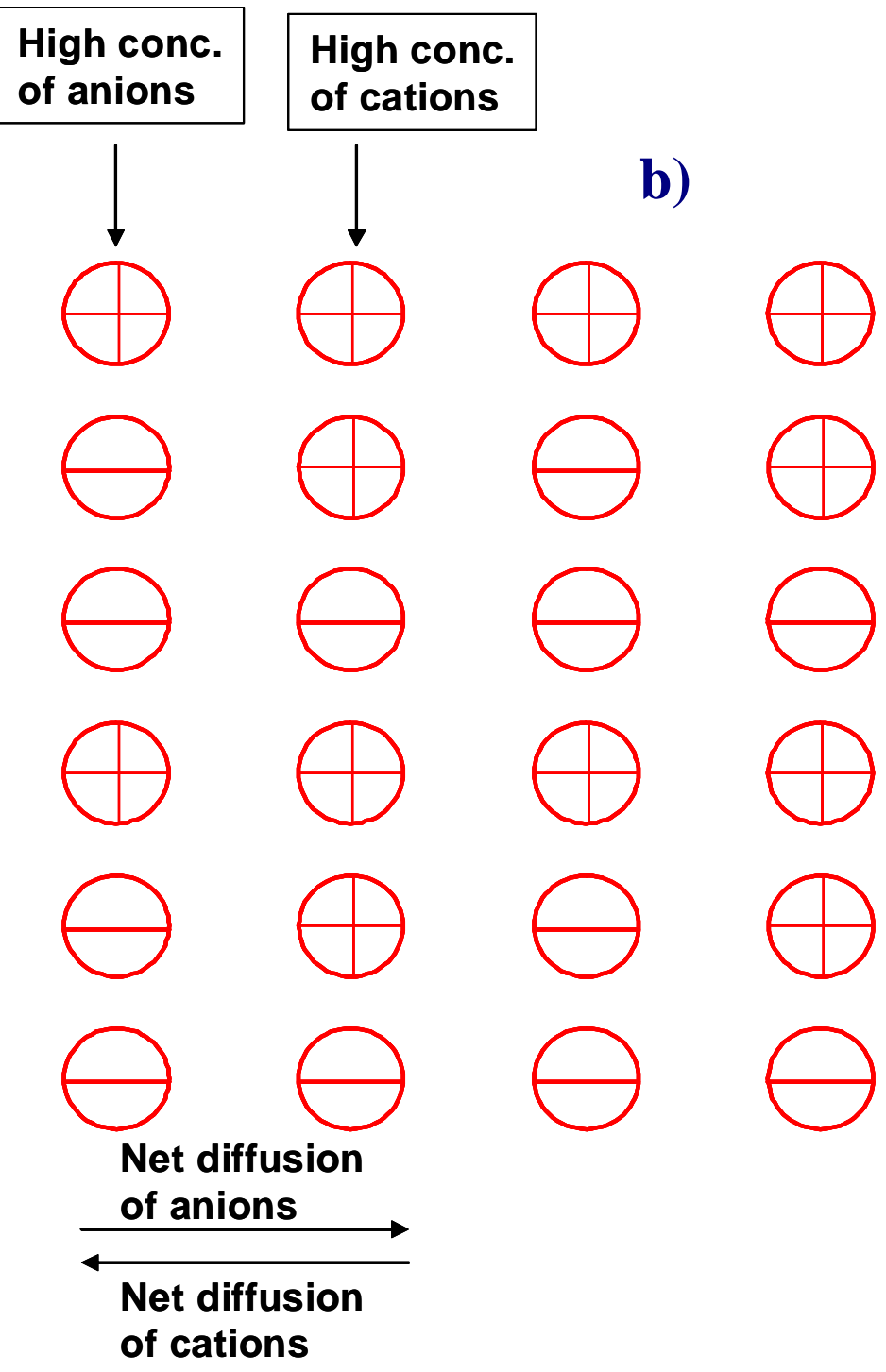

Figure 2.8: a) Hydrodynamic drag on a moving ion due to the surrounding ions b) Net diffusion force due to regions of positive and negative ions [2.29] 
The measurements of IVP have been carried out in solutions of varying ionic strengths exposed to an acoustic field of different intensities [2.28,2.29]. Potentials of the order of 0.3 to $10.2 \mu \mathrm{V}$ have been observed for electrolytes such as lithium chloride, sodium chloride and potassium chloride with concentrations in the range of $10^{-5}-10^{0} \mathrm{M}$ and ultrasonic frequency and power density of $200 \mathrm{kHz}$ and $0.54 \mathrm{~W} / \mathrm{cm}^{2}$ respectively. A theoretical computation of electric field strength, $E$, and ionic vibration potential, $\phi$, can also be made using equations $2.25-2.27$ [2.27].

$$
E=\frac{\delta \phi}{\delta x}
$$

where

$$
\phi=\phi_{0} e^{i(w t-k x)}
$$

and

$$
\phi_{0}=c^{\prime} v_{0} \frac{\left(4 \Pi / \varepsilon_{r} w\right) \sum\left(\overline{c_{j}} m_{j} e_{j} / f_{j}\right)}{\left[1+\left(\left(4 \Pi / \varepsilon_{r} w\right) \sum\left(\overline{c_{j}} e_{j}{ }^{2} / f_{j}\right)\right)^{2}\right]^{0.5}}
$$

In the above equation, $\phi_{0}$ is the maximum amplitude of the potential, $c^{\prime}$ is the speed of sound wave, $v_{0}$ is the maximum velocity of the solvent element, $w$ is the 
frequency of the sound wave, $\overline{c_{j}}$ is the number of ions of $\mathrm{j}^{\text {th }}$ type per unit volume of solution in equilibrium condition, $m_{j}, e_{j}$, and $f_{j}$ are the mass, charge and frictional coefficient of the $\mathrm{j}^{\text {th }}$ ion, $k$ is the ratio of frequency to speed of the sound wave and $\varepsilon_{r}$ is the dielectric constant of the medium.

When charged colloids are present in the ionic medium, the IVP is often accompanied by an electrokinetic effect that produces a potential named colloid vibration potential, CVP. This effect, first reported by Hermans and Rutgers [2.30-2.32], occurs due to the relative displacement between the particle and the surrounding ions in the double layer leading to formation of a dipole moment. Enderby used a mathematical treatment to obtain expressions for dipole moment and potential due to this moment at distances much larger than the double layer thickness [2.36]. The following equations of motions and continuity along with Poisson's equation were solved for ions and the solvent molecules in the electrolyte solution by employing a method of successive approximations as described below:

$f_{i}\left(u_{i}-v\right)+e z_{i} \nabla \psi+\frac{k T}{c_{i}} \nabla c_{i}+m_{i} \frac{\partial u_{i}}{\partial t}=0$

$\frac{\partial c_{i}}{\partial t}+\nabla \cdot\left(c_{i} u_{i}\right)=0$ 
$\rho_{0} \frac{\partial v}{\partial t}=B \rho_{0}-\rho_{0}(v \cdot \nabla v)-\nabla p+\frac{\eta \nabla(\nabla \cdot v)}{3}+\eta \nabla^{2} v$

$B \rho_{0}=e z_{i} c_{i} \nabla \psi+k T \nabla c_{i}+m_{i} c_{i} \frac{\partial u_{i}}{\partial t}$

$\frac{\partial \rho_{0}}{\partial t}=\nabla \cdot\left(\rho_{0} v\right)$

$\nabla_{2} \psi=-\frac{4 \pi e}{\varepsilon} \sum_{i=1}^{n} c_{i} z_{i}$

In the above equations, $u_{i}$ and $v$ are the velocities of the ion of type $i$ and solvent molecules respectively, $c_{i}$ is the concentration of ions in the solution, $\phi$ is the potential, $p$ is the pressure in the solution, $\rho_{0}$ and $\eta$ are the density and viscosity of the solution.

Since the region of interest is close to the particle surface, Enderby used Stokes approximation and neglected the inertial terms in the equation of motion. First, distribution of ions and a potential around a charged particle in an undisturbed state of fluid $\left(u_{i}=0\right)$ was obtained by solving equations 2.28 and 2.33 simultaneously. These were substituted in equations 2.30 and 2.32 , where $B \rho_{0}$ was first taken as zero, and the solvent molecules velocity profile was computed. Using this value of solvent velocity in equations 2.28 and 2.29, new expressions of potential and ion velocity were obtained. By 
substituting these solutions for potential and ion velocity, another value for solvent velocity was computed which was further used to obtain better values of potential and ion velocity. The equation for dipole moment was derived using this procedure, and its expression is shown below in 2.34 .

$$
\mu=-\frac{4 \pi g q v Q}{\kappa^{2} k T(1+b)}\left\{G(b, \gamma)+\left(\frac{2 \pi}{\kappa \lambda}\right)^{2} H(b, \gamma)\right\}
$$

where $g=\frac{2(1-\rho)}{1+\frac{9}{\gamma}+\frac{9}{\gamma^{2}}+2 \rho}, G(b, \gamma)=\frac{(1+\gamma) B(b)}{96}, q=\frac{\sum f_{i} c_{i} z_{i}^{2}}{\sum c_{i} z_{i}^{2}}$

$B(b)=48\left(3-\frac{9}{b}+\frac{75}{b^{2}}-\frac{660}{b^{3}}+\frac{6300}{b^{4}}-\frac{65420}{b^{5}}+\cdots\right), Q$ is the surface charge on the particle also equal to $\varepsilon a \zeta(1+b), a$ is the radius of the particle, $\zeta$ is the zeta potential, $b$ is the ratio of particle radius to double layer thickness, $\rho$ is the ratio of solid density $\left(\rho_{1}\right)$ to fluid density $\rho_{0}, \gamma=\left(2 s^{2}(1-i)\right)^{1 / 2}, 2 s^{2}=\frac{\rho_{0} w a^{2}}{\eta}, w$ is the frequency of the sound wave in $\mathrm{rad} / \mathrm{s}$, and $\eta$ is the viscosity of the solution.

The term containing $H(b, \gamma)$ is much smaller compared to $G(b, \gamma)$, except at very high frequencies, and hence was neglected. This simplified the equation of dipole moment as: 
$\mu=-\frac{4 \pi g q v Q}{\kappa^{2} k T(1+b)} G(b, \gamma)$

The potential due to this dipole at distances larger than double layer thickness can be computed as:

$$
\phi=\left(\frac{\mu}{4 \pi \varepsilon}\right) \frac{\cos \theta}{r^{2}}
$$

Marlow et al. followed Enderby's approach and obtained the dipole moment maximum amplitude $(m)$ in a slightly different form [2.33].

$$
m=\frac{6 \pi \varepsilon_{0}{ }^{2} D^{2} \varsigma v a f(\chi a)}{\alpha}
$$

where $\varepsilon_{0}$ is the permittivity of free space, $\varsigma$ is the zeta potential of the particle, $v$ is the maximum difference in velocity between the particle and the fluid, $a$ is the particle radius, $\alpha$ is the conductivity of the solution, $\chi$ is the double layer thickness, and $f(\chi a)$ is a correction factor varying from $2 / 3$ to 1 , depending on the relative dimensions of double layer and particle radius. 
The magnitude of difference in velocity between the particle and the fluid, $v$, can be obtained by a force balance between the viscous and inertial forces experienced by the particle as shown in equation 2.38 .

$$
v=\frac{2 a^{2}\left(\rho_{2}-\rho_{1}\right)(\omega U)}{9 \eta}
$$

where $\rho_{2}$ is the density of the particle, $\rho_{1}$ is the density of the fluid, $\eta$ is the viscosity of the fluid, and $U$ is the maximum velocity of the fluid in a plane monochromatic sound wave traveling in the $x$ direction. The term $U$ is related to the transducer intensity, $I$, as

$$
U=\left(\frac{2 I}{\rho_{1} c}\right)^{0.5}
$$

As evident from equations 2.37 and 2.38, the dipole moment increases with the difference in density between the particle and the fluid, size and the zeta potential of the particle and the transducer power density. Equation 2.37 also suggests that the dipole moment decreases with an increase in the conductivity of the solution. This conductivity effect can be explained as follows. When the ions in the double layer move relative to the particle, there is a backflow of ions in the continuous phase to make the overall charge transport zero. The higher the conductivity, the higher will be the backflow of ions to restore equilibrium. This reduces the dipole moment. In the case where backflow of ions is small or absent, equation 2.37 can be reduced to equation 2.40 [2.33]. 


$$
m=\frac{6 \pi v a \varsigma \varepsilon_{0} D}{w}
$$

The relative motion between the particle and the surrounding ions causes the center of the charges between the particle and the double layer to become offset, by a distance $l$, from its initial position. Due to the resulting offset between the centers of two charges, the particle experiences an attractive force, $F$, in the direction of the offset. In an acoustic field, this force can be expected to oscillate and a simple model can be used to estimate the maximum amplitude of this force as [2.37]:

$$
F=\frac{m Q^{\prime}}{l^{3}}=\frac{Q^{\prime 2}}{l^{2}}
$$

where $Q^{\prime}$ is the net charge due to shift in the center of charges and $l$ is the offset between the center of particle and the double layer.

In a dilute colloidal suspension, where each colloid can be considered to be isolated or non-interacting with other colloids, a microscopic potential is developed around each particle which varies inversely as the square of the distance from the center of the particle $(r)$ at large distances from the particle. This potential is related to the dipole moment by the simple expression as shown below [2.33]: 
$\psi=\frac{m \cos \theta}{4 \pi \varepsilon_{0} D r^{2}}$

where $\psi$ is the maximum microscopic perturbation potential, and $\theta$ is the angle between the z-axis (in the direction of motion of sound waves) and the radius vector $r$.

The microscopic potential around each colloid collectively forms a macroscopic potential in a suspension of $N$ colloids per unit volume, which oscillates sinusoidally across two points separated by half a wavelength and is given by [2.33]:

$$
\Phi=\frac{9 \lambda \varepsilon_{0} D \varsigma \phi v f(\chi a)}{2 \pi a^{2} \alpha}
$$

where $\Phi$ represents the peak value of the CVP, $\lambda$ is the wavelength of the sound wave and $\phi$ is the volume fraction of the non-interacting colloids and is equal to $\frac{4 \pi a^{3} N}{3}$

Experimental measurements of CVP have shown that its magnitude is much larger than IVP and the summation of the two is often used to obtain the total vibration potential (TVP) [2.38]. The first experimental work on the CVP was performed by Yeager et al. on silica suspensions subjected to $0.2 \mathrm{MHz}$ sound wave frequency [2.39]. The measured values of CVPs per unit fluid velocity in the particle concentration range of 0.85 to 26.8 weight percent and solution specific conductance of $14 \times 10^{-4}$ to $204 \times 10^{-4} \mathrm{~S} / \mathrm{m}$ were 
found to be between $0.88 \mathrm{mV}$ and $1.7 \mathrm{mV}$ per $\mathrm{cm} / \mathrm{s}$, corresponding to CVPs of 4.7 and 9 $\mathrm{mV}$ respectively at a transducer power density of $0.22 \mathrm{~W} / \mathrm{cm}^{2}$. Y. Hozumi et al. obtained CVP measurements in a silica dispersion containing $\mathrm{KCl}$ at a concentration of $10^{-3} \mathrm{~mol} / \mathrm{lit}$ maintained at a solution $\mathrm{pH}$ of 5.0. The measured CVP at a sound wave frequency of 0.2 $\mathrm{MHz}$ ranged from $0.1 \mathrm{mV}$ to $3.5 \mathrm{mV}$ for particle volume fraction of between 0.005 to 0.3 $[2.40]$.

Although significant work has been done on experimental and theoretical determination of IVP and CVP and their associated electric fields, the effect of these potentials on particle removal has never been investigated.

\subsection{Wave Acoustics}

In order to have a clear understanding of megasonic cleaning, the basics of acoustics including sound wave propagation, attenuation of sound waves due to viscous and thermal losses, cavitation, and acoustic streaming have to be understood. A short review of some of the important topics in wave acoustics relevant to megasonic cleaning is presented in the following section. 


\subsubsection{Sound Wave Propagation}

A sound wave is a pressure disturbance that travels through a medium by particle to particle interaction. As one particle is displaced it exerts a force on the adjacent particle, thus disturbing that particle from its equilibrium position and transferring energy through the medium. In a perfect fluid where viscous forces can be neglected, the above motion of a particle in a fluid can be described by a wave equation expressed in 2.44 and 2.45 , using equations of continuity and motion [2.41].

$\frac{\partial^{2} p}{\partial t^{2}}=c^{2}\left(\frac{\partial^{2} p}{\partial x^{2}}+\frac{\partial^{2} p}{\partial y^{2}}+\frac{\partial^{2} p}{\partial z^{2}}\right)$

or

$$
\frac{\partial^{2} \mathfrak{I}}{\partial t^{2}}=c^{2}\left(\frac{\partial^{2} \mathfrak{I}}{\partial x^{2}}+\frac{\partial^{2} \mathfrak{I}}{\partial y^{2}}+\frac{\partial^{2} \mathfrak{I}}{\partial z^{2}}\right)
$$

where $p$ is the sound wave pressure in the medium as a function of time $t$ and position coordinates $x, y$ and $z, c$ is the speed of sound equal to $\left(\frac{\kappa}{\rho}\right)^{0.5}, \kappa$ and $\rho$ are the fluid bulk modulus and density respectively. The property $\mathfrak{I}$ represents fluid element displacements from the equilibrium position either in $x, y$ or $z$ directions and may also 
be replaced by particle velocities $u_{x}, u_{y}$ or $u_{z}$ provided the motion of the fluid is irrotational.

The transmission of a sound wave through a medium involves both kinetic and potential energy. Since frictional heat losses are neglected in a non-viscous flow, the energy content of the sound wave remains unchanged. The kinetic and potential energies crossing a unit area normal to the direction of propagation in unit time, also defined as intensity $I$, for a progressive plane wave in $x$ direction is given by equation 2.46 [2.42].

$$
I=c \rho_{0} \overline{u_{x}^{2}}=\frac{\overline{p^{2}}}{\rho_{0} c}
$$

where the bar over a quantity denotes the time average of the quantity, and $\rho_{0}$ is the density of the medium when no wave is propagating.

In a plane progressive wave that is also harmonic, the pressure is a sinusoidal function of the time with maximum value $a$. Since the average value of $\sin ^{2} \theta$ over a complete period is $1 / 2, \overline{p^{2}}=\frac{a^{2}}{2}$, this transforms the intensity equation as:

$$
I=\frac{a^{2}}{2 \rho_{0} c}
$$


Since, $c=\left(\frac{\kappa}{\rho}\right)^{0.5}$

$a=(2 I)^{0.5}\left(\rho_{0} \kappa\right)^{0.25}$

Equation 2.48 can be used to compute the pressure amplitude of the sound wave if intensity of the sound source (transducer power density) and solution properties are known.

In addition, one can also calculate the velocity amplitude $\left(u_{x}\right)$, and acceleration amplitude $\left(a_{t}\right)$ of the fluid particle using the sound wave pressure amplitude as shown below [2.43].

$u_{x}=\frac{p}{\rho_{0} c}$

$a_{t}=\frac{w p}{\rho_{0} c}$

where $w=2 \pi f$, and $f$ is the frequency of sound wave in cycles per sec. 
The computation of these parameters is often performed to characterize the sound field and to obtain useful information on acoustic related effects such as cavitation, and streaming, as discussed in later sections.

\subsubsection{Attenuation}

In a viscous fluid, the plane wave sound intensity decreases with distance due to the effect of shear and compression viscosity of the fluid. The shear viscosity incorporates any frictional resistance arising from a change in the shape of a volume element as stated in Stokes' hypothesis. By contrast, the compression viscosity losses originate due to the volume changes even when the shape remains unaltered. Sound wave attenuation due to the shear viscosity $(\mu)$ effect can be included in the wave equation by adding a viscous term as expressed in equation 2.52 [2.42].

$$
\frac{\partial^{2} p}{\partial t^{2}}=c^{2}\left(\frac{\partial^{2} p}{\partial x^{2}}\right)+\frac{4 \mu}{3 \rho_{0}} \frac{\partial^{2} p}{\partial x \partial t}
$$

The solution to this equation for harmonic vibrations in a perfect fluid $(\mu=0)$ is given by:

$p=a e^{i w\left(t-\frac{x}{c}\right)}$ 
As one can observe from this equation, there is no decay in the pressure amplitude of the sound wave with distance. However, when viscous effects are included, the pressure equation takes the form:

$$
p=a e^{i w\left(t-\frac{x}{c}\right)} e^{-\alpha x}
$$

where, the coefficient of attenuation, $\alpha=\frac{8 \pi^{2} \mu f^{2}}{3 \rho_{0} c^{3}}$,

The term $e^{-\alpha x}$, referred to as the damping factor, decreases exponentially with distance. Since $\alpha$ is proportional to the square of the frequency, the attenuation increases with the frequency of the sound wave.

Assuming a transducer intensity of $2.17 \mathrm{~W} / \mathrm{cm}^{2}$, acoustic frequency of $0.925 \mathrm{MHz}$, speed of sound in DI water as $1480 \mathrm{~m} / \mathrm{s}$ and water density and viscosity of $10^{3} \mathrm{Kg} / \mathrm{m}^{3}$ and $8 \times 10^{-4} \mathrm{~N}-\mathrm{s} / \mathrm{m}^{2}$ respectively at $25{ }^{0} \mathrm{C}$, the pressure amplitude $\left(a e^{-\alpha x}\right)$ can be plotted as a function of distance as shown in figure 2.9. The data in the graph indicate that the pressure amplitude decreases from 2.49 atm to 2.11 atm when the sound wave traverses a distance of $30 \mathrm{~m}$. In a megasonic cleaning tank of about $30 \mathrm{~cm}$ in height, similar calculations show that the pressure amplitude decreases across the height of the tank by 0.003 atm in DI water. This decrease may be considered small and hence the shear 
viscosity effect in the calculations of fluid element velocity, and acceleration (equations 2.50-2.51) may be neglected without any considerable error.

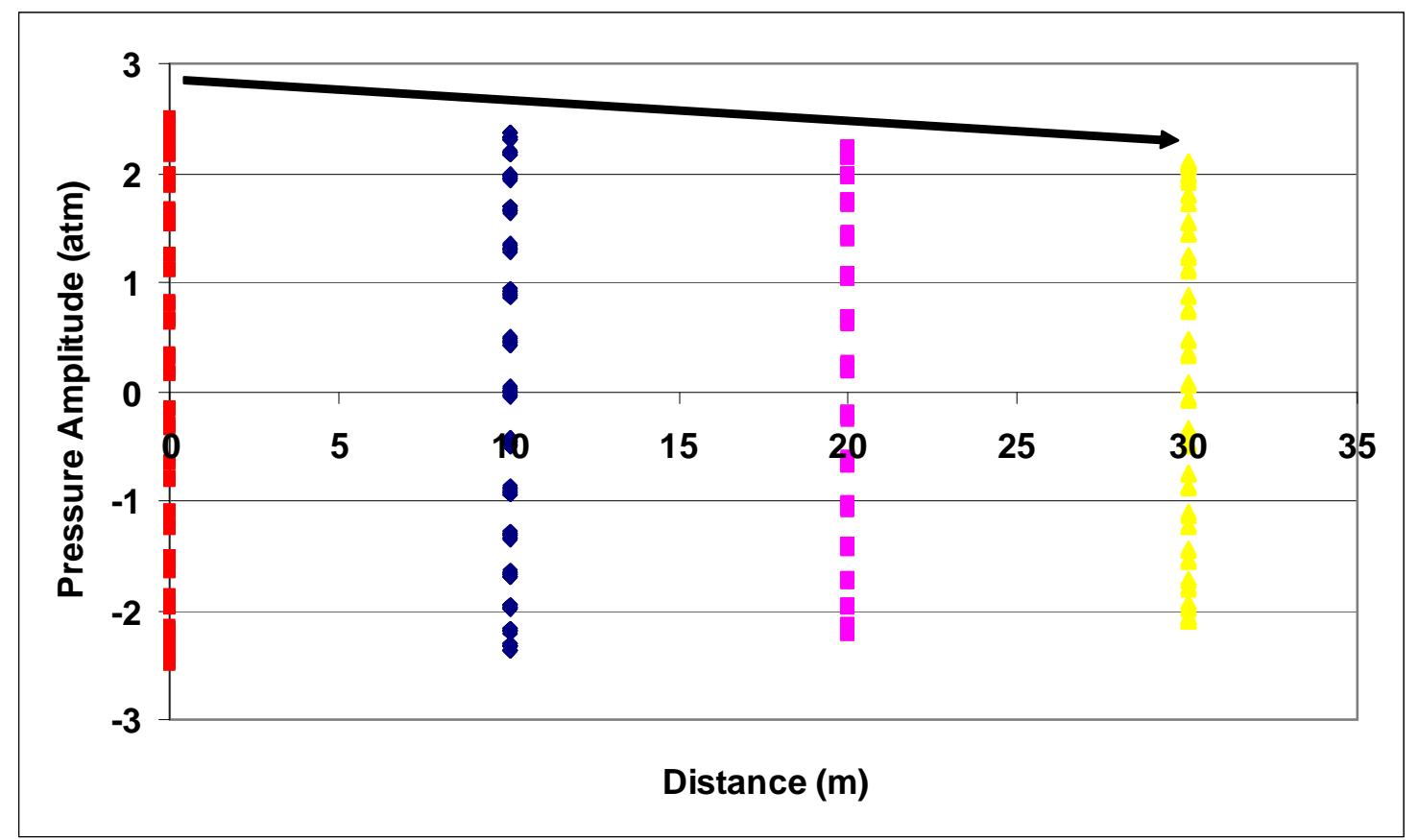

Figure 2.9: The decay in pressure amplitude of sound wave with distance in a viscous medium

Attenuation due to thermal conduction is sometimes considered when energy loss occurs due to transfer of heat from hot regions (compressions) to the colder regions (rarefactions) created by the sound wave [2.41]. In such a case, the coefficient of attenuation gets modified as 
$\alpha=\frac{8 \pi^{2} f^{2}}{3 \rho_{0} c^{3}}\left(\mu+\frac{3 \hbar(\gamma-1)}{4 C_{p}}\right)$

where $\lambda$ is the thermal conductivity of fluid, $C_{p}$ is the isobaric heat capacity and $\gamma$ is the ratio of heat capacities at constant pressure and volume.

In the case of water, $\frac{3 \lambda(\gamma-1)}{4 C_{p}}=1.2 \times 10^{-6} \frac{K g}{m-\mathrm{sec}}$ for $\lambda=0.6 \frac{\mathrm{W}}{\mathrm{mK}}, \gamma=1.011$ and $C_{p}=4181 \frac{J}{K g K}$ at $25{ }^{0} \mathrm{C}$. The thermal conduction term is much smaller than $\mu$ for water, hence attenuation basically remains unchanged in water, even with the inclusion of thermal conduction. 


\subsubsection{Cavitation}

\subsubsection{Introduction}

Cavitation may be defined as stimulated bubble (or cavity) activity in a liquid [2.44]. When the bubble activity is induced by acoustic waves, it is referred to as acoustic cavitation. This bubble activity is known to produce chemical and physical effects such as acoustic streaming, shock waves, fluid jet formation, chemical reactions, radiation forces, and erosion. In turn, these effects drive applications and processes such as ultrasonic and megasonic cleaning, sonochemistry (improved reaction efficiency when ultrasound is applied through the generation of free radicals and enhanced mixing) and sonocrystallisation (in which collapsing bubbles form 'seed sites' from which crystals can grow in a controlled manner). In order to form a bubble in a liquid, a reduction in liquid pressure is required, which can be achieved by sending a sound wave through a medium. The tensile strength of pure water at $25{ }^{0} \mathrm{C}$ is about $1000 \mathrm{~atm}$. This means that sound wave pressure amplitude of at least 1000 atm is required to initiate cavitation in water, assuming no other nuclei are present [2.45,2.46]. However, cavitation is observed in liquids with pressure amplitudes as low as $1 \mathrm{~atm}$, which suggests the pre-existence of nuclei within the liquid. Thus the threshold pressure for inception of bubbles in liquid can be significantly lower than the theoretically predicted values depending on several factors including gas trapped in crevices or cracks of solid particles, organic skins around bubbles, partially wetted dirt particles and others. 
Once a gas bubble is formed, it can either grow into a stable bubble or into a much larger bubble, known as a transient bubble, which collapses violently in a time scale of a few microseconds or less depending on the frequency of the acoustic wave. It is also possible that the bubble may dissolve away in the liquid due to diffusion of gas from the bubble into the liquid, especially if its size is extremely small such that surface tension forces dominate. The stable oscillating bubbles often grow in size due to net diffusion of gas into the bubble (also known as rectification) or by coalescence, and become transient cavities which immediately explode and disintegrate into smaller bubbles. In addition, stable cavitation is also associated with some of the important effects such as microstreaming and surface oscillations which have been studied extensively because of their wide applications. Thus, the process of cavitation can be divided into stable and transient cavitation, both of which are described briefly in the following sections.

\subsubsection{Stable Cavitation}

Stable cavitation has been investigated in detail by Coakley and Nyborg [2.47]. Although stable cavities do not collapse or lead to extreme temperature and pressure conditions in the solution, more often than not they transform into transient cavities. Hence, knowledge of the distribution of stable bubbles is important in determining the extent of transient cavitation. In the case of gas bubbles, a linear theory of small oscillations can be used to predict the size distribution of bubbles in liquids if information on the frequency of oscillation of bubbles is available [2.48]. This theory assumes that the 
relative changes in bubble volume during a bubble's expansion and contraction are small. Since the kinetic energy of the bubble is much smaller than that of the surrounding liquid due to low density of the bubble, it can be ignored. Similarly since the liquid is incompressible, its potential energy is negligible compared to that of the bubble. It follows that rate of work done by the external medium (say transducers) minus the viscous forces is equal to the rate of change of sum of the kinetic energy of the liquid and potential energy of the bubble. This leads to two significant correlations between the resonance frequencies ( $w$ in $\mathrm{rad} / \mathrm{sec}$ ) of oscillation of bubbles and their respective sizes $(R)$ as expressed in equations 2.56 and 2.57. Equation 2.56 applies to larger bubbles (say 30 micron or larger) where surface tension forces can be ignored. In this case, the inverse relation between the bubble size and frequency is linear. For smaller bubbles, equation 2.57 must be used which takes into account the surface tension of the liquid $(\sigma)$. A plot of frequency of air bubbles $\left(\gamma=1.4\right.$ for air) in water $\left(\rho=10^{3} \mathrm{Kg} / \mathrm{m}^{3}\right)$ versus their size for initial liquid pressure $\left(P_{0}\right)$ of 1 atm is shown in figure 2.10 .

$$
w^{2}=\frac{3 \gamma P_{0}}{\rho R^{2}}
$$

$$
w^{2}=\frac{1}{\rho R^{2}}\left[3 \gamma\left(P_{0}+\frac{2 \sigma}{R}\right)-\frac{2 \sigma}{R}\right]
$$




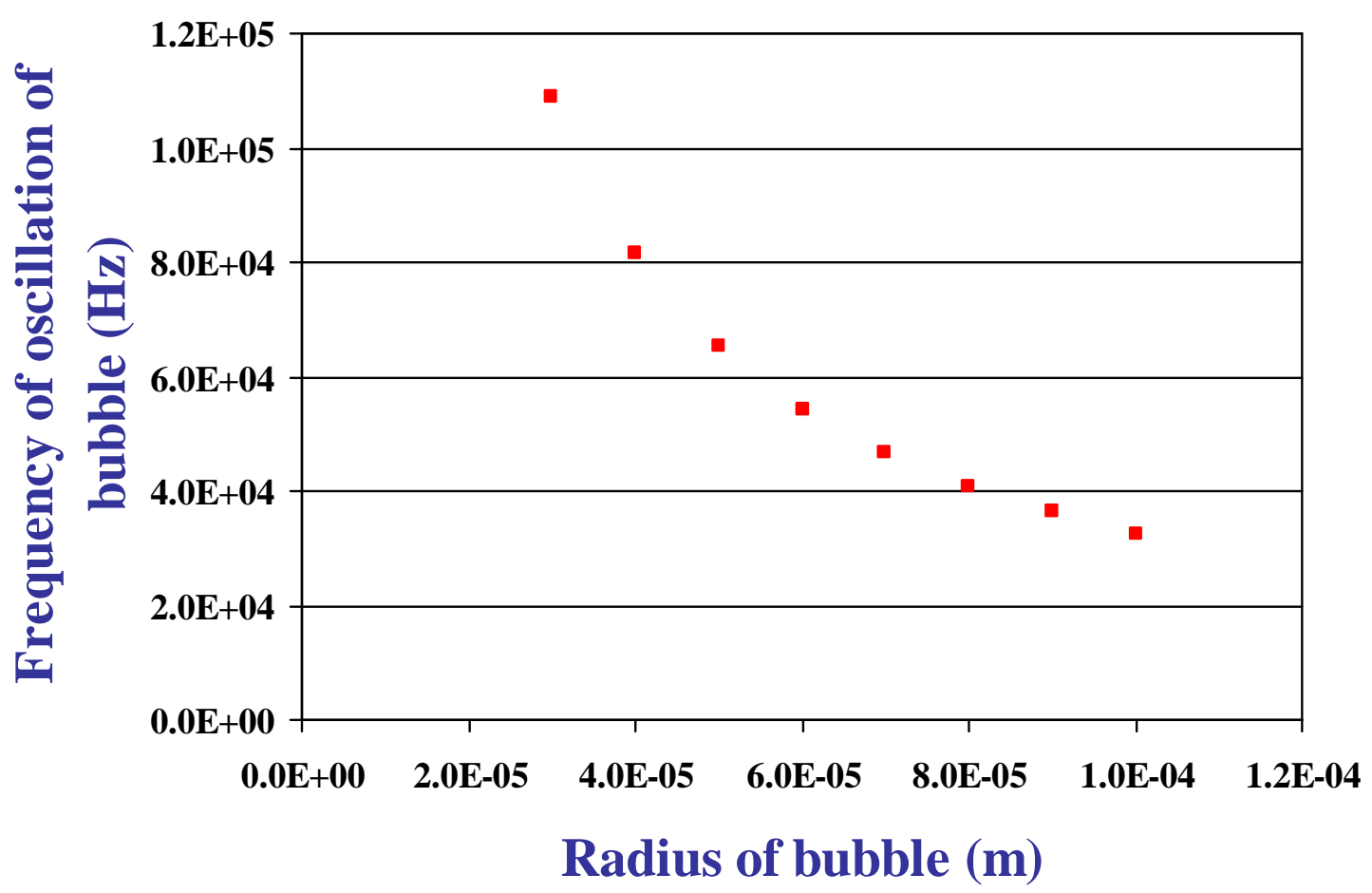

Figure 2.10: Radius of oscillating bubbles versus frequency for large bubbles (surface tension forces insignificant)

Lauterborn obtained a numerical solution of non-linear oscillations of stable cavities in liquids [2.49]. When a bubble is driven at a frequency such that the ratio of driving frequency to resonance frequency is a rational fraction (i.e. $w_{d} / w_{r}=\mathrm{n} / \mathrm{m}$ where $\mathrm{n}$ and $\mathrm{m}$ are integers), it can generate periodic motions which are subharmonics $(\mathrm{n}=1$, $\mathrm{m}=1,2,3 \ldots)$, harmonics $(\mathrm{m}=1, \mathrm{n}=1,2,3 \ldots)$ or ultraharmonics $(\mathrm{n}=2,3 \ldots, \mathrm{m}=2,3 \ldots)$ of the driving frequency. For example, in the case of $w_{d} / w_{r}=4 / 5$ or $w_{d} / w_{r}=9 / 5$ with the source pressure amplitude equal to one third of the equilibrium pressure, subharmonics at $w_{d} / 4$ and $w_{d} / 9$ respectively were produced [2.50]. Figure 2.11 shows a bubble radius time curve and the frequency spectrum for a bubble radius of $10 \mu \mathrm{m}$ in a pressure field of 0.7 
bar amplitude and driving frequency of $122.66 \mathrm{KHz}$. One can notice ultraharmonics on the order of $5 / 2$ since five maxima are present in two cycles of the driving sound field.
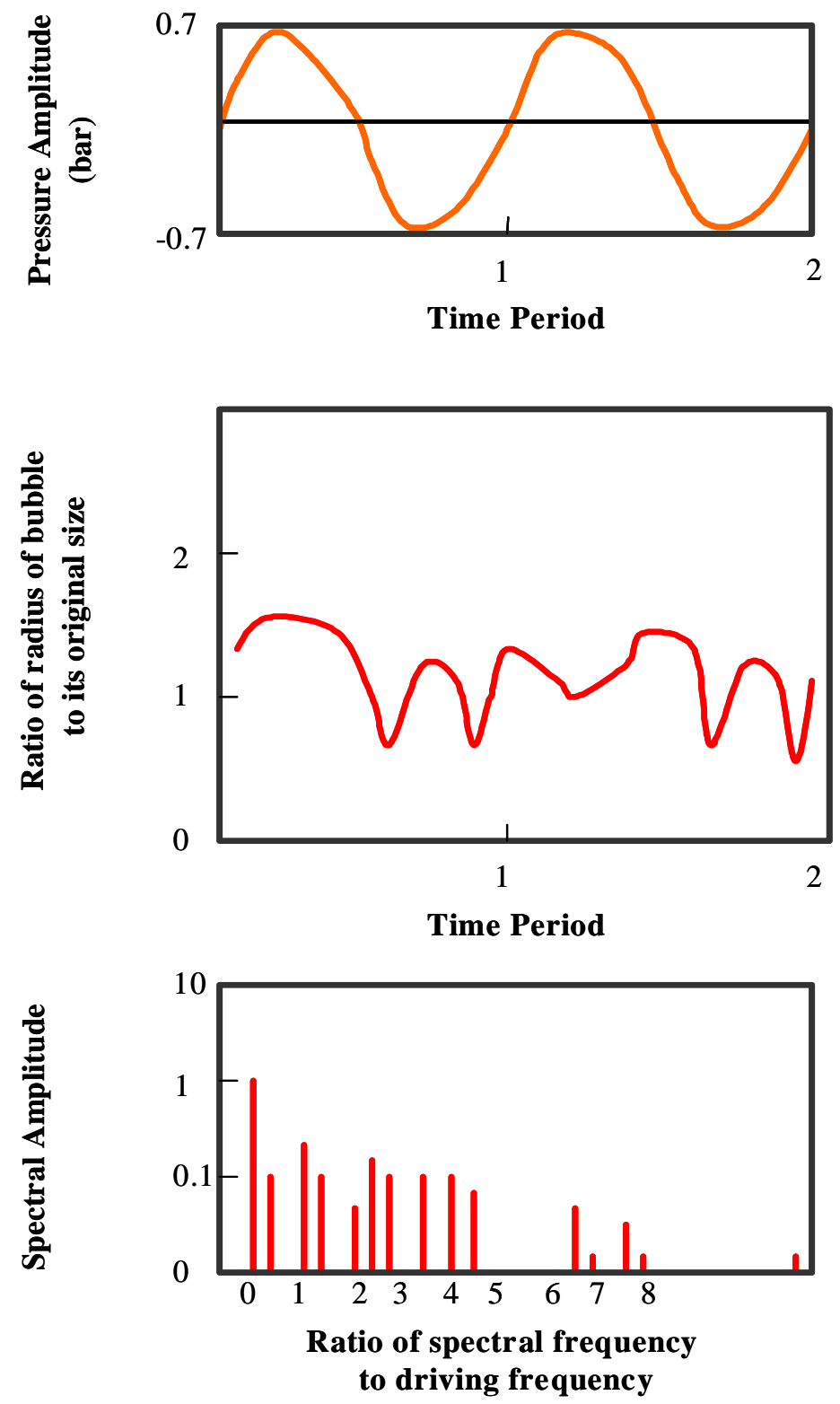

Figure 2.11: Non-linear oscillation of stable gas bubbles showing ultraharmonics of the driving harmonics [2.49] 


\subsubsection{Transient Cavitation}

In order to access the likelihood of transient cavitation, it is necessary to know the 'threshold pressure' or minimum pressure for growth and subsequent collapse of a bubble to maximum temperature and pressure. Blake predicted the minimum threshold pressure, known as the Blake Threshold Pressure, required for explosive growth of a gas bubble as a function of bubble initial radius (Blake Radius) as given by equation 2.58 [2.48, 2.51]. Inertial and viscous forces were ignored in deriving the threshold pressure and therefore this equation is strictly valid only for smaller bubbles where surface tension forces are

dominant. A plot of Blake threshold pressure versus initial bubble radius for water and a mixture of ethylene glycol and ethanol is shown in figure 2.12 [2.52]. The Blake threshold for water is higher than that for a mixture of ethylene glycol and ethanol due to the higher surface tension of water. It will be clear later that Blake's model holds good for any bubble radius below $0.15 \mu \mathrm{m}$.

$$
P_{B}=P_{0}+\frac{8 \sigma}{9}\left[\frac{3 \sigma}{2\left[P_{0}+\left(\frac{2 \sigma}{R_{B}}\right)\right] R_{B}{ }^{3}}\right]^{1 / 2}
$$

where $P_{B}$ is the Blake threshold pressure, $\sigma$ is the surface tension of the liquid, $P_{0}$ is the equilibrium pressure of the liquid, and $R_{B}$ is the initial gas bubble radius. 


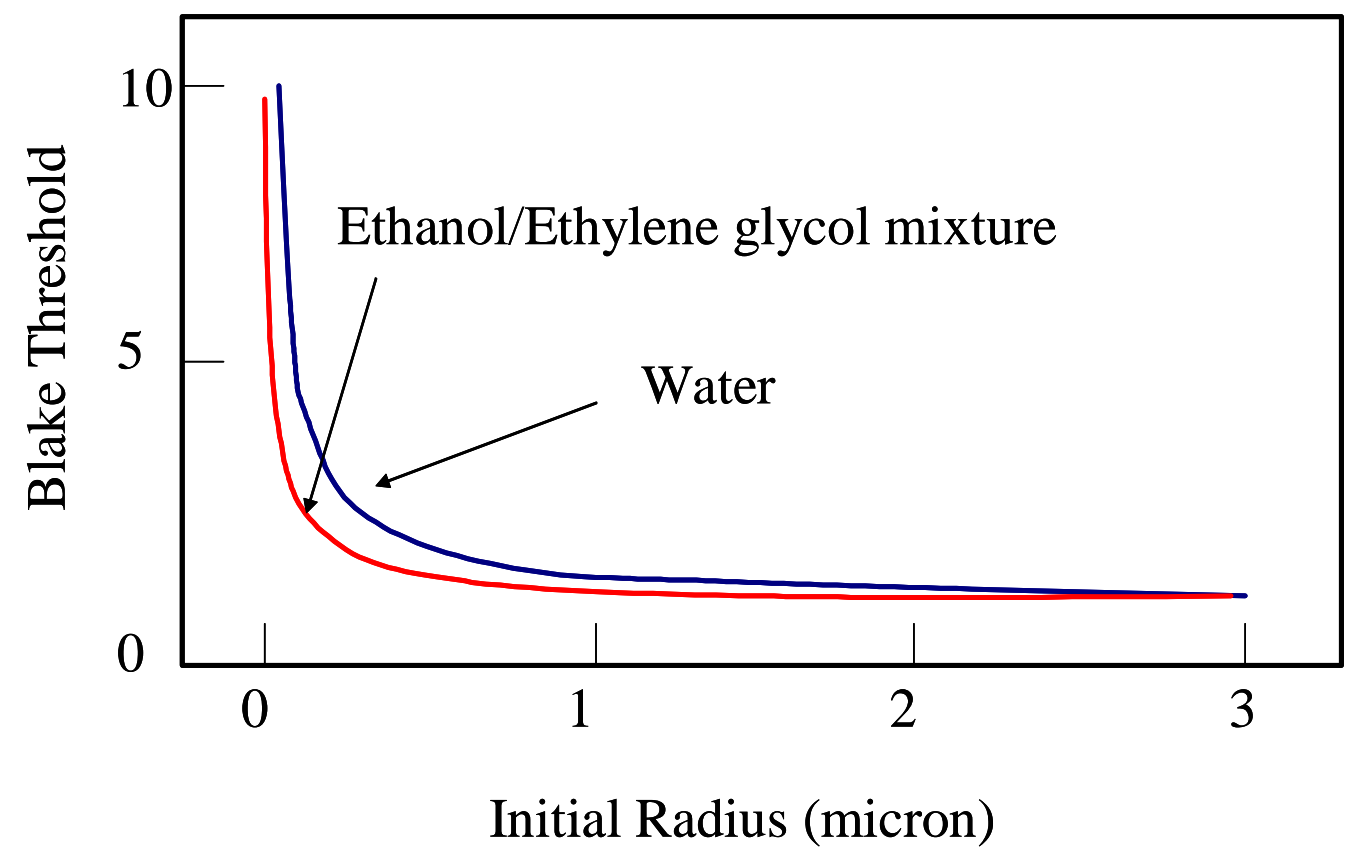

Figure 2.12: Blake threshold pressure for water and a mixture of ethylene glycol and ethanol as a function of initial bubble radius [2.52]

In order to be able to incorporate the effect of inertial and viscous forces in bubble dynamics, Apfel gave a simple derivation based on a first law energy balance [2.53]. The sum of energy (pressure energy) supplied by the sources and the work done by surface tension should balance the kinetic energy of the liquid, viscous energy dissipation at the bubble surface and the increase in pressure energy of the bubble. This energy balance will result in the following fundamental equation of bubble dynamics, also known as the Rayleigh-Plesset equation.

$R \frac{d^{2} R}{d t^{2}}+\frac{3}{2}\left(\frac{d R}{d t}\right)^{2}+\frac{4 \mu}{\rho} \frac{d R}{d t}+\frac{2 \sigma}{\rho R}+\frac{P_{\infty}-P_{i}}{\rho}=0$ 
where $R$ is the bubble radius, $\mu, \sigma$ and $\rho$ are the viscosity, surface tension and density of the liquid, and $P_{\infty}$ and $P_{i}$ are the pressures in the liquid at infinity and in the bubble interior.

The bubble interior pressure can be approximated as:

$P_{i}=\left(P_{0}+\frac{2 \sigma}{R_{0}}\right)\left(\frac{R_{0}}{R}\right)^{3 \gamma} \quad$ (for an adiabatic collapse of a bubble)

$P_{i}=\left(P_{0}+\frac{2 \sigma}{R_{0}}\right)\left(\frac{R_{0}}{R}\right)^{3} \quad$ (for an isothermal collapse of a bubble)

where $P_{0}$ and $R_{0}$ are the equilibrium liquid pressure and initial radius of gas bubble in the liquid.

Equation 2.59 is mathematically very difficult to solve. However, some solutions have been obtained using a differential analyzer at the National Physical Laboratory. A numerical solution of this equation shows that the bubble radius increases to the maximum size and then falls with increasing rapidity, as shown in figure 2.13 [2.54]. However, the initial increase in bubble radius does not begin until Blake's threshold is reached. The maximum bubble radius can be considered to be achieved in two steps. In the first step, the bubble radius grows while the pressure difference across the bubble is 
negative and attains a size $R_{1}$. During the end of the first step, the liquid gains sufficient momentum and the bubble continues to grow even though the pressure across it is positive. At the end of the second step, the liquid kinetic energy is zero and the bubble radius is $R_{\max }$.

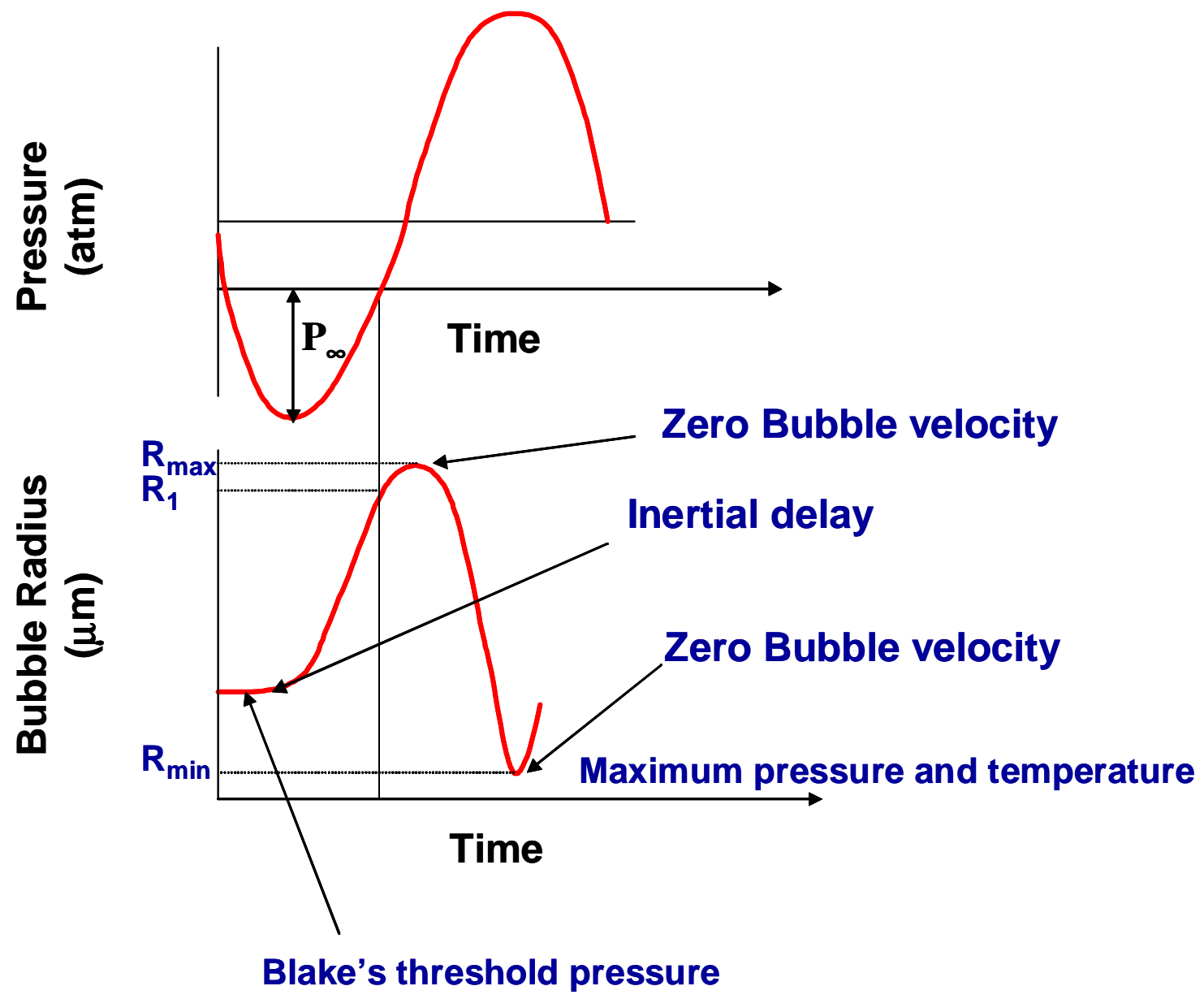

Figure 2.13: The change in size of a gas bubble during its growth and collapse [2.55] 


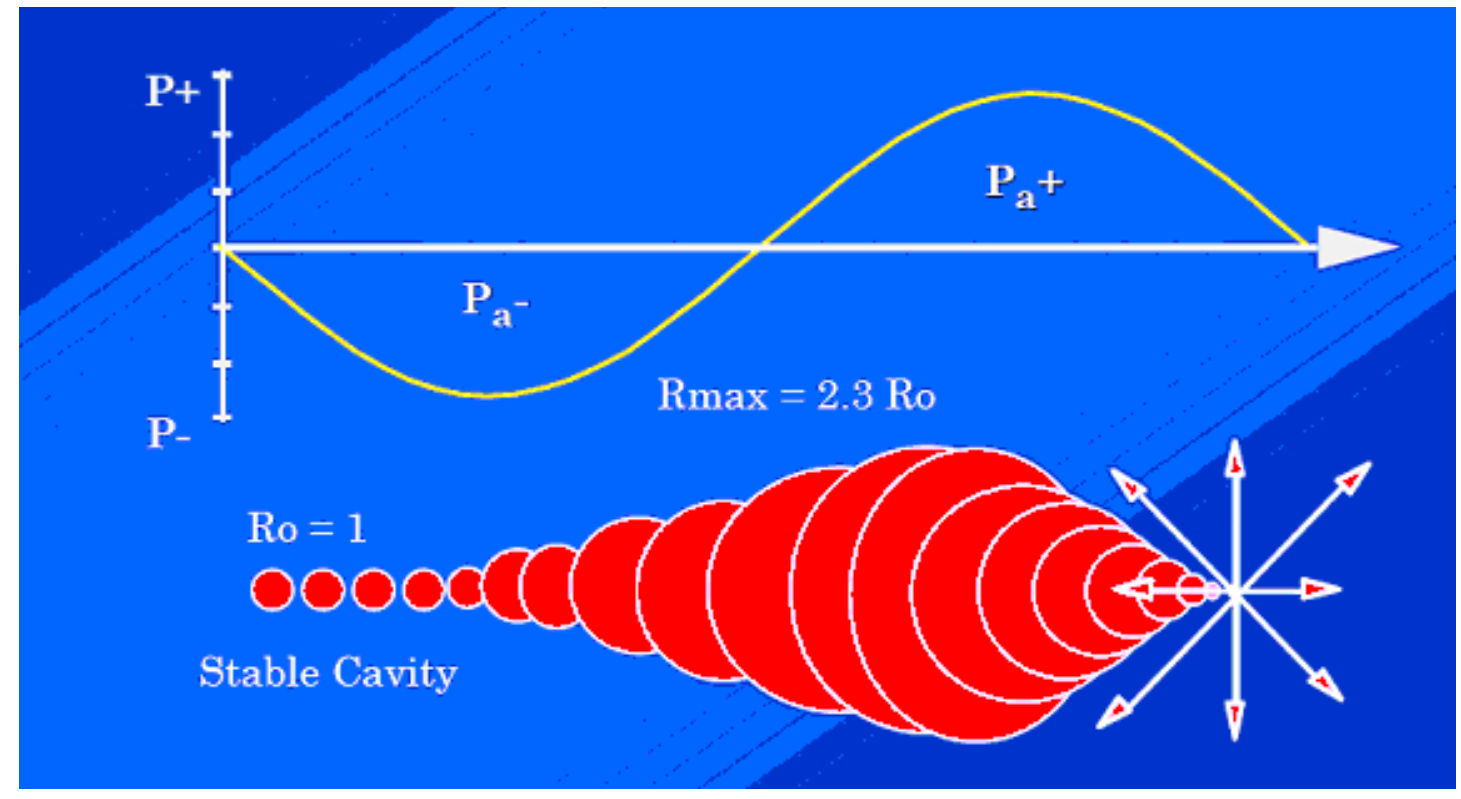

Figure 2.14: Schematic of bubble collapse [1.41]

During the collapse of the bubble, the surface tension forces become insignificant compared to the two opposing pressure terms and hence may be ignored [2.54]. It can be assumed that the collapse begins with the initial radius of the bubble $R_{\max }$, when the bubble is at its maximum size and ends when it reaches the minimum size $\left(R_{\min }\right)$. In the bubble equation when $R_{0}$ is replaced by $R_{\max }, P_{0}$ is the bubble pressure at the maximum bubble size and is therefore no longer 1 atm but much smaller. Ignoring the viscous forces, the bubble equation becomes:

$R \frac{d^{2} R}{d t^{2}}+\frac{3}{2}\left(\frac{d R}{d t}\right)^{2}+\frac{P_{\infty}-P_{0}\left(\frac{R_{\max }}{R}\right)^{3 \gamma}}{\rho}=0$ 
Noltingk et al. solved the above equation by considering that the bubble has zero velocity at its maximum and minimum size and reaches maximum velocity at zero acceleration. This allowed them to obtain equations for maximum bubble velocity during collapse (equation 2.63) and maximum pressure (equation 2.64) and temperature (equation 2.65) inside the bubble at the end of the collapse.

$\left(\frac{d R}{d t}\right)_{\max }^{2}=\frac{2 P_{\infty}(\gamma-1)}{3 \rho \gamma}\left[\frac{P_{\infty}(\gamma-1)}{P_{0} \gamma}\right]^{1 /(\gamma-1)}$

$P_{\max }=P_{0}\left[\frac{P_{\infty}(\gamma-1)}{P_{0}}\right]^{\frac{\gamma}{(\gamma-1)}}$

$T_{\max }=T_{0}\left[\frac{P_{\infty}(\gamma-1)}{P_{0}}\right]$ or $T_{\max }=T_{0}\left[\frac{R_{\min }}{R_{0}}\right]^{3 /(1-\gamma)}$

The $R_{\max }$ and $R_{\min }$ were correlated by computing the work done by the medium in compressing the bubble from its maximum to minimum size [2.55].

$$
\left(\frac{R_{\min }}{R_{0}}\right)^{\frac{3}{(1-\gamma)}}=(\gamma-1)\left(\frac{R_{\max }}{R_{0}}\right)^{3}
$$


Thus, knowing $R_{\max }$, one can estimate the maximum temperature reached during collapse of the bubble. This makes $R_{\max }$ a critical parameter in determining the final collapse conditions inside the bubble. It is important to know its dependence on primary variables such as initial radius of bubble $\left(R_{0}\right)$, acoustically applied pressure $\left(P_{\infty}\right)$, and frequency of acoustic waves $(w)$. As evident from figure 2.15, the maximum bubble radius increases with the initial bubble radius and source pressure amplitude but decreases with source frequency.
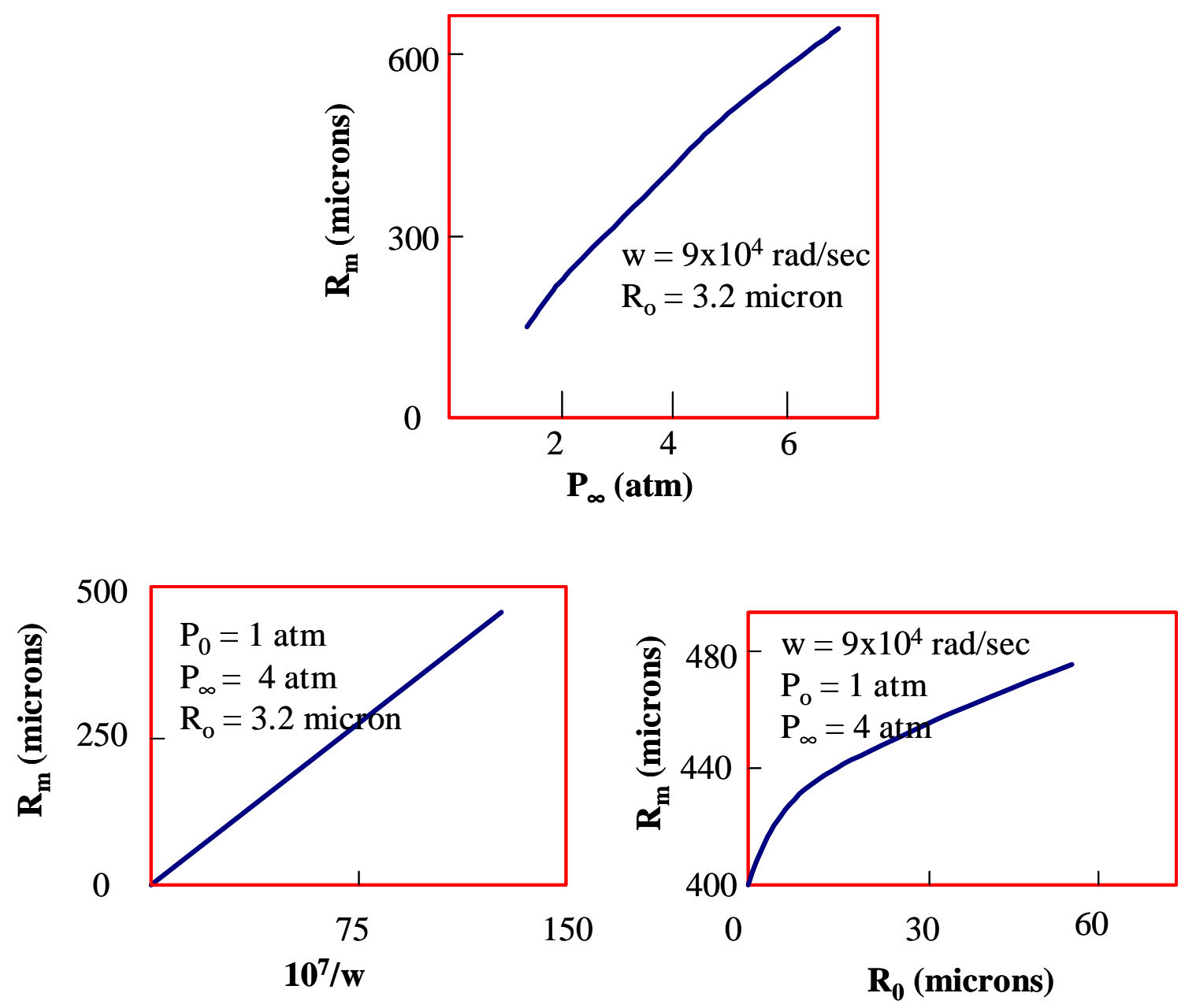

Figure 2.15: The variation of maximum bubble size with externally applied pressure, initial radius of bubble and frequency of acoustic wave [2.54] 
Apfel included the viscosity effect $(\mu)$ and obtained an analytical expression for the maximum radius of the bubble as a function of $R_{0}, P_{\infty}$ and $w$ [2.55]. In order to grow from its initial to the maximum size, the bubble has to pass through four time scales. The first one involves the time required for the bubble to cross Blake's threshold during which no change in bubble radius occurs. The second one is the delay caused due to the inertia and the viscosity of the liquid. In the next time interval, the bubble actually grows to a radius $\mathrm{R}_{1}$ due to the negative pressure difference across it. This negative pressure difference provides a momentum to the liquid surrounding the bubble which further increases the radius of the bubble to its maximum size, in spite of a positive pressure difference across the bubble.

$$
R_{\max }=\left[R_{0}+\frac{2}{3} \sqrt{\frac{P_{0}\left(\frac{P_{\infty}}{P_{0}}-1\right)}{\rho}} \tau\right]\left[1+\frac{2}{3}\left(\frac{P_{\infty}}{P_{0}}-1\right)\right]^{1 / 3}
$$

where

$$
\tau=\frac{1}{w}\left[\sqrt{\frac{2\left(\frac{P_{\infty}}{P_{0}}-1\right)}{\frac{P_{\infty}}{P_{0}}}}+\sqrt{2\left(1-\frac{P_{B}}{P_{\infty}}\right)}\right]-\frac{2}{3} R_{0} \sqrt{\frac{\rho}{\frac{2}{3} P_{0}\left(\frac{P_{\infty}}{P_{0}}-1\right)}}-\frac{8 \mu}{3 P_{0}\left(\frac{P_{\infty}}{P_{0}}-1\right)}
$$


Neppiras and Lush suggested that the maximum radius of a bubble reaches at least two times its initial size for transient cavities [2.48,2.50\&2.56]. The initial radius of bubble can be taken approximately as an average of $R_{\max }$ and $R_{\min }$. For a transient collapse, $R_{\min }=0$ and therefore $R_{\max }=2 R_{0}$. This allows one to use equation 2.68 to obtain the threshold pressure condition for transient cavitation by substituting $R_{\max }=2 R_{0}$ and $P_{\infty}=P_{T}$, where $R_{0}$ now becomes the threshold radius $R_{T}$ and $P_{T}$ is the threshold acoustic pressure for transient cavitation.

Neppiras has made a series of cavitation prediction charts showing Blake's threshold (lower transient cavitation threshold), the stable threshold for rectified diffusion and the transient cavitation threshold [2.57]. One such chart for air saturated water at an initial pressure of 1 bar and a source frequency of $20 \mathrm{KHz}$ is shown in figure 2.16. Curve $\mathrm{AB}$ corresponds to the Blake's threshold, $\mathrm{CD}$ to the transient threshold and EFG to the stable threshold for rectified diffusion. Any bubble below the Stable threshold (e.g. bubble at point $\mathrm{V}$ ) is in the dead zone and will either dissolve away or become stable at some small radius. A bubble, say the one at $\mathrm{X}$, in the region above the Stable threshold will grow by rectified diffusion and reach Blake's threshold where it will become unstable and eventually hit the transient threshold curve. The bubbles reaching the transient threshold curve expand, implode and disintegrate into small bubbles. These bubbles can again grow and repeat the so called transient cavitation cycle. Similarly, the bubble at point $\mathrm{W}$, which has already crossed Blake's threshold, grows by rectified diffusion and finally implodes as a transient cavity. The bubble exhibits an interesting 
behavior bubble at Y. This bubble will never reach transient threshold. It will grow by rectified diffusion and become large enough to separate out under gravity. This process is known as degassing. If the source frequency is not too low, the bubble may also reach its resonance size $\left(R_{r}\right)$. If the acoustic field is intense, the bubble will oscillate at very large amplitudes and throw microbubbles from their crest. One may also notice the steep stable threshold curve as the bubble approaches its resonance frequency. The bubble will require only a small pressure threshold at its resonance size to grow by rectified diffusion. As the bubble moves away from its resonance size, the threshold increases rapidly. Thus, these threshold curves provide a good source of information on the behavior of bubbles of different sizes in a known liquid irradiated with a sound field of specified pressure amplitude and frequency. 


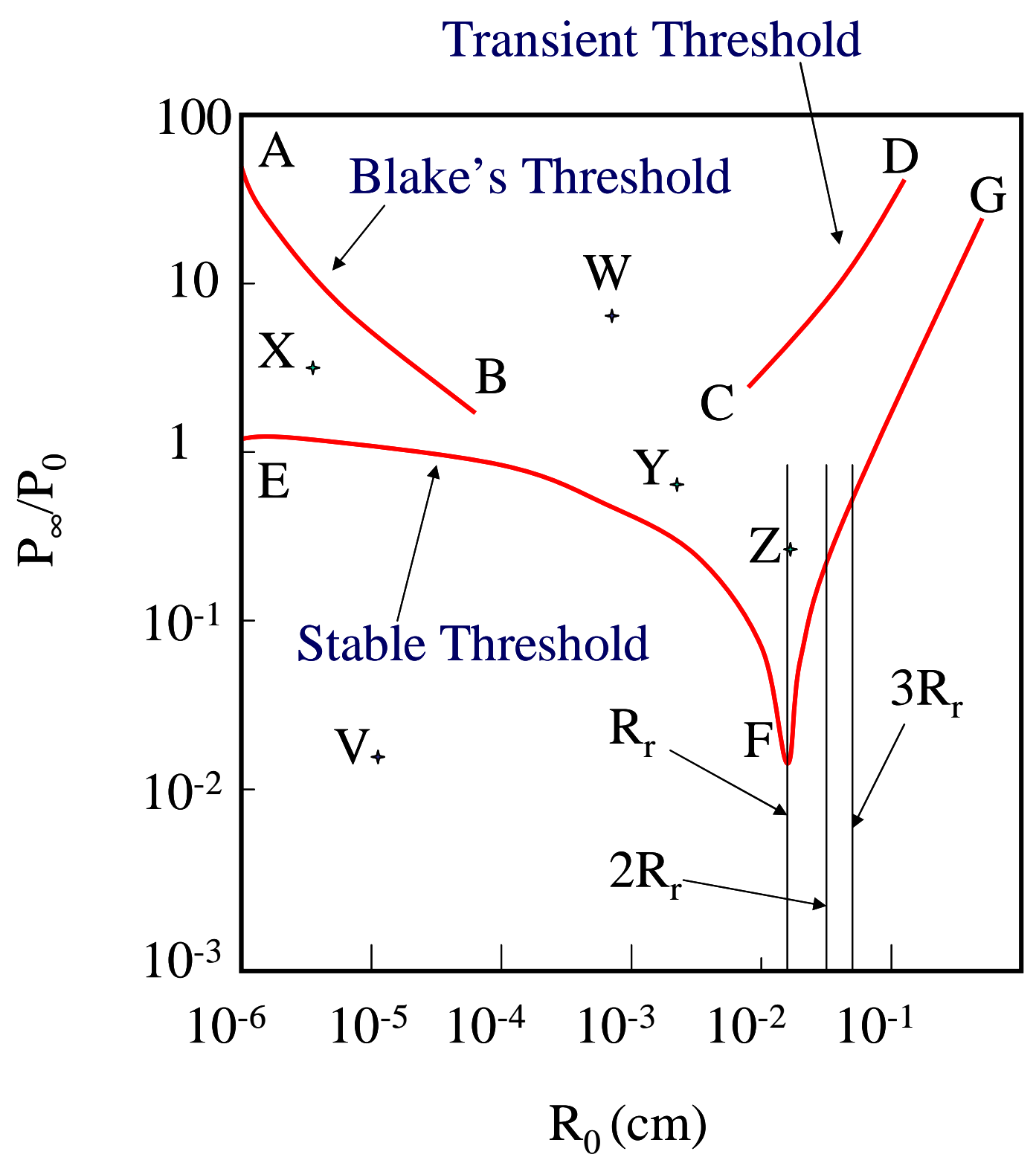

Figure 2.16: Blake's threshold, stable threshold and transient threshold for air saturated water at $20 \mathrm{KHz}$ [2.57] 


\subsubsection{Shock Wave and Fluid Jet Formation}

Bubbles driven into activity by acoustic waves act as sources of pressure variation and fluid motion [2.44]. For example, bubbles driven at a resonance frequency entail high fluid velocity causing enhanced heat, mass and momentum transfer. These high fluid velocities occur at cavitation sites, the most common forms of which include contaminants and impurities on the surfaces. This situation is very advantageous for removal of particulate or other contaminants from the surfaces. Whether it be large scale cleaning using ultrasonics or cleaning of fine structures using megasonics, the type of cavitation must produce high velocities for creating necessary drag forces on the particles without powerful inertial cavitation which might cause damage to the features on the surface or might also pit the surface. To lower the destructive effect of cavitation, a solvent with higher vapor pressure can be added which provides a cushioning effect to the collapsing cavity and softens its impact. Similarly, addition of gas to the liquid reduces the impact of cavitation.

Ohl, et al., have shown that the dynamics of bubble collapse depend on the distance of separation between the solid boundary and the bubble center [2.58]. When this distance is three times or greater the radius of the bubble, the bubble stays spherical during its collapse and shock waves are emitted [2.59]. If the distance is lower than three times the bubble radius, asymmetrical collapse of the bubble occurs resulting in a fluid jet formation. Shock waves are emitted in this case also, but are less violent. 
Flynn suggests that during transient cavitation, the liquid around the liquid implodes and rebounds with extreme pressures [2.50]. We know that the speed of sound is an increasing function of liquid pressure. This leads to a significant increase in sound speed during collapse. The waves start to crowd one another and this is just the condition required for a shock wave to form. Benjamin reported a correlation between maximum gas pressure $\left(P_{\max }\right)$ inside the collapsing bubble and the radial distance $(r)$ at which shock will form [2.48].

$$
P_{\text {max }} \approx 13.6\left(\log \frac{r}{R_{\text {min }}}\right)^{-2 / 3}
$$

Minsier et al. illustrated that a physical discontinuity occurs between the liquid pressure and velocity during bubble collapse, suggesting the presence of a shock wave [2.60]. The Gilmore model was used in their numerical computations. It was demonstrated that rebound of the liquid after bubble collapse results in spikes in liquid velocity at few radial distances from the bubble center. These spikes correspond to the liquid velocity at the shock wave front as shown in figure 2.17 . One can notice the decrease in spikes or shock wave front velocity over time. 


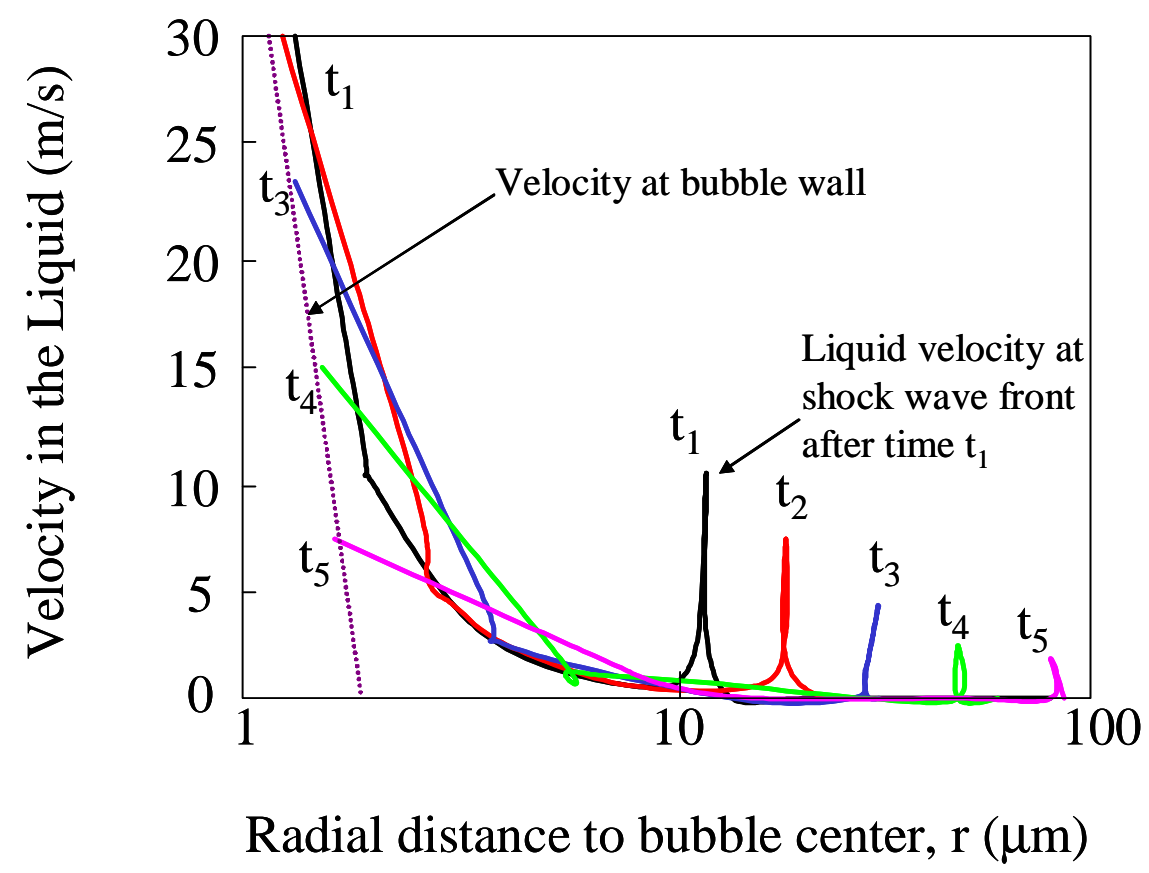

Figure 2.17: Liquid velocity as a function of distance from bubble center after specified elapsed time [2.60]

The effect of solution parameters on liquid velocity at the shock wave front is critical in cavitation induced cleaning of surfaces. A high shock wave velocity can clean but also damage the surface. Therefore a need to adjust the solution conditions to optimize the velocity of liquid at shock wave front is necessary. Cavitation process variables such as initial bubble radius, sound source pressure amplitude and solution surface tension were investigated for their effect on liquid velocity at the shock wave front. The calculations were performed for water and results are displayed in figures 2.18 and 2.19. It was observed that the liquid velocity at the shock front increases with acoustic pressure and goes through a maximum as a function of the initial radius of the 
bubble. The trend is similar to that observed for maximum velocity at the bubble wall, the latter values being much higher in magnitude (of the order of 1000-6000 m/s depending on the solution conditions). In the case of effect of surface tension, the liquid velocity at the shock front decreases with surface tension. This was attributed to the fact that the maximum bubble velocity decreases with an increase in surface tension due to the lower maximum radius of the bubble attained during expansion in liquid with higher surface tension.

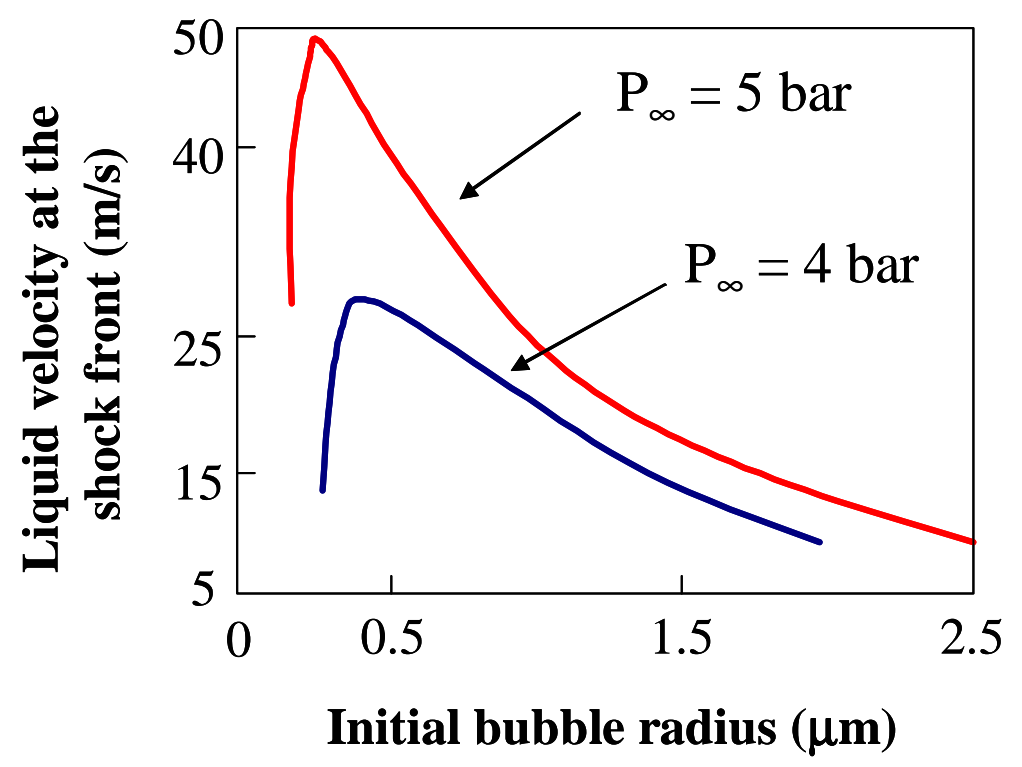

Figure 2.18: Effect of source pressure amplitude and initial bubble radius on liquid velocity at shock wave front [2.60] 


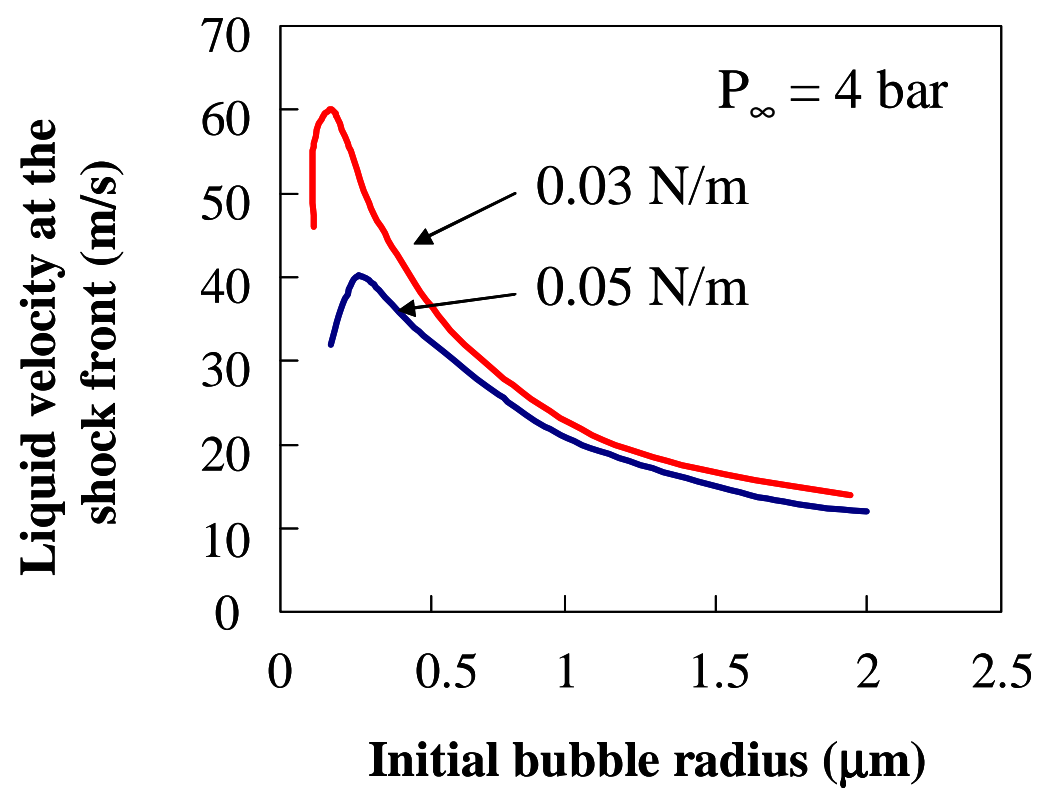

Figure 2.19: Effect of liquid surface tension and bubble radius on liquid velocity at shock wave front [2.60]

Plesset (1966) had indicated that the stresses produced by rebound during collapse of a spherical bubble fall off rapidly with distance and hence may not have a significant impact on damage of surfaces observed during cleaning. Kornfeld and Suvorov proposed that liquid jets are formed during bubble collapse close to a solid boundary. These bubbles start their collapse as spheres followed by elongation in the direction normal to the wall and then form an inward moving jet on the side of the bubble opposite the wall.

Plesset, et al., used a numerical method for collapse of vaporous bubbles lacking spherical symmetry and obtained the jet velocities for two cases: a bubble initially in contact with a solid boundary and a bubble initially half its radius from the boundary at 
the nearest point [2.61]. The jet velocities of $130 \mathrm{~m} / \mathrm{s}$ and $170 \mathrm{~m} / \mathrm{s}$ were obtained respectively for the two cases. The shapes of the bubbles and jet formations during the collapse for the two cases are shown in figures 2.20 and 2.21.

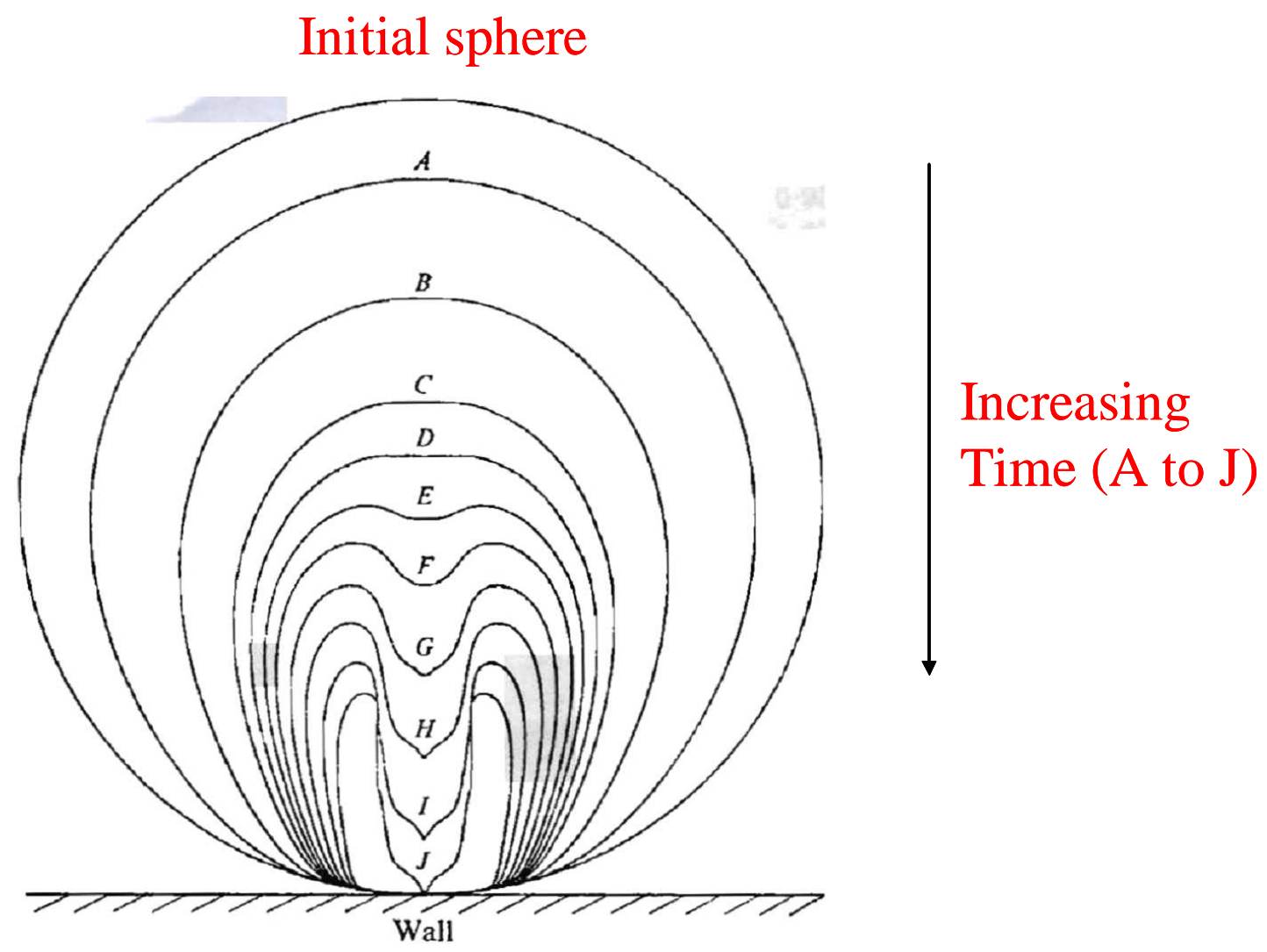

Figure 2.20: Collapse of a spherical bubble initially in contact with solid boundary [2.61] 

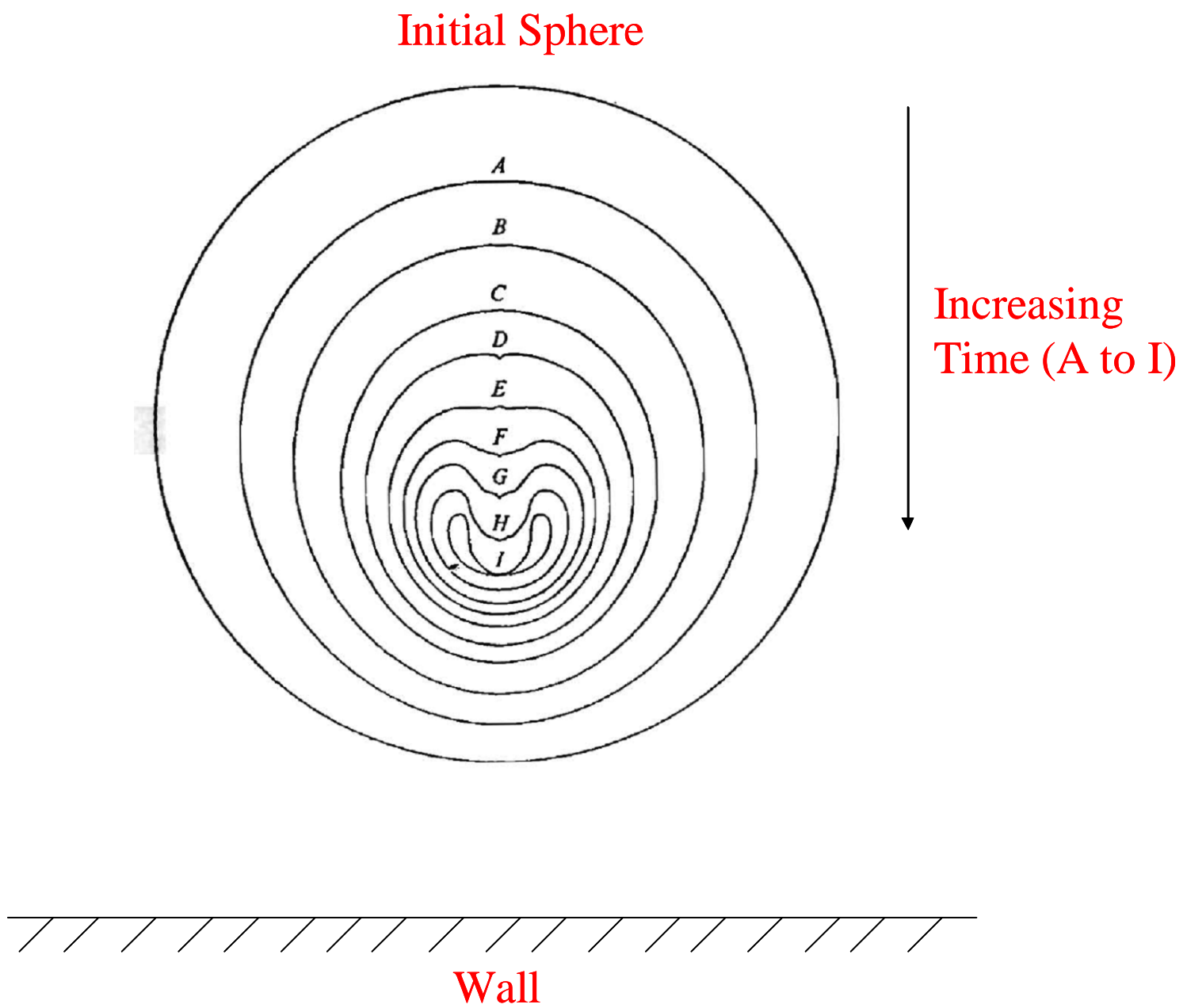

Figure 2.21: Collapse of a spherical bubble initially in contact with solid boundary [2.61]

The cavitation effect in the liquid can be measured using a phenomenon known as sonoluminescence, which is the name given to the light emitted when a liquid is cavitated in a specified manner. Sonoluminescence is always preceded by cavitation and gives a good account of prevailing conditions inside the cavitating bubble. The effect was first 
discovered by Marinesco and Trillat in 1933 on a photographic plate using high intensity ultrasonic field. The following section gives a brief review of sonoluminescence.

\subsubsection{Sonoluminescence}

Sonoluminescence (SL) is described mainly as recombination of free radicals formed in an extreme high temperature and pressure environment during the collapse of a transient cavity or in a highly non linear but stable cavitation [2.62]. However, since the compression ratio (ratio of maximum to minimum bubble radius) and rate of compression $(d R / d t)$ are lower for stable cavities, they must have lower temperatures than transient cavities. Thus the cavitation effect produced by stable bubbles is milder than that of transient cavities. The sonoluminescence flashes lie in the ultraviolet range of light spectra and are usually light blue in color. The flashes are quite weak and photomultiplier tubes are commonly used to detect them. SL is measured or experimentally represented as sonoluminescence counts, phot, photons count rate, luminous intensity, sonoluminescence flux or intensity, light intensity, and others.

There are two main types of SL: single bubble sonoluminescence (SBSL) and multi bubble sonoluminescence (MBSL). SBSL refers to SL from a single stable bubble held in liquid irradiated with acoustic waves, whereas MBSL is light emission from a large number of interacting bubbles that last only for a few acoustic cycles. The various theories that have been proposed to explain this light emission phenomenon include 
Bremsstrahlung radiation from hot plasma core, black body radiation, electrical mechanisms such as fractoluminescence, the Cashmier effect, radiation from quantum tunneling and collision-induced emission [2.63]. These theories can be broadly classified into electrical and thermal hypothesis [2.64]. The electrical hypothesis proposes that rapid compression of bubble causes an outflow of electrons leading to microdischarge due to the excitation and ionization of gas species adsorbed on the bubble interface. The thermal hypothesis predicts the formation of excited and reactive species in a hot environment inside the bubble due to its adiabatic collapse.

At high temperatures, electronic excitations are the most probable means of emissions. The excitation of water into its triplet state in the presence of a dissolved gas molecule can result in near UV emission with a peak maximum at $270-290 \mathrm{~nm}$. The excited water molecule may also fragment into $\mathrm{H}$ and an electronically excited $\mathrm{OH}$ radical that on relaxation back to ground state shows emission at $310 \mathrm{~nm}$ [2.64].

$$
\begin{aligned}
& \left.\mathrm{H}_{2} \mathrm{O}+\mathrm{M} \stackrel{\text { acoustic wave }}{\longrightarrow} \mathrm{H}_{2} \mathrm{O}+\mathrm{M} \text { (hot molecules }\right) \longrightarrow \mathrm{H}_{2} \mathrm{O} *\left({ }^{3} \mathrm{~B}_{1}\right)+\mathrm{M} \\
& \mathrm{H}_{2} \mathrm{O} *\left({ }^{3} \mathrm{~B}_{1}\right)+\mathrm{M} \longrightarrow \mathrm{H}_{2} \mathrm{O}\left({ }^{1} \mathrm{~A}_{1}\right)+h v_{0}(270-290 \mathrm{~nm}) \\
& \mathrm{H}_{2} \mathrm{O}^{*}\left({ }^{3} \mathrm{~B}_{1}\right)+\mathrm{M} \longrightarrow \mathrm{H}+\mathrm{OH} * \mathrm{OH}+\mathrm{H}+h v_{1}(310 \mathrm{~nm})
\end{aligned}
$$


Griffing, et al., suggested the formation of a quasimolecule $\mathrm{H}_{2} \mathrm{O}$ stabilized by a radiative process for emissions around $400 \mathrm{~nm}[2.65,2.66]$. Saxena and Nyborg hypothesize that a third body M, such as a gas or water molecule, forms a weak bonded structure $\mathrm{M}-\mathrm{OH}$ which combines with $\mathrm{H}$ to give a quasimolecule $\mathrm{H}-\mathrm{M}-\mathrm{OH}$ that releases water and $\mathrm{M}$ by radiative or non radiative process with emission of photons [2.67].

$\mathrm{H}+\mathrm{OH} \longrightarrow \mathrm{H} \cdots \mathrm{O} \cdots \mathrm{H} \longrightarrow \mathrm{H}_{2} \mathrm{O}+h v_{2}$

$$
\mathrm{M}+\mathrm{OH} \longrightarrow \mathrm{M} \cdots \mathrm{OH} \longrightarrow \mathrm{H}-\mathrm{M}-\mathrm{OH} \longrightarrow \mathrm{H}_{2} \mathrm{O}+\mathrm{M}+h \mathrm{v}_{2}
$$

In the case of hydrocarbons such as benzene or aliphatic alcohols, $\mathrm{OH}$ and $\mathrm{H}_{2} \mathrm{O}$ radicals combine with hydrocarbons which results in a linear decrease of total emission [2.64].

$\mathrm{OH}^{*}+\mathrm{RH} \longrightarrow$ products

$\mathrm{H}_{2} \mathrm{O} *+\mathrm{RH}$ products

Flint and Suslick were the pioneers in measuring the multibubble SL spectra from hydrocarbon and hydrocarbon liquids [2.68]. Didenko, et al., used $\mathrm{C}_{2}$ emission from a water- benzene mixture to estimate the temperature of excitation following the method of 
Flint and Suslick. They concluded that an emission vibrational temperature of $4300 \pm 200$ K exists inside the bubble during cavitation in water [2.69].

The SL intensity depends on the temperature and pressure conditions inside of the cavitating bubbles and is therefore related to the nature of the solvent, dissolved gas, liquid temperature, frequency of sound, hydrostatic pressure of the liquid, and source pressure amplitude.

The dependence of SL on hydrostatic pressure and sound pressure amplitude comes from the dependence of the maximum temperature attained by the gas in the bubble during the final phases of collapse on $\left(\mathrm{P}_{\infty}-\mathrm{P}_{0}\right)$ and $\left(\mathrm{P}_{\infty}+\mathrm{P}_{0}\right)$. Although, the velocity of the collapse of bubble wall increases with $\mathrm{P}_{0}$, the maximum radius attained decreases with $\mathrm{P}_{0}$. The net effect is a maximum in SL intensity when the two opposing tendencies counterbalance. In the case of $\mathrm{P}_{\infty}, \mathrm{SL}$ increases with an increase in sound wave pressure amplitude. Walton and Reynolds (1984) provide a very good review on experimental studies of the effect of $\mathrm{P}_{\infty}$ and $\mathrm{P}_{0}$ on SL.

Griffing and Sette (1955) were first to study the effect of sound frequency on SL. They found that SL decreased from $2 \times 10^{-8}$ phot at $660 \mathrm{~Hz}$ to $10^{-8}$ phot at $1 \mathrm{MHz}$ and to $3 \times 10^{-9}$ phot at $2 \mathrm{MHz}$ in tap water at a sound intensity of $2 \mathrm{~W} / \mathrm{cm}^{2}$. The higher the frequency, the shorter is the period of bubble expansion and the milder is the bubble collapse. 
The effect of liquid temperature on SL is very complex. The variation in liquid temperature can affect several properties of liquids such as viscosity, surface tension, vapor pressure and dissolved gas content. Hence it is not easy to relate the effect of liquid temperature on SL. Jarman (1959) investigated 15 liquids with widely differing properties and found a correlation between SL and the ratio of the square of surface tension and vapor pressure of the liquid. The higher the ratio, the greater was the SL flux. The increase in vapor pressure with temperature reduces the speed of the collapsing bubble wall which can cause the collapse to be less violent. The amount of dissolved gas in a liquid increases with a decrease in temperature. The higher amount of gas cushions the impact of a collapsing bubble and reduces the SL.

The role of dissolved gases on SL is also attributed to the fact that gases have different thermal conductivities $(\mathrm{k})$ and ratio of heat capacities at constant pressure and volume $(\gamma)$. If the bubbles are sufficiently small, heat conduction becomes important for adiabatic collapse of the bubble. Thus SL intensity will depend on k of dissolved gas as long as time for heat conduction is available (sound frequency is not too high). In an experimental study by Young where SL was measured for rare gases dissolved in water, a strong dependence was observed between SL and thermal conductivity of rare gas and SL intensity. Xenon with a lower thermal conductivity of $0.1 \times 10^{-4} \mathrm{cal} \mathrm{cm}^{-1} \mathrm{~s}^{-1} \mathrm{C}^{-1}$ exhibited SL of $125 \times 10^{10}$ phots whereas helium with higher thermal conductivity of 3.8 x $10^{-4} \mathrm{cal} \mathrm{cm}^{-1} \mathrm{~s}^{-10} \mathrm{C}^{-1}$ showed lower SL of $1.16 \times 10^{10}$ phots. 
Thus, sonoluminescence has shown great potential for determination of actual conditions inside a bubble during cavitation and serves as an important guide for important applications of bubble dynamics and cavitation such as sonochemistry, ultrasonic and megasonic cleaning, cavitation damage and ultrasound diagnostics.

\subsubsection{Acoustic Streaming}

It is a well known fact that particle velocities in an acoustic field may not simply be sinusoidal even though the motions of sound sources essentially are sinusoidal (Nyborg 1965). Patterns of steady vortices or time independent circulations of fluid were first observed by Faraday and Dvorak [2.70]. Rayleigh did the first theoretical analysis of such phenomena and deduced the flow patterns $[2.71,2.72]$. It was found that a time independent component of velocity exists in addition to the oscillating component which can be obtained by solving the Navier-Stokes equation governing the flow. This occurs because of small drifts in the position of fluid particles during each acoustic cycle, caused by an attenuation of the wave in a viscous medium [2.73]. These flows occur either in a non-uniform sound field or near a solid boundary immersed in fluid irradiated with sound field or near the oscillating sound source itself. The flow velocity increases with sound intensity but is always smaller than the fluid particle velocity in the sound wave [2.74]. 
There are three types of streaming that are classified according to scale namely Eckart streaming, Schlichting streaming and microstreaming.

\subsubsection{Eckart Streaming}

Eckart streaming is a free non-uniform sound field in which the inhomogeneity scale of the sound field is much larger than the acoustic wavelength. It is characterized by streaming patterns on the scale of volume of flow field. Nyborg outlined the fundamental equations that govern the phenomenon of acoustic streaming $[2.75,2.76]$. The Eckart streaming velocity is a time independent velocity that depends on the frequency of the sound wave, intensity of the transducers and the fluid properties. Figure 2.22 shows the schematic of a typical streaming velocity profile in a megasonic tank. Maximum velocity occurs at the center of the transducer and approaches zero close to the wall. In figure 2.23, the maximum streaming velocity, for water, is plotted as a function of acoustic frequency and transducer intensity. One may notice clearly that the effect of frequency is more pronounced than that of transducer intensity. The details on derivation of streaming velocity are included in Appendix A.1 for interested readers. 


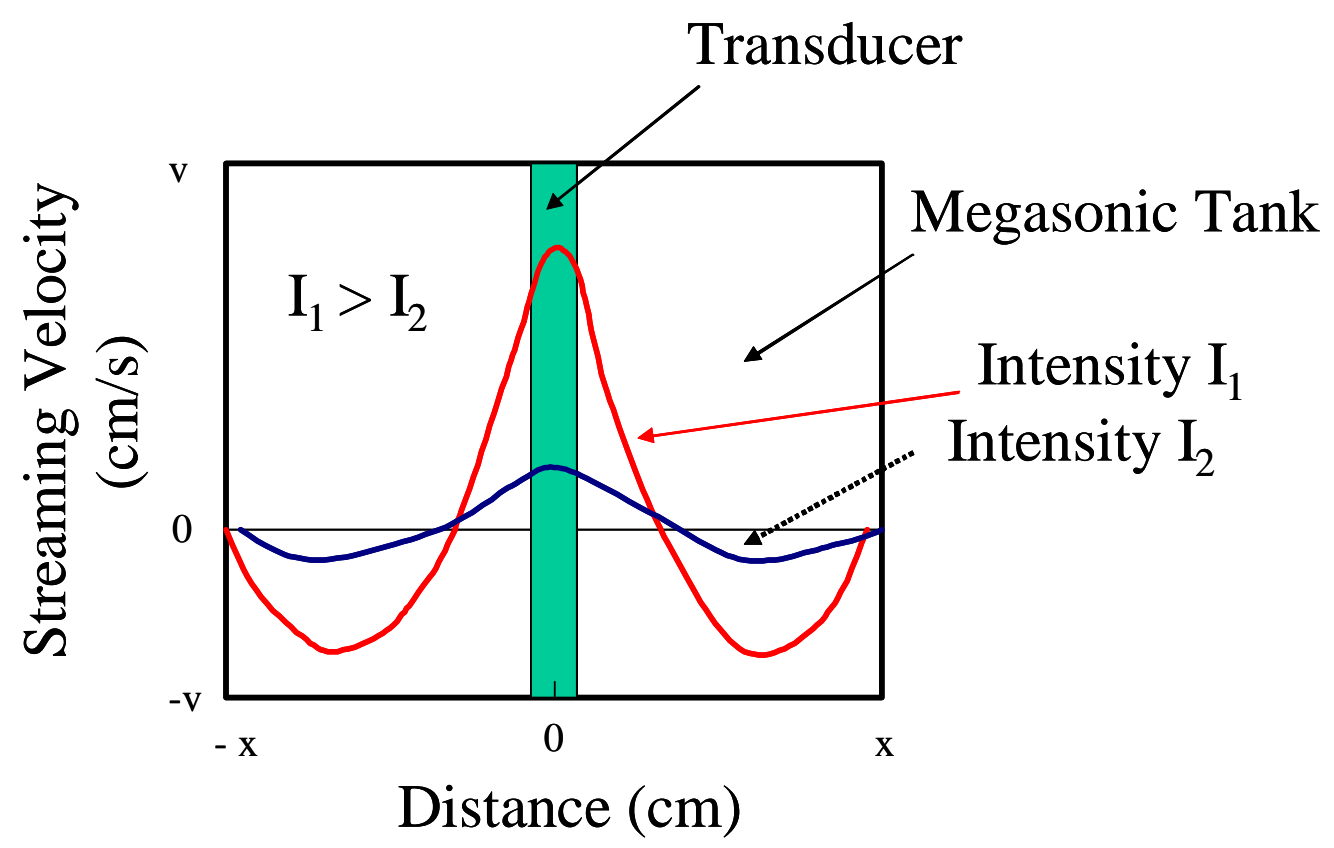

Figure 2.22: Schematic of Eckart streaming velocity profile in a megasonic cleaning tank

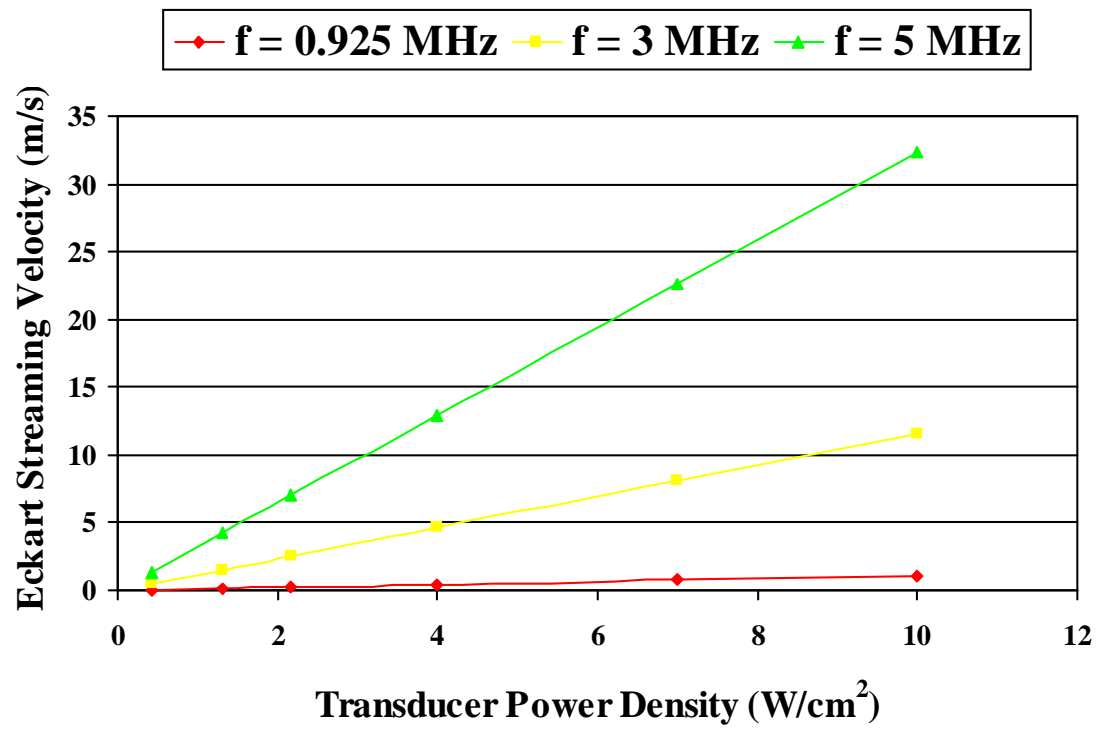

Figure 2.23: Maximum Eckart streaming velocity at different transducer intensities and sound wave frequency in a megasonic tank 


\subsubsection{Schlichting Streaming and Microstreaming}

Schlichting streaming is a vortex flow inside the viscous layer resulting from interactions with a solid boundary. The vortices extend for about twice the boundary layer thickness [2.77]. Steady viscous stresses are exerted on the boundaries where this type of rotational motion occurs, and these stresses may contribute significantly to removal from surface layers.

Acoustic microstreaming occurs due to scattering of the sound waves from the oscillating bubbles, especially resonating bubbles, generating rapid currents in localized regions. Vibration of bubbles close to the solid boundary shows several stages of streaming. A stable mode of bubble vibration is observed when bubble surface velocity is close to $11 \mathrm{~cm} / \mathrm{s}$ causing microstreaming near the top of the bubble [1.45]. A reversal in streaming occurs at a bubble surface velocity of $31 \mathrm{~cm} / \mathrm{s}$ followed by chaotic surface agitation at $60 \mathrm{~cm} / \mathrm{s}$. At this bubble surface velocity, transformation from stable mode occurs and the bubble is surrounded by a large vortex ring [1.45]. Microstreaming can be very useful in transporting the particles from the viscous boundary to the bulk of the solution.

Schematics of Schlichting streaming and microstreaming are shown in figures 2.24 and 2.25 respectively. 


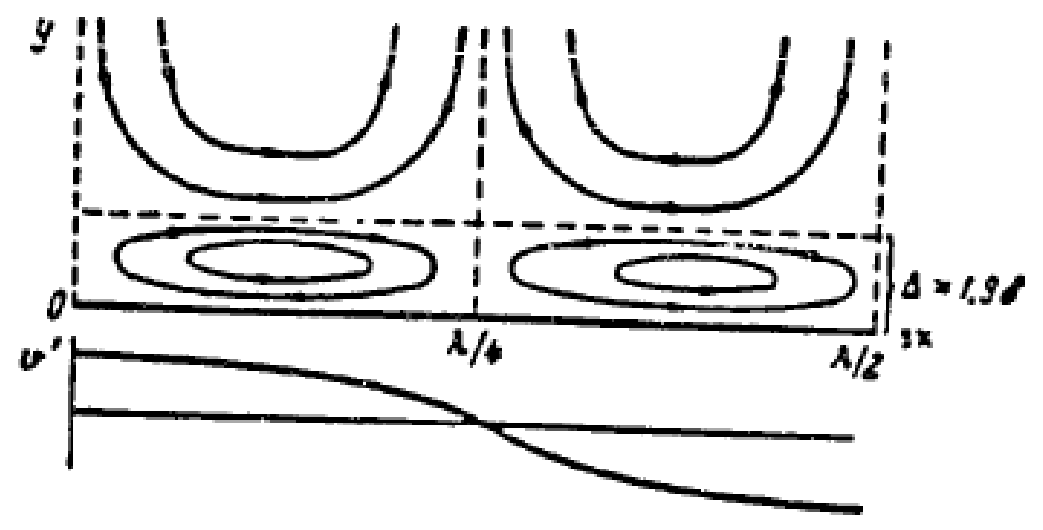

Figure 2.24: Schlichting streaming [2.76] 

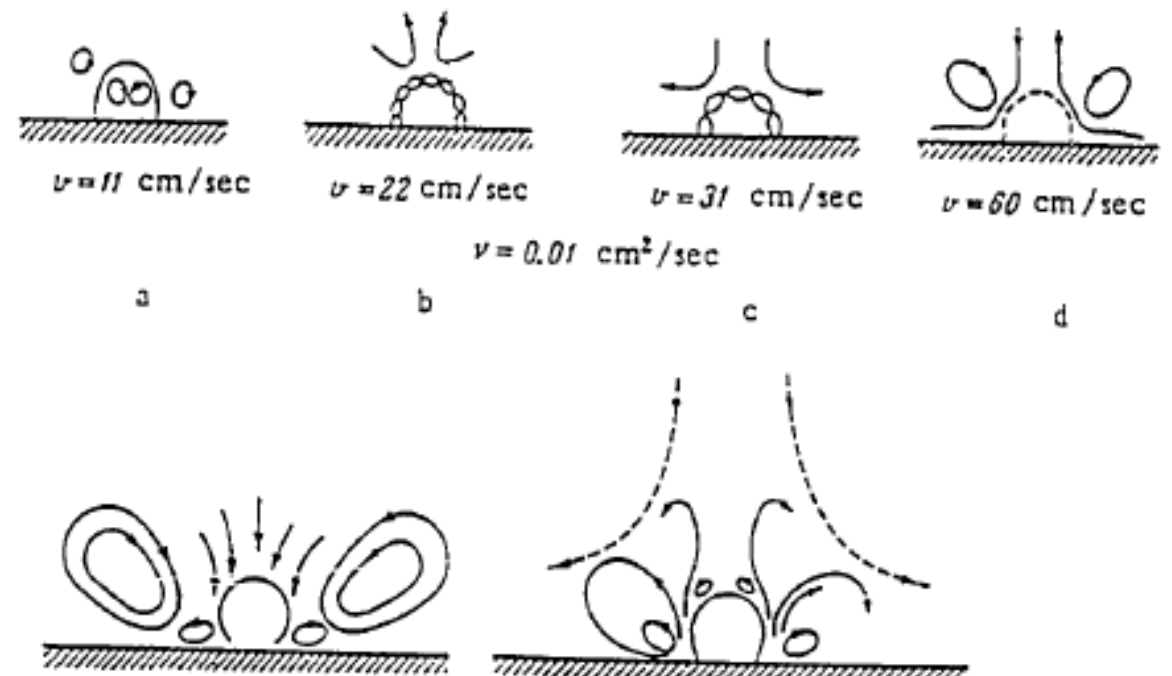

$\nu=0,40 \mathrm{~cm}^{2} / \mathrm{sec}$

$v=62 \mathrm{~cm} / \mathrm{sec}$

c
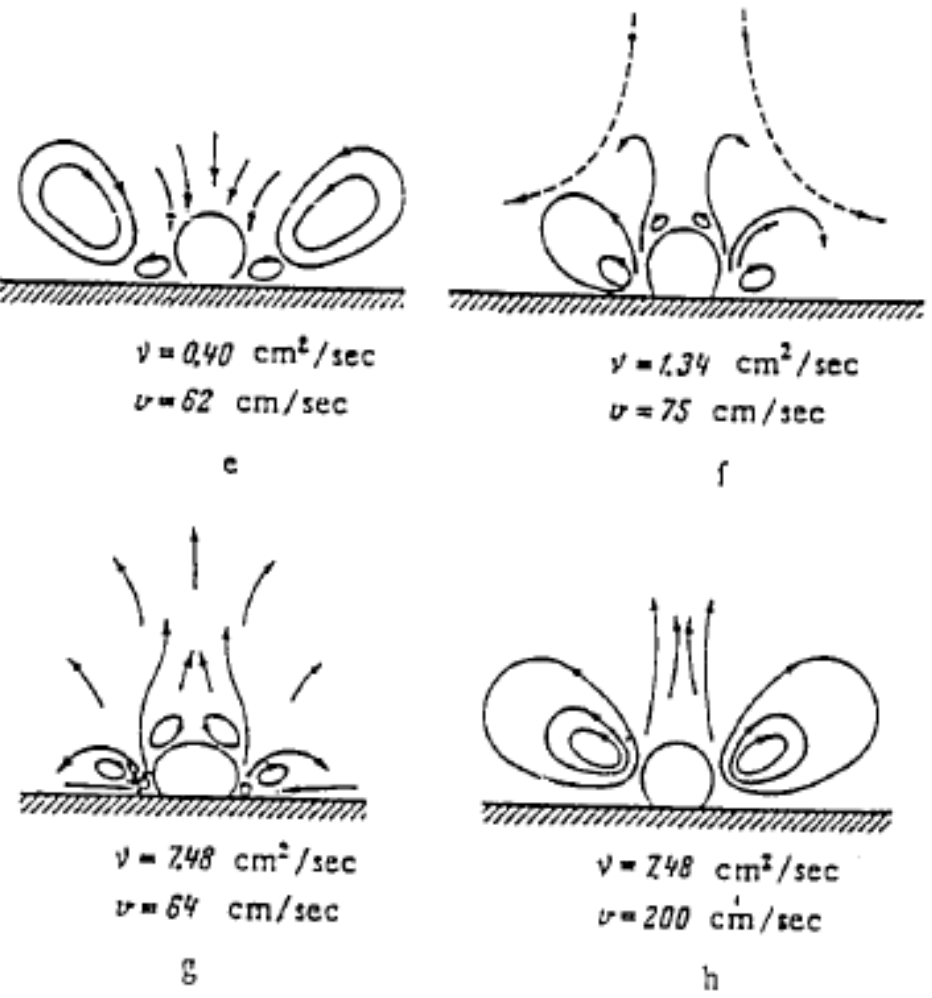

Figure 2.25: Sequence of microstreaming patterns at different bubble surface velocities [2.76] 


\section{CHAPTER 3: MATERIALS AND METHODS}

\subsection{Materials}

Silicon (100) wafers (150 mm, p-type, 11-18 $\Omega$-cm) were used for cleaning experiments conducted in a class-10 cleanroom. Aminated and plain silica particles (Corpuscular Inc.) with a mean size of $367 \pm 20 \mathrm{~nm}$ and $372 \pm 15 \mathrm{~nm}$ respectively were used as contaminating particles. The different chemicals used in various stages of this work include potassium chloride $(\mathrm{KCl})$, sodium chloride $(\mathrm{NaCl})$, lithium chloride $(\mathrm{LiCl})$, cesium chloride $(\mathrm{CsCl})$, hydrochloric acid $(\mathrm{HCl})$, ammonium hydroxide $\left(\mathrm{NH}_{4} \mathrm{OH}\right)$, sodium hydroxide $(\mathrm{NaOH})$, potassium hydroxide, and hydrogen peroxide $\left(\mathrm{H}_{2} \mathrm{O}_{2}\right)$. All the chemicals used were electronic grade with purity greater than $99 \%$.

\subsection{Megasonic System}

A poly-vinylidene fluoride (PVDF) tank, donated by ProSys Inc., $25.4 \mathrm{~cm}$ x 25.4 $\mathrm{cm} \times 29.8 \mathrm{~cm}$ in size, consisting of four rectangular transducers with a total area of 164 sq $\mathrm{cm}$ and an operating frequency of $925 \pm 25 \mathrm{KHz}$, was used for megasonic exposure of the wafers [3.1-3.3]. A control unit composed of three modules, namely a system controller (MegaPulse $\left.{ }^{\mathrm{TM}}\right)$, a power director $\left(\mathrm{MegaPlex}^{\mathrm{TM}}\right)$ and a megasonic power source (MegaPower ${ }^{\mathrm{TM}}$ ), was used to operate all the transducers simultaneously, resulting in a 
maximum power density of $2.17 \mathrm{~W} / \mathrm{cm}^{2}$. Figure 3.1 shows the details of transducer array bonding.

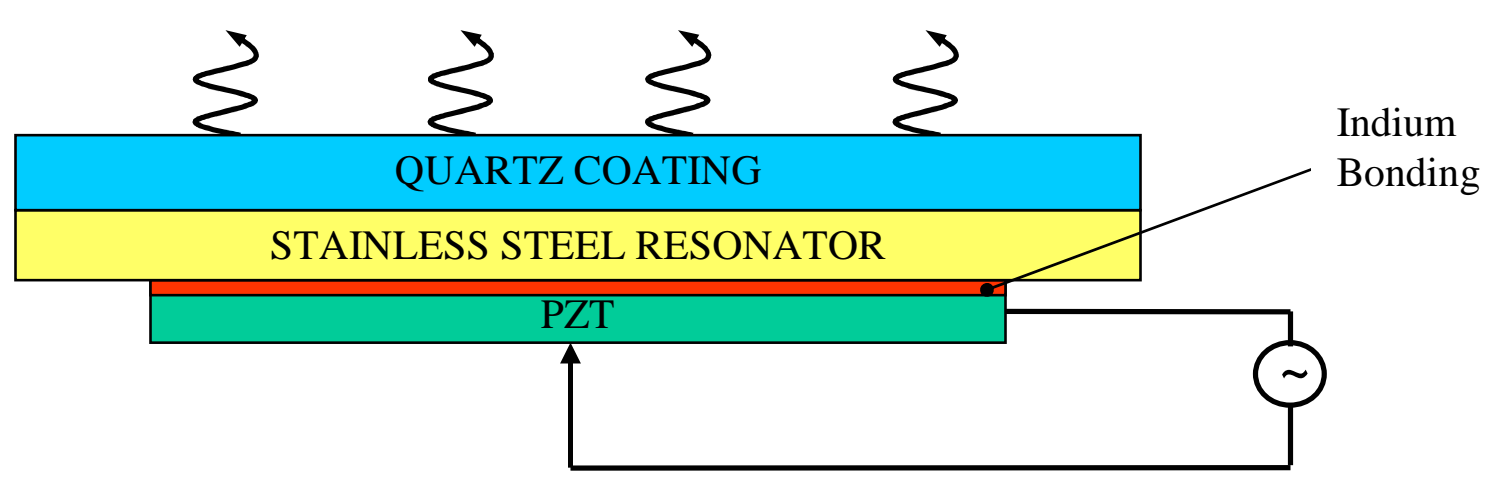

Figure 3.1: Schematic of transducer bonding details

The transducer array includes a piezoelectric crystal for generating acoustic energy, stainless steel resonators for positioning the piezoelectric crystal and a quartz cover for transmitting the energy to the fluid in the tank. This hydrophilic quartz cover is useful in reducing reflected acoustic powers for a wide range of liquids. The indium layer offers good bonding between stainless steel resonators and the piezoelectric crystal.

The cleanroom DI water (18 mega ohm-cm), used to prepare the experimental solutions was circulated through a $50 \mathrm{~nm}$ poly tetrafluoroethylene (PTFE) filter (Pall Corp.) using a double diaphragm type pump (Wilden Inc.). A schematic of the megasonic system is shown in figure 3.2. 

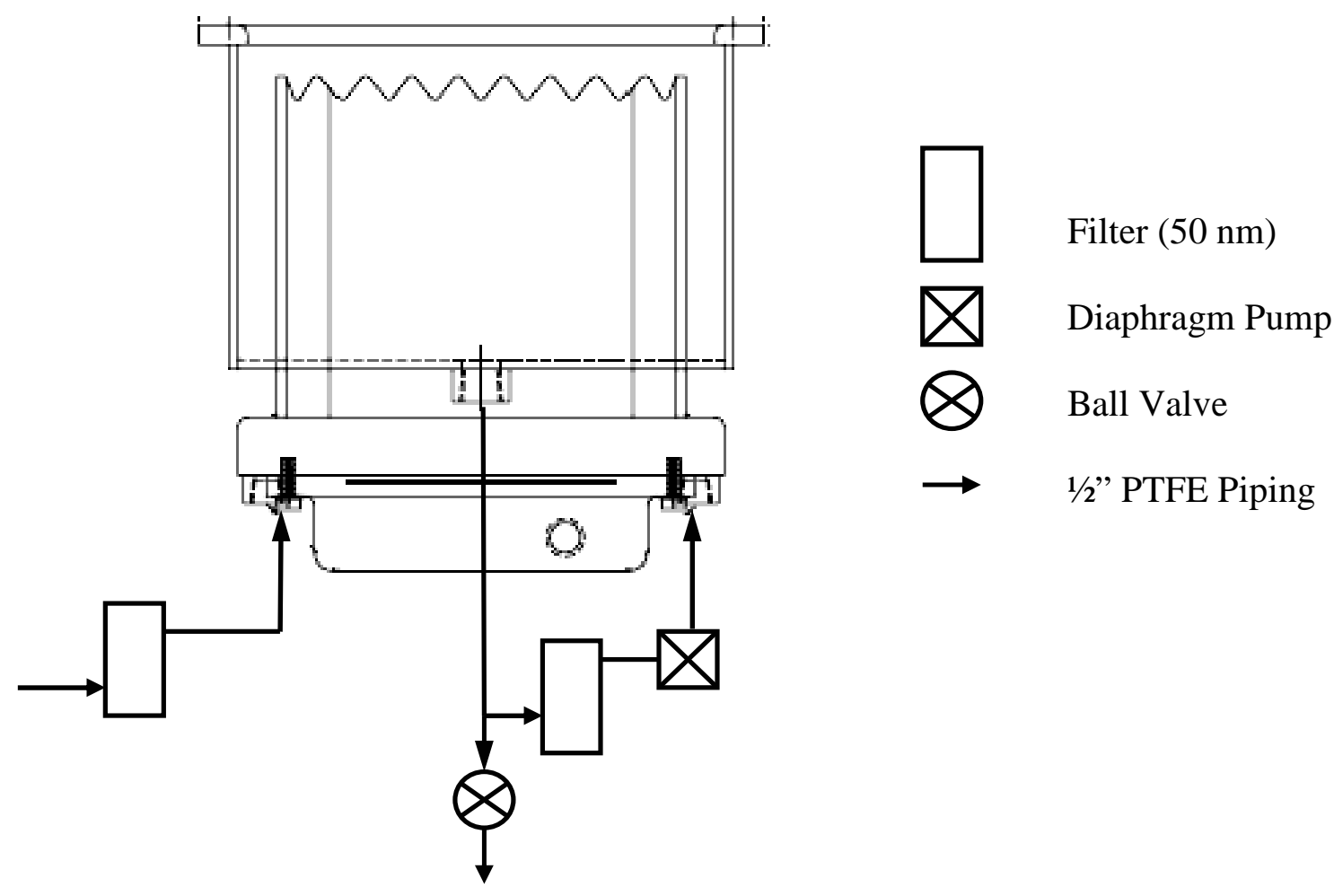

Figure 3.2: Schematic of megasonic system 


\subsection{Particle Size Measurements}

The size distribution of plain and aminated silica particles was obtained using two techniques, namely Field Emission Scanning Electron Microscopy (FE-SEM) and Photon Correlation Spectroscopy (PCS) using a Hitachi S-4500 and a Beckman Coulter N4 Plus Submicron Particle Sizer respectively. The PCS technique determines the particle size by measuring the rate of fluctuations in laser light $(10 \mathrm{~mW}$ helium-neon laser at $632.8 \mathrm{~nm})$ intensity scattered by particles as they diffuse through a fluid.

The FE-SEM images of aminated and plain silica particles are shown in Figure 3.3 and 3.4 respectively. The particles appear circular and uniform in size. The mean sizes of aminated and plain silica particles obtained using this technique were found to be $367 \pm$ $20 \mathrm{~nm}$ and $372 \pm 15 \mathrm{~nm}$, respectively. However, some agglomeration of particles was observed even though the dispersion containing these particles was sonicated at $40 \mathrm{KHz}$, using Branson 5510, for 5 minutes prior to particle deposition on the wafer for FE-SEM measurements.

Particle size measurements performed using a Coulter N4 Plus particle analyzer showed mean sizes of $392 \pm 79 \mathrm{~nm}$ and $389 \pm 108 \mathrm{~nm}$ for aminated and plain silica particles respectively. Unimodal analysis was used to determine the mean scattering intensity weighted particle size and standard deviation of the size distribution. The size distribution of particles, shown in figures 3.5 and 3.6, was wider than that obtained using 
FE-SEM measurements. The reasons for this were not clear. However, the mean sizes of particles measured using the two techniques were quite similar.

\subsection{Zeta Potential Measurements}

Zeta potential (ZP) of the particles was measured by a Doppler electrophoretic light scattering technique using a Coulter Delsa 440SX instrument. $\mathrm{KCl}$ was used as a base electrolyte to obtain the desired conductivity of solutions. $\mathrm{HCl}$ and $\mathrm{KOH}$ were used to adjust the $\mathrm{pH}$ of solutions. 

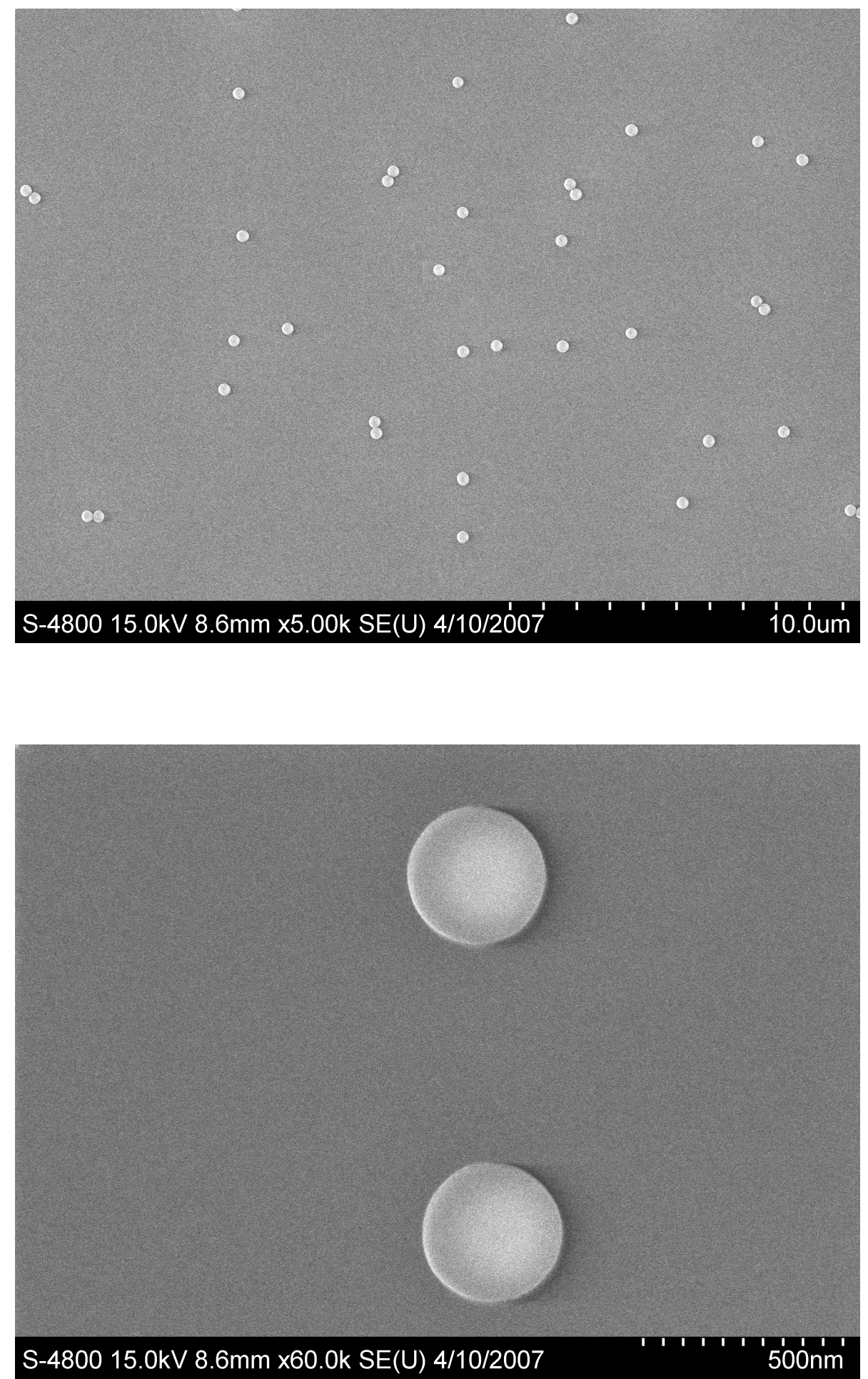

Figure 3.3: FESEM image of aminated silica particles 

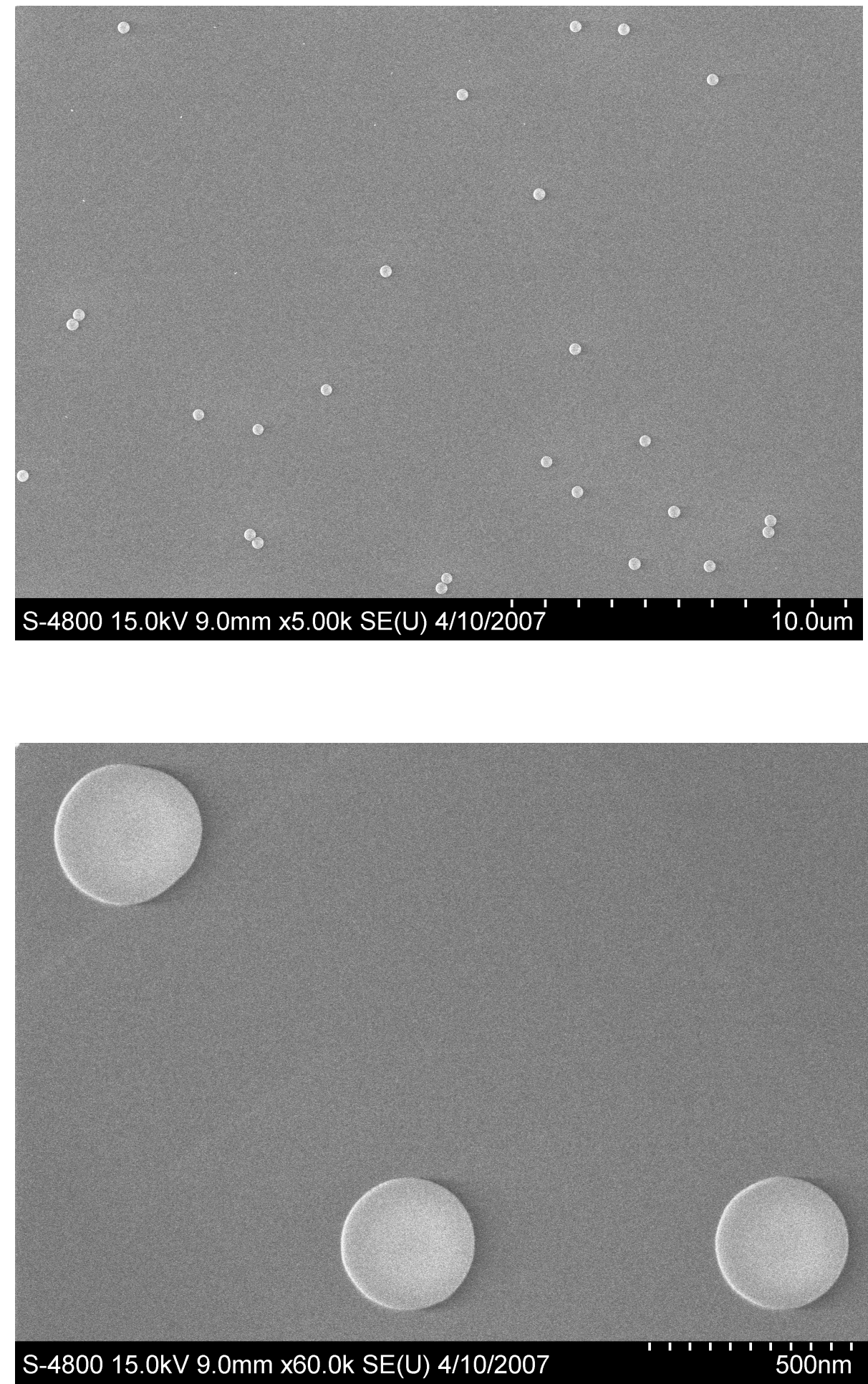

Figure 3.4: FESEM image of plain silica particles 


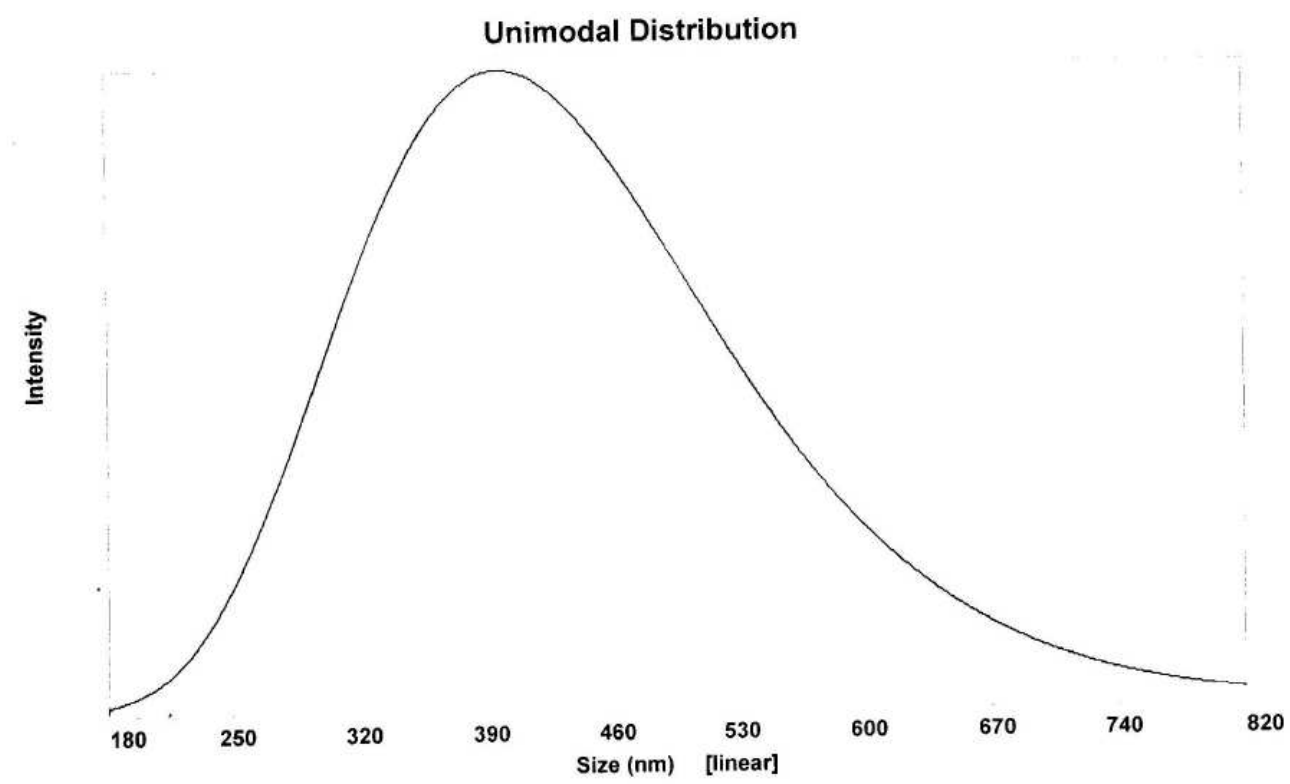

Figure 3.5: Size distribution of aminated silica particles measured using Coulter N4 Plus particle analyzer

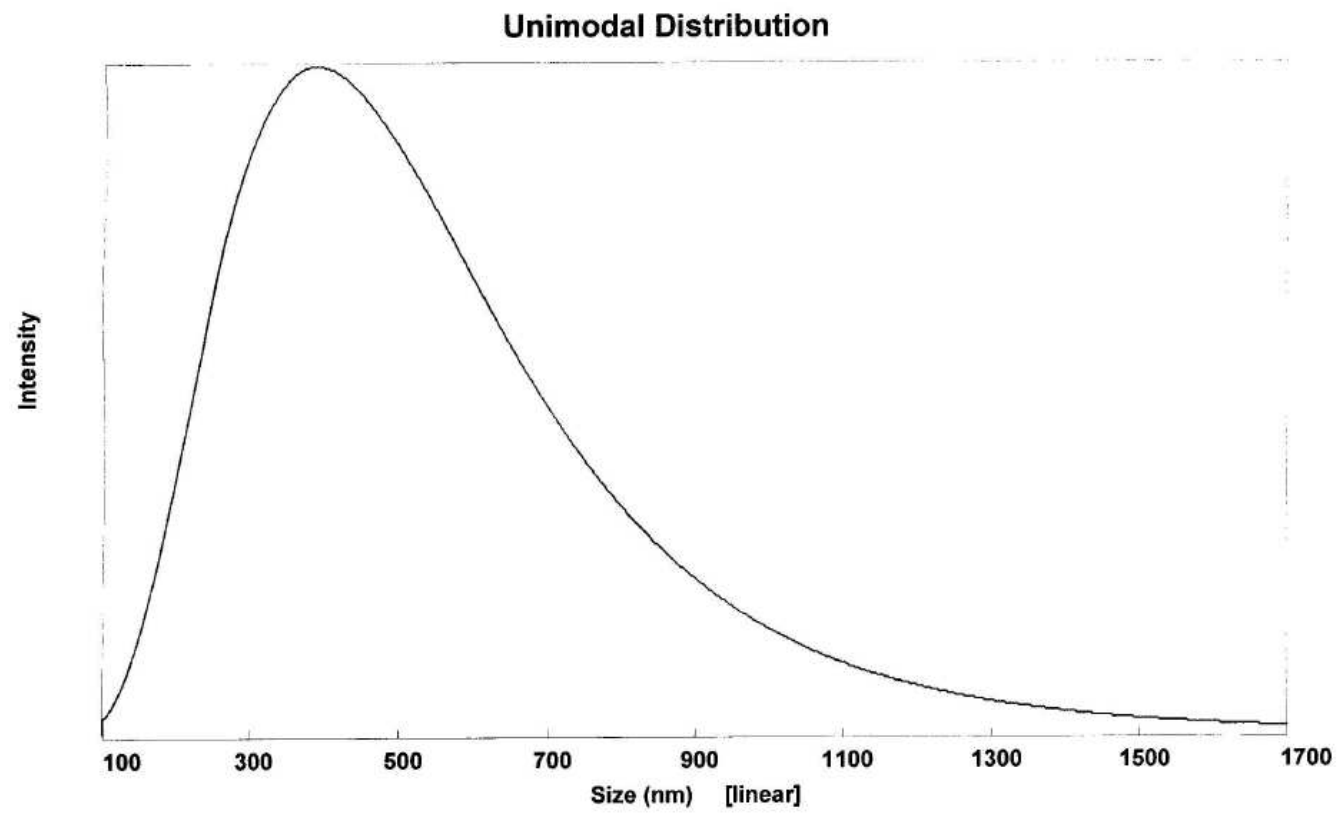

Figure 3.6: Size distribution of plain silica particles measured using Coulter N4 Plus particle analyzer 


\subsection{Wafer Precleaning, Control Deposition and Cleaning}

The wafers were pre-cleaned in a SC1 $\left(1 \mathrm{NH}_{4} \mathrm{OH}: 1 \mathrm{H}_{2} \mathrm{O}_{2}: 50 \mathrm{H}_{2} \mathrm{O}\right)$ bath at $35^{\circ} \mathrm{C}$ with a megasonic power density of $2.17 \mathrm{~W} / \mathrm{cm}^{2}$ for 5 minutes followed by a 30 minutes over flow rinse. A dispersion of contaminating particles was prepared in DI water at a concentration of about $10^{11}$ particles $/ \mathrm{ml}$ and ultra-sonicated for 5 min at $40 \mathrm{kHz}$ using Branson 5510. The wafers were dipped in the dispersion at $25{ }^{\circ} \mathrm{C}$ at a pH of 5.9 and spun at $1000 \mathrm{rpm}$ for $1 \mathrm{~min}$ to deposit approximately 2500 particles. A particle count of less than 200 of unknown origin was present on the wafers prior to contamination. The particles were allowed to age for 24 hours on the wafer prior to cleaning. The wafers were cleaned at $30{ }^{\circ} \mathrm{C}$ for 1 minute in all the cleaning experiments, unless mentioned otherwise. The wafers were inspected by a light scattering technique using SURFSCAN 5500. This technique uses scattered laser light (helium-neon laser, $5 \mathrm{~mW}$, wavelength $=$ $632.8 \mathrm{~nm}$ ) to analyze reflections and allocates the particles in different bin sizes based on the scattering cross-sectional area from $0.003 \mu \mathrm{m}^{2}$ to $1024 \mu \mathrm{m}^{2}$. The scattering cross section is the ratio of the power scattered by the particle to the power density incident upon it. Since the intensity of light scattered from a particle depends on the size of the particle, its shape, the reflectivity of the substrate, and the optical properties of the particle (particularly the dielectric constant), scattered light intensity cannot be uniquely related to particle size. Figures 3.7 and 3.8 show representative wafer maps after precleaning and deposition steps. Typical histograms showing particle size distribution in different bins after pre-cleaning and deposition steps are also shown in figures 3.9 and 
3.10 respectively. Particle counts for the bins $0.066-0.112 \mu \mathrm{m}^{2}$ were considered for calculating the particle removal efficiency (PRE), as the bin corresponds to the size of the deposited particles. The PRE was calculated as follows,

$$
\operatorname{PRE}(\%)=\frac{(\# \text { of particles after deposition }-\# \text { of particles after cleaning })}{(\# \text { of particles after deposition })} \times 100
$$


Figure 3.7: Image of 150-mm Si wafer after pre-cleaning step using SURFSCAN 5500

Figure 3.8: Image of 150-mm Si wafer deposited with aminated silica particles $(367 \pm 20$ nm) using SURFSCAN 5500 


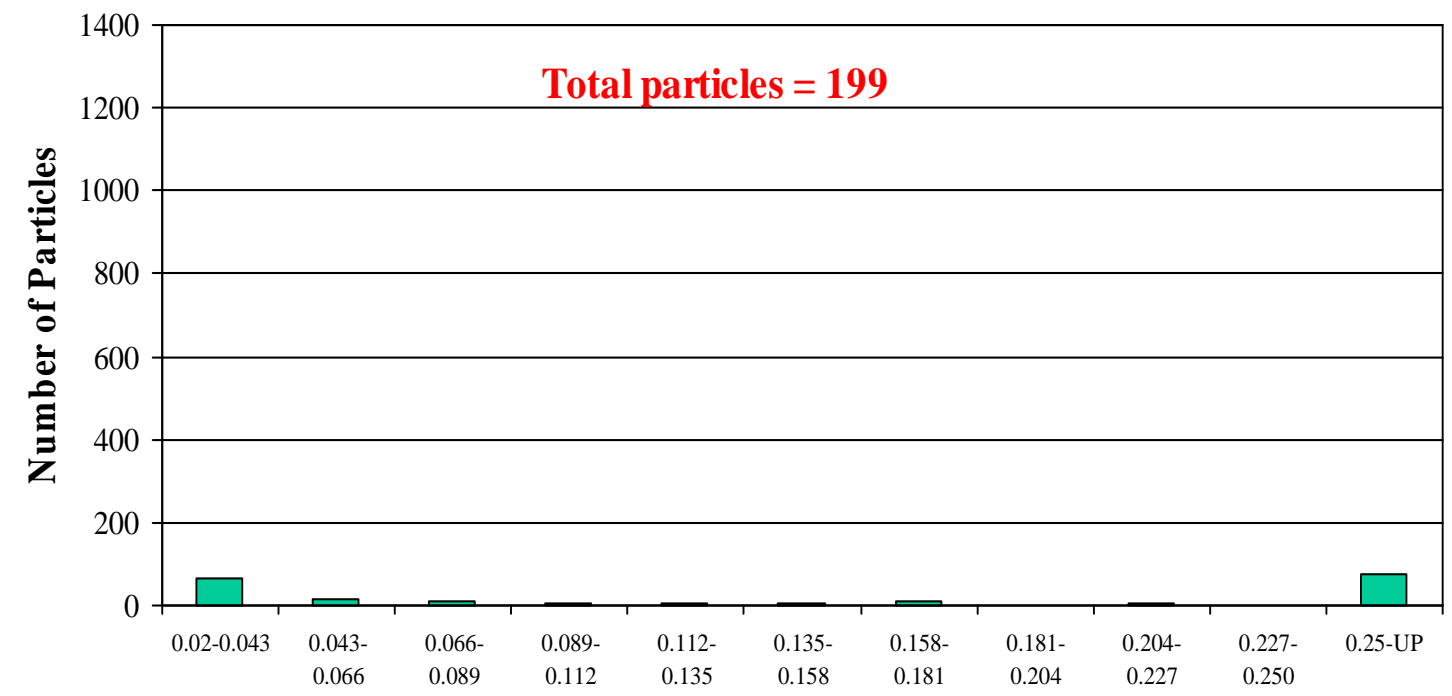

Particle Cross Section Area (micrometer ${ }^{2}$ )

Figure 3.9: Histogram showing distribution of particles in different bin sizes after precleaning step

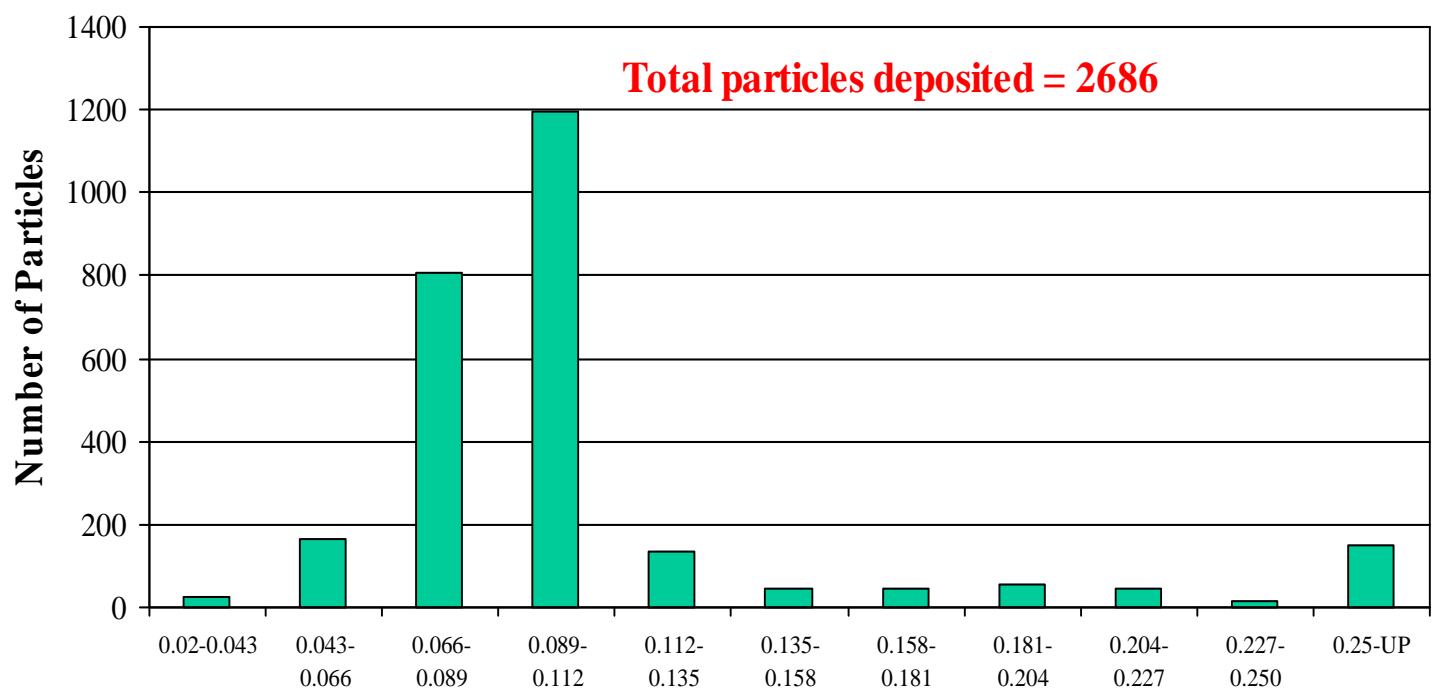

Particle Cross Section Area (micrometer ${ }^{2}$ )

Figure 3.10: Histogram showing distribution of aminated silica particles $(367 \pm 20 \mathrm{~nm})$ in different bin sizes after deposition step 


\subsection{Cavitation Measurements}

Real time monitoring of cavitation density was performed in the megasonic tank using a cavitation probe (MicroProbe System ${ }^{\mathrm{TM}}$ ) donated by ProSys Inc. A custom designed positioning system was used to control the location of the probe in the tank. A schematic of the probe is shown in figure 3.11 [3.4].

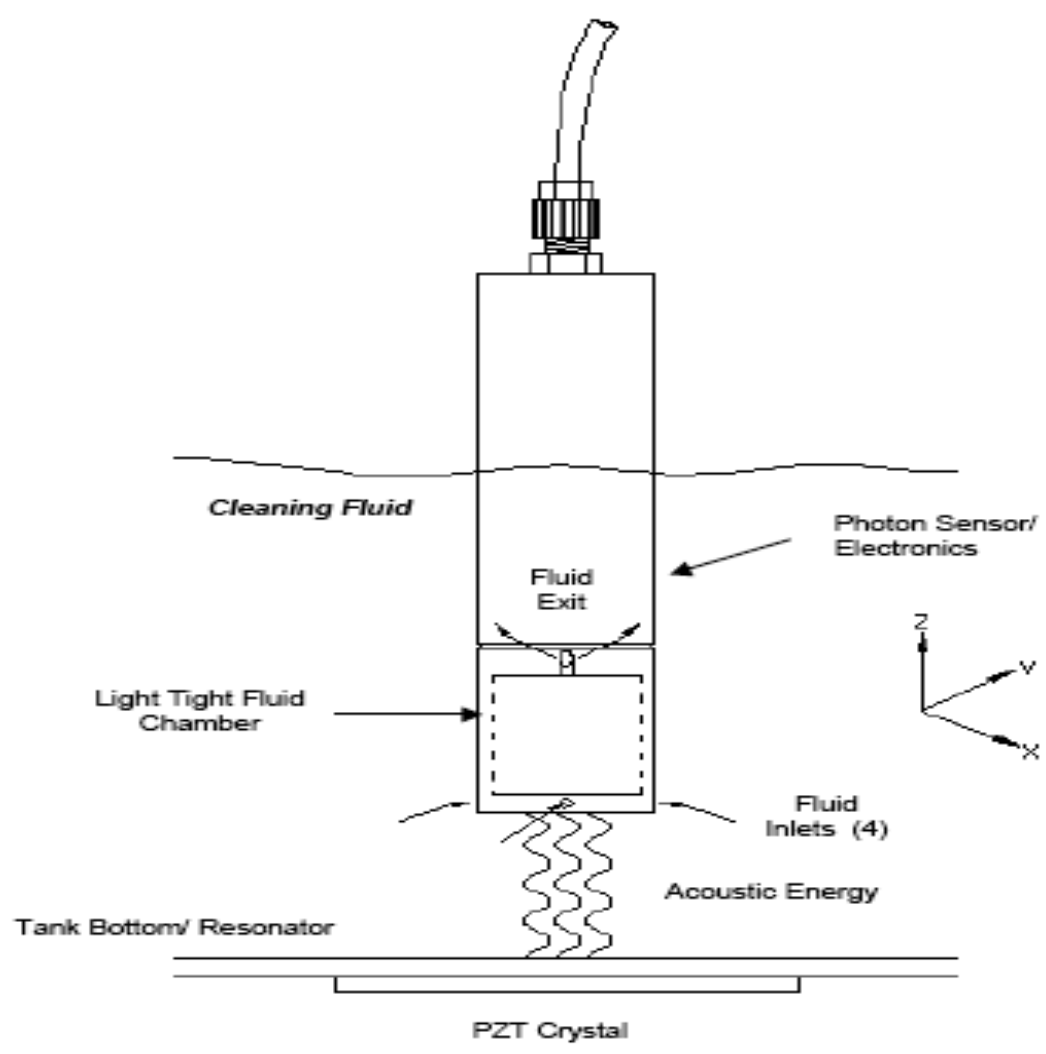

Figure 3.11: Schematic of the cavitation probe [3.4] 
The probe consists of a unique chloro-polyvinyl chloride (CPVC) sensor head that allows the local fluid to enter the system through a tortuous path in the light proof chamber. A simple path, even with a low reflection coefficient, can allow the visible light photons to enter the cell and increase the background photon counts at a significant level. A thin tantalum membrane $(100 \mu \mathrm{m})$, located at the bottom of the probe, is transparent to the acoustic field but completely opaque to external photons. It is also thick enough to prevent any damage that might be caused due to cavity implosion during cavitation measurements. The probe design produces a low background count of 100 photons/s or less.

The probe measures the cavitation in the megasonic system by detecting the light emitted by sonoluminescence. When the fluid in the chamber is exposed to acoustic energy, the emission of photons occurs due to the cavitation in fluid. The photons are captured by the photo multiplier tube (PMT) that is sensitive to a photon wavelength in the range of 270 to $650 \mathrm{~nm}$. The photons are detected by the sensor and counted by electrical circuitry. The counts are communicated to the computer via an RS232 connection.

The photon count changes with the position as well as with the angle of inclination of the probe in the tank. During measurements, the probe was positioned vertically in the far field region of the transducers and at the same location in the tank within a precise measurement of $0.03 \mathrm{~mm}$. The thickness of the near field region in DI water as calculated 
for the transducer design using the equation $\mathrm{a}^{2} / 4 \lambda$ ( $\mathrm{a}$ is the width of the transducer, 1 inch, and $\lambda$ is the wavelength of the sound wave in DI water, $1.48 \mathrm{~mm}$ ) is $10.9 \mathrm{~cm}$.

\subsection{Hydrophone Measurements}

Hydrophone measurements were performed using a 2-mm needle shaped hydrophone (PVDF-Z44-1500 Onda Corp.) that tapered at the bottom to a pressure sensitive area with a diameter of about $1.5 \mathrm{~mm}$. The total length of the hydrophone was approximately 2.5 inches. The hydrophone was located vertically about 8 inches from the surface of the transducer. The horizontal position of the hydrophone in the tank was not precisely controlled for these experiments; hence a reference measurement in DI water was always carried out at the same location where a measurement for experimental solution was performed for comparison purposes. The hydrophone was connected to a NI PCI-5102 scope, with the set-up designed to acquire 65000 continuous samples for each run using LabVIEW 6.1. The sampling rate was 20 million samples/s. The output signal, in volts, from the oscilloscope was converted to the root mean square (RMS) value ( $\mathrm{V}_{\mathrm{RMS}}$ ) for a comparison of results between different solutions. These RMS voltages were proportional to the pressure amplitude of the sound wave. 


\section{CHAPTER 4: RESULTS AND DISCUSSION}

As a first step in this work, the zeta potential (ZP) of aminated and plain silica particles was determined to understand the nature (positive or negative) of surface charge on these particles at different solution $\mathrm{pH}$ values.

\subsection{Zeta Potential Measurements of Plain and Aminated Silica Particles}

The $\mathrm{ZP}$ of silica particles at different solution $\mathrm{pH}$ values is displayed in figure 4.1. At the deposition $\mathrm{pH}$ of 5.9, the $\mathrm{ZP}$ of plain silica and aminated silica particles was about -20 and $+40 \mathrm{mV}$ respectively, based on the interpolation of data measured at other $\mathrm{pH}$ values. The isoelectric point (IEP) of plain silica and aminated silica particles was found to occur at $\mathrm{pH}$ values of 3.9 and 7.1 respectively. 


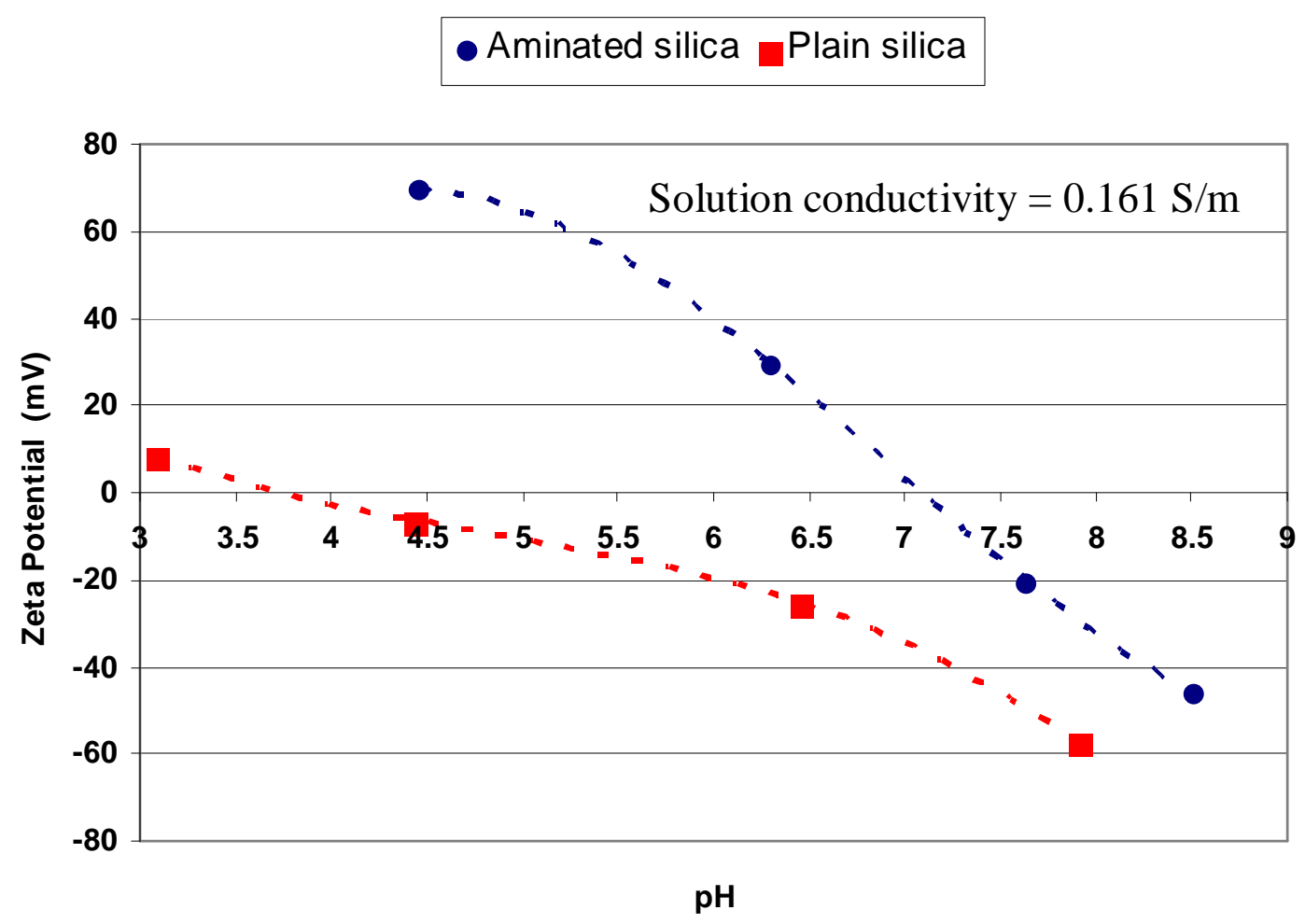

Figure 4.1: ZP of plain and aminated silica particles 


\subsection{Megasonic Cleaning in Potassium Chloride Solutions}

In the first series of cleaning experiments, plain silica particles, characterized by a negative $\mathrm{ZP}$ at the deposition $\mathrm{pH}$ of 5.9, were used as contaminants. Wafers were cleaned in DI water and a potassium chloride $(\mathrm{KCl})$ solution of different concentrations at a megasonic power density of $0.077 \mathrm{~W} / \mathrm{cm}^{2}$. The results, shown in figure 4.2 , indicate that in DI water alone, only $20 \%$ of the particles are removed. The addition of $\mathrm{KCl}$ does not improve cleaning efficiency until a critical $\mathrm{KCl}$ concentration is reached. By defining this critical concentration $\left(\mathrm{C}_{\mathrm{c}}\right)$ as the concentration at which $50 \%$ particle removal efficiency (PRE) is achieved, for silica particles with negative $\mathrm{ZP}, \mathrm{C}_{\mathrm{c}}$ is about $0.05 \mathrm{M}$ at a power density of $0.077 \mathrm{~W} / \mathrm{cm}^{2}$. A PRE of about $85 \%$ can be achieved in $0.5 \mathrm{M} \mathrm{KCl}$. In the absence of a megasonic field, no removal of particles from the wafer surface was observed even in $1 \mathrm{M} \mathrm{KCl}$ solution. 


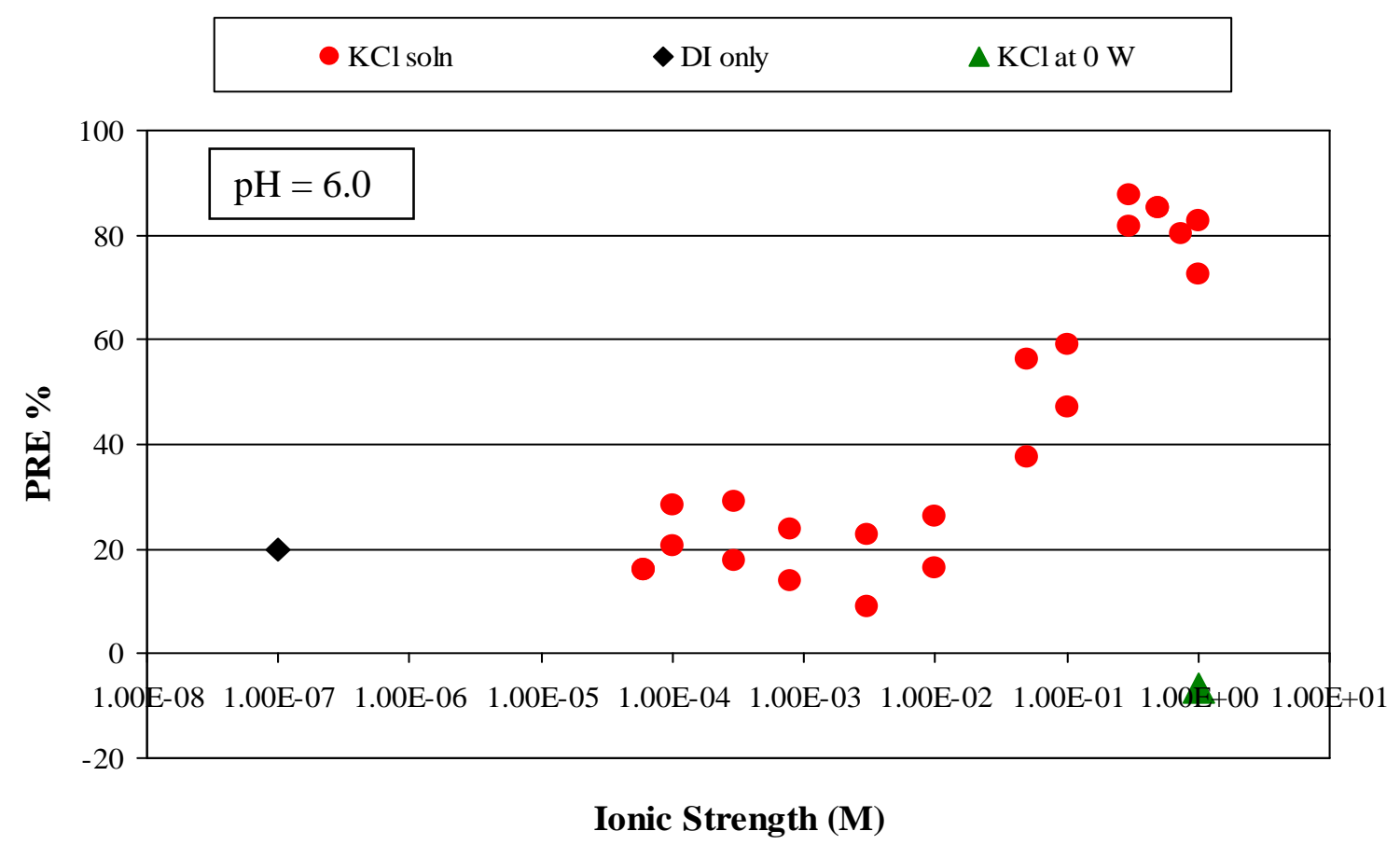

Figure 4.2: Removal of plain silica particles from wafer surface in $\mathrm{KCl}$ solution of different concentrations at $30{ }^{0} \mathrm{C}$ and power density of $0.077 \mathrm{~W} / \mathrm{cm}^{2}$

The effect of power density on cleaning performance was investigated in both 0.57 $\mathrm{M} \mathrm{KCl}$ solution and DI water. The results are shown in figure 4.3. As may be clearly seen from this figure, the removal of particles in $\mathrm{KCl}$ solution is significantly larger than in DI water at all power densities. The dependence of PRE on power density in $\mathrm{KCl}$ solution is not very significant beyond $0.073 \mathrm{~W} / \mathrm{cm}^{2}$. PREs greater than $90 \%$ can be achieved in $\mathrm{KCl}$ solutions. 


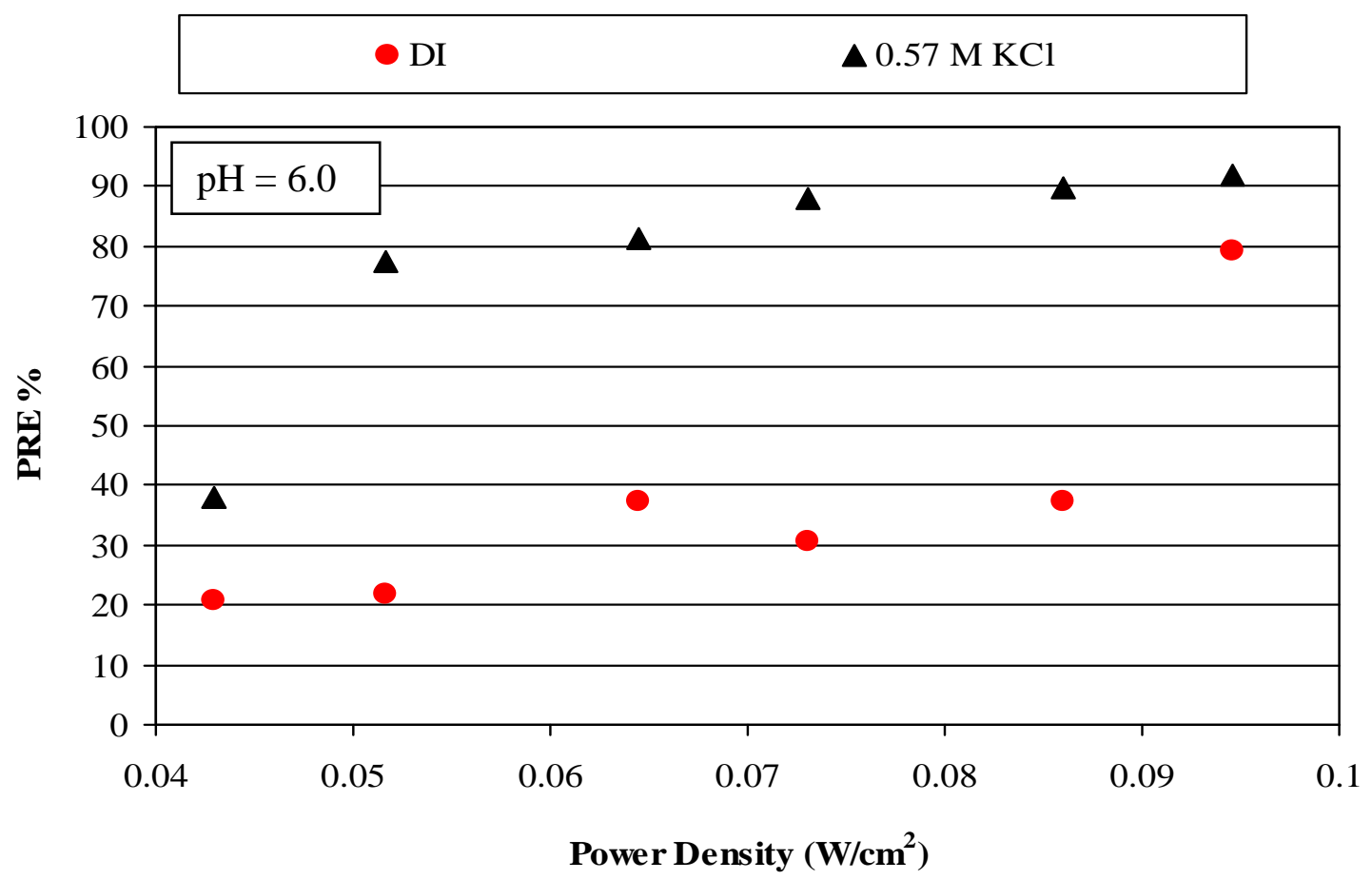

Figure 4.3: Removal of plain silica particles from wafer surface in $0.57 \mathrm{M} \mathrm{KCl}$ solution and DI water at $30{ }^{\circ} \mathrm{C}$

Negatively charged silica particles are not expected to adhere very strongly to the silica surface, which is also negatively charged at a deposition $\mathrm{pH}$ of 5.9. This is evident from figure 4.3, where at power densities larger than $0.09 \mathrm{~W} / \mathrm{cm}^{2}$, the PRE is close to $90 \%$. In order to investigate the effect on particles that may be strongly adhered to the wafer surface, a series of tests was performed using positively charged aminated silica particles (characterized by a positive $\mathrm{ZP}$ at the deposition $\mathrm{pH}$ ). As shown in figure 4.4, DI water alone exhibits a PRE of only $6 \%$ at $0.43 \mathrm{~W} / \mathrm{cm}^{2}$. The addition of $\mathrm{KCl}$ increases the PRE. 


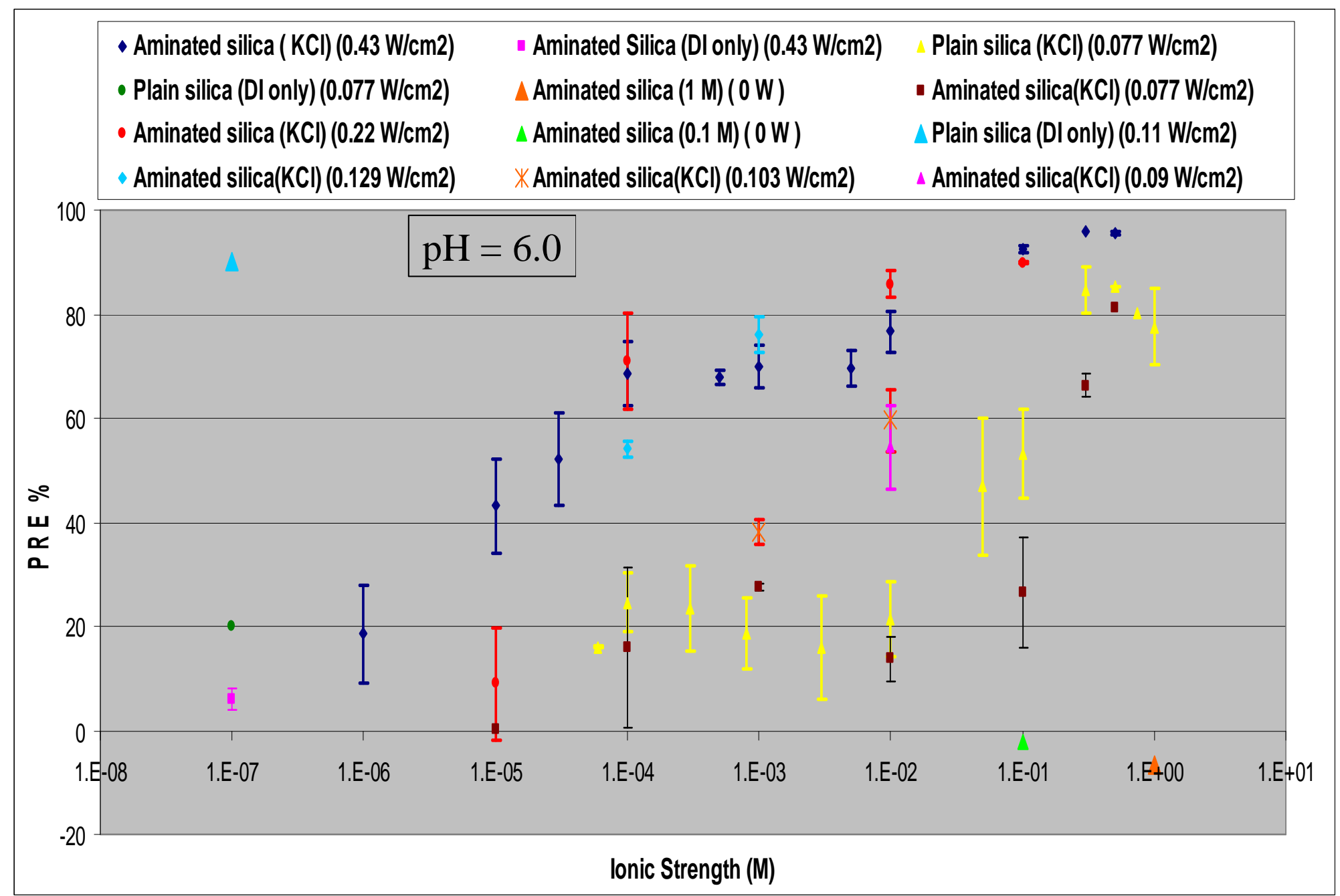

Figure 4.4: Removal of aminated and plain silica particles from wafers in $\mathrm{KCl}$ solutions of different concentration 
A critical concentration of $3 \times 10^{-5} \mathrm{M}$ was observed at a power density of 0.43 $\mathrm{W} / \mathrm{cm}^{2}$. Similarly, at a lower power density of $0.077 \mathrm{~W} / \mathrm{cm}^{2}, \mathrm{C}_{\mathrm{c}}$ was found to be $0.2 \mathrm{M}$, much higher than that at $0.43 \mathrm{~W} / \mathrm{cm}^{2}$. Data in figure 4.4 clearly show that higher PREs are feasible at lower power densities by increasing the $\mathrm{KCl}$ concentration. The use of lower power densities is likely to be beneficial in reducing the damage to features in the cleaning of patterned wafers.

Critical concentrations of $\mathrm{KCl}$ at different power densities, shown in figure 4.5, indicate that $\mathrm{C}_{\mathrm{c}}$ increases with decreasing power density. The slope of the curve is steeper at lower power densities suggesting that particle removal is more sensitive to $\mathrm{KCl}$ concentration at higher power density than at lower power densities.

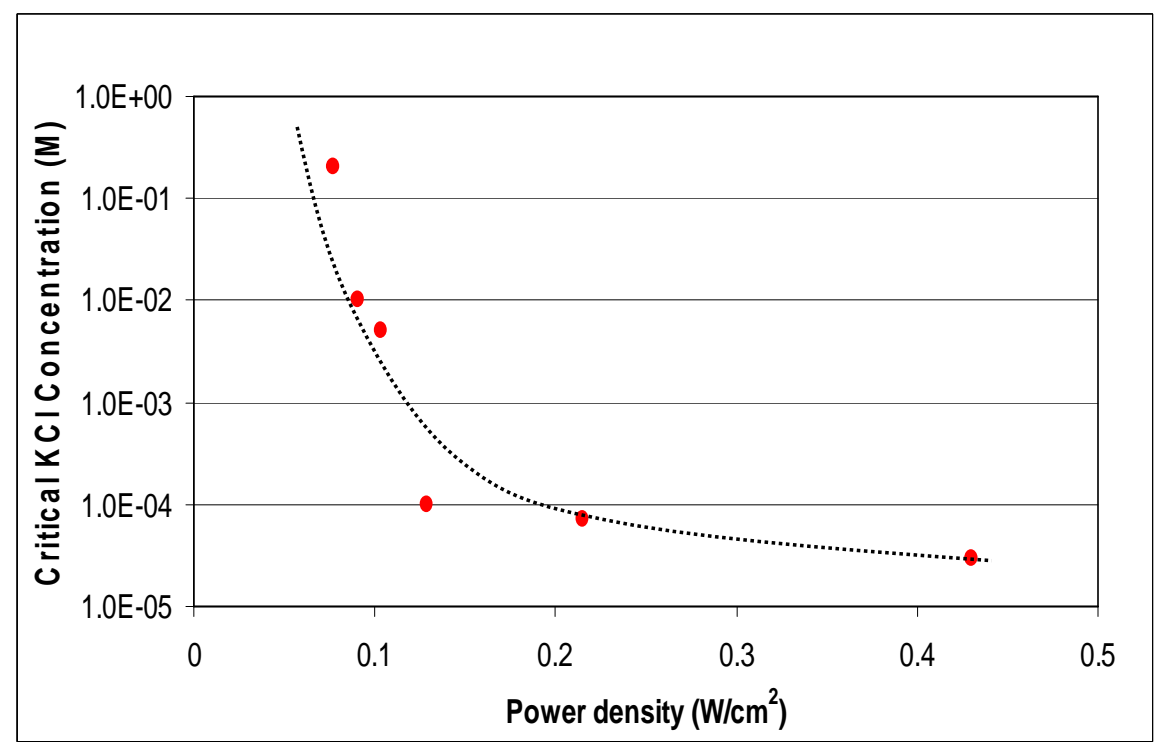

Figure 4.5: Critical concentration of $\mathrm{KCl}$ at $30{ }^{\circ} \mathrm{C}$ for removal of aminated silica particles from wafer surface at different power densities 


\subsubsection{Possible Role of Electro-Acoustic Effects on Removal of Silica Particles from Wafer Surfaces}

In order to understand the experimentally observed effect of enhanced removal of charged particles from wafer surfaces in ionic solutions irradiated with megasonic energy, forces on particles due to the electro-acoustic effects were calculated. As a first step in this direction, a theoretical computation of the effect of solution ionic strength and transducer intensity on the magnitude of Ionic Vibration Potential was performed. The maximum potential generated, $\phi_{0}$, in $\mathrm{KCl}$ solutions of different concentrations at $25{ }^{0} \mathrm{C}$ and power densities ranging from $0.077 \mathrm{~W} / \mathrm{cm}^{2}$ to $2.17 \mathrm{~W} / \mathrm{cm}^{2}$ was calculated using equation 2.27. The frictional coefficients and the hydrated masses of the ions were obtained from [2.27] and [2.29] respectively. As displayed in figure 4.6, the potential increases with power density and $\mathrm{KCl}$ concentration, but saturates at $10^{-3} \mathrm{M} \mathrm{KCl}$ concentration. These values are consistent with experimentally measured values of IVP or Debye potential [2.29].

The force experienced by a charged particle with a specific charge density due to IVP is the product of charge density multiplied by the electric field due to IVP. Considering that IVP acts along a distance of $\lambda / 2 \pi$, the intensity of force is $1.7 \times 10^{-15} \mathrm{~N}$ for a particle with a charge density of $2 \mu \mathrm{C} / \mathrm{cm}^{2}$ (on aminated silica particles at $\mathrm{pH}$ of 6.0 ) in $10^{-3} \mathrm{M} \mathrm{KCl}$ solution at a power density of $2.17 \mathrm{~W} / \mathrm{cm}^{2}$. The van der Waals force of adhesion between the aminated silica particle and the wafer surface was computed using 
equation 2.3. The values of $A_{132}$ required for the calculation of van der Waals force was evaluated using Hamaker constants for silica $\left(A_{11}\right.$ and $\left.A_{33}\right)$ and water $\left(A_{22}\right)$ as $6.2 \times 10^{-20}$ and $4.4 \times 10^{-20} \mathrm{~J}$ respectively [Table 2.1]. Assuming $4 \AA$ of separation distance [1.43, 2.14] between the silica particle and the wafer surface, the van der Waals force is found to be $3 \times 10^{-10} \mathrm{~N}$. A comparison of removal and adhesion forces shows that the Debye effect does not produce an adequate force to cause removal of particles from the surface.

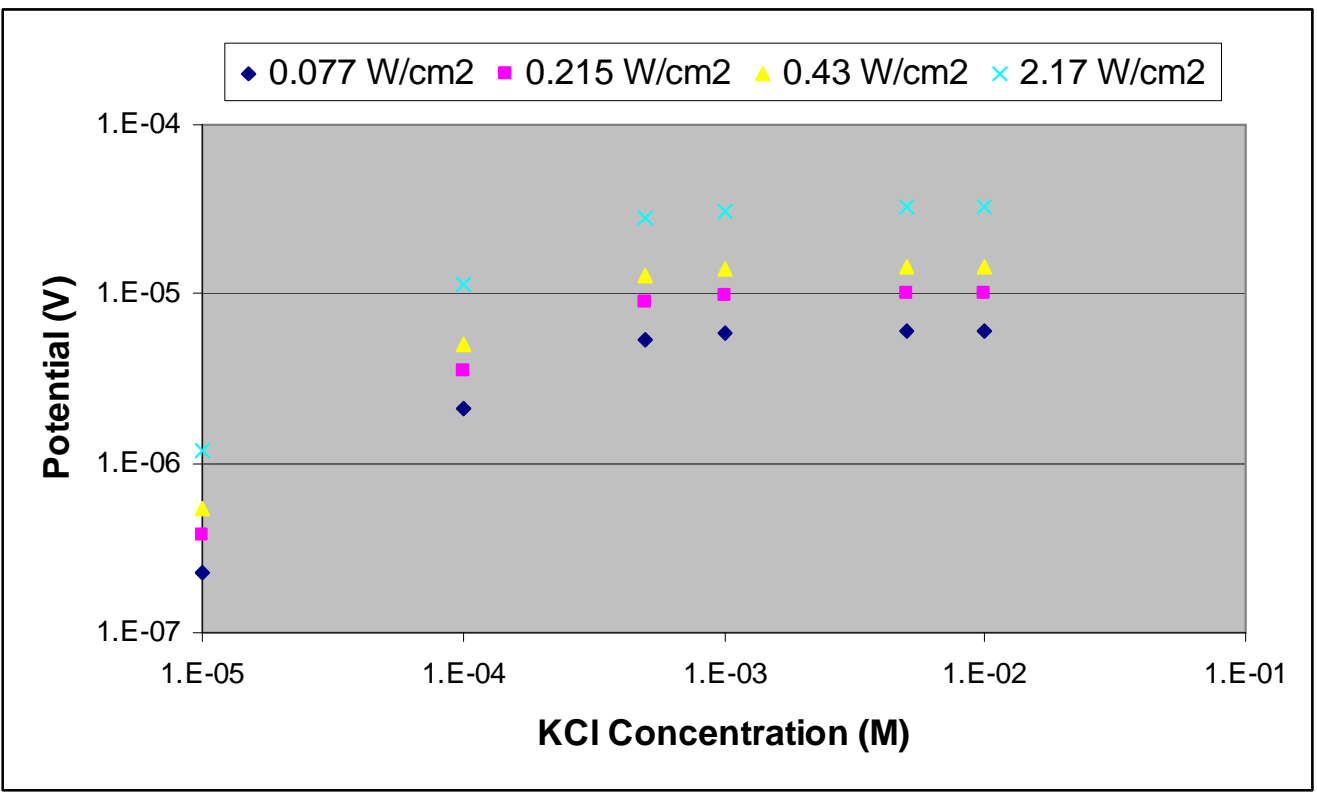

Figure 4.6: Debye potential in $\mathrm{KCl}$ solutions of different concentration and power densities

The next step was to compute the magnitude of force due to the formation of a dipole moment around the charged particle in an acoustic field. Initially, the force calculations were performed at a transducer intensity of $0.43 \mathrm{~W} / \mathrm{cm}^{2}$, where the value of $\mathrm{C}_{\mathrm{c}}$ is $3 \times 10^{-5} \mathrm{M}$ for aminated silica particles. In the case of aminated silica particles adhered to the wafer surface, the back flow of ions can be ignored due to the following 
reason. Consider a stationary aminated silica particle suspended in electrolyte solution subjected to an acoustic field. Due to relative displacement of the particle and the double layer, the net charge density at the rear end of the particle increases as shown in figure 4.7. This causes a back flow of ions in the continuous phase to neutralize this surface charge.

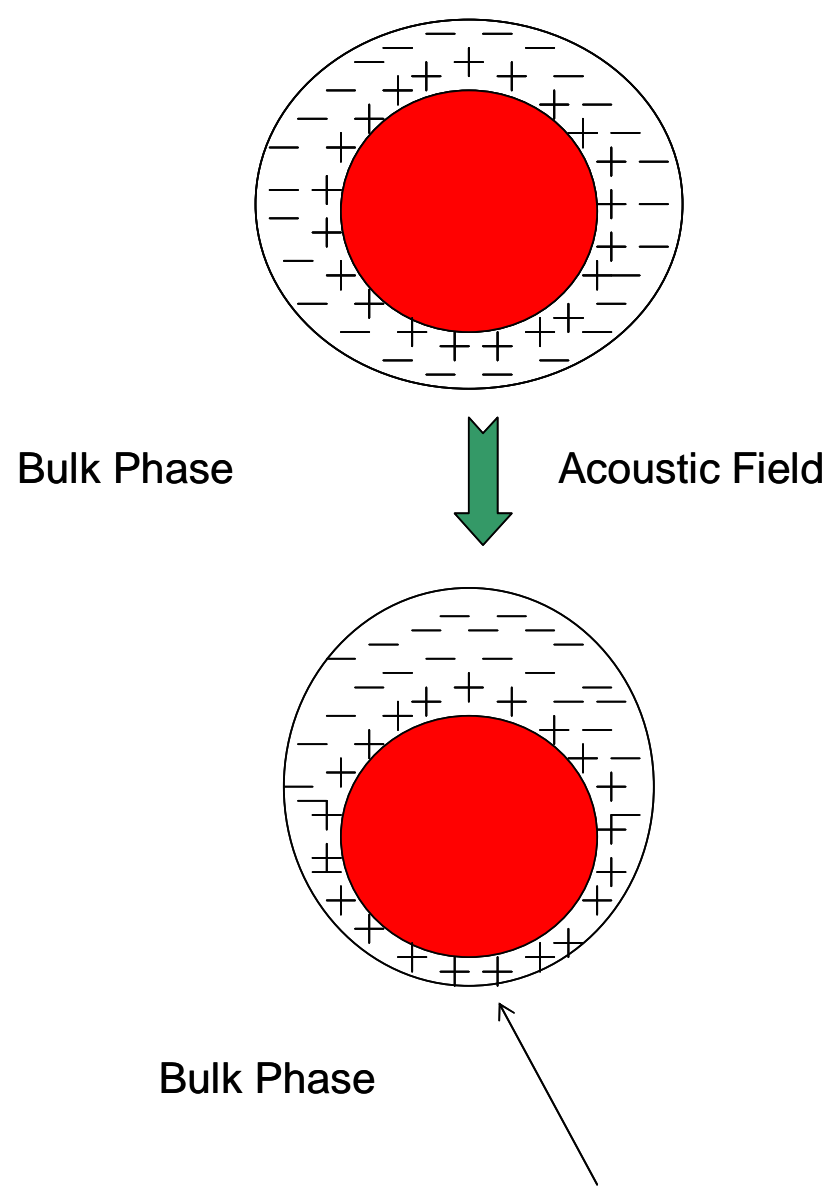

Back Flow of lons

Figure 4.7: Schematic of back flow of ions in the case of aminated silica particle suspended in electrolyte solution subjected to acoustic field 
When the same particle is adhered on an oppositely charged silica surface, the transport of ions is constrained by the wafer surface and the associated double layer as illustrated in figure 4.8. Therefore, equation 2.39 may be used safely for all dipole moment calculations.
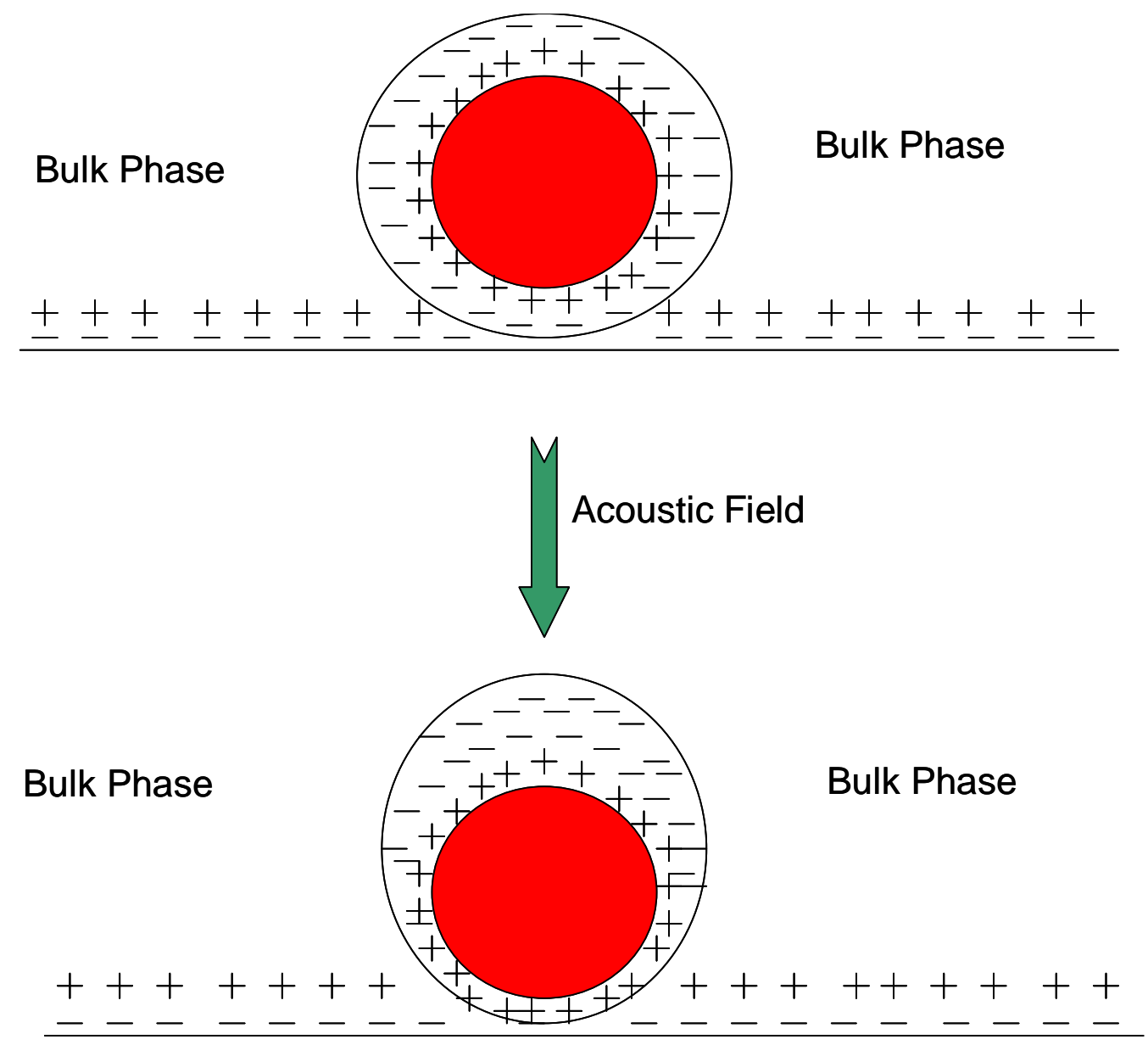

Figure 4.8: Schematic showing reduced back flow of ions for aminated silica particle adhered to a wafer surface immersed in an electrolyte solution subjected to acoustic field 
Since the particles are stationary (adhered to the wafer surface), $v$ in equation 2.38 can be taken approximately as the maximum velocity of the fluid, $U$, computed using equation 2.39. For a solution of given ionic strength maintained at a particular $\mathrm{pH}$, the value of the dipole moment depends on the zeta potential. The zeta potential of aminated silica particles was measured as a function of $\mathrm{pH}$ at only one value of ionic strength. However, cleaning experiments were done at $\mathrm{pH} 6$ over many decades of ionic strengths. Measurement of $\mathrm{ZP}$ values at very low ionic strength was difficult as $\mathrm{pH}$ could not be changed without changing the ionic strength. Measurements at very high ionic strengths created thermal overturn effects. Hence, an attempt was made to estimate ZP values from surface charge density values.

4.2.1.1. Estimation of Zeta Potential from the Surface Charge Density of Aminated Silica Particles

The protocol used to estimate ZP was to first compute the surface potential of aminated silica particles at $\mathrm{pH}$ of 4.5 , using equation $2.17 \mathrm{~b}$, from the calculated value of surface charge density. The computed value of surface potential and measured $\mathrm{ZP}$ at $\mathrm{pH}$ of 4.5 were used to obtain the distance of the shear plane using equation 2.17 a. The computed value of the shear plane distance can be used to calculate ZP at any value of $\mathrm{pH}$ and ionic strength following a similar procedure. 
The surface charge density of aminated silica particles was estimated as follows. The aminated silica particles were prepared by surface modification of silica particles achieved through grafting of amino silane on the silica surface. In this process, three adjacent silanol groups (site density of 2-3 $\mathrm{SiOH}$ groups per $\mathrm{nm}^{2}$ ) of original silica particles were modified into one amino group. Depending on the extent of surface coverage of amino groups, the charge density of these particles can vary from 5 to 15 $\mu \mathrm{C} / \mathrm{cm}^{2}$ at $\mathrm{pH}$ of 4.5 , assuming complete protonation of amine group at this $\mathrm{pH}$. It must also be noted that these particles, due to the amino groups on the surface, can be considered to possess a constant surface charge at a specified $\mathrm{pH}$. However, the potential of such a surface is a function of ionic strength of the solution.

Considering a surface charge density of $5 \mu \mathrm{C} / \mathrm{cm}^{2}$, at a solution $\mathrm{pH}$ of 4.5 , the surface potential can be calculated as a function of solution ionic strength using equation 2.17 b. The surface potential decreases as a natural logarithmic of ionic strength, as shown in figure 4.9 . 


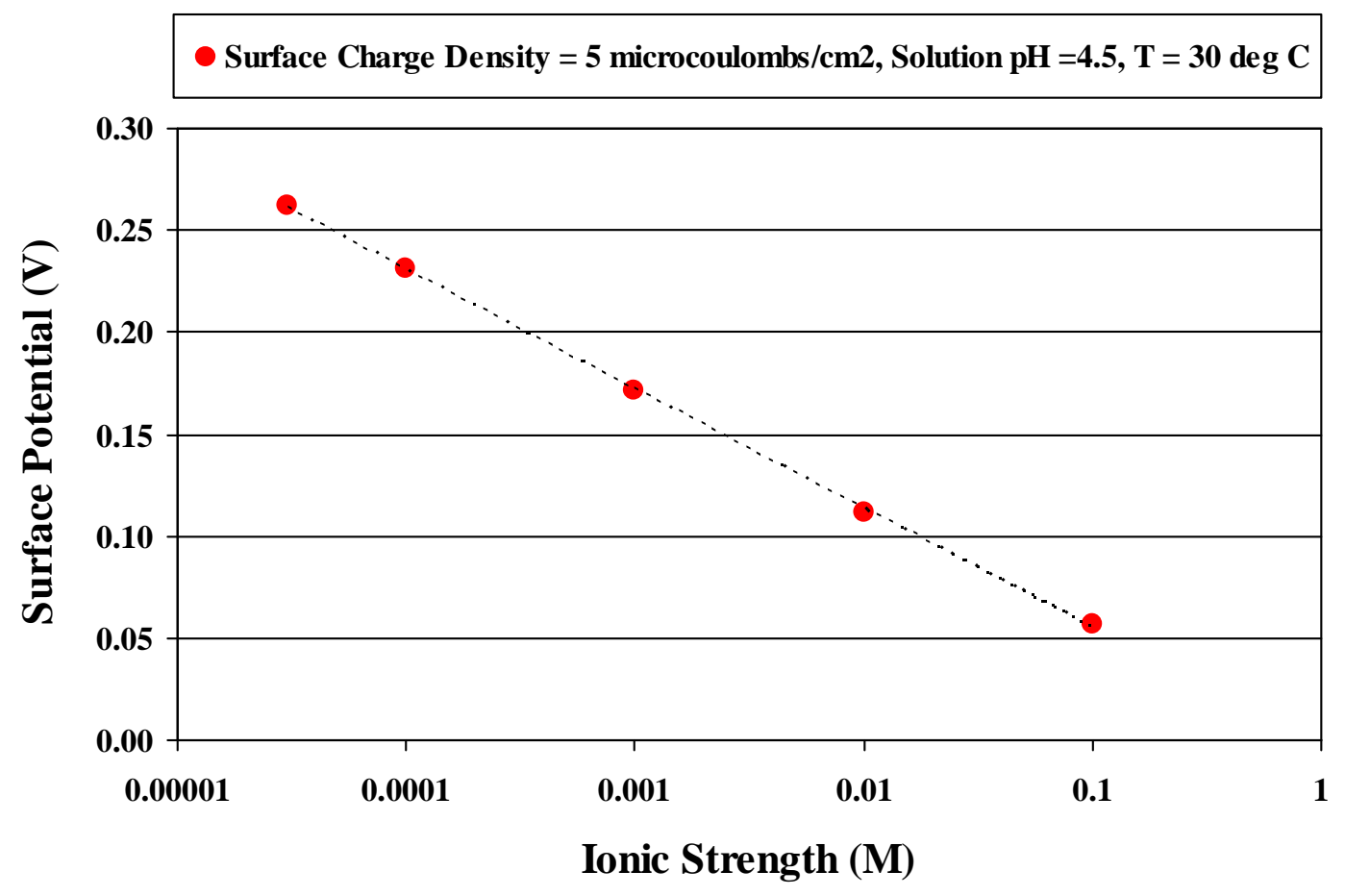

Figure 4.9: Surface potential of aminated silica particles at solution $\mathrm{pH}$ of 4.5 and temperature of $30{ }^{\circ} \mathrm{C}$

Since surface potential and zeta potential are now known at a $\mathrm{pH}$ of 4.5 and ionic strength of $0.01 \mathrm{M}$ from figures 4.9 and 4.1 respectively, the distance of the shear plane from the surface can be computed as $9 \AA$, using equation $2.17 \mathrm{a}$.

In order to determine the zeta potential values as a function of ionic strength at a solution $\mathrm{pH}$ of 6.0 , the surface potentials were first determined for these conditions. The charge density at this $\mathrm{pH}$ was computed to be $\sim 2 \mu \mathrm{C} / \mathrm{cm}^{2}$ from interpolation of surface charge densities of zero and $5 \mu \mathrm{C} / \mathrm{cm}^{2}$ at $\mathrm{pH}$ values of 7.1 and 4.5 , respectively. By substituting these surface potential values in equation $2.17 \mathrm{a}$, zeta potentials were obtained 
as a function of ionic strength for a shear plane distance of $9 \AA$. These values are plotted in figure 4.11. The value of zeta potential calculated as $47 \mathrm{mV}$ at $0.01 \mathrm{M}$, compared well with the experimentally determined value of $43 \mathrm{mV}$. Also the dependency of zeta potential on ionic strength was a natural logarithmic with higher ionic strengths resulting in lower zeta potentials.

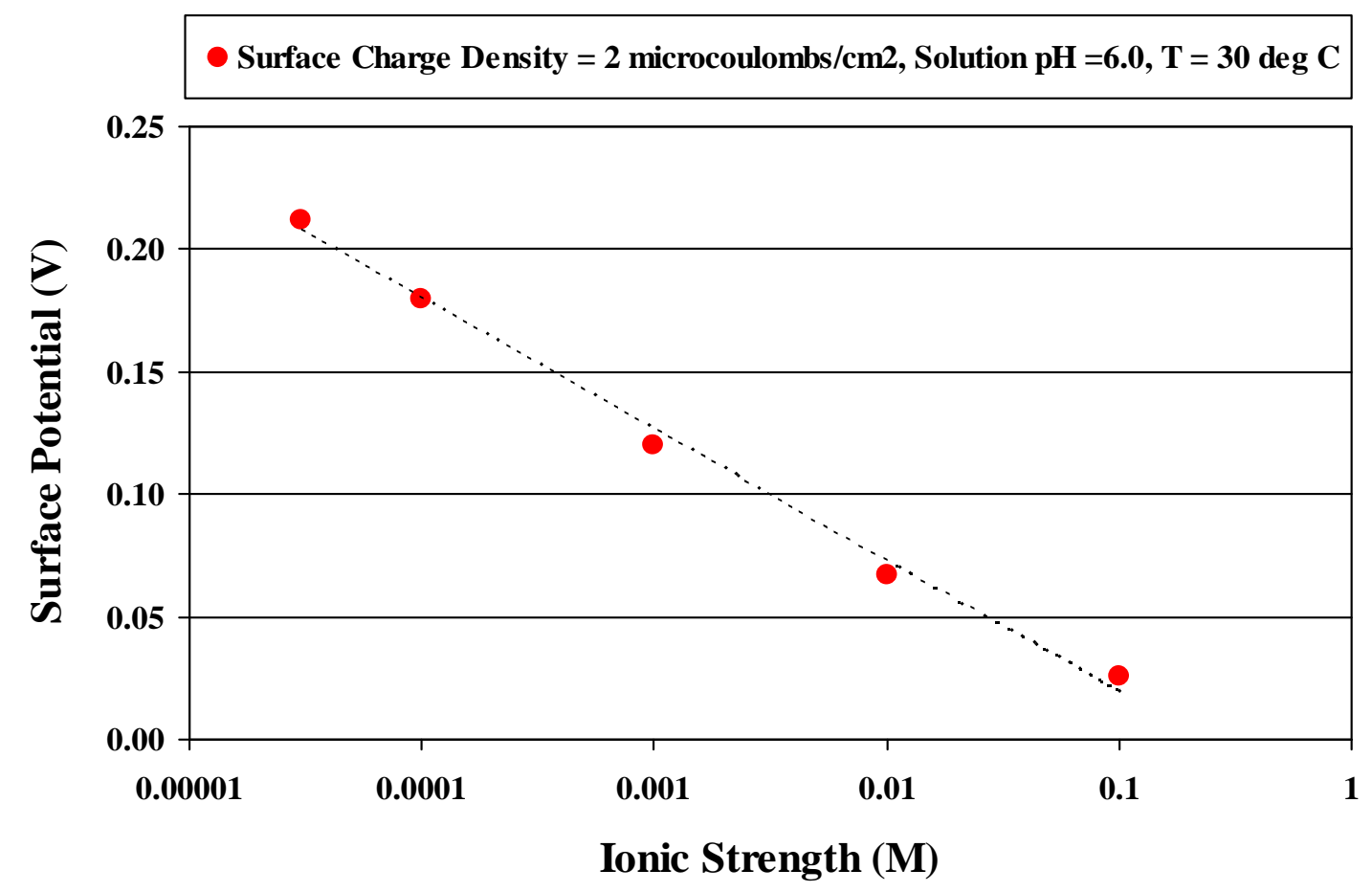

Figure 4.10: Surface potential of aminated silica particles at solution $\mathrm{pH}$ of 6.0 and temperature of $30{ }^{0} \mathrm{C}$ 


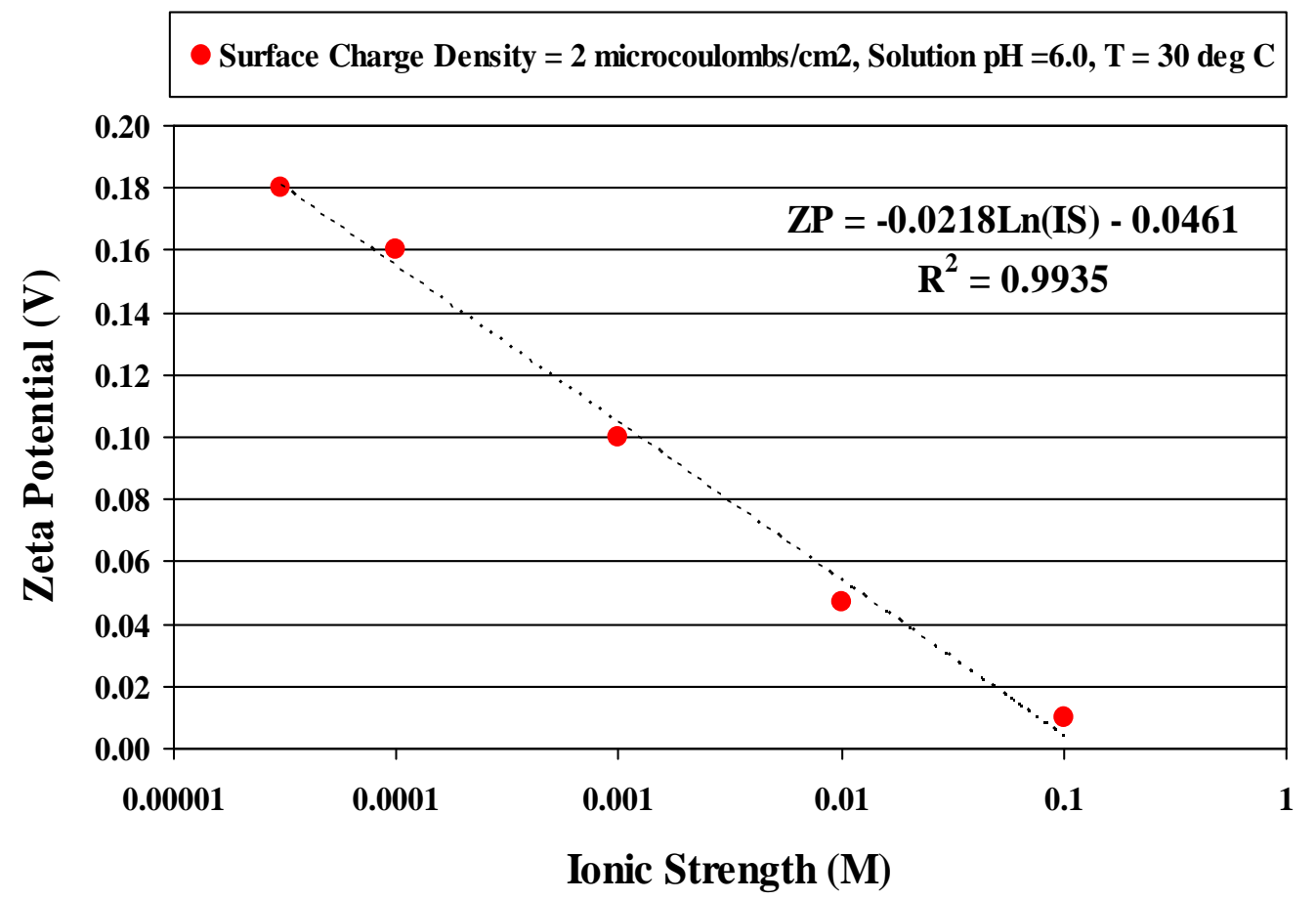

Figure 4.11: Zeta potential of aminated silica particles at solution $\mathrm{pH}$ of 6.0 and temperature of $30^{\circ} \mathrm{C}$

The dipole moment, $m$, computed using equation 2.40 , for transducer power density of $0.43 \mathrm{~W} / \mathrm{cm}^{2}$ and ionic strength of $3 \times 10^{-5} \mathrm{M}$, was found to be $5.8 \times 10^{-24} \mathrm{C}-\mathrm{m}$. The calculation of force, $F$, requires $Q^{\prime}$ and $l$ which are not known. Different values of Q' were obtained by taking the ratio of $m$ to $l$ for different values of $l$, as shown in figure 4.12 . 


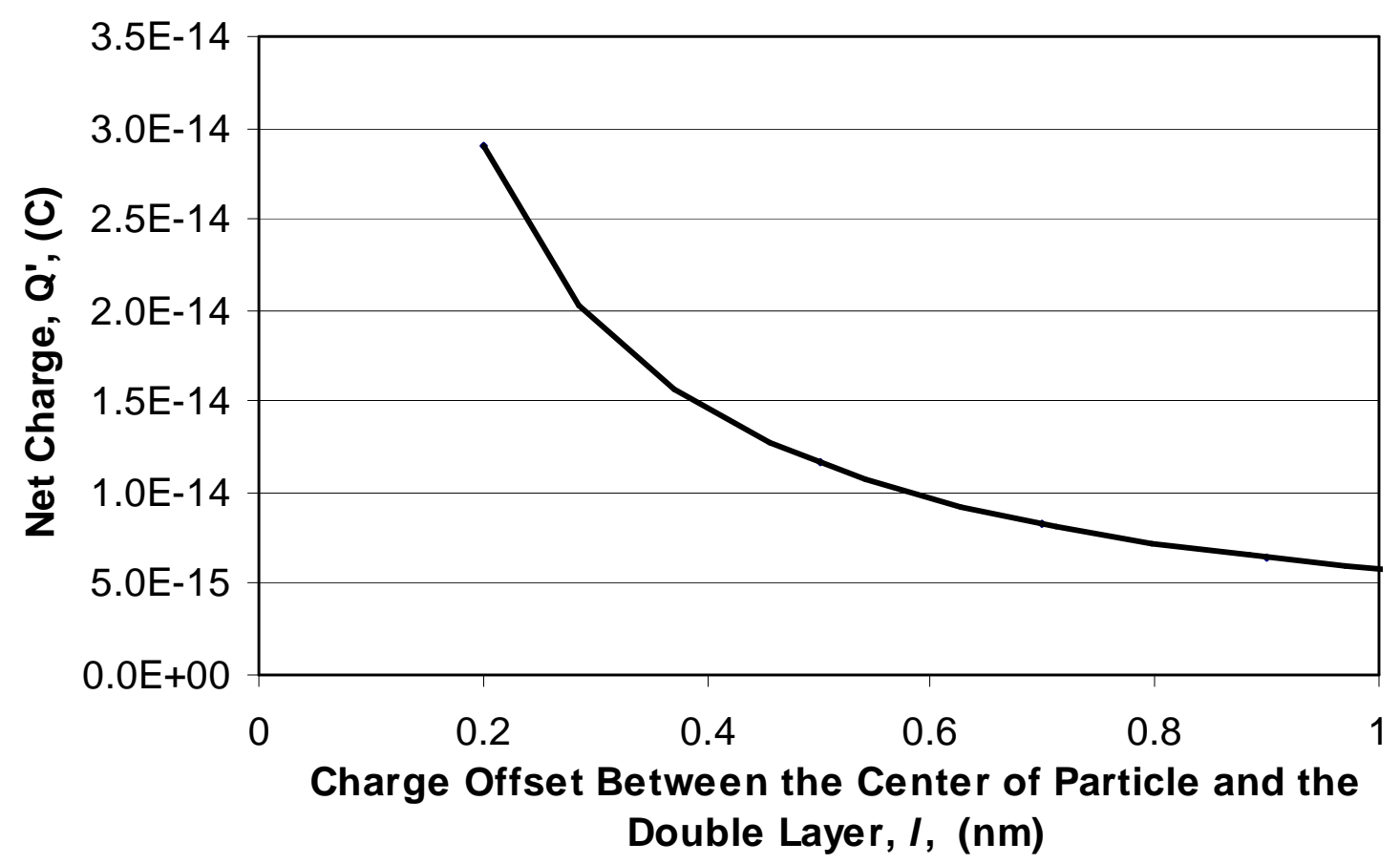

Figure 4.12: Different combinations of $Q^{\prime}$ and $l$ that will satisfy a fixed value of dipole moment, $m$

The force, $F$, computed for a fixed $m$ and different value of $l$, is shown in figure 4.13. At net charge displacements lower than $1 \mathrm{~nm}$, the removal force is comparable to the van der Waals adhesion force. These approximate calculations show that removal forces on the same order as adhesion forces can be produced in ionic solutions in conjunction with an acoustic field. 


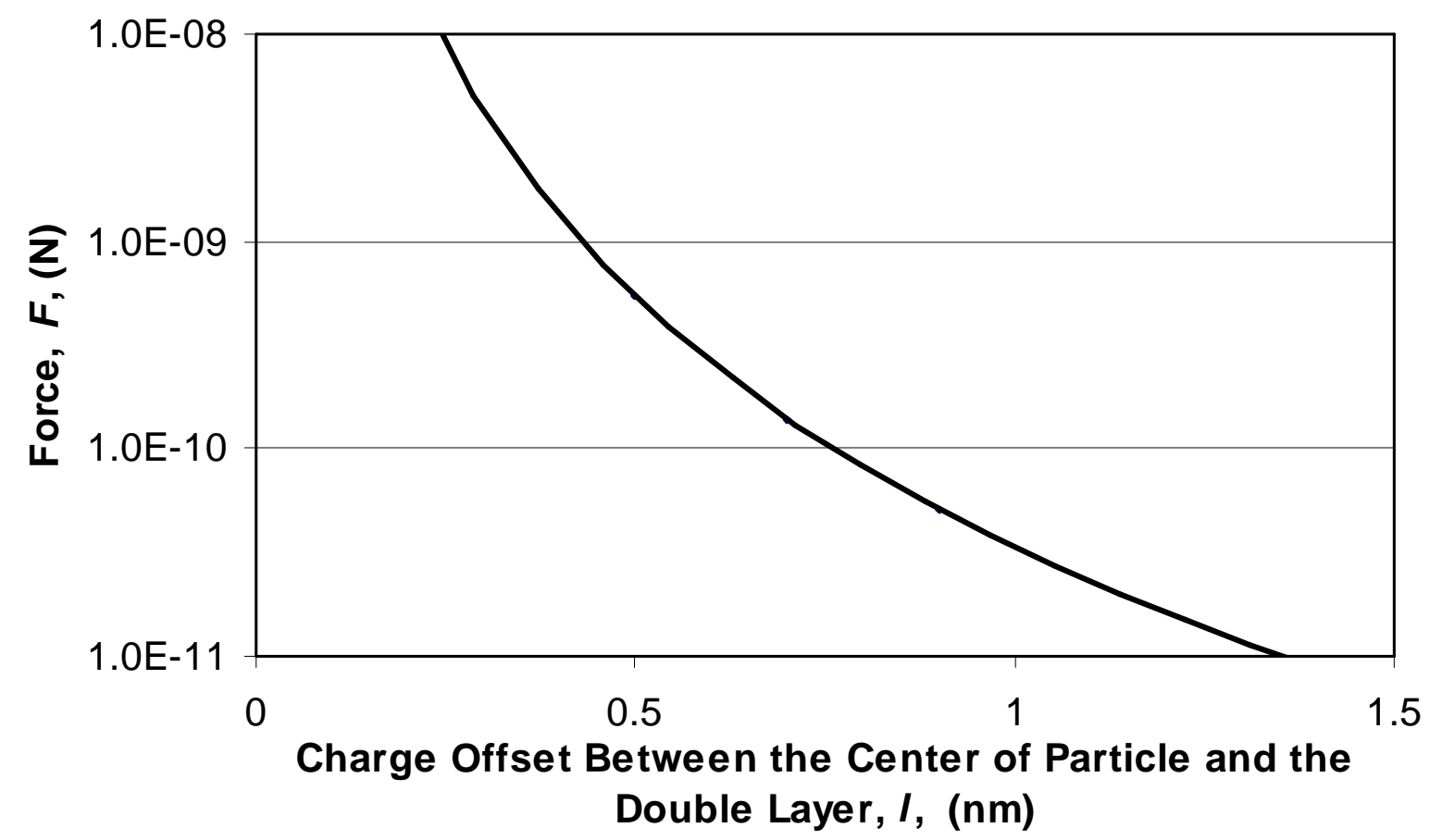

Figure 4.13: Magnitude of removal force, $F$, computed for fixed $m$ and different values of $l$, for transducer power density of $0.43 \mathrm{~W} / \mathrm{cm}^{2}$ and ionic strength of $3 \times 10^{-5} \mathrm{M}$

\subsubsection{Effect of Ionic Strength on Removal Force}

The effect of ionic strength on removal force can be understood from the dependence of removal force, $F$ on charge, $Q$ ' and charge offset, $l$. Assume a point center mass of charge $+Q$ surrounded by an ionic cloud of radius $r$ ' and charge $-Q$ as shown in figure 4.14 . 


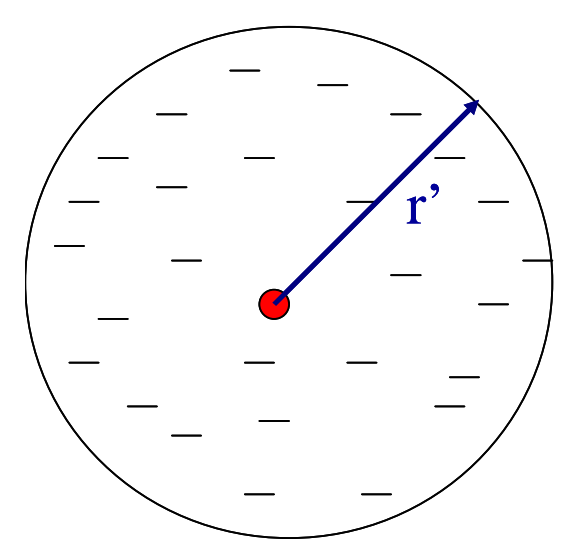

Figure 4.14: A point center mass of charge $+Q$ surrounded by ionic cloud of radius $r$ ' and charge $-Q$

In the absence of any acoustic field, the positive and negative charges are considered to act as though they are superimposed. The system has no dipole moment in this situation. When an acoustic field is applied, the center of the surrounding ionic charge becomes offset from its initial position by a distance $l$, which can be used to compute $Q^{\prime}$ and F' using equations 4.1 and 4.2 respectively.

$Q^{\prime}=Q\left(\frac{\frac{4}{3} \pi l^{3}}{\frac{4}{3} \pi r^{\prime 3}}\right)$

$F^{\prime}=\frac{Q^{\prime 2}}{l^{2}}=\frac{Q^{2} l^{4}}{r^{\prime 6}}$ 
One can notice from equation 4.1 that $Q^{\prime}$ becomes equal to $Q$ when $l$ attains a maximum achievable value of $r^{\prime}$ as shown in figure 4.15 .

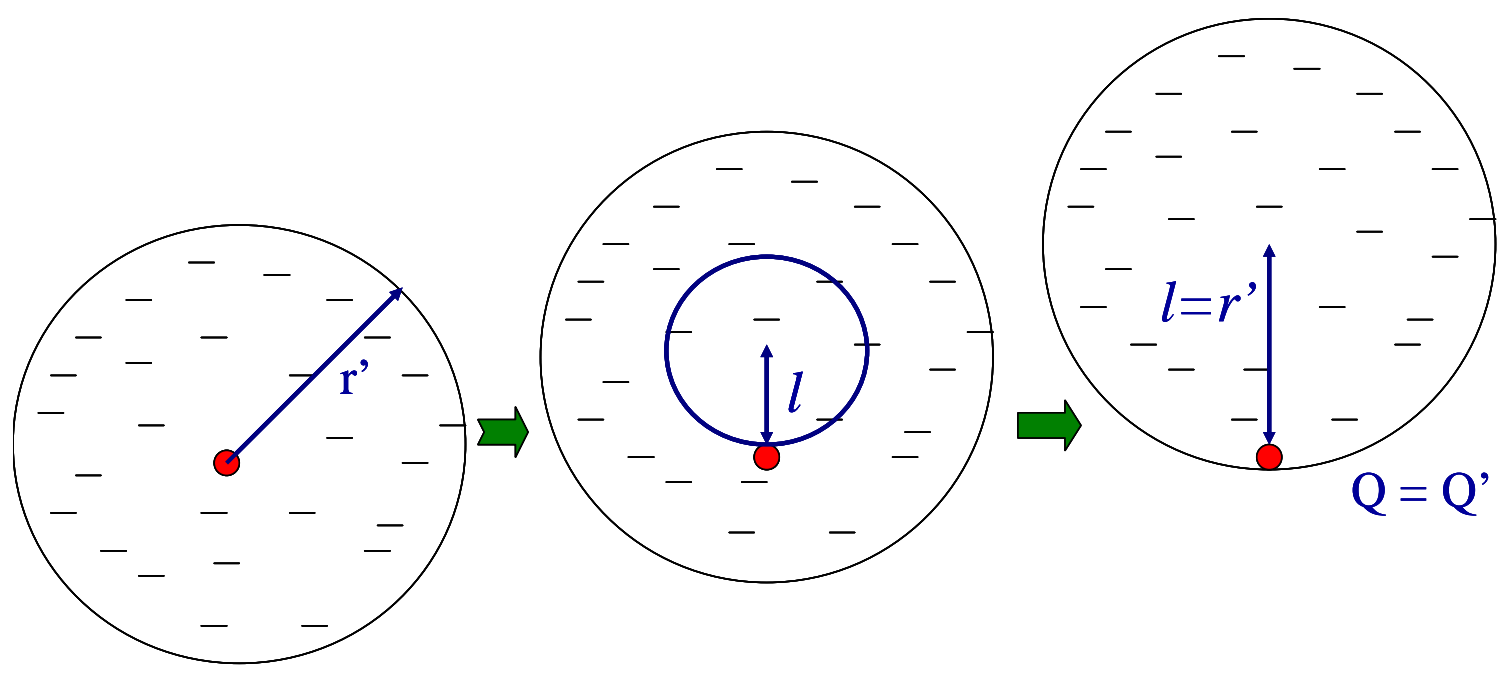

Figure 4.15: Shifting of ionic charge due to applied acoustic field

Now, if the outer ionic cloud is compressed, say, due to an increase in solution ionic strength as shown in figure 4.16, the force F" and charge Q" for the same offset distance $l$ become:

$$
Q^{\prime \prime}=Q\left(\frac{\frac{4}{3} \pi l^{3}}{\frac{4}{3} \pi r^{\prime \prime 3}}\right)
$$




$$
F^{\prime \prime}=\frac{Q^{\prime \prime 2}}{l^{2}}=\frac{Q^{2} l^{4}}{r^{\prime \prime}}
$$
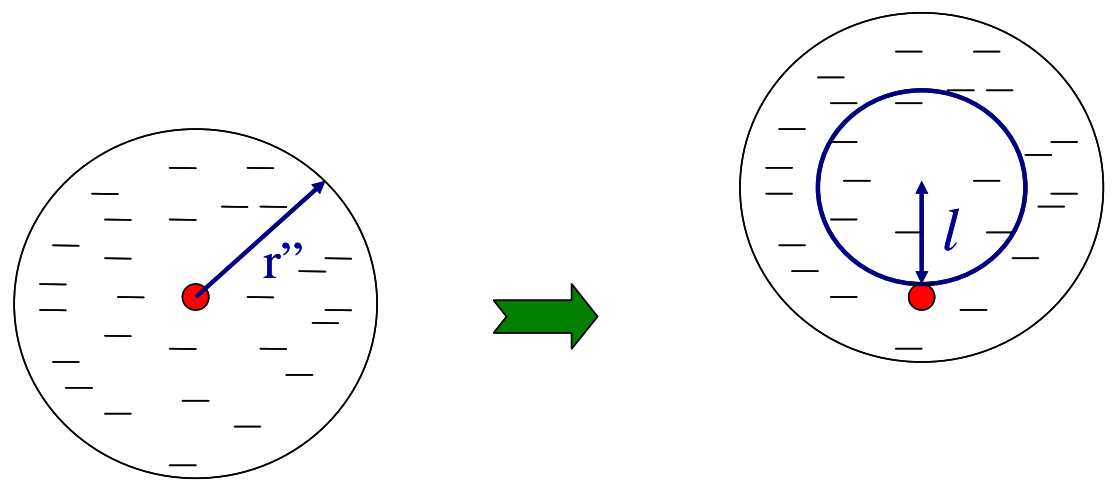

Figure 4.16: Shifting of ionic charge at higher ionic strength in an acoustic field

Since, r" is smaller than r', Q" will be higher than Q' for constant $l$ and F' will be greater than F'. However, with the compression of ionic cloud at higher ionic strength, the ions are distributed closer to the charged surface and therefore experience higher electrostatic attraction which can reduce the value of offset, $l$. Even for equivalent decrease in $l$ and $r$ ', F', will increase due to stronger dependency on $r$ ' than $l$. This suggests that the magnitude of removal force on the particle can increase with ionic strength.

In figure 4.17, the removal force is plotted as a function of charge offset, $l$, at two different solution ionic strengths of $0.0001 \mathrm{M}$ and $0.00003 \mathrm{M}$ and power densities of 0.43 
$\mathrm{W} / \mathrm{cm}^{2}$ and $0.15 \mathrm{~W} / \mathrm{cm}^{2}$. One can notice that a removal force of $1.5 \times 10^{-10} \mathrm{~N}$ can be obtained at two different offset $(l)$ values of $0.7 \AA$ and $0.5 \AA$. These offsets correspond to power densities and ionic strengths of $0.43 \mathrm{~W} / \mathrm{cm}^{2}$ and $3 \times 10^{-5} \mathrm{M}$ and $0.15 \mathrm{~W} / \mathrm{cm}^{2}$ and $10^{-4} \mathrm{M}$ respectively. Thus, one can employ either lower power density and higher ionic strength or vice versa to obtain equivalent values of removal force. By performing these calculations one can schematically obtain a plot between critical electrolyte concentration and power density as shown in figure 4.18 .

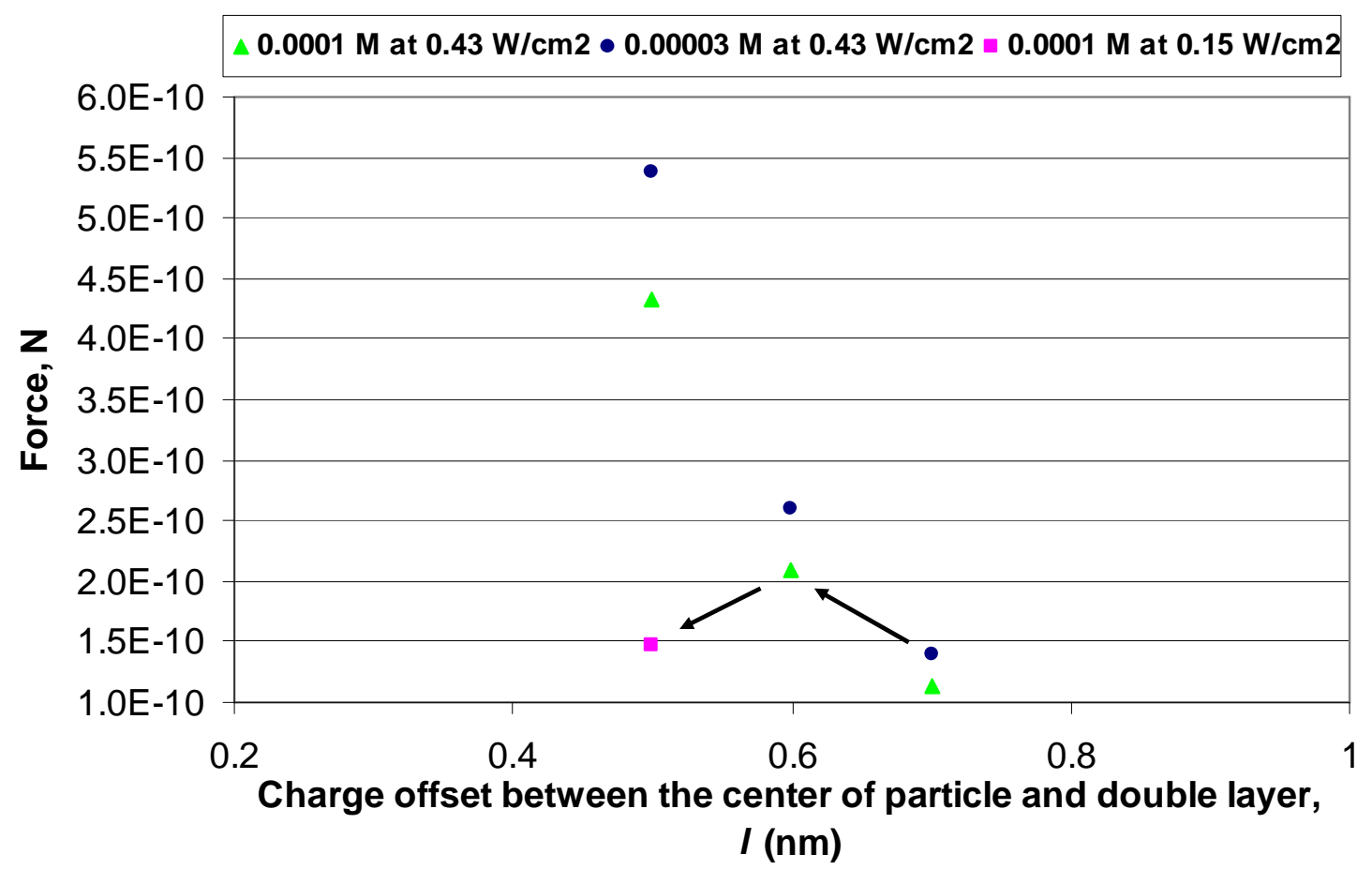

Figure 4.17: Removal force as a function of displacement at ionic strengths of $0.0001 \mathrm{M}$ and $0.00003 \mathrm{M}$ and power densities of $0.43 \mathrm{~W} / \mathrm{cm}^{2}$ and $0.15 \mathrm{~W} / \mathrm{cm}^{2}$ 


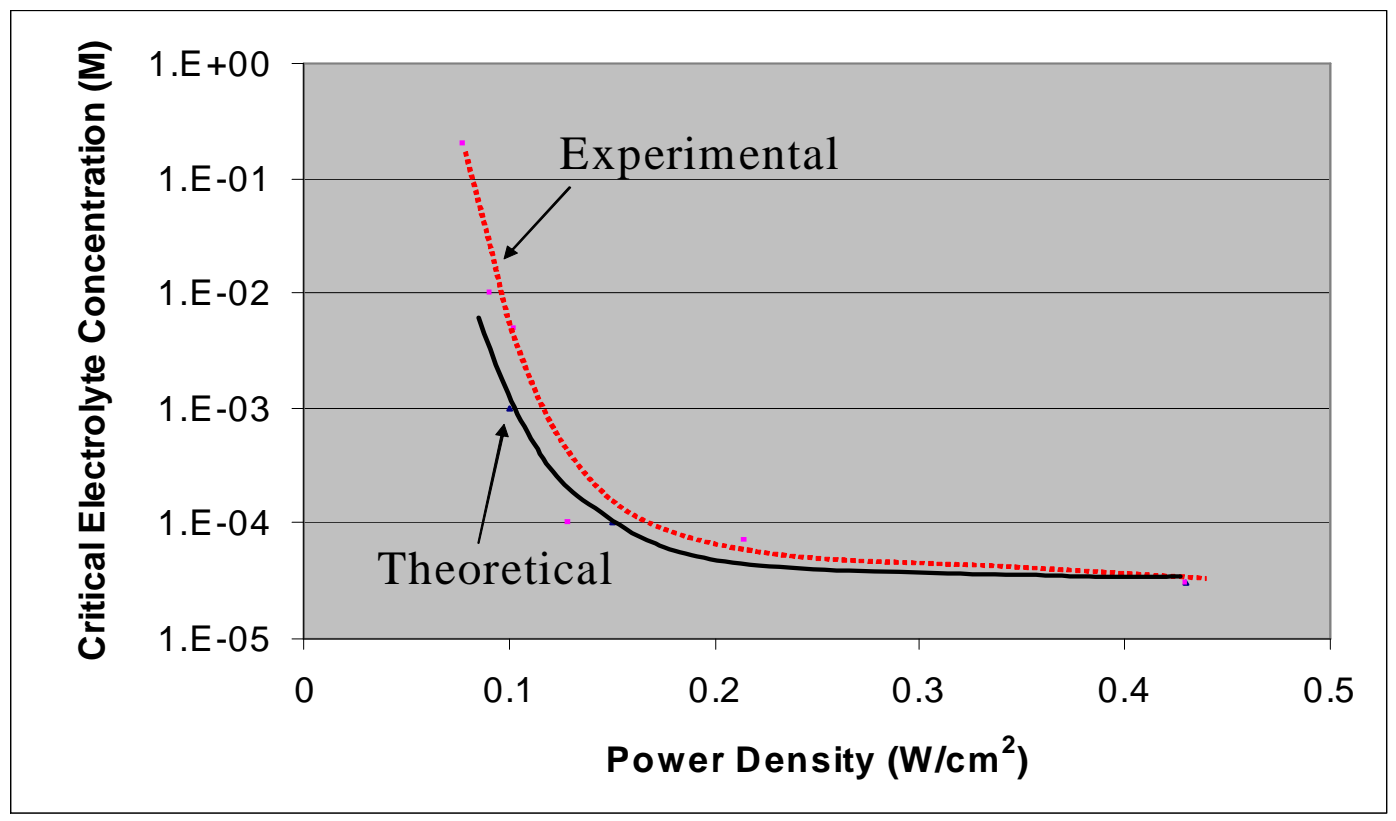

Figure 4.18: Critical electrolyte concentration as a function of power density

\subsubsection{Effect of Ionic Strength on Cavitation}

In this section, the effect of ionic strength on cavitation is discussed. The process of cavitation was quantified by two indirect means: 1) Measurement of size distribution of stable bubbles and 2) Sonoluminescence. The solution ionic strength was adjusted using $\mathrm{KCl}$.

\subsubsection{Modulation of Sound Wave Pressure Amplitude}

The pressure amplitude of the sound wave is related to solution properties such as bulk modulus and density by the equation 2.48 . In the case of aqueous $\mathrm{KCl}$ solution, the 
density and bulk modulus of the solution increase with the electrolyte concentration [4.1]. This is expected to increase the sound wave pressure amplitude as indicated in figure 4.20. As one may notice, the increase in pressure amplitude with $\mathrm{KCl}$ concentration is small. However, its effect on cavitation can be considerable. For example, the threshold for stable cavitation decreases rapidly close to the resonant bubble size as indicated by the stable cavitation threshold curve in figure 2.16 .

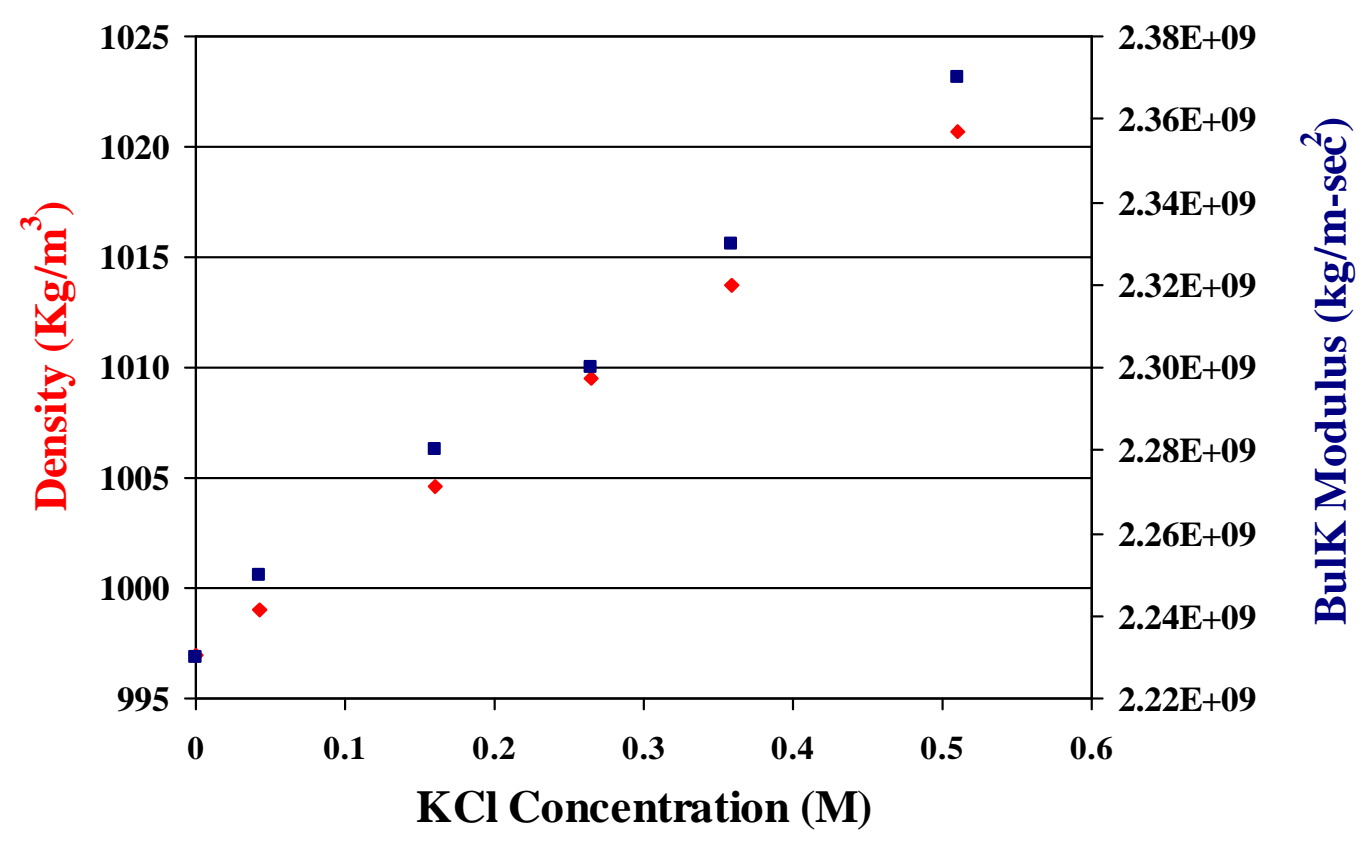

Figure 4.19: Bulk modulus and density of solution at different $\mathrm{KCl}$ concentration at $25{ }^{\circ} \mathrm{C}$ 


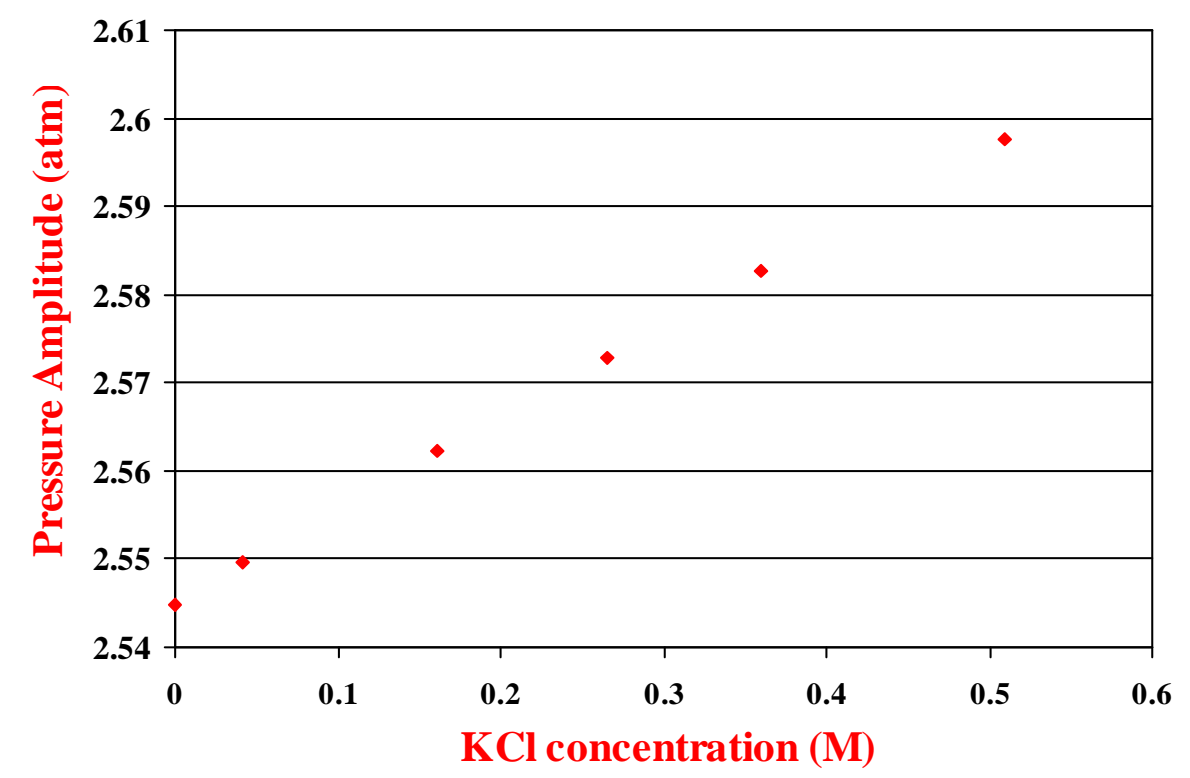

Figure 4.20: Sound wave pressure amplitude in $\mathrm{KCl}$ solution of different concentration at power density of $2.17 \mathrm{~W} / \mathrm{cm}^{2}$ and solution temperature of $25{ }^{0} \mathrm{C}$

4.2.2.2. Experimental Investigation of Higher Concentrations of Potassium Chloride on Sound Wave Pressure Amplitude and Bubble Size Distribution

In order to experimentally determine the effect of $\mathrm{KCl}$ concentration on sound wave pressure amplitude, measurements were made using a hydrophone. The root mean square voltage, $\mathrm{V}_{\mathrm{RMS}}$, an indicator of pressure amplitude, was measured in $\mathrm{KCl}$ solutions of different concentrations. The results, shown in figure 4.21, demonstrate that the addition of $\mathrm{KCl}$ to DI water increases the pressure amplitude of the sound wave. The $\mathrm{V}_{\mathrm{RMS}}$ values in DI water corresponded to a maximum pressure amplitude of approximately $1.66 \mathrm{~atm}$ and $2.33 \mathrm{~atm}$ at $0.43 \mathrm{~W} / \mathrm{cm}^{2}$ and $2.17 \mathrm{~W} / \mathrm{cm}^{2}$ respectively as measured using a calibrated HNR-1000 Onda Corporation hydrophone. The increase in 
pressure amplitude with the addition of $\mathrm{KCl}$ does not begin until a $\mathrm{KCl}$ concentration of about $0.1 \mathrm{M}$ is reached. At $\mathrm{KCl}$ concentrations lower than $0.1 \mathrm{M}$, it is possible that the bulk modulus and density of the $\mathrm{KCl}$ solution do not increase considerably so as to cause an increase in sound wave pressure amplitude. The difference in pressure amplitude between $\mathrm{KCl}$ solution and DI water also increases as the power density increases.

It may be noted that the theoretical calculations for pressure amplitude performed earlier did not include any cavitation effects in the solution. On the other hand, actual experimental conditions involve scattering of sound waves from oscillations of stable cavities (microstreaming) which will affect $\mathrm{V}_{\mathrm{RMS}}$ values. 

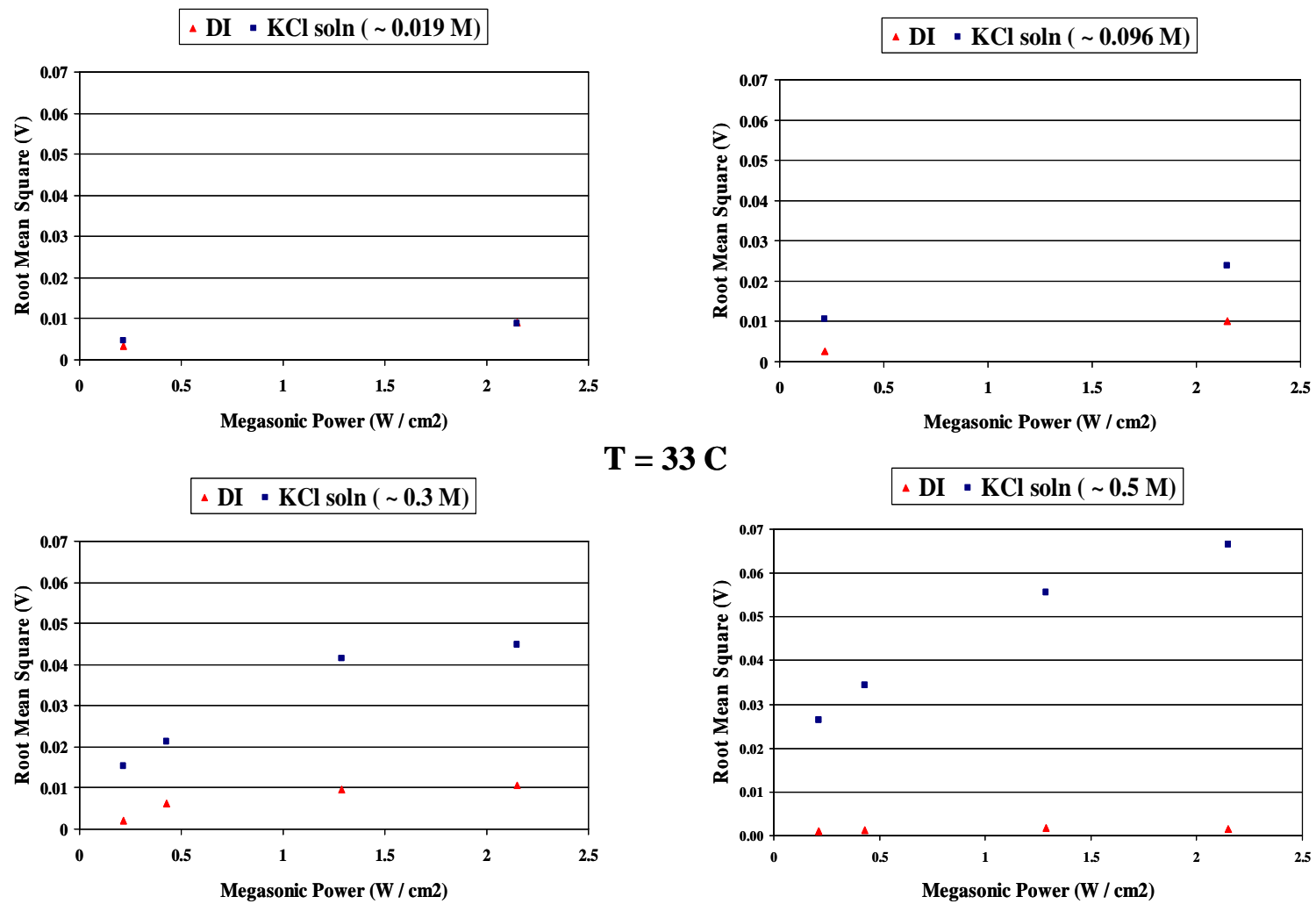

$\mathbf{T}=\mathbf{3 3} \mathbf{C}$

$\triangle \mathrm{DI} \cdot \mathrm{KCl} \operatorname{soln}(\sim 0.5 \mathrm{M})$

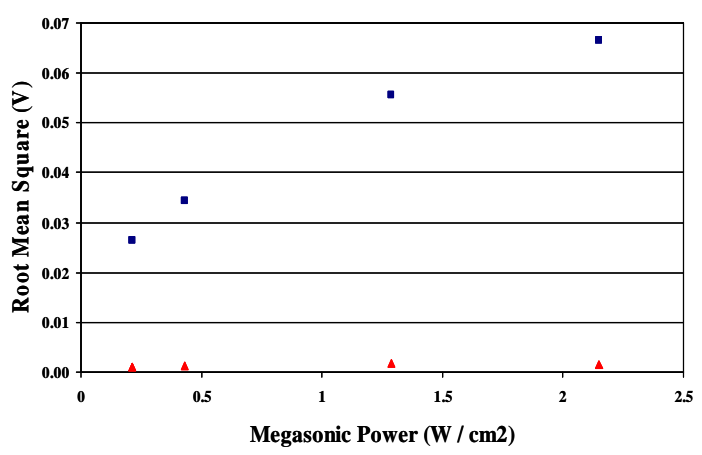

Figure 4.21: Hydrophone measurements in $\mathrm{KCl}$ solutions of different concentration and power densities

Fast Fourier Transform (FFT) of the pressure amplitude data was performed to obtain the distribution of frequencies of oscillating bubbles in solutions. Assuming the bubbles to be air bubbles, and assuming relative changes in bubble volume due to bubble expansion and contraction in response to the pressure variations to be small, equation 2.57 from linear theory of small oscillations of a gas bubble was used to obtain the bubble size distribution. 
The bubble size distribution in $\mathrm{KCl}$ solutions of different concentrations irradiated at $2.17 \mathrm{~W} / \mathrm{cm}^{2}$ is shown in figure 4.22 . Since transient bubbles exist for less than one cycle, this bubble size distribution refers to stable bubbles in the system. It is clear from figure 4.22 that increasing $\mathrm{KCl}$ concentration increases the bubble sizes and also widens the distribution. At $0.02 \mathrm{M} \mathrm{KCl}$ solution, the bubble sizes span mainly from 1 to 10 microns. Bubbles of larger sizes start emerging with the further addition of $\mathrm{KCl}$. At $0.5 \mathrm{M}$ $\mathrm{KCl}$, a significant population of bubbles exists in the entire range of sizes from 0.1 to 100 $\mu \mathrm{m}$. The stable bubbles often transform into transient cavities depending on the solution properties and acoustic pressure amplitude and frequency.

\subsection{Prediction of Transient Cavities from Bubble Size Distribution}

Based on the bubbles sizes and sound wave frequency, a pressure threshold exists for rectified diffusion, lower transient cavitation and transient cavitation as predicted by Apfel and Walton for air saturated water in the frequency range from $20 \mathrm{kHz}$ to $2.5 \mathrm{MHz}$ $[2.53,2.62 \& 4.2]$. As discussed earlier, the pressure amplitude of the sound wave ranges from $2.53 \mathrm{~atm}$ in DI water to $2.60 \mathrm{~atm}$ in $0.5 \mathrm{M} \mathrm{KCl}$ solution at a power density of 2.17 $\mathrm{W} / \mathrm{cm}^{2}$ and solution temperature of $25{ }^{\circ} \mathrm{C}$. In this pressure amplitude range, bubbles of sizes lower than $6 \times 10^{-7} \mathrm{~m}$ will fall in the stable threshold region and will dissolve away or become stabilized at some smaller size. In the region between the stable and transient thresholds, bubbles can grow in the sound field and may eventually reach the transient threshold. At $0.5 \mathrm{M} \mathrm{KCl}$ concentration, bubbles of sizes greater than $2 \times 10^{-6} \mathrm{~m}$ will reach 
the region above the transient threshold and will immediately become active as transient cavities, by expanding and eventually exploding. Bubbles of size greater than $10^{-3} \mathrm{~m}$ will be removed from the system due to buoyancy. Since both pressure amplitude of the sound wave and stable bubble size distribution increase with $\mathrm{KCl}$ concentration, it is likely that higher pressure amplitude leads to the formation of bigger cavities.

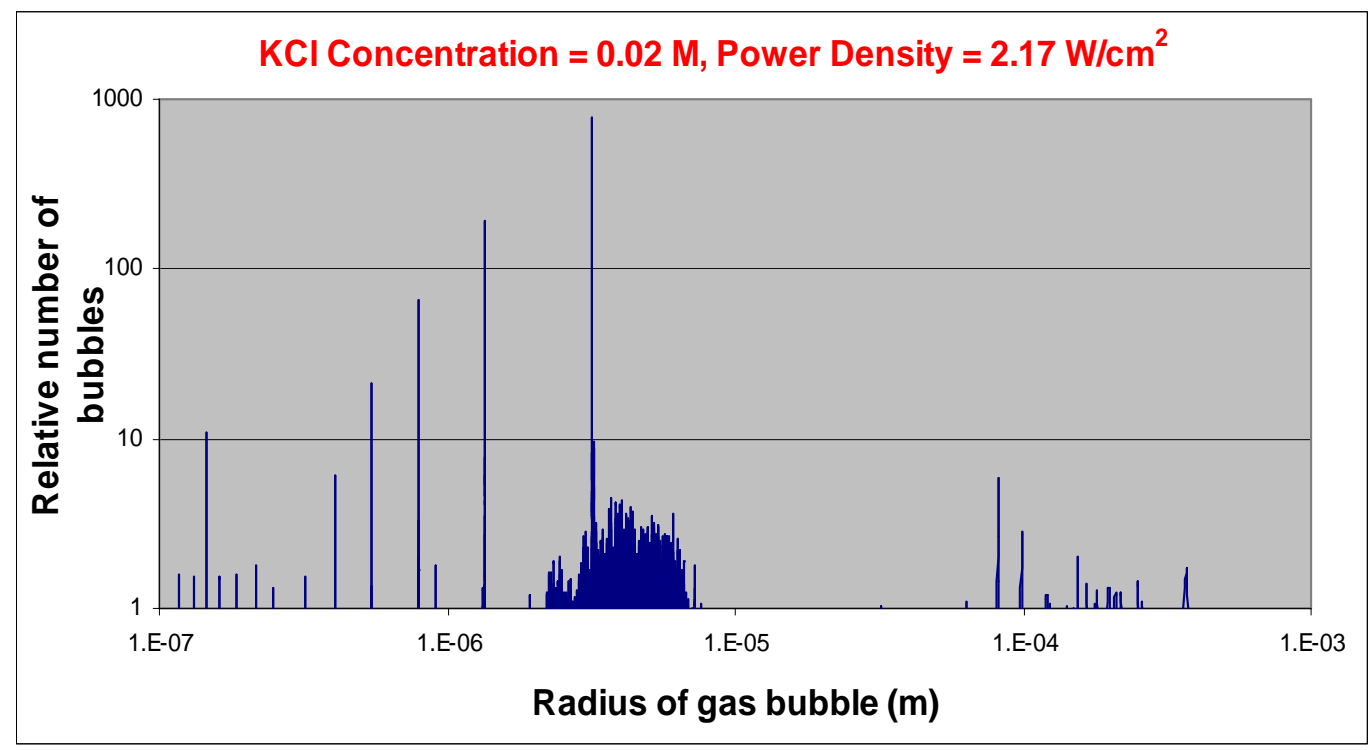



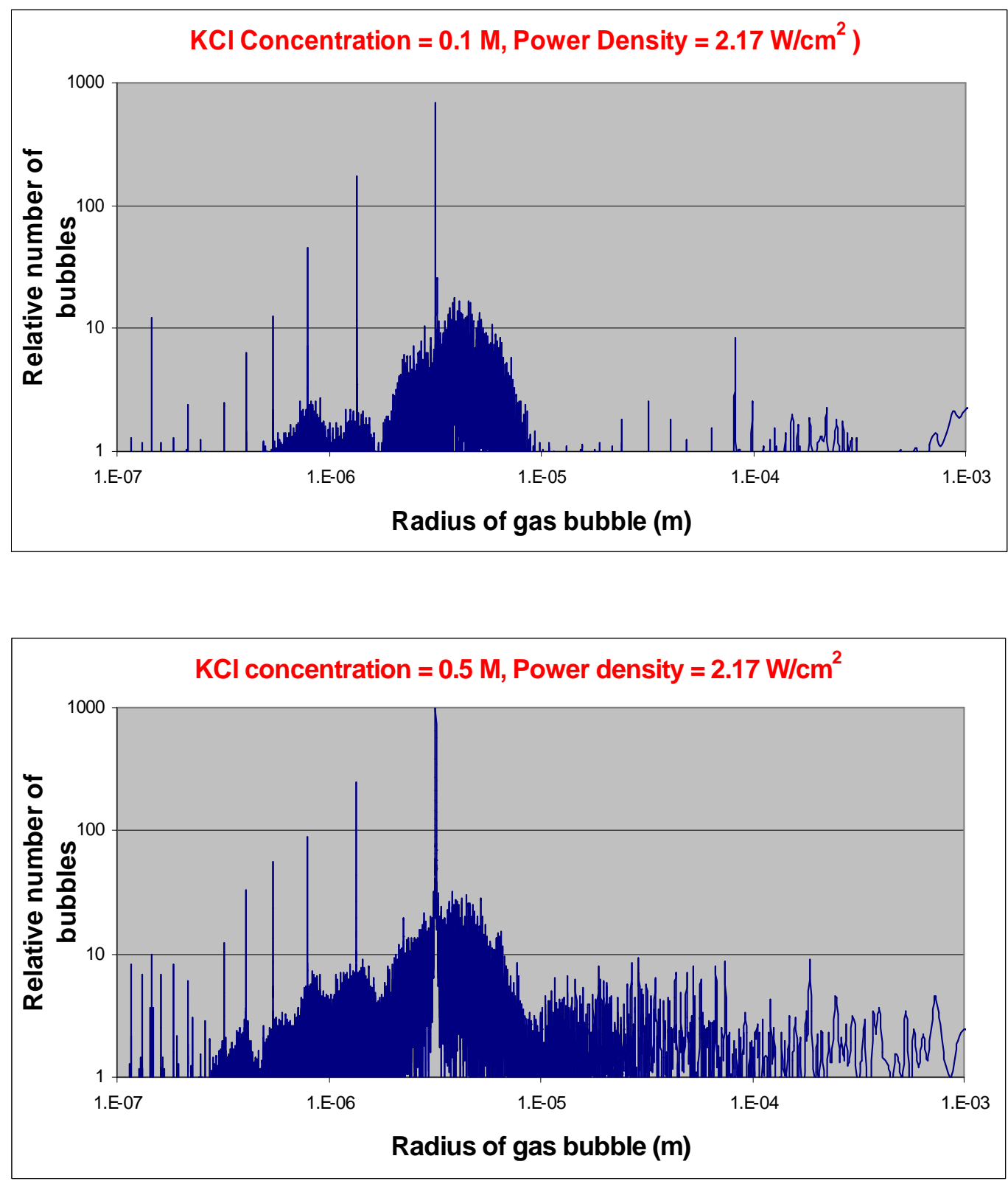

Figure 4.22: Bubble size distribution in $\mathrm{KCl}$ solution of different concentrations at $30{ }^{0} \mathrm{C}$ and power density of $2.17 \mathrm{~W} / \mathrm{cm}^{2}$ 


\subsubsection{Effect of Pressure Amplitude on Sonoluminescence}

In order to examine the effect of pressure amplitude on cavitation intensity, SL was measured in $\mathrm{DI}$ water and $\mathrm{KCl}$ solutions of different concentration using the cavitation probe. Figure 4.23 shows the photon count rate as a function of number of occurrences in DI water and $\mathrm{KCl}$ solutions irradiated with a megasonic power density of $2.17 \mathrm{~W} / \mathrm{cm}^{2}$ for a period of 10 minutes at $30{ }^{\circ} \mathrm{C}$. In DI water, the photon count rate did not exceed $9 \times 10^{5}$ counts/s. By contrast, the photon count rate as high as $2 \times 10^{6}$ counts/s was observed in $\mathrm{KCl}$ solutions of concentrations $0.6 \mathrm{M}$ and $1.1 \mathrm{M}$, suggesting that $\mathrm{KCl}$ solutions exhibit more intense cavitation than DI water. In the region of higher photon count rate (greater than $8 \times 10^{5}$ counts/s), a $1.1 \mathrm{M} \mathrm{KCl}$ solution showed greater occurrence than $0.6 \mathrm{M} \mathrm{KCl}$ followed by DI water. The average photon count rate of $\mathrm{KCl}$ solutions was approximately $80 \%$ higher than that in DI water. Thus, it appears that the formation and collapse of bigger bubbles in $\mathrm{KCl}$ solutions leads to more intense cavitation compared to DI water. 


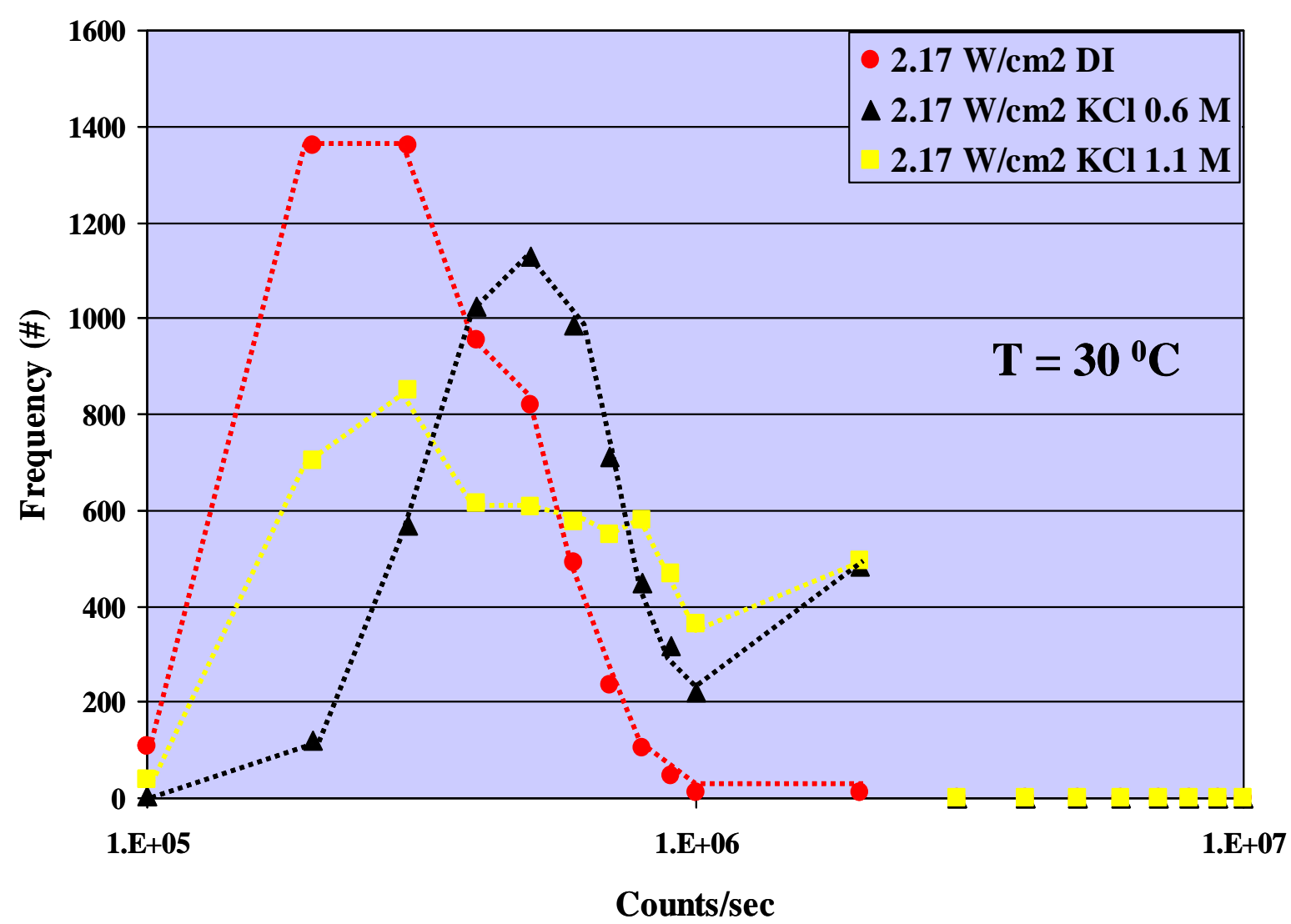

Figure 4.23: Cavitation in DI water and $\mathrm{KCl}$ solutions of two different concentrations for $10 \mathrm{~min}$ at $30{ }^{\circ} \mathrm{C}$ and power density of $2.17 \mathrm{~W} / \mathrm{cm}^{2}$

4.2.3. Effect of Pressure Amplitude on Removal of Silica Particles in Higher Concentrations of Potassium Chloride Solutions

Previous studies on pressure amplitude and cavitation measurements showed evidence of bigger and wider distribution of stable bubbles and more powerful cavitation in $\mathrm{KCl}$ solutions in comparison to DI water. Scattering of the sound waves from the stable bubbles, especially resonating bubbles, can generate rapid currents in localized 
regions by a process known as microstreaming, as discussed earlier in section 2.4.5.2. These currents can provide sufficient convection to transport the dislodged particles away from a wafer surface [1.45]. In addition, stable cavities often transform into transient cavities. The violent collapse of these transient cavities can lead to the formation of shock waves and fluid jet formation that have been found to be extremely useful in dislodging particles from surfaces. Potassium chloride solutions of concentrations greater than $10^{-2} \mathrm{M}$ have shown to exhibit higher cavitation than DI water and therefore can be expected to provide additional force for removal of particles from surfaces.

In explaining the removal of silica particles from wafer surfaces during megasonic cleaning in potassium chloride solutions, the electro-acoustic force due to CVP was considered as a dominant removal force. Cavitation effects were not taken into account. In examining the data in figure 4.4, where removal of silica particles was investigated as a function of $\mathrm{KCl}$ concentration at different power densities, although electro-acoustic forces are expected to be dominant at lower concentrations of $\mathrm{KCl}$, the contribution of forces from cavitation cannot be ignored at a concentration greater than $10^{-2} \mathrm{M}$. For example, in the case of aminated silica particles, the increase in PRE from 20 to $75 \%$ with an increase in $\mathrm{KCl}$ concentration from $10^{-6}$ to $10^{-2} \mathrm{M}$ at $0.43 \mathrm{~W} / \mathrm{cm}^{2}$ may be due to the electro-acoustic effect alone. A further increase in PRE to $95 \%$ in $0.5 \mathrm{M} \mathrm{KCl}$ can be expected to be a combined effect from electro-acoustic and cavitation forces. Similarly, the removal of aminated silica particles at lower power density of $0.077 \mathrm{~W} / \mathrm{cm}^{2}$ does not occur until a $\mathrm{KCl}$ concentrations greater than $0.01 \mathrm{M}$ is reached. Since cavitation 
increases in $\mathrm{KCl}$ solutions with concentration greater than $0.01 \mathrm{M}$, the removal of particles can be expected to get affected due to this effect. Thus, both cavitation and electro-acoustic forces play a significant role in improving removal efficiency of silica particles from wafer surfaces.

\subsubsection{Effect of Temperature on Removal of Aminated Silica Particles}

Effect of thermal energy on removal of aminated silica particles in $\mathrm{KCl}$ solutions was investigated at two different solution temperatures, $30{ }^{0} \mathrm{C}$ and $40{ }^{0} \mathrm{C}$. The experimental conditions were chosen such that PRE values at $30{ }^{0} \mathrm{C}$ were about $50 \%$, where comparison with PRE values at a higher temperature was possible. As shown in table 4.1, PRE increases with temperature in $\mathrm{KCl}$ solution at a particular concentration and power density. In $10^{-4} \mathrm{M} \mathrm{KCl}$ solution, at a power density of $0.129 \mathrm{~W} / \mathrm{cm}^{2}, \mathrm{PRE}$ increases from $\sim 50 \%$ to $80 \%$ when the temperature is increased from 30 to $40{ }^{\circ} \mathrm{C}$. At a higher $\mathrm{KCl}$ concentration of $0.3 \mathrm{M}$ and a lower power density of $0.077 \mathrm{~W} / \mathrm{cm}^{2}, \mathrm{PRE}$ increases from 65 to $90 \%$.

In explaining the data on the effect of electrolyte concentration in section 4.2.3., it was stated that at ionic strengths lower than $0.01 \mathrm{M}$, the electro-acoustic effect appears to play a role in the removal of particles where as at higher ionic strengths both cavitation and electro-acoustic effects were important. The effect of temperature on PRE at low electrolyte concentration can be explained as follows. The removal force, given by 
equation 2.41, depends on the magnitude of the dipole moment. The temperature dependent variables in the dipole moment equation (equation 2.39) include zeta potential of the particle and the dielectric constant of the electrolyte solution.

Table 4.1: Effect of temperature on removal of aminated silica particles from wafer surface in $\mathrm{KCl}$ solutions of different concentrations and power densities

\begin{tabular}{|c|c|c|c|c|}
\hline $\begin{array}{c}\text { Experimental } \\
\text { Conditions }\end{array}$ & $\begin{array}{c}\text { Avg } \\
\text { PRE \% } \\
\left(40^{0} \mathrm{C}\right)\end{array}$ & $\begin{array}{c}\text { Avg } \\
\text { PRE \% } \\
\left(30^{\circ} \mathrm{C}\right)\end{array}$ & $\begin{array}{c}\text { Std } \\
\text { dev \% } \\
\left(40^{\circ} \mathrm{C}\right)\end{array}$ & $\begin{array}{c}\text { Std } \\
\text { Dev \% } \\
\left(30^{\circ} \mathrm{C}\right)\end{array}$ \\
\hline $0.129 \mathrm{~W} / \mathrm{cm}^{2}, 10^{-4} \mathrm{M}$ & 80 & 55 & 9 & 1 \\
\hline $0.103 \mathrm{~W} / \mathrm{cm}^{2}, 10^{-3} \mathrm{M}$ & 80 & 40 & 6 & 2 \\
\hline $0.103 \mathrm{~W} / \mathrm{cm}^{2}, 10^{-2} \mathrm{M}$ & 80 & 60 & 1 & 6 \\
\hline $0.077 \mathrm{~W} / \mathrm{cm}^{2}, 0.1 \mathrm{M}$ & 65 & 25 & 4 & 11 \\
\hline $0.077 \mathrm{~W} / \mathrm{cm}^{2}, 0.3 \mathrm{M}$ & 90 & 65 & & 2 \\
\hline
\end{tabular}

A theoretical relation between $\mathrm{ZP}$ and temperature for 1:1 electrolyte can be obtained from equation $2.17 \mathrm{~b}$ as:

$\psi_{Z P}=\frac{2 k_{B} T}{e} \sinh ^{-1}\left(\frac{\sigma_{\text {shear Plane }}}{\left(8 \varepsilon_{0} D k_{B} T n_{\infty}\right)^{1 / 2}}\right)$

where $\psi_{Z P}$ is the zeta potential and $\sigma_{\text {shear Plane }}$ is the charge density at the shear plane. 
Increasing the temperature has two effects on $\psi_{Z P}$.Obviously $\frac{2 k_{B} T}{e}$ increases with temperature; between 30 and $40{ }^{\circ} \mathrm{C}$ it increases at the rate of $0.33 \%$ per degree $\mathrm{C}$. The effect of the second term $\sinh ^{-1}\left(\frac{\sigma_{\text {shear Plane }}}{\left(8 \varepsilon_{0} D k_{B} T n_{\infty}\right)^{1 / 2}}\right)$ on temperature is less obvious. In the case of aminated silica particles, $\sigma_{\text {shear Plane }}$ will depend on the equilibrium constant of the following reaction:

$-\mathrm{NH}_{2}+\mathrm{H}^{+} \longrightarrow-\mathrm{NH}_{3}^{+}$

The equilibrium constant changes with temperature and can be determined if the Gibbs free energy of reaction is known for reaction $\mathrm{R} 4.1$.

The variation of the dielectric constant $(D)$ of water with temperature $(T$ in $\mathrm{K})$ at atmospheric pressure is given by equation 4.6 [4.3]. The dielectric constant decreases by approximately $4 \%$ with an increase in temperature from $30{ }^{\circ} \mathrm{C}$ to $40{ }^{0} \mathrm{C}$.

$D=5321 T^{-1}+233.76-0.9297 T+0.1417 * 10^{-2} T^{2}-0.8292 * 10^{-6} T^{3}$

Revil, et al., have shown experimentally that the ZP of silica at $\mathrm{pH}$ of 7.0 would increase linearly with temperature at the rate of $1.9 \%$ per degree $\mathrm{C}$ at temperatures lower than $100{ }^{0} \mathrm{C}$ in solution of ionic strength $10^{-3} \mathrm{M}[4.4]$. These results were consistent with 
experimental findings of Somasundaran, et al., and Ishido, et.al., [4.5, 4.6]. If one assumes that the ZP increases by approximately $20 \%$ (as reported in literature) and dielectric constant of water decreases by $4 \%$ with 10 degree rise in temperature, the overall effect will be an increase in dipole moment with an increase in temperature. Although the increase in dipole moment is not expected to be large, it will result in an additional removal force that can improve the cleaning efficiency. It is important to mention here that the electro-acoustic force due to IVP was not considered in these calculations of the temperature effect on removal force. Although the ionic vibration potential will increase due to increases in the difference in mobilities of $\mathrm{K}^{+}$and $\mathrm{Cl}^{-}$ions with temperature as shown in figure 4.24, the force will remain orders of magnitude lower than the adhesion force and hence will not affect the removal efficiency of particles.

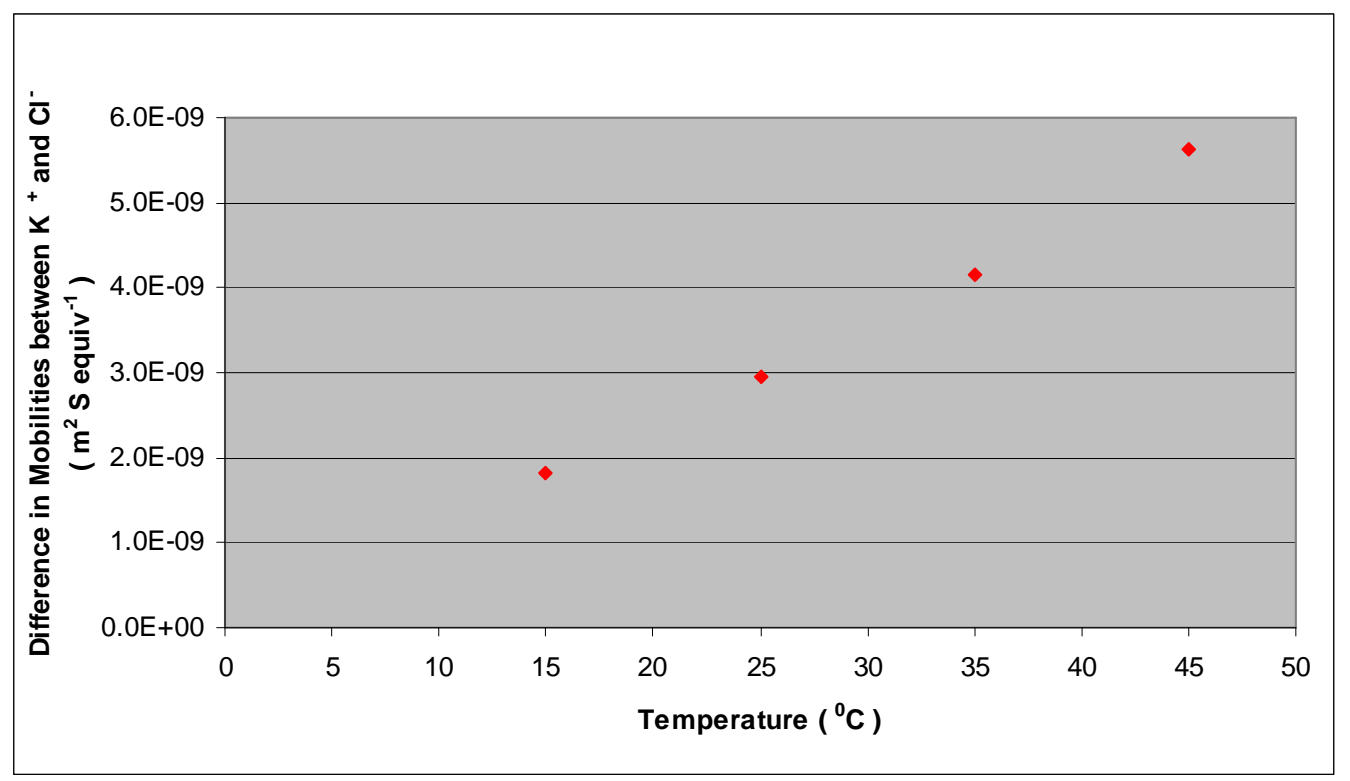

Figure 4.24: Effect of temperature on mobilities of $\mathrm{K}^{+}$and $\mathrm{Cl}^{-}$ions in $\mathrm{KCl}$ solution of infinite dilution [4.7] 
As discussed earlier, removal of silica particles at higher ionic strengths (greater than $0.01 \mathrm{M}$ ) can be expected to be affected by cavitation. Since PRE was observed to increase with temperature in $0.1 \mathrm{M}$ and $0.3 \mathrm{M} \mathrm{KCl}$ solutions at a power density of 0.077 $\mathrm{W} / \mathrm{cm}^{2}$, the effect of temperature on cavitation was initially investigated for these conditions. It was found that at this power density, the sensitivity of cavitation probe was low with significant scatter in the data. Hence, to study the effect of temperature on cavitation further, experiments were conducted at a higher power density of $0.43 \mathrm{~W} / \mathrm{cm}^{2}$. Measurements of SL were made in $0.3 \mathrm{M} \mathrm{KCl}$ solutions at temperatures of $30{ }^{\circ} \mathrm{C}$ and $40{ }^{0} \mathrm{C}$ for 10 minutes. Figure 4.25 shows the photon count rate plotted as a function of number of occurrences for the two temperature conditions. At a lower temperature of 30 ${ }^{0} \mathrm{C}$, the photon count rate in $0.3 \mathrm{M} \mathrm{KCl}$ solution did not exceed $2 \times 10^{4}$ counts/s. By contrast, a photon count rate as high as $3 \times 10^{5}$ counts/s was observed in a solution maintained at $40{ }^{\circ} \mathrm{C}$, suggesting that $\mathrm{KCl}$ solution at higher temperatures exhibits more intense cavitation than that at lower temperatures. The average photon count rate in $\mathrm{KCl}$ solution at $40{ }^{0} \mathrm{C}$ was approximately 40 times higher than the one at $30{ }^{\circ} \mathrm{C}$. Thus, PRE can be expected to increase with temperature in $\mathrm{KCl}$ solutions of higher concentration due to increase in cavitation.

Temperature can have an effect on several solution variables which can influence cavitation. An increase in temperature increases the solution vapor pressure and decreases the surface tension. The increase in vapor pressure reduces the speed of the collapsing bubble and is expected to reduce cavitation (see section 2.4.4). On the other hand, the 
speed of the collapsing bubble is known to increase with decrease in solution surface tension, which reduces the intensity of collapse (see section 2.4.3.4). Thus, an increase in vapor pressure and decrease in surface tension have opposing effects on cavitation. Further, the dissolution of gases decreases with an increase in temperature. A higher amount of dissolved gases provides a cushioning effect to the collapse of bubbles and reduces the intensity of SL. Due to the complex nature of the problem, the reason for increase in cavitation with temperature in $\mathrm{KCl}$ solution is not clear.

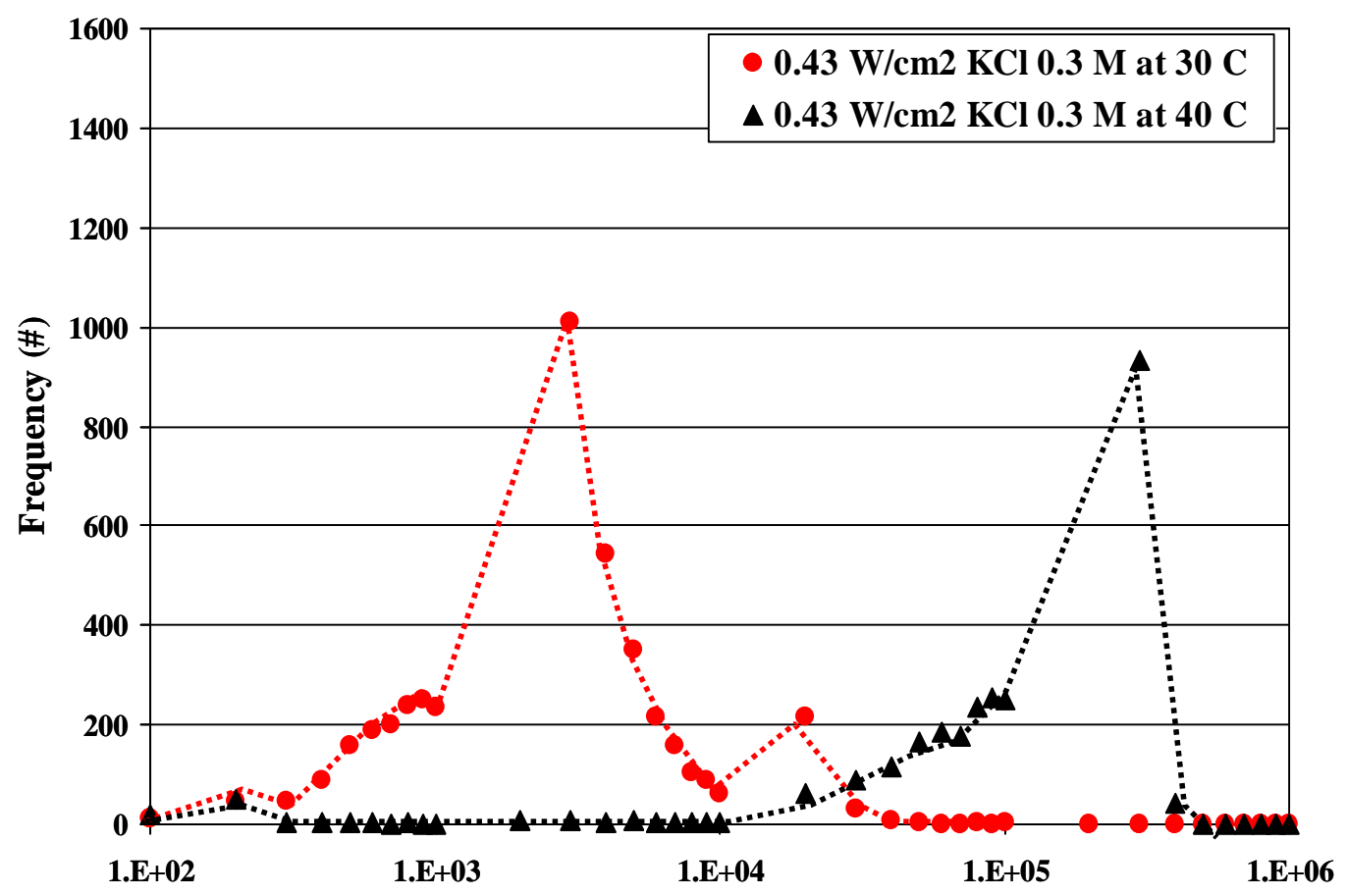

Counts/sec

Figure 4.25: $\mathrm{SL}$ in $0.3 \mathrm{M} \mathrm{KCl}$ solution at power density of $0.43 \mathrm{~W} / \mathrm{cm}^{2}$ and temperatures of $30{ }^{\circ} \mathrm{C}$ and $40{ }^{\circ} \mathrm{C}$ for $10 \mathrm{~min}$ 


\subsubsection{Effect of Solution $\mathrm{pH}$ on Removal of Aminated Silica Particles}

The removal of aminated silica particles from wafer surfaces was examined in solutions of different $\mathrm{pH}$ and equivalent ionic strengths at a power density of $0.43 \mathrm{~W} / \mathrm{cm}^{2}$ and a temperature of $30{ }^{\circ} \mathrm{C}$. The alkaline or acidic $\mathrm{pH}$ conditions were adjusted using sodium hydroxide/ammonium hydroxide or hydrochloric acid respectively. The $\mathrm{pH}$ values at different ionic strengths for $\mathrm{HCl}, \mathrm{NaOH}, \mathrm{NH}_{4} \mathrm{OH}$ and $\mathrm{KCl}$ are displayed in figure 4.26 .

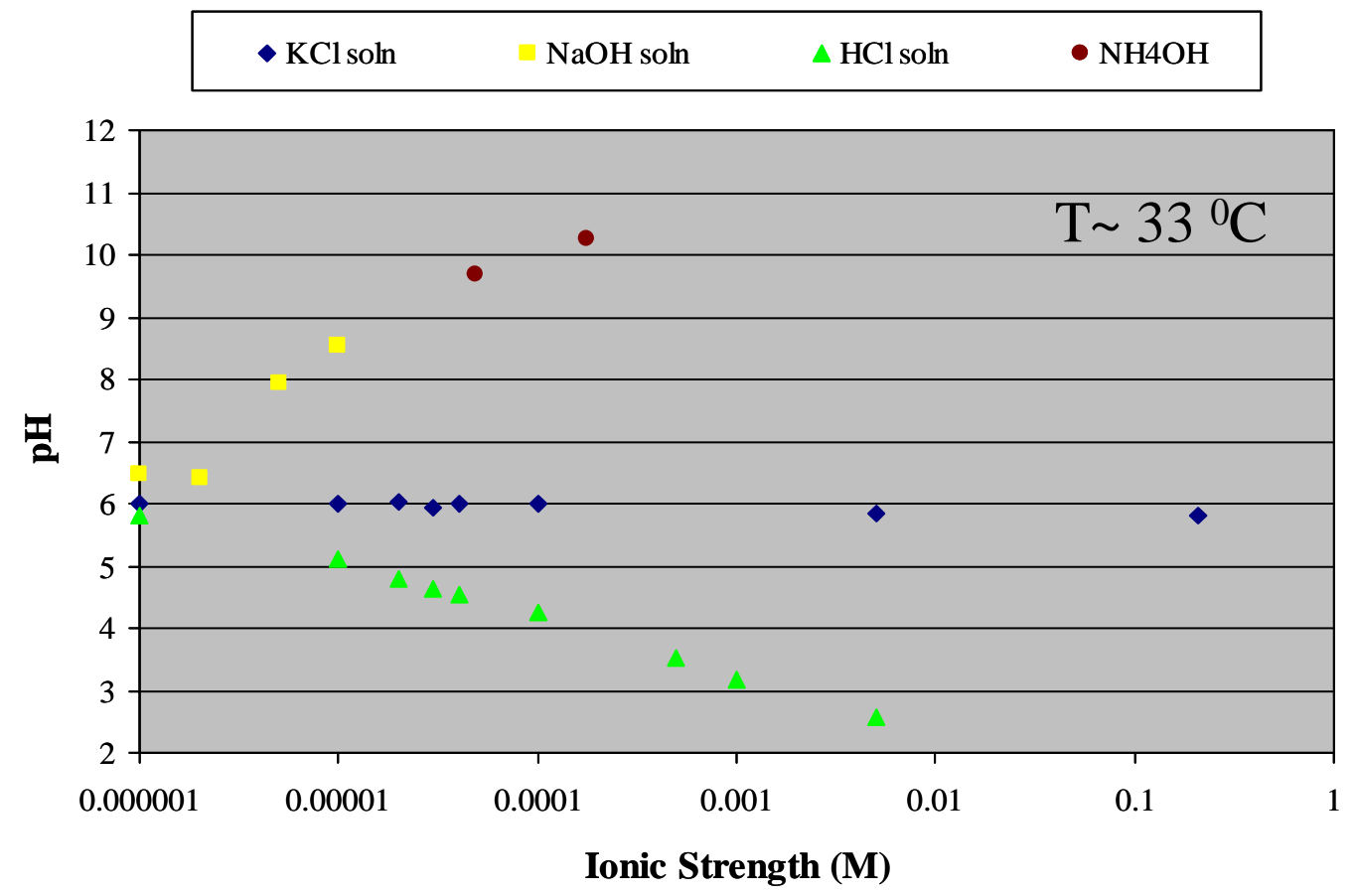

Figure 4.26: Solution $\mathrm{pH}$ of $\mathrm{HCl}, \mathrm{NaOH}, \mathrm{NH}_{4} \mathrm{OH}$ and $\mathrm{KCl}$ at $33{ }^{0} \mathrm{C}$ as a function of ionic strengths 
The cleaning results, shown in figure 4.27, indicate that the removal of aminated silica particles was much superior in alkaline $\mathrm{NaOH}$ solutions compared to near neutral $\mathrm{KCl}$ solutions at comparable solution ionic strengths. In $5 \times 10^{-5} \mathrm{M}$ of $\mathrm{NaOH}$ solution with $\mathrm{pH}$ of 8.5 , approximately $90 \%$ of silica particles were removed while in the $\mathrm{KCl}$ solution of an equivalent concentration and $\mathrm{pH}$ of 6.0 , the removal efficiency was only $45 \%$. Similarly, the particle removal efficiencies were better in $\mathrm{NH}_{4} \mathrm{OH}$ solutions compared to $\mathrm{KCl}$ solutions. On the other hand, when cleaning of wafers was performed in acidic conditions using $\mathrm{HCl}$, no significant difference in PREs was observed for $\mathrm{HCl}$ and $\mathrm{KCl}$ solutions. At solution ionic strength of $3 \times 10^{-5} \mathrm{M}$, the particle removal efficiencies were $50 \%$ in both $\mathrm{HCl}(\mathrm{pH}=4.6)$ and $\mathrm{KCl}(\mathrm{pH}=6.0)$ solutions. With an increase of ionic strength to $10^{-3} \mathrm{M}$, the PREs increased to $80 \%$ and $70 \%$ in $\mathrm{HCl}(\mathrm{pH}=3.2)$ and $\mathrm{KCl}$ solutions $(\mathrm{pH}=6.0)$ respectively.

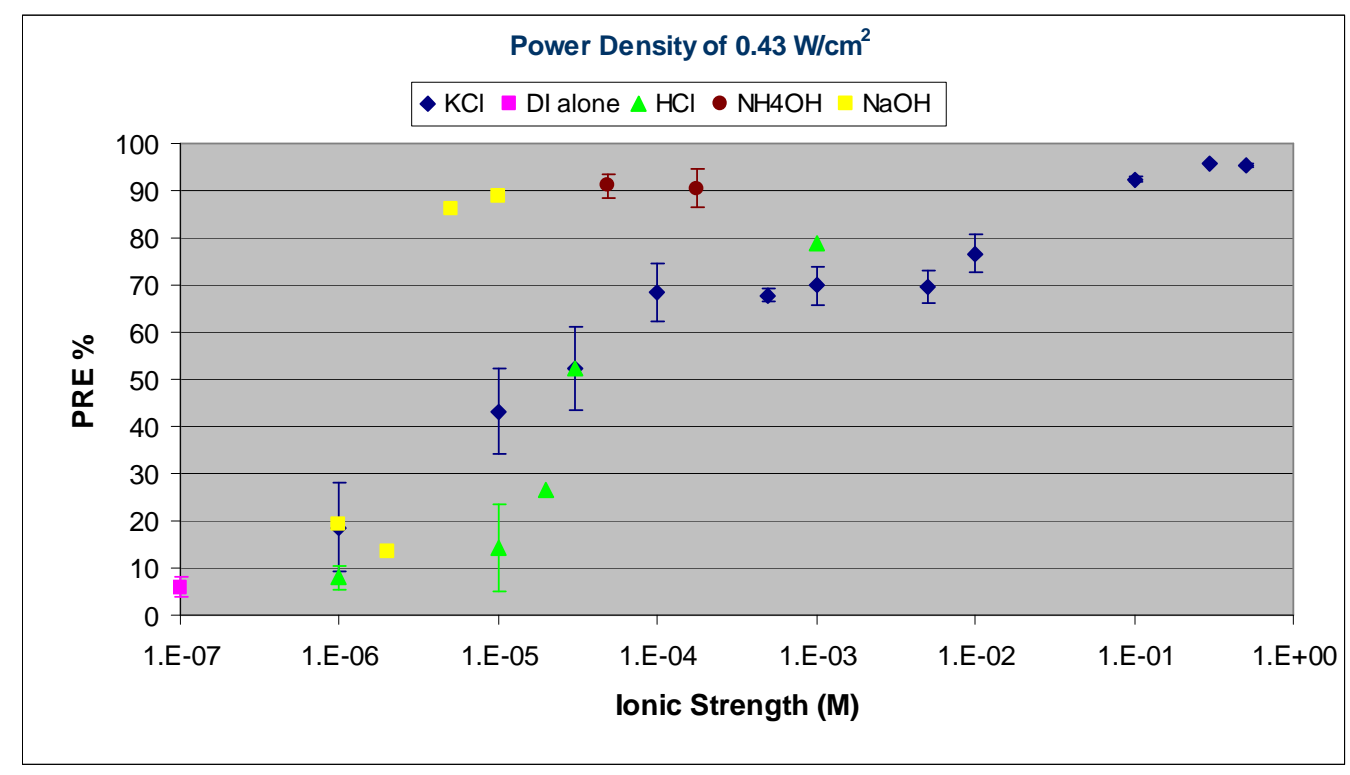

Figure 4.27: Removal of aminated silica particles from wafer surfaces in solutions of different $\mathrm{pH}$ at a megasonic power density of $0.43 \mathrm{~W} / \mathrm{cm}^{2}$ and a temperature of $30{ }^{\circ} \mathrm{C}$ 
The enhanced removal of aminated silica particles in alkaline $\mathrm{pH}$ is due to two effects: 1) particle surface charge reversal in alkaline $\mathrm{pH}$ and 2) etching of silicon dioxide film from the wafer. During deposition of aminated silica particles on an oxide wafer surface at $\mathrm{pH}$ of 6.0 , the surface charge on the particles is positive as evidenced by figure 4.1. Since the oxide surface is expected to be negative at this $\mathrm{pH}$, the electrostatic force between the particles and the surface is attractive causing the particles to adhere strongly on the wafer surface. During cleaning of wafers in an alkaline $\mathrm{pH}$ solution, the surface charge on the aminated silica particles gets reversed, resulting in a repulsive electrostatic force between the particles and the wafer surface. If the particles can be slightly moved from their equilibrium position by the sonic field, the electrostatic repulsive force is likely to remove the particles and also prevent their re-deposition.

At alkaline $\mathrm{pH}$ values, due to etching of the $\mathrm{SiO}_{2}$ film, the separation distance between the particles and the wafer may also be expected to increase which will reduce the van der Waals force of adhesion. The lower adhesion force can lead to higher removal of particles from wafers. In order to further explore the role of etching in removal of particles, the cleaning of wafers was carried out in solutions of different $\mathrm{pH}$ at $30{ }^{0} \mathrm{C}$ but in the absence of a megasonic field. Since the amount of $\mathrm{SiO}_{2}$ etched increases with time, the removal of particles was also studied as a function of cleaning time. Although cleaning experiments were performed both in acidic and alkaline conditions, no etching of $\mathrm{SiO}_{2}$ can be expected in low (acidic) $\mathrm{pH}$ solutions. The alkaline $\mathrm{pH}$ values of $11.5-$ 12.0 were achieved using $\mathrm{NH}_{4} \mathrm{OH}$ and those in acidic conditions between 2.4 and 2.6 
were adjusted using $\mathrm{HCl}$. The cleaning results, shown in figure 4.28, indicate that removal of aminated silica particles was insignificant, at any solution $\mathrm{pH}$ and cleaning time, in the absence of a megasonic field. Even at a high value of alkaline $\mathrm{pH}$ of 11.95 and cleaning time of 10 min, PRE was lower than $10 \%$. At this $\mathrm{pH}$ value, silicon etch rates of $\sim 5 \AA / m i n$ exist as estimated from [4.8]. This suggests that etching of oxide and reversal of particle surface charge in the absence of megasonics is unable to remove particles from the wafer surface. However, the two effects when combined in the presence of a megasonic field provide much better removal efficiencies compared to that in $\mathrm{KCl}$ solutions.

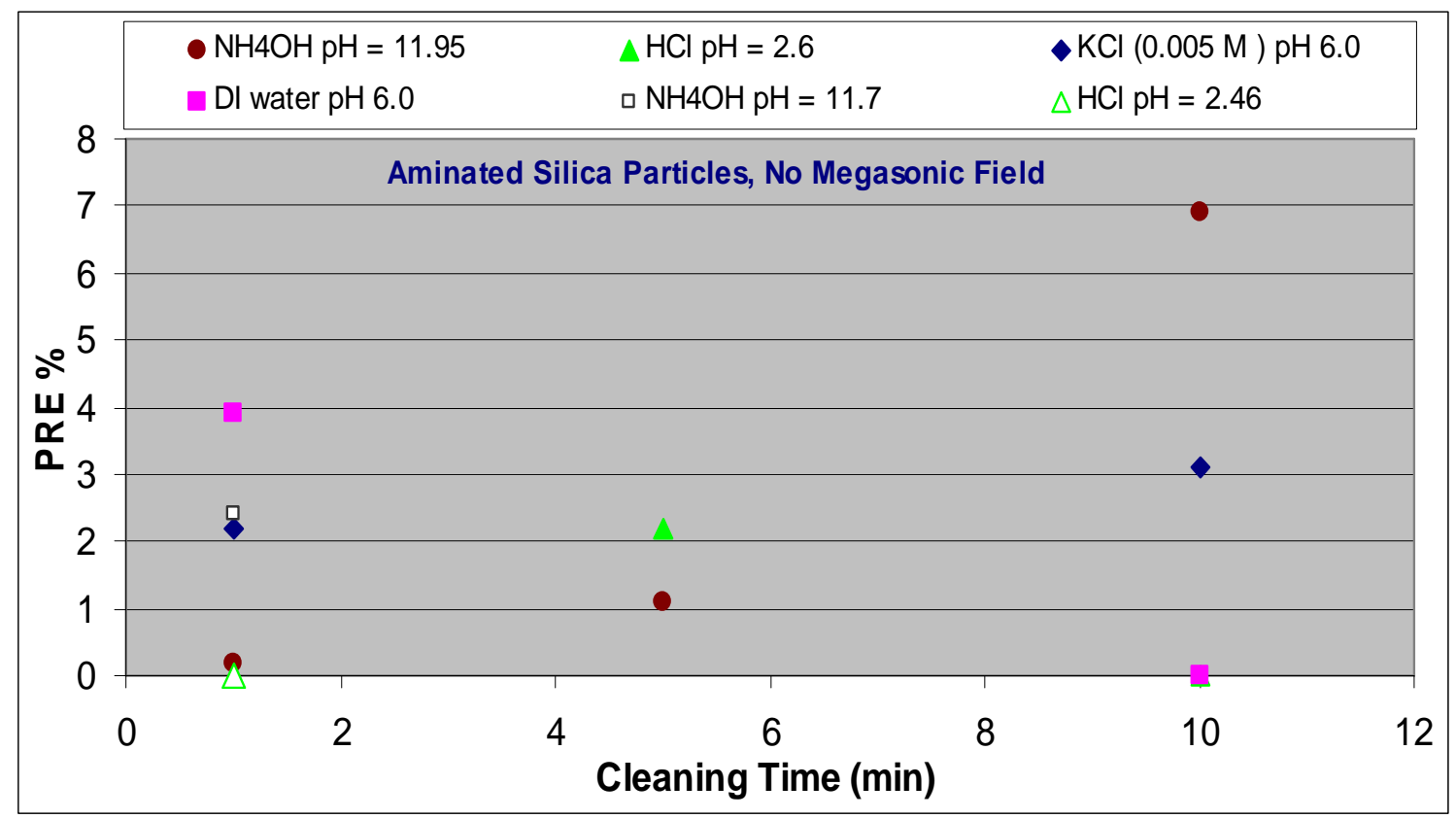

Figure 4.28: Removal of aminated silica particles in solutions of different $\mathrm{pH}$ in the absence of megasonic field at $30{ }^{\circ} \mathrm{C}$ 


\subsubsection{Effect of Cleaning Time on Removal of Aminated Silica Particles}

In order to study the effect of cleaning time on the removal of aminated silica particles, cleaning of wafers was performed in a $10^{-3} \mathrm{M} \mathrm{KCl}$ solution at $0.103 \mathrm{~W} / \mathrm{cm}^{2}$ for three different cleaning times of one, two and five minutes. The results, shown in figure 4.29, suggest that PRE increases significantly with cleaning time. The PRE increases approximately from $30 \%$ to $95 \%$ with an increase in cleaning time from 1 min to 5 min.

It must be noted that although longer cleaning times provide better removal of particles, the process also requires higher energy. Additionally, higher cleaning time means greater possibility of damage to features in the case of patterned wafers.

The higher PRE observed with cleaning time suggests that the cleaning mechanism for particle removal may not be instantaneous. Cavitation is a non-uniform process and therefore removal of particles due to cavitation can be expected to increase with time. Electro-acoustic effects that are uniform all over the wafer surface are likely to instantaneously dislodge the particles from the wafer surface. However, since the transport of particles from the viscous boundary layer to the bulk of the solution is known to increase with cavitation or micro-streaming, the removal of particles due to electroacoustic effects can also depend on time. 


\subsection{KCl solution, Power Density $=0.103 \mathrm{~W} / \mathrm{cm}^{2}$}

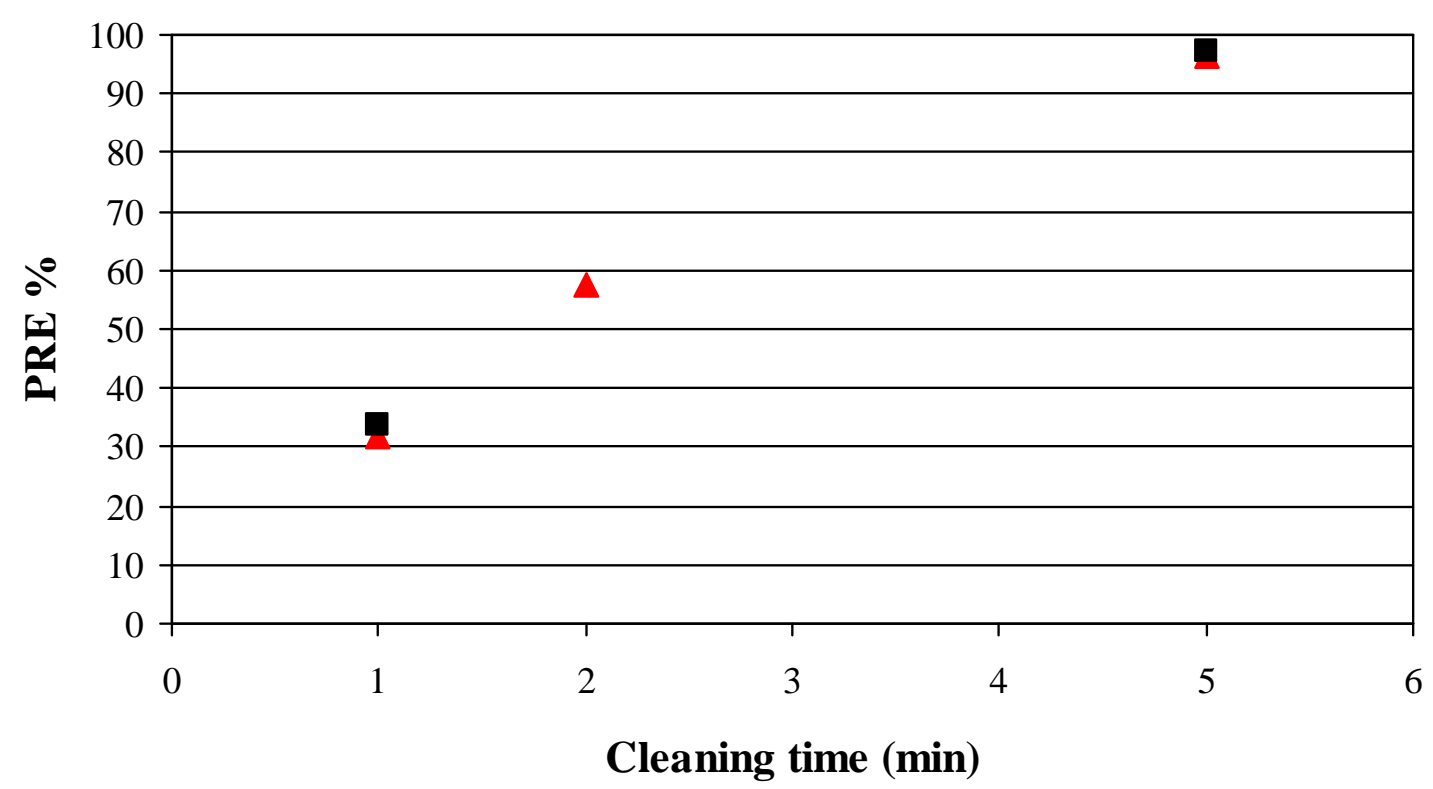

Figure 4.29: Removal of aminated silica particles in $10^{-3} \mathrm{M} \mathrm{KCl}$ solution at $0.103 \mathrm{~W} / \mathrm{cm}^{2}$ as a function of cleaning time

\subsubsection{Use of Other Chloride Based Electrolytes on Removal of Aminated Silica Particles}

The wafer cleaning experiments described in earlier sections used $\mathrm{KCl}$ as an electrolyte for the removal of aminated silica particles. In order to compare the performance of $\mathrm{KCl}$ with other electrolytes, tests were performed using sodium chloride $(\mathrm{NaCl})$, lithium chloride $(\mathrm{LiCl})$ and cesium chloride $(\mathrm{CsCl})$ at four different megasonic power densities of $0.43 \mathrm{~W} / \mathrm{cm}^{2}, 0.129 \mathrm{~W} / \mathrm{cm}^{2}, 0.103 \mathrm{~W} / \mathrm{cm}^{2}$ and $0.077 \mathrm{~W} / \mathrm{cm}^{2}$ and a solution temperature of $30{ }^{0} \mathrm{C}$. The particle removal efficiencies as a function of solution ionic strengths for different electrolytes and megasonic power densities are illustrated in 
figures $4.30-4.33$. At $0.129 \mathrm{~W} / \mathrm{cm}^{2}$ of megasonic power density in $10^{-4} \mathrm{M}$ of solution ionic strength, the particle removal efficiency was highest in $\mathrm{NaCl}$ solution followed by that in $\mathrm{CsCl}, \mathrm{KCl}$ and $\mathrm{LiCl}$ in decreasing order. On the other hand, at $0.103 \mathrm{~W} / \mathrm{cm}^{2}$ of power density and $10^{-4} \mathrm{M}$ of ionic strength, $\mathrm{CsCl}$ provided better particle removal than $\mathrm{KCl}$ and $\mathrm{LiCl}$ with lowest particle removal in the $\mathrm{NaCl}$ solution. Similarly, at other conditions, the order of performances of electrolytes varied depending on megasonic power densities and solution ionic strengths.

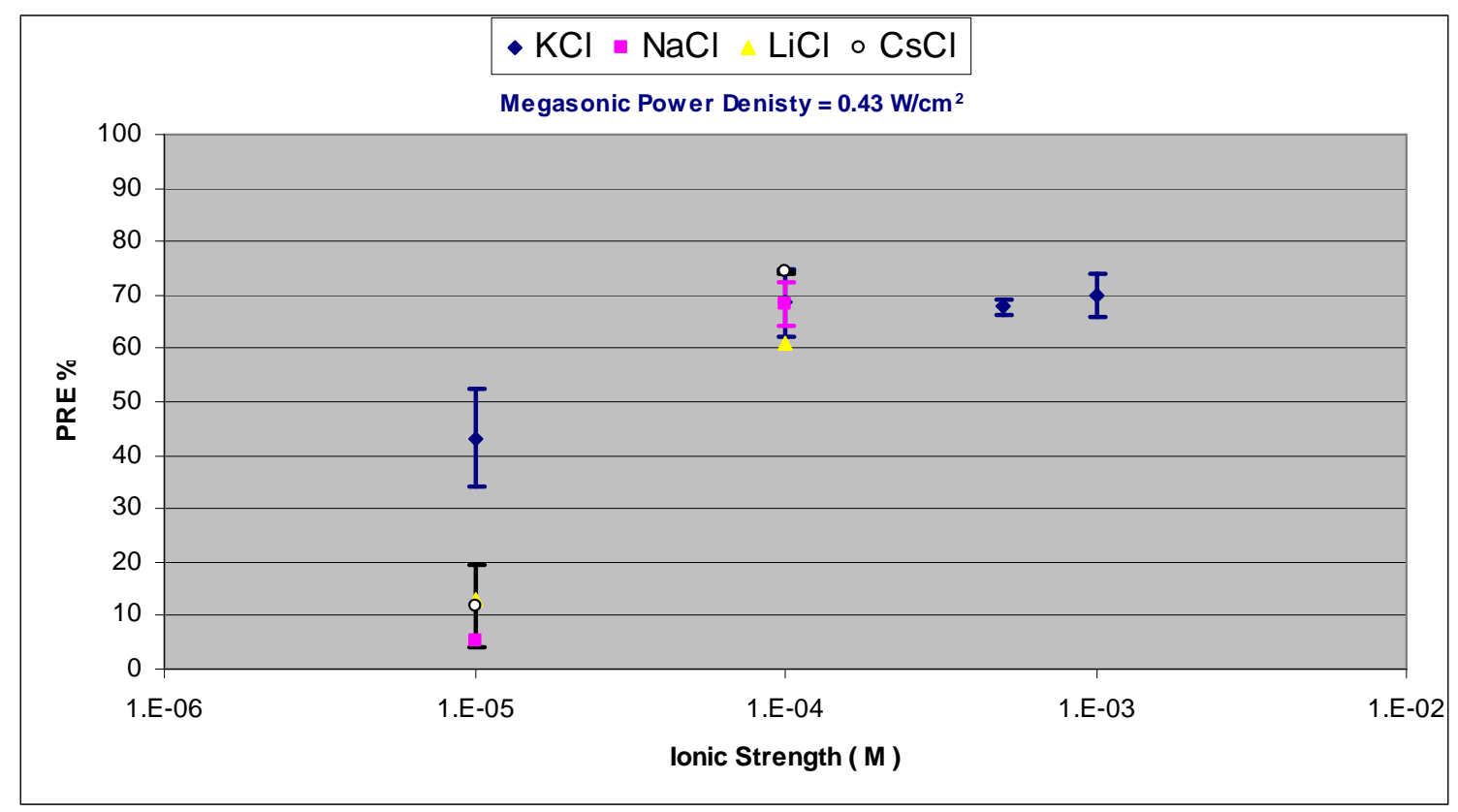

Figure 4.30: Removal of aminated silica particles in solutions of different electrolytes at $0.43 \mathrm{~W} / \mathrm{cm}^{2}$ (Solution Conditions: $\mathrm{pH}=6.0$, Temperature $=30{ }^{0} \mathrm{C}$ ) 


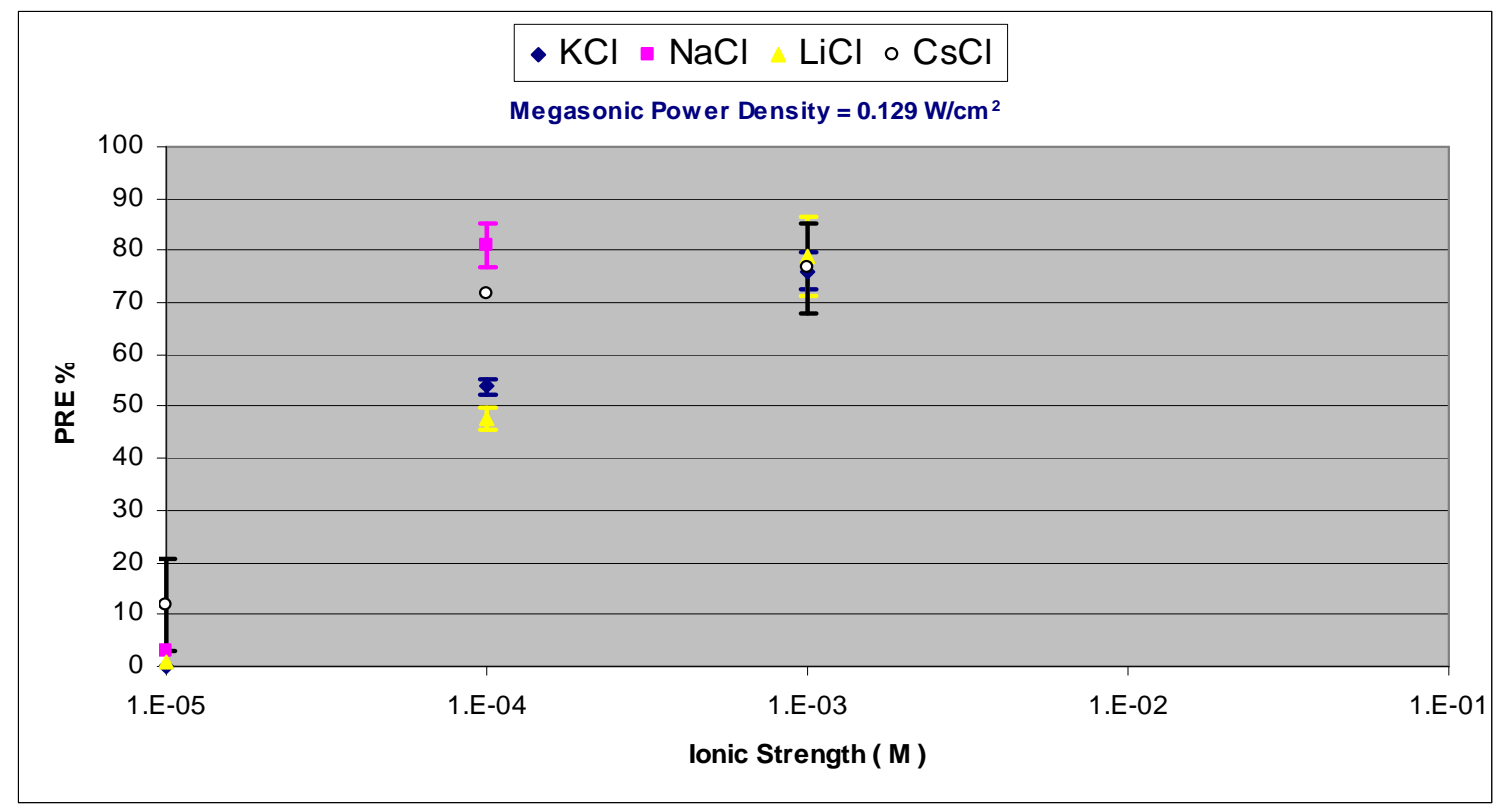

Figure 4.31: Removal of aminated silica particles in solutions of different electrolytes at $0.129 \mathrm{~W} / \mathrm{cm}^{2}$ (Solution Conditions: $\mathrm{pH}=6.0$, Temperature $=30{ }^{\circ} \mathrm{C}$ )

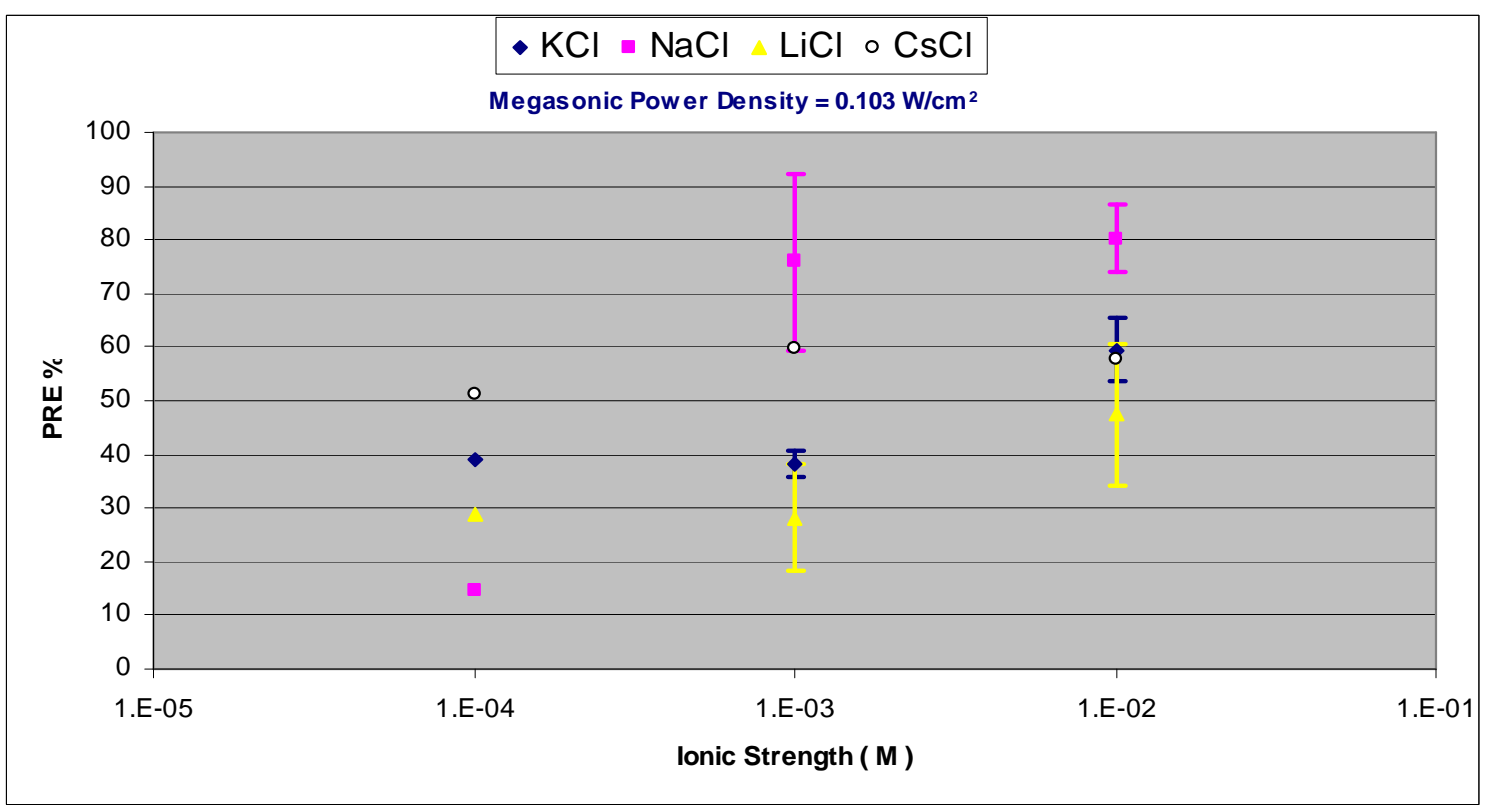

Figure 4.32: Removal of aminated silica particles in solutions of different electrolytes at $0.103 \mathrm{~W} / \mathrm{cm}^{2}$ (Solution Conditions: $\mathrm{pH}=6.0$, Temperature $=30{ }^{\circ} \mathrm{C}$ ) 


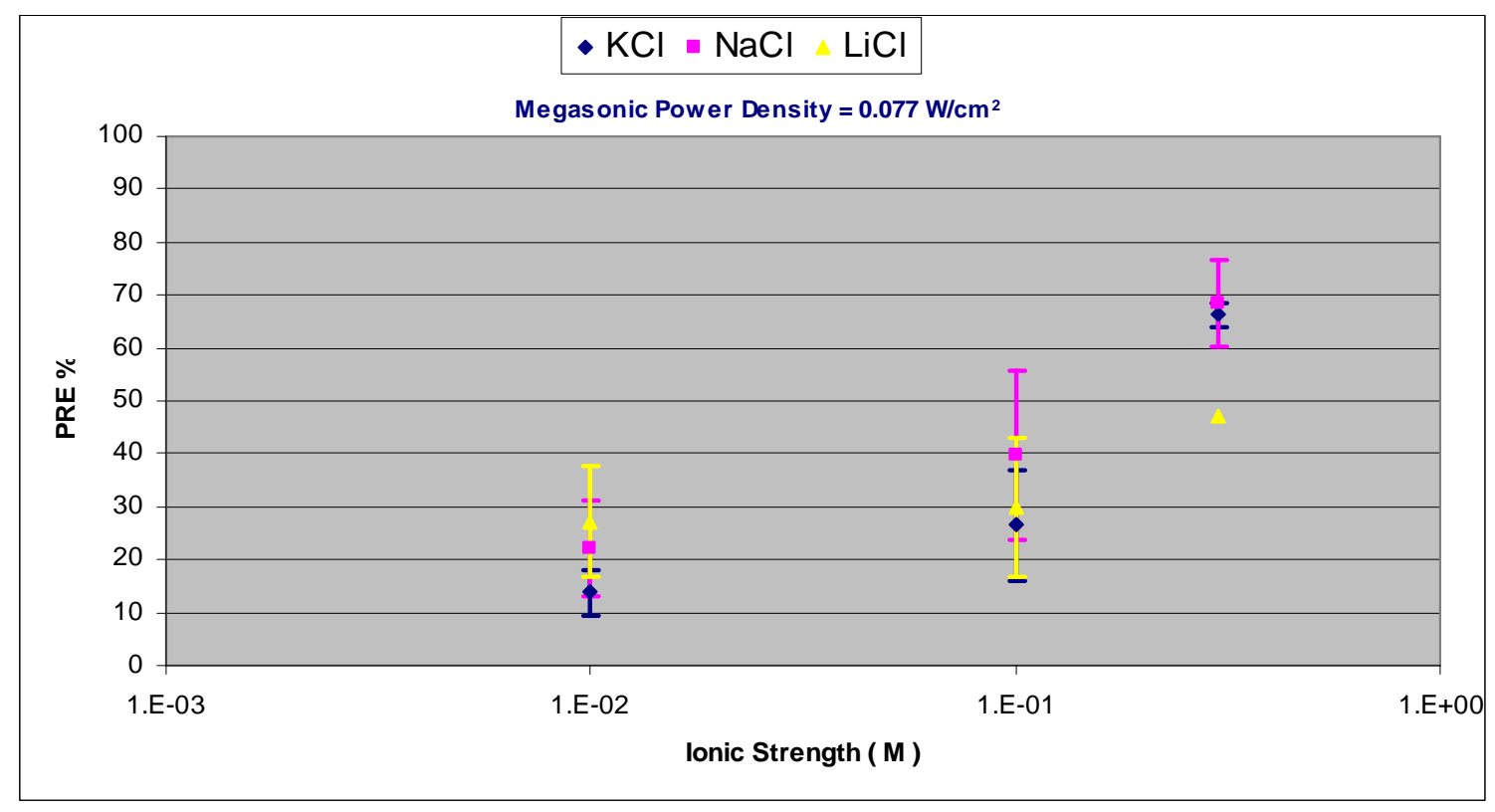

Figure 4.33: Removal of aminated silica particles in solutions of different electrolytes at $0.077 \mathrm{~W} / \mathrm{cm}^{2}$ (Solution Conditions: $\mathrm{pH}=6.0$, Temperature $=30^{\circ} \mathrm{C}$ )

In order to obtain a statistically valid comparison of performance between different electrolytes, Wilcoxon Matched-Pairs Signed Rank test (WMSRS) was employed. WMSRS is a non-parametric inferential statistical test that evaluates rank-order data. The details of the test may be obtained from Appendix A.3. Since the sample $(\mathrm{NaCl}, \mathrm{KCl}$, $\mathrm{CsCl}$ or $\mathrm{LiCl}$ ) scores (PRE) depend strongly on experimental conditions (power density and ionic strength), common or matched conditions were used for sample comparison. The number of sample data points used in the analysis for the two samples were equal. First, a difference in scores was obtained between the samples. The score difference was ranked with respect to their absolute values. Then the sign of each difference score was placed in front of its rank. The sum of ranks that had positive and the sum of ranks that had negative signs were estimated. 
Table 4.2 shows sample calculations for a comparison between $\mathrm{NaCl}$ and $\mathrm{KCl}$ following the Wilcoxon test procedure. The outcome was then evaluated in accordance with the ranking protocol. The electrolytes were ranked based on their overall performance. According to the analysis, the probability of the outcome due to chance was estimated to be less than $5 \%$. In table 4.3 , the results indicate that the overall performance of $\mathrm{NaCl}$ and $\mathrm{CsCl}$ was superior to that of $\mathrm{KCl}$ and $\mathrm{LiCl}$. The chloride solutions differ in their cation. The hydrated masses of cations, which may affect the dipole moment, follow the order $\mathrm{Cs}^{+}>\mathrm{Na}^{+}>\mathrm{K}^{+}>\mathrm{Li}^{+}$. The removal data does not follow this trend very well. With limited data available at the present time it is difficult to explain the results.

The Debye potential in these electrolyte solutions was also computed based on the hydrated masses of the ions as shown in figure 4.34. Although the potential increases by a few times in $\mathrm{CsCl}$ solution compared to that in $\mathrm{LiCl}$ or $\mathrm{KCl}$ solution, the electroacoustic force due to this potential is much lower than the van der Waals adhesion force. Thus, the reasons for different performances observed in electrolyte solutions are unknown at this time and further investigation is required. 
Table 4.2: Sample calculations for comparison between $\mathrm{NaCl}$ and $\mathrm{KCl}$ following the Wilcoxon test procedure

\begin{tabular}{|c|c|c|c|c|c|}
\hline $\begin{array}{l}\text { Experimental } \\
\text { Conditions }\end{array}$ & $\begin{array}{l}\text { Sample A }(\mathrm{NaCl}) \\
\text { scores }(\mathrm{X} 1)\end{array}$ & $\begin{array}{l}\text { Sample B (KCl) } \\
\text { scores }(\mathrm{X} 2)\end{array}$ & $\begin{array}{l}\mathbf{D}= \\
\mathbf{X} 1-\mathrm{X} 2\end{array}$ & $\begin{array}{l}\text { Rank } \\
\text { of }|D|\end{array}$ & $\begin{array}{l}\text { Signed } \\
\text { Rank of }|D|\end{array}$ \\
\hline $0.0001 \mathrm{M}, 0.103 \mathrm{~W} / \mathrm{cm}^{2}$ & 15 & 39 & -24 & 10.5 & -10.5 \\
\hline $0.001 \mathrm{M}, 0.103 \mathrm{~W} / \mathrm{cm}^{2}$ & 64 & 40 & 24 & 10.5 & 10.5 \\
\hline $0.001 \mathrm{M}, 0.103 \mathrm{~W} / \mathrm{cm}^{2}$ & 88 & 37 & 51 & 15 & 15 \\
\hline $0.01 \mathrm{M}, 0.103 \mathrm{~W} / \mathrm{cm}^{2}$ & 75 & 64 & 11 & 7 & 7 \\
\hline $0.01 \mathrm{M}, 0.103 \mathrm{~W} / \mathrm{cm}^{2}$ & 86 & 55 & 30 & 14 & 14 \\
\hline $0.0001 \mathrm{M}, 0.129 \mathrm{~W} / \mathrm{cm}^{2}$ & 84 & 55 & 29 & 13 & 13 \\
\hline $0.0001 \mathrm{M}, 0.129 \mathrm{~W} / \mathrm{cm}^{2}$ & 78 & 53 & 25 & 12 & 12 \\
\hline $0.01 \mathrm{M}, 0.077 \mathrm{~W} / \mathrm{cm}^{2}$ & 16 & 17 & -1 & 1.5 & -1.5 \\
\hline $0.01 \mathrm{M}, 0.077 \mathrm{~W} / \mathrm{cm}^{2}$ & 29 & 11 & 18 & 9 & 9 \\
\hline $0.1 \mathrm{M}, 0.077 \mathrm{~W} / \mathrm{cm}^{2}$ & 28 & 19 & 9 & 6 & 6 \\
\hline $0.1 \mathrm{M}, 0.077 \mathrm{~W} / \mathrm{cm}^{2}$ & 51 & 34 & 17 & 8 & 8 \\
\hline $0.3 \mathrm{M}, 0.077 \mathrm{~W} / \mathrm{cm}^{2}$ & 74 & 68 & 7 & 5 & 5 \\
\hline $0.3 \mathrm{M}, 0.077 \mathrm{~W} / \mathrm{cm}^{2}$ & 63 & 65 & -2 & 3.5 & -3.5 \\
\hline $0.0001 \mathrm{M}, 0.43 \mathrm{~W} / \mathrm{cm}^{2}$ & 66 & 64 & 1 & 1.5 & 1.5 \\
\hline $0.0001 \mathrm{M}, 0.43 \mathrm{~W} / \mathrm{cm}^{2}$ & 71 & 73 & -2 & 3.5 & -3.5 \\
\hline
\end{tabular}

Table 4.3: Summary of performance ranking of electrolytes obtained after statistical analysis using Wilcoxon Matched-Pairs Signed Rank test

\begin{tabular}{|c|c|}
\hline Electrolyte & $\begin{array}{c}\text { Rank } \\
\text { (lower value of rank refers to } \\
\text { better performance) }\end{array}$ \\
\hline $\begin{array}{c}\text { \% likelihood that the outcome is due to } \\
\text { chance } \leq 5 \%\end{array}$ \\
\hline $\mathrm{NaCl}$ & 1 \\
\hline $\mathrm{CsCl}$ & 1 \\
\hline $\mathrm{KCl}$ & 2 \\
\hline $\mathrm{LiCl}$ & 2 \\
\hline
\end{tabular}




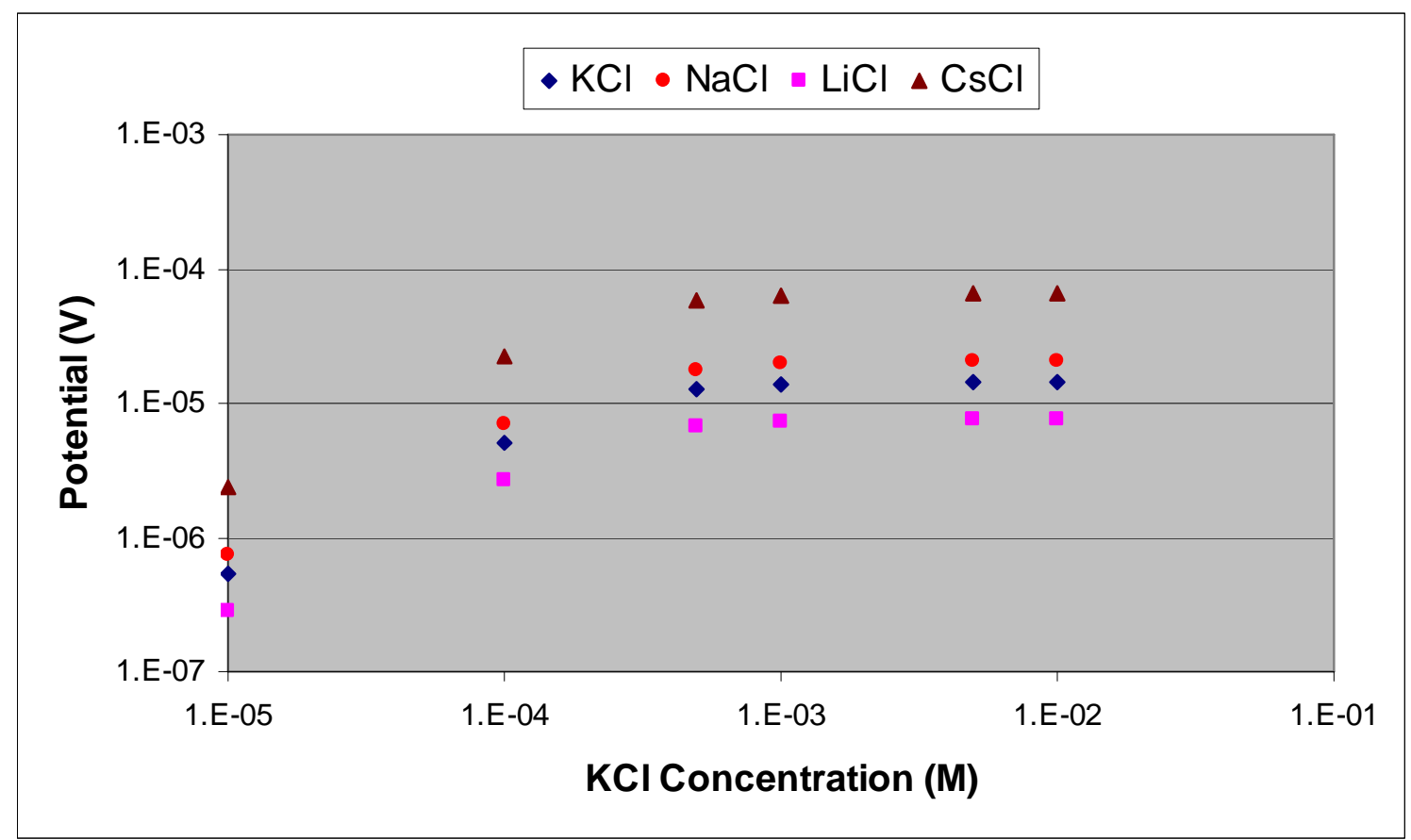

Figure 4.34: Debye potential in different electrolyte solutions at $0.43 \mathrm{~W} / \mathrm{cm}^{2}$ of megasonic power density at $30{ }^{\circ} \mathrm{C}$ 


\section{CHAPTER 5: CONCLUSIONS AND FUTURE WORK}

\subsection{Conclusions}

The following conclusions can be drawn from different studies in this dissertation:

1. Wafer cleaning experiments revealed that the removal of charged particles from surfaces in a megasonic field can be significantly improved by employing electrolyte solutions of varying ionic strengths. Studies performed using $\mathrm{KCl}$ as an electrolyte showed that the removal of silica particles from wafer surfaces was much higher in $\mathrm{KCl}$ solutions compared to that in DI water alone at all megasonic power densities. The critical electrolyte concentration, $\mathrm{C}_{\mathrm{c}}$, for removal of particles was found to increase with a decrease in transducer power density. Theoretical computations showed that removal forces due to CVP were much larger in magnitude than those due to IVP and were comparable to van der Waals adhesion forces.

2. Hydrophone studies revealed that the sound wave pressure amplitude can be increased in electrolyte solutions of ionic strengths greater than $0.01 \mathrm{M}$. Fast Fourier Transform of pressure amplitude data in $\mathrm{KCl}$ solutions showed that higher pressure amplitude results in wider distribution of oscillating stable bubbles. Sonoluminescence experiments in $\mathrm{KCl}$ solutions of concentrations of 
0.6 $\mathrm{M}$ and $1.1 \mathrm{M}$ demonstrated that $\mathrm{KCl}$ solutions exhibit more intense cavitation than DI water. These studies, in combination, suggested that both stable and transient cavitation were higher in $\mathrm{KCl}$ solutions at concentrations greater than $0.01 \mathrm{M}$ compared to that in DI water, and therefore could provide additional force for removal of particles from wafers.

3. The effect of solution variables such as temperature and $\mathrm{pH}$ on megasonic removal of aminated silica particles was found to be significant. The particle removal efficiency increased with temperature of $\mathrm{KCl}$ solution and power density.

4. Increasing the solution $\mathrm{pH}$ from near neutral to alkaline condition at equivalent solution ionic strength significantly improved the megasonic removal of aminated silica particles. The effect was attributed to the combined effect of particle surface charge reversal and oxide etching under alkaline $\mathrm{pH}$ conditions during megasonic cleaning. In the absence of a megasonic field, insignificant particle removal was observed for similar solution conditions.

5. The effect of cleaning time on removal of particles in $\mathrm{KCl}$ solutions showed that particle removal efficiency increased with time of cleaning. Since both electro-acoustic and cavitation effects can be time dependent, the removal of particles could be expected to be a function of time. 
6. Comparison of particle removal efficiencies in different electrolytes using Wilcoxon Matched-Pairs Signed Ranked test showed that the overall performance of sodium chloride and cesium chloride was superior to that of potassium chloride and lithium chloride.

\subsection{Future Work}

1. Study the effect of electrolytes containing anions other than $\mathrm{Cl}^{-}$on megasonic removal of aminated silica particles from wafer surfaces.

2. Carry out particle removal studies on patterned test structures. Relate particle removal to damage of structures.

3. Attempt to deconvolute the effect of cavitation from the electro-acoustic effect. This can be done using $\mathrm{CO}_{2}$ that is known to suppress cavitation. The ionic strength of the solution can be modulated by controlling the amount of $\mathrm{CO}_{2}$ dissolved.

4. Study the effect of electrolytes for particles with varying degree of adhesion to surface. Investigate the degree of adhesion by atomic force microscopy measurements. 


\section{APPENDIX A}

\section{A.1. Derivation of Streaming Equation}

Consider a homogenous isotropic fluid with pressure $p(x, y, z, t)$, density $\rho(x, y, z, t)$ and velocity $u(x, y, z, t)$ through which an acoustic wave is propagated. If $f$ is the net force per unit volume due to elasticity and both shear and bulk viscosity on a small volume element, then the dynamical equations are given as:

$$
\begin{aligned}
& f=\rho\left[\frac{\partial u}{\partial t}+(u \cdot \nabla) u\right] \\
& f=-\nabla p+\left[\mu^{\prime}+(4 / 3) \mu\right] \nabla \nabla \cdot u-\mu \nabla \mathrm{X} \nabla \mathrm{X} u
\end{aligned}
$$

where $\mu$ and $\mu^{\prime}$ are the shear and bulk viscosity for the fluid and are considered constant.

The continuity equation gives:

$$
\frac{\partial \rho}{\partial t}+\nabla \cdot \rho u=0
$$

Equations A.1, A.2 and A.3, when combined, give 


$$
\frac{\partial(\rho u)}{\partial t}+F=-\nabla p+\left(\mu^{\prime}+\frac{4 \mu}{3}\right) \nabla \nabla \cdot u-\mu \nabla \mathrm{X} \nabla \mathrm{X} u
$$

where

$$
F=\rho(u \cdot \nabla) u+u \nabla \cdot \rho u
$$

Nyborg made the following approximations for the excess pressure $p-p_{0}$ and the excess density $\rho-\rho_{0}$ and the fluid velocity $u$.

$$
p-p_{0}=p_{1}+p_{2}+\ldots
$$

$\rho-\rho_{0}=\rho_{1}+\rho_{2}+\ldots$

$$
u=u_{1}+u_{2}+\ldots
$$

Here $p_{1}, \rho_{1}$ and $u_{1}$ correspond to the pressure, density and velocity of the fluid particle that would vary sinusoidally in time with frequency $w$ and represent the sound field. The terms $p_{2}, \rho_{2}$ and $u_{2}$ represent the time independent second order approximations with $u_{2}$ being the streaming velocity that needs to be determined. 
Taking time average of equation A.4, one can obtain

$F^{*}=-\nabla p_{2}+\left[\mu^{\prime}+\frac{4 \mu}{3}\right] \nabla \nabla \cdot u_{2}-\mu \nabla \mathrm{X} \nabla \mathrm{X} u_{2}$

where

$F^{*}=\rho_{0}\left\langle\left(u_{1} \cdot \nabla\right) u_{1}\right\rangle_{t}+\rho_{0}\left\langle u_{1}\left(\nabla \cdot u_{1}\right)\right\rangle_{t}$

Taking $\left(\nabla \cdot u_{2}\right)=0$ (assuming $u_{2}$ to be solenoidal) we get

$\mu \nabla^{2} u_{2}=\nabla p_{2}+F^{*}$

As one can notice from these equations, time independent quantity $F^{*}$ is obtained by taking time average of quantity containing $u_{1}$ and derivatives of $u_{1}$. The Eckart streaming velocity is easily determined once $F^{*}$ is known. 


\section{A.1.1. Eckart Streaming Velocity in a Megasonic Cleaning Tank}

In a unidirectional flow, one can consider $u_{1}$ as a function of $x$ only, in which case

$u_{1 x}=U e^{-\alpha x} e^{i w\left(t-\frac{x}{c}\right)}$

where $\alpha=\frac{2 \pi^{2} f^{2}}{\rho_{0} c^{3}}\left[\frac{4 \mu}{3}+\mu^{\prime}\right], w=2 \pi f, f$ is the frequency in $\mathrm{Hz}, \alpha$ is the attenuation coefficient, $c$ is the speed of sound and $U$ is the maximum fluid velocity of the oscillating component $u_{1 x}$ calculated as $\left(\frac{2 I}{\rho_{0} c}\right)^{1 / 2}, I$ being the intensity of the sound source.

Solving for $F_{x}^{*}$ we get,

$F_{x}^{*}=-\rho_{0} \alpha U^{2} e^{-2 \alpha x}$

Since $\alpha x$ is much less than 1 , we can take the magnitude of ${F_{x}}_{x}$ as

$F_{x}^{*}=-\rho_{0} \alpha U^{2}$ 
Solving equation A.10, one can obtain the Eckart streaming velocity for free beam in an open channel (no pressure drop in the direction of flow of streaming velocity) as described in equations below [2.76]. The transducer and tank dimensions used for the calculations are shown in figure A.1.

$u_{2}=B[h-2 d] y \quad$ for $0 \leq y \leq d 0$

$u_{2}=B\left[h y-y^{2}-d^{2}\right]$ for $d \leq y \leq \frac{h}{2}$

$u_{2}=B\left\lfloor h(h-y)-(h-y)^{2}-d^{2}\right\rfloor$ for $\frac{h}{2} \leq y \leq\left(l+\frac{h}{2}\right)$

$u_{2}=B[h-2 d](h-y) \quad$ for $\left(l+\frac{h}{2}\right) \leq y \leq h$

where

$B=\frac{\rho_{0} \alpha U^{2}}{2 \mu}$ 


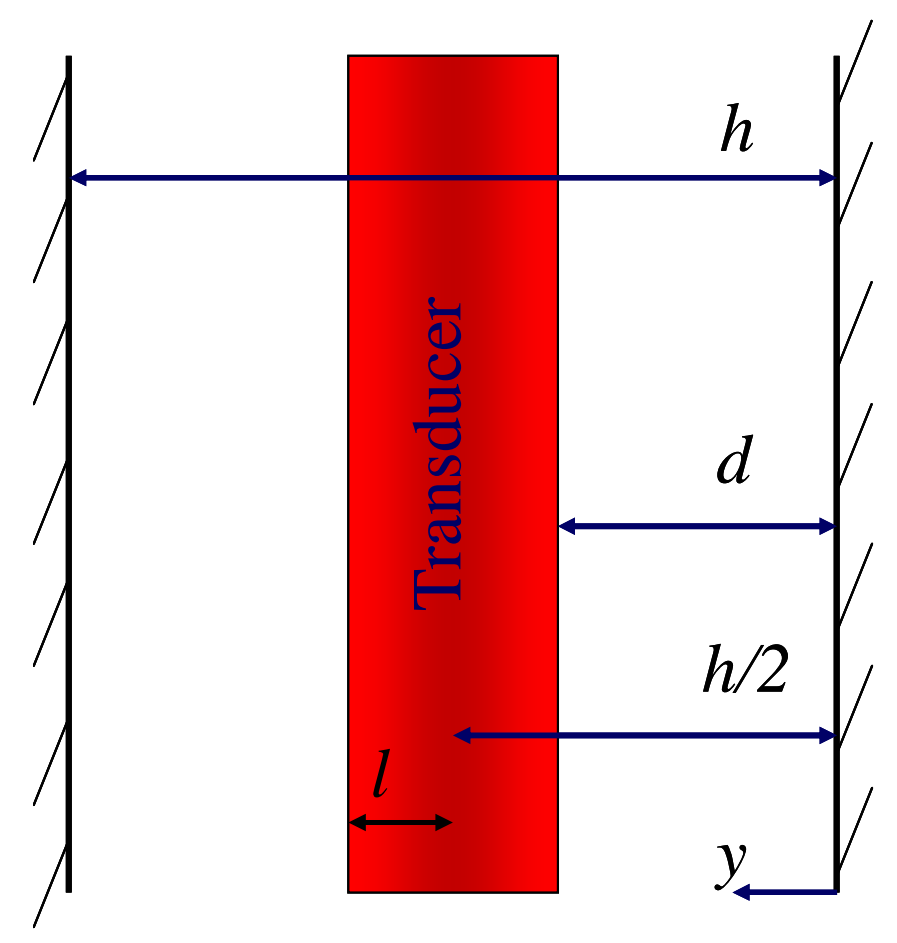

Figure A.1: Schematic for transducer in megasonic tank with different dimensions

Similarly, in a megasonic cleaning tank where the net flow of fluid is zero and the fluid essentially recirculates, a similar analysis, as above, gives the magnitude of the Eckart streaming velocity.

$$
\begin{aligned}
& u_{2}=B[h-2 d] y+(2 \mu)^{-1}\left[\mu B\left(2-3\left(\frac{2 d}{h}\right)^{2}+\left(\frac{2 d}{h}\right)^{3}\right)\right] y[y-h] \text { for } 0 \leq y \leq d \\
& u_{2}=B\left[h y-y^{2}-d^{2}\right]+(2 \mu)^{-1}\left[\mu B\left(2-3\left(\frac{2 d}{h}\right)^{2}+\left(\frac{2 d}{h}\right)^{3}\right)\right] y[y-h] \text { for } d \leq y \leq \frac{h}{2}
\end{aligned}
$$


Performing the calculations for water as the fluid in the megasonic tank and assuming the following values of different parameters to be used in the equations, the maximum streaming velocity at $\mathrm{y}=\mathrm{h} / 2$ is plotted as a function of transducer intensity for both cases of open and close ends in figures A.2 and A.3 respectively.

$f=0.925 \times 10^{6} \mathrm{~Hz}, \rho_{0}=998 \mathrm{Kg} / \mathrm{m}^{3}, \mu=0.001 \mathrm{~Pa}-\mathrm{sec}, \mu^{\prime}=3 \mu=0.003 \mathrm{~Pa}-\mathrm{sec}, c=1481$ $\mathrm{m} / \mathrm{sec}, d=11 \mathrm{~cm}=0.11 \mathrm{~m}, h=25 \mathrm{~cm}=0.25 \mathrm{~m}$

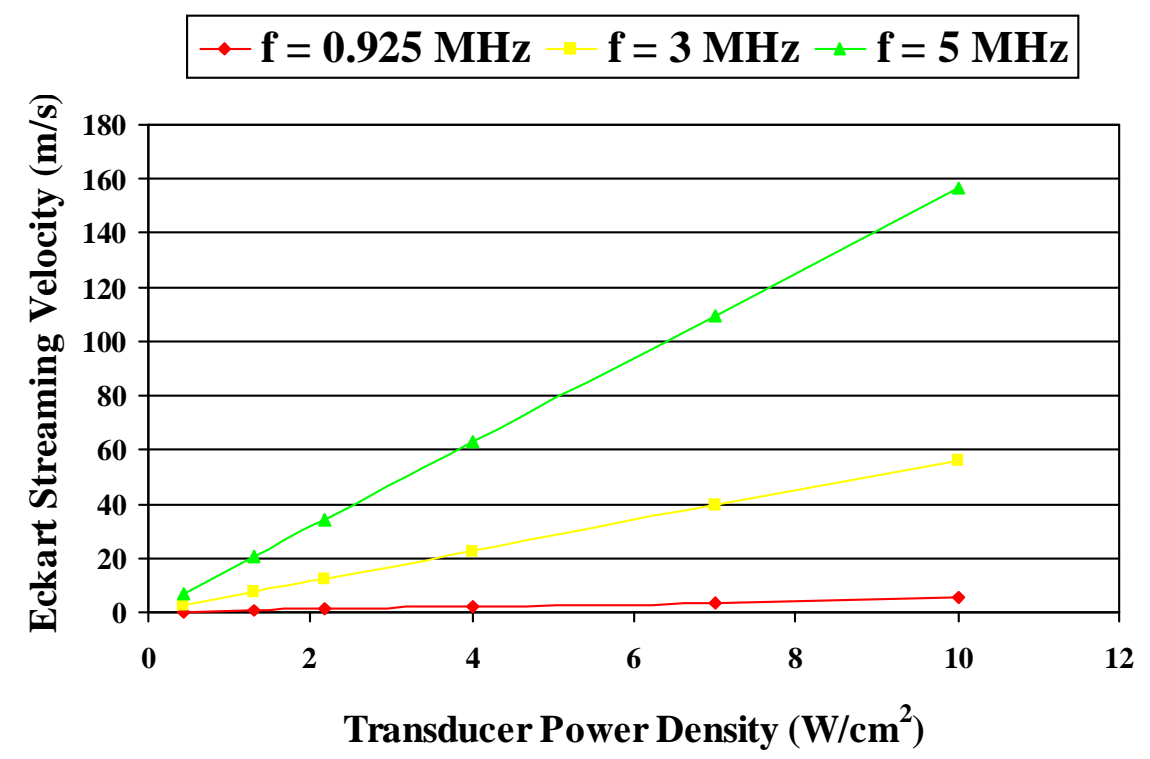

Figure A.2: Maximum Eckart streaming velocity at different transducer intensities and sound wave frequency for open channel 


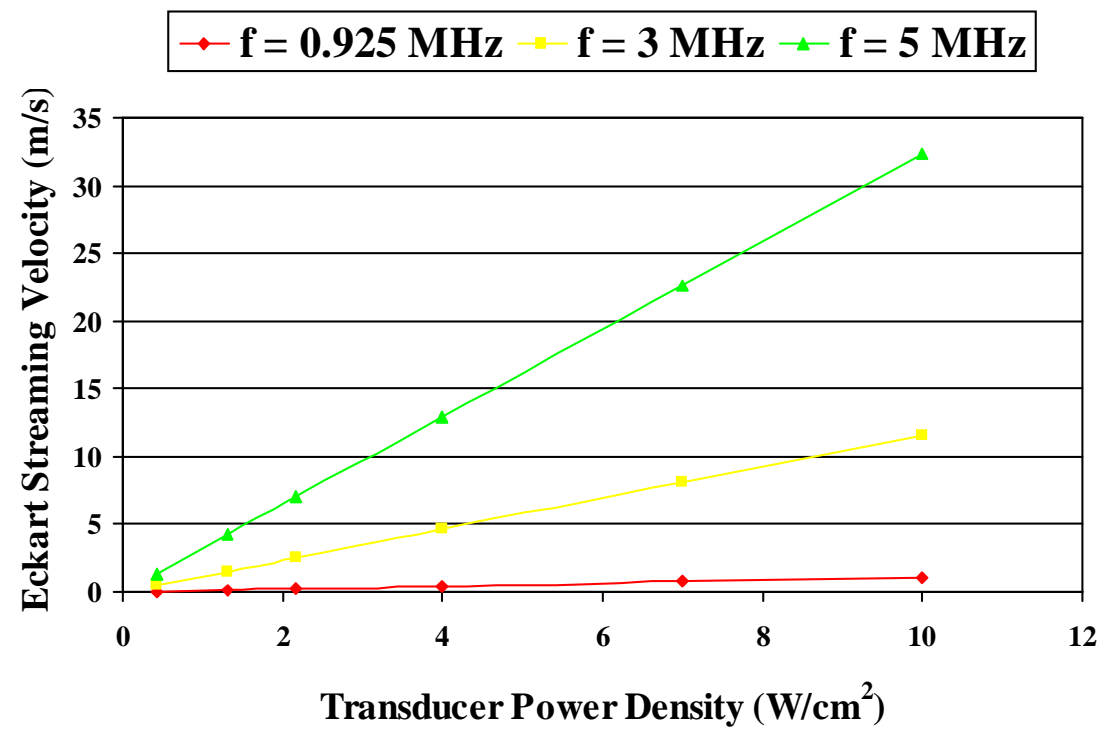

Figure A.3: Maximum Eckart streaming velocity at different transducer intensities and sound wave frequency for closed channel

\section{A.2. Wilcoxon Matched Pairs Signed Rank Test}

The Wilcoxon matched-pairs signed rank test is a non-parametric test that is used for statistical analysis of two dependent samples. This test assumes that the subjects have been randomly selected from a random population and the scores of the subjects are in interval/ratio format. The third assumption, that the distribution of difference in scores should be symmetric about the median of the population of difference scores, is not very restrictive. The following procedure is adopted when ranking the difference scores for the Wilcoxon matched-pairs signed-ranked test.

1) The absolute values of the difference in scores are ranked. 
2) Any difference in score that is zero is not ranked and is removed from the analysis.

3) The tied difference scores are averaged and the average score is given to each.

4) A rank of 1 is assigned to the difference score with lowest absolute magnitude. Similarly, a rank of $\mathrm{n}$ is given to the difference score with highest absolute magnitude, $\mathrm{n}$ being the number of non zero difference scores.

Once the difference scores are ranked, the sign of each difference score is placed in front of its rank. The sum of ranks that have positive $\left(\sum R+\right)$ and the sum of ranks that have negative signs $\left(\sum \mathrm{R}-\right)$ are estimated. If $\sum \mathrm{R}+$ and $\sum \mathrm{R}$ - are equal, then the medium of the difference score is zero and the two samples can be considered to be equivalent. If $\sum \mathrm{R}+$ is greater than $\sum \mathrm{R}$ - or vice versa, it indicates that there is a high likelihood that one of the samples represents a population with scores higher than the other. However, in order to know if the difference between the two samples is significant (not a result of chance), Critical one-tailed $\mathrm{T}$ values, shown in table A.1, are used. These $\mathrm{T}$ values depend on the number of ranked scores. First, the smaller of the two values, $\Sigma R+$ and $\sum \mathrm{R}-$, is designated as Wilcoxon $\mathrm{T}$ test statistic. If this computed value of $\mathrm{T}$ is equal to or less than the tabled critical $\mathrm{T}$ value at a prespecified level of significance, then the difference in population between the two samples is significant at that prespecified level of significance. For example, assume $\sum \mathrm{R}+$ and $\sum \mathrm{R}-$, obtained from sample A score minus sample B score, to be 40.5 and 4.5 respectively. Since $\sum R+$ is greater than $\sum R-$, the two populations of sample A and sample B are likely to be different. Further, since $\sum \mathrm{R}$ - is 
smaller than $\mathrm{T}_{0.05}$, overall sample A scores are larger than sample B scores, with less than $5 \%$ probability that the difference in scores between two samples is due to chance.

Table A.1: Tabled Critical Wilcoxon T values for $\mathrm{n}=9$ Signed Ranks

\begin{tabular}{|c|c|c|}
\hline & $\mathbf{T}_{0.05}$ & $\mathbf{T}_{0.01}$ \\
\hline One-tailed Values & 8 & 3 \\
\hline
\end{tabular}




\section{REFERENCES}

1.1.G. Moore, "Cramming More Components Onto Integrated Circuits," Electronics, Vol. $38,8(1965)$

1.2.W. Kern, The Evolution of Silicon Wafer Cleaning Technology, Journal of Electrochemical Society, vol 137, pp.1887 (1990)

1.3.ITRS, The International Technology Roadmap for Semiconductors (2007)

1.4.M. Tamaoki, K. Nishiki, A. Shimazaki, Y. Sasaki and S. Yanagi, The Effect of Airborne Contaminants in the Cleanroom for ULSI Manufacturing Process, IEEE/SEMI, Advanced Semiconductor Manufacturing Conference, pp. 322-326 (1995)

1.5.J. Plummer, M. Deal \& P. Griffin, Silicon VLSI Technology Fundamentals, Practice and Modeling, International Edition, Prentice Hall Inc. (2000)

1.6.W. Deen, Analysis of Transport Phenomena, Oxford University Press (1998)

1.7.H. Schlichting \& K. Gersten, Boundary Layer Theory, 8th Edition, Springer-Verlag (2001)

1.8.R. Donovan, Particle Control for Semiconductor Manufacturing, Marcel Dekker Inc. (1990)

1.9.W. Kern, Handbook of Semiconductor Wafer Cleaning Technology, Noyes Publications (1993)

1.10.W. Kern \& C. Deckert, Thin Film Processes, Academic Press, NY, Chapter V-1, pp. 401-496 (1978)

1.11.W. Kern \& G. Schnable, The Chemistry of Semiconductor Industry, Chapter 11, pp. 223-276, Chapman and Hall (1987)

1.12.W. Sievert, Buffered hydrofluoric acid: Wet processing is still going strong, Article ID 11062, SpecChemonline, April (2006) http://www.specchemonline.com/shownews.asp?secid=7\&nav=1\&newstype=\&key $=\&$ page $=\&$ newsid $=11062$

1.13.M. Niwano, J. Kageyama, K. Kinashi, \& N. Miyamoto, Infrared Spectroscopic Study of Initial Stages of Ultraviolet Ozone Oxidation of Si (100) and (111) 
Surfaces, The Journal Of Vacuum, Science Technology, vol A12, 2, pp. 465-470 (1994)

1.14.I. Boyd, R. Gwilliam and A. Kazor, Growth Rate Enhancement using Ozone during Rapid Thermal Oxidation of Silicon, Applied Physics Letters, vol 65, 4, pp. 412-414 (1994)

1.15.J. Vig, in Semiconductor Cleaning Technology, The Electrochemical Society Proceedings Series, 90-9, 105 (1989)

1.16.A. Licciardello, O. Puglisi and S. Pignataro, Effect of Organic Contaminants on the Oxidation Kinetics of Silicon at Room temperature, Applied Physics Letters, vol 48, pp. 41-43 (1986)

1.17.F. Smedt, S. Gendt, M. Heyns and C. Vinckier, The Application of Ozone in Semiconductor Cleaning Processes: The solubility Issue, Journal of The Electrochemical Society, 148, 9, G487-G493 (2001)

1.18.H. Abe, H. Iwamoto, T. Toshima, T. Iino, and G. Gale, Novel Photoresist Stripping Using Ozone/Vaporized Water Mixture, IEEE Transactions on Semiconductor Manufacturing, 16, 3 August (2003)

1.19.H. Tomita, M. Sato, S. Nadahara, and T. Saitoh, Photo Resist Stripping Using Novel Sulfuric/Ozone Process, IEEE (2001)

1.20.S. Raghavan \& M. Keswani, Thermal and Chemical Characteristics of SPM Systems For Resist Stripping Applications, SEMICON Korea 2008

1.21.A. Ermakov, I. Larin, Y. Kozlov and A. Purmal, The Thermodynamic Characteristics of Hydrogen Peroxide in H2SO4-H2O Solutions, Russian Journal of Physical Chemistry, 80, 12, pp 1895-1901 (2006)

1.22.W. Kern and D.A. Puotinen, Cleaning Solution Based on Hydrogen Peroxide for Use in Semiconductor Industry, RCA Rev., vol 31, pp. 187-206 (1970)

1.23.G. Olah and A. Molnar, Hydrocarbon Chemistry, Second Edition, WileyInterscience (2003)

1.24.X. Zhang, Electrochemistry of Silicon and Its Oxide, Springer (2001)

1.25.Prof. S. Raghavan, MSE 546: Semiconductor Processing Class Notes, The University of Arizona (2007) - Online Webpage, http://www.mse.arizona.edu/classes/mse446/ 
1.26.C. James, Chemistry of waste minimization, Springer (1995)

1.27.J. Ruzyllo, Wet Cleaning technology-Implementation, Semiconductor note 17, April 19 (2006)

1.28.J D. Burkman, Optimizing the Cleaning Procedure for Silicon Wafers Prior to High Temperature Operations, Semiconductor International, vol 4, 7, pp. 103-114 (1981)

1.29.R. Chein and W. Liao, Modeling of Particle Removal using Non-contact Brush Scrubbing in Post-CMP Cleaning Processes, The Journal of Adhesion, vol 82, issue 6, pp. 555-575 (2006)

1.30.R. Bowling, Journal of The Electrochemical Society, An Analysis of Adhesion on Semiconductor Surfaces, vol 132 , pp. 2208-2214 (1985)

1.31.L. Talbot, R. Cheng, R. Schefer, R. W. and D. Willis, Thermophoresis of Particles in a Heated Boundary Layer, Journal of Fluid Mechanics, 101 , pp. 737-758 (1980)

1.32.G. Zhang, G. Burdick, F. Dai, T. Bibby, and S. Beaudoin, Assessment of Post-CMP Cleaning Mechanisms Using Statistically Designed Experiments, Thin Solid Films, vol 332, pp. 379-384 (1998)

1.33.A. Busnaina, H. Lin, N. Moumen, J. Feng, and J. Taylor, Particle Adhesion and Removal Mechanisms in Post-CMP Cleaning Processes, IEEE Trans. On Semiconductor Manufacturing, vol 15, pp. 374-382 (2002)

1.34.S. Roy, I. Ali, G. Shinn, N. Furusawa, R. Shah, S. Peterman, K. Witt, S. Eastman, and P. Kumar, Post Chemical Mechanical Planarization Cleanup Process for Interlayer Dielectric Films, Journal of The Electrochemical Society, vol 142, pp. 216-226 (1995)

1.35.P. Burggraaf, Wafer Cleaning: Brush and High-Pressure Scrubbers, Semiconductor International, 4, 8, pp. 71-88 (1981)

1.36.J. Bardina, Methods for Surface Particle Removal; A Comparative Study, in Particles on Surfaces 1: Detection, Adhesion, and Removal. K.L. Mittal, Editor Plenum, New York, pp. 329-338 (1988)

1.37.H. Hirano, K. Sato, T. Osaka, H. Kuniyasu, and T. Hattori, A Damage-Free UltraDiluted HF/ $\mathrm{N}_{2}$ Jet Spray for Particle Removal with Minimal Silicon and Oxide Loss, ECS Transactions, vol 1, pp. 3, (2005)

1.38.Y. Kim, H. Cho, and J. Kim, Megasonic Free Single Wafer Cleaning Using Ozone 
Jet Without Pattern Damage and With Minimum Substrate Etching, IEEE Transactions on Semiconductor Manufacturing, vol 17, pp. 3 (2004)

1.39.G. Gale and A. Busnaina, Roles of Cavitation and Acoustic Streaming in Megasonic Cleaning, Particulate Science and Technology, 17, 3, pp. 229 - 238 (1999)

1.40.F. R. Young, Cavitation, McGraw-Hill, London, (1989)

1.41.J. Harman \& E. Lamm, The Impact of Ultrasonic frequency on Particle Removal, Branson Ultrasonics Corp., Precision Processing (2008) http://www.bransoncleaning.com/pdf/Particle.PDF

1.42.K. Muralidharan, M. Keswani, H. Shende, P. Deymier, S. Raghavan, F. Eschbach, A. Sengupta, Experimental and Simulation Investigations of Acoustic Cavitation in Megasonic Cleaning, Proceedings of SPIE, 65171E, (2007)

1.43.K. Bakhtaria, R. Guldiken, P. Makaram, A. Busnaina, J. Park, Experimental and Numerical Investigation of Nanoparticle Removal Using Acoustic Streaming and the Effect of Time, Journal of The Electrochemical Society, 153, 9, pp. G846-G850 (2006)

1.44.A. Busnaina, H. Lin, and N. Moumen, Surface Cleaning Mechanisms and Future Cleaning Requirements, IEEUSEMI Advanced Semiconductor Manufacturing Conference, pp. 328-333 (2000)

1.45.A. Busnaina \& G. Gale, Ultrasonic and Megasonic Particle Removal, Proceedings of Precision Cleaning, pp. 347-360 (1995)

1.46.H. Shende, Megasonic Cleaning in Semi-aqueous and Non-aqueous Systems, MS Thesis (2006)

2.1.H. Kawahara, Ultraclean Surface Processing of Silicon Wafers, Edited by T. Hattori, Springer (1998)

2.2.S. Shwartzman, A. Mayer and W. Kern, Megasonic Particle Removal from Solid State Wafers, RCA Review, vol. 46, pp. 81-105 (1985)

2.3.M. beck, Megasonic Cleaning Charts a Course to the Big Time, Precision Cleaning September (2005)

http://www.prosysmeg.com/technology/articles/precision_cleaning2.php

2.4.J. Rosato, E. Baiya, J. Imonigie, M. Yalamanchili, and E. Hansen, Benefits and Applications of Single Wafer Immersion Wet Processing, Future Fab International, Wafer Processing, issue 13, pp. 236-242 (2002) 
2.5.G. Ferrell and L. Crum, A Novel Cavitation Probe Design and Some Preliminary Measurements of its Application to Megasonic Cleaning, The Journal of Acoustical Society of America, vol 112, 3, pp. 1196-1201 (2002)

2.6.M.Olim, A Theoretical Evaluation of Megasonic Cleaning for Submicron Particles, Journal of the Electrochemistry Society, vol 144, pp. 3657-3659 (1997)

2.7.P. Deymier, A. Khelif, D. Rouhani, J. Vasseur, and S. Raghavan, Theoretical Calculation of the Acoustic Force on a Patterned Silicon Wafer During Megasonic Cleaning, Journal of Applied Physics, vol 88, pp. 5 (2000)

2.8.D. McQueen, Frequency Dependence of Ultrasonic Cleaning, Ultrasonics, vol 24, 5, pp. 273-280, (1986)

2.9.D. McQueen, Ultrasonically Enhanced Chemical Dissociation from Solid Surfaces with Application to Cleaning Electronic Circuit Boards, Ultrasonics, vol 28, 6, pp. 422-427 (1990)

2.10.P. Deymier, J. Vasseur, A. Khelif, D. Rouhani, L. Dobrzynski, and S. Raghavan, Streaming and Removal Forces Due to the Second-Order Sound Field During Megasonic Cleaning of Silicon Wafers, Journal of Applied Physics, vol 88, pp. 11 (2000)

2.11.F. Zhang, A. Busnaina and G. Ahmadi, Particle Adhesion and Removal in Chemical Mechanical Polishing (CMP) and Post-CMP Cleaning, Journal of The Electrochemical Society, vol 146, 7, pp 2665-2669 (1999)

2.12.B. Kanegsberg, Handbook of Critical Cleaning, CRC Press (2001)

2.13.P. Hiemenz and R. Rajagopalan, Principles of Colloid and Surface Chemistry, CRC Press (1997)

2.14.G. Kumar and S. Beaudoin, Undercut Removal of Micrometer-Scale Particles from Surfaces, Journal of The Electrochemical Society, vol 153, 2, pp. G175-G181 (2006)

2.15.B. Derjaguin, Y. Rabinovich, and N. Churaev, Direct Measurement of Molecular Forces, Nature, vol 272, pp. 313-318 (1978)

2.16.H. Ackler, R. French, and Y. Chiang, Comparisons of Hamaker Constant for Ceramic Systems with Intervening Vacuum or Water: From Force Laws and Physical Properties, Journal of Colloid and Interface Science, vol 179, pp. 460-469 (1996) 
2.17.R. Hunter, Foundations of Colloid Science, vol 1, Oxford Science Publications (1987)

2.18.K. Johnson, K. Kendall, and D. Roberts, Proceedings of the Royal Society of London, A, vol 324, pp. 301 (1971)

2.19.D. Maugis and H. Pollock, Surface Forces, Deformation and Adherence at Metal Microcontacts, Acta Metallurgica, vol 32, pp. 1323-1334 (1984)

2.20.B. Derjaguin, V. Muller and Y. Toporov, Effect of Contact Deformations on the Adhesion of Particles, Journal of Colloid Inteface Science, vol 53, pp. 314-325 (1975)

2.21.H. Zhou, M. Gotzinger, and W. Peukert, The influence of Particle Charge and Roughness on Particle-Substrate, Powder Technology, 135-136, pp 82-91 (2003)

2.22.Y. Rabinovich, J. Adler, A. Ata, R. Singh, and B. Moudgil, Adhesion Between Nanoscale Rough Surfaces, Journal of Colloid Interface Science, vol 232, pp. 10-24 (2000)

2.23.M. Ranade, Adhesion and Removal of Fine Particles on Surfaces, Aerosol Science Technology, 7, 2, pp. 161 (1987)

2.24.Prof. Raghavan, MSE 503, Surface Science Class Notes, The University of Arizona (2007) - Online Webpage http://www.mse.arizona.edu/classes/mse503/

2.25.R. Hunter, Zeta Potential in Colloid Science, Principles and Applications, Academic Press (1981)

2.26.J. López-García, M. Aranda-Rascón, and J. Horno, Electrical Double Layer Around a Spherical Colloid Particle: The Excluded Volume Effect, Journal of Colloid and Interface Science, vol 316, pp. 196-201 (2007)

2.27.P. Debye, A Method for the Determination of the Mass of Electrolytic Ions, The Journal of Chemical Physics, vol 1, pp. 13-16 (1933)

2.28.R. Zana and E. Yeager, Ultrasonic Vibration Potentials and their Use in the Determination of Ionic Partial Molal Volumes, The Journal of Physical Chemistry, 71, 13, pp. 521-535 (1967)

2.29.S. Vidal, J. Simonin, and P. Turq, Acoustophoresis Revisited. 1. Electrolyte Solutions, The Journal of Physical Chemistry, 99, pp. 6733-6738 (1995) 
2.30.Hermans, J, Charged Colloid Particles in an Ultrasonic Field, Philosophical Magazine, 25, pp. 426 (1938)

2.31.Hermans, J, Charged Colloid Particles in an Ultrasonic Field-II Particles Surrounded by a Thin Double Layer, Philosophical Magazine, 25, pp. 674 (1938)

2.32.Rutgers, A, Bemerkung, zu den von Ultraschallwellen hervorgerufenen Potentialdifferenzen in Losungen, Physica, 5, 46 (1938)

2.33.B. Marlow, D. Fairhurst, and H. Pendse, Colloid Vibration Potential and the Electrokinetic Characterization of Concentrated Colloids, Langmuir, 4, pp. 611-626 (1988)

2.34.J. Dufreche, O. Bernard, and P. Turq, Transport in Electrolyte Solutions: Are Ions Brownian Particles ?, Journal of Molecular Liquids, 118, pp. 189-194 (2005)

2.35.B. Conway, Modern Aspects of Electrochemistry, vol 14, chapter 1, Springer (2005)

2.36.J. J. Enderby, On Electrical Effects Due to Sound Waves in Colloidal Suspensions, Proceedings of the Royal Society of London, Series A, Mathematical and Physical Sciences, pp. 329-342 (1951)

2.37.D. Pollock, Physical Properies of Materials for Engineers, $2^{\text {nd }}$ edition, CRC Press, pp. 502 (1993)

2.38.H. Ohshima, Colloid vibration potential in a suspension of spherical colloidal particles, Colloids and Surfaces B: Biointerfaces, 56, pp. 6-18 (2007)

2.39.E. Yeager, H. Dietrick, H. Hovorka, Ultrasonic Waves and Electrochemistry. II. Colloidal and Ionic Vibration Potentials, The Journal of the Acoustical Society of America, 25, 3, pp. 456-460 (1953)

2.40.Y. Hozumi, and K. Furusawa, Electrokinetic study on concentrated suspensions using colloid vibration potential measurements, Colloid and Polymer Science, 268, pp. 469-475 (1990)

2.41.J. Cheeke, Fundamentals and Applications of Ultrasonic Waves, CRC Series in Pure and Applied Physics (2002)

2.42.L. Spitzer, P. Bergmann, P. Frank, A. Yaspan, and C. Herring, Physic of Sound in the Sea, Solar Analysis Group (1945)

2.43.G. Ter Haar, Interaction Mechanisms: Non-thermal, Non-cavitational Effects, 
Ultrasound: Medical Applications, Biological Effects, and Hazard Potential, edited by M. Repacholi, M. Grandolfo and A. Rindi, Plenum Press, New York, (1987)

2.44.R. Apfel, Sonic effervescence: A tutorial on acoustic cavitation, Journal of Acoustical Society of America, 101 (3), pp. 1227-1237, March (1997)

2.45.M. Volmer and A. Weber, Nuclei Formation in Supersaturated States, Z. Phys. Chem. 119, pp. 277-301 (1926)

2.46.W. Doring, Z. Phys. Chem, 36, pp. 371-386 (1937)

2.47.W. Coakley and W. Nyborg, Ultrasound: Its applications in Medicine and Biology, edited by F. Fry, Elsevier Press, pp. 109 (1978)

2.48.F. Young, Cavitation, McGraw-Hill Company Limited (1989)

2.49.W. Lauterborn, Numerical investigation of nonlinear oscillations of gas bubbles in liquids, The Journal of The Acoustical Society of America, vol 59, pp. 283-293 (1976)

2.50.H. Flynn, Physical Acoustics, vol 1B, edited by W. Mason, Academic Press, Chapter 9, pp. 85 (1964)

2.51.E. Neppiras and B. Noltingk, Cavitation Produced by Ultrasonics : Theoretical Conditions for the Onset of Cavitation, Proceedings of Physical Society B (London), vol 64 B, pp. 1032-1038 (1951)

2.52.Holland, $\mathrm{C}$, Thresholds of transient cavitation produced by pulsed ultrasound in a controlled nuclei environment, $\mathrm{PhD}$ dissertation, Yale University (1989)

2.53.R. Apfel, Methods of Experimental Physics, vol 19, Ultrasonics, Academic Press, pp. 373 (1981)

2.54.B. Noltingk and E. Neppiras, Cavitation Produced by Ultrasonics, Proceedings of Physical Society B (London), vol 63 B, pp. 674-685 (1950)

2.55.R. Apfel, Possibility of Cavitation from diagnostic ultrasound, IEEE Transactions on Ultrasonics, Ferroelectrics and Frequency Control, vol UFFC 34, 2, March (1986)

2.56.E. Neppiras, Acoustic Cavitation, Phys. Rep., 61, pp. 160-251 (1980)

2.57.E. Neppiras, Acoustic Cavitation Thresholds and Cyclic Processes, Ultrasonics, 18, pp. 201-209 (1980) 
2.58.C. Ohl, T. Kurz, R. Geisler, O. Lindau, W. Lauterborn, Bubble Dynamics, Shock Waves and Sonoluminescence, Philos. Trans. Roy. Soc. A 357, pp. 269 (1999)

2.59.A. Philip and W. Lauterborn, Cavitation Erosion by Single Laser Produced Bubbles, Journal of Fluid Mechanics, 361, pp. 75 (1998)

2.60.V. Minsier, J. Proost, Shock wave emission upon spherical bubble collapse during cavitation induced megasonic surface cleaning, Ultrason. Sonochem., In Press (2007)

2.61.M. Plesset and R. Chapman, Collapse of an initially spherical cavity in the neigbourhood of a solid boundary, The Journal of Fluid Mechanics, vol 47, 2, pp. 283-290 (1971)

2.62.A. Walton and G. Reynolds, Sonoluminescence, Advances in Physics, vol 33, 6, pp. 595-660 (1984)

2.63.B. Dey, and S. Aubry, New Suggestion concerning the origin of sonoluminescence, Physica, D 216, pp. 136-156 (2006)

2.64.C. Sehgal, R. Sutherland \& R. Verrall, Optical Spectra of Sonoluminescence from Transient and Stable Cavitation in Water Saturated with Various Gases, The Journal of Physical Chemistry, vol 84, 4, pp. 388-395 (1980)

2.65.V. Griffing, The Chemical Effect of Ultrasonics, J. Chem. Phys, 20, pp. 939-942 (1952)

2.66.V. Griffing and D. Sette, Phys. rev, 87, pp. 234-240 (1952)

2.67.T. Saxena and W. Nyborg, J. Chem. Phys, 53, 1722 (1970)

2.68.E. Flint and K. Suslick, Sonoluminescence from non-aqueous liquids: emission from small molecules, The Journal of American Chemical Society, 111, 6987 (1989)

2.69.Y. Didenko, W. McNamara and K. Suslick, Hot Spot Conditions during Cavitation in Water, The Journal of American Chemical Society, 121, pp5817-5818 (1999)

2.70.M. Faraday, Philosophical Transactions, vol 121, pp 229 (1831)

2.71.L. Rayleigh, Philisophcal Transactions, vol 175, pp 1 (1884)

2.72.L. Rayleigh, Theory of Sound, Bover Publications, New York (1945)

2.73.C. Lee and T. Wang, Outer Acosutic Streaming, The Journal of The Acoustical 
Society of America, vol 88, 5, pp. 2367 (1990)

2.74.L. Rozenberg, High Intensity Ultrasonic Fields, Plenum Press, New York - London (1971)

2.75.W. Nyborg, Acoustic Streaming due to Attenuated Plane Waves, The Journal of The Acoustical Society of America, vol 25, 1, pp. 68 (1953)

2.76.D. Zhang, Fundamental Study of Megasonic Cleaning, PhD Thesis, University of Minnesota (1993)

2.77.L. Kuznetsova and W. Coakley, Applications of ultrasound streaming and radiation force in biosensors, Biosesors and Bioelectronics, vol 22, 8, pp. 1567-1577 (2007)

3.1.M. Keswani, S. Raghavan, S. Verhaverbeke, K. Muralidharan, and P. Deymier, Effect of Solution Chemistry in Megasonic Cleaning, Surface Preparation and Cleaning Symposium, SEMATECH, Austin, TX (2007)

3.2.M. Keswani, S. Raghavan, and P. Deymier, Effect of Ionic Strength in Megasnic Cleaning - Possible Role of Electro-acoustic Effects, Austin, TX (2008)

3.3.V. Pandit, M. Keswani, S. Raghavan, K. Muralidharan, P. Deymier, F. Eshbach, A. Sengupta, H. Yun, Removal of Gold Particles from Chromium Oxynitride Surface using Dilute Sulfuric Acid Solutions, ECS Transactions, 11, 2, pp. 471 (2007)

3.4.Product Systems Inc., MicroProbe System, Cavitation Monitor - User's Guide, www.prosysmeg.com

4.1.A. Kumar, Speed of Sound in Concentrated Aqueous $\mathrm{KCl}$ Solutions from $278.15 \mathrm{~K}$ to 338.15 K, J. Chem. Eng. Data, 48, pp. 388-391 (2003)

4.2.R. Apfel, The Journal of The Acoustical Society of America, vol 69, pp 1624 (1981)

4.3.A. Catenaccio, Y. Daruich, and C. Magallanes, Temperature Dependence of the Permittivity of Water, Chemical Physics Letters, vol 367, pp. 669-671 (2003)

4.4.A. Revil, P. Pezard, and P. Glover, Streaming Potential in Porous Media 1. Theory of the Zeta Potential, Journal of Geophysical Research, vol 104, no B9, pp. 2002120031 (1999)

4.5.P. Somasundaran and R. Kulkani, A New Streaming Potential Apparatus and Study of Temperature Using it, Journal of Colloid Interface Science, vol 45, pp. 591-600 (1973) 
4.6.T. Ishido, and H. Mizutani, Experimental and Theoretical Basis of Electrokinetic Phenomena in Rock-Water Systems and its Applications to Geophysics, Journal of Geophysical Research, vol 86, pp. 1763-1775 (1981)

4.7.B. E. Conway, Electrochemical Data, Greenwood Press, pp. 144-145 (1969)

4.8.K. Lee and S. Raghavan, Etch Rates of Silicon and Silicon Dioxide in Ammonia Peroxide Solutions Measured by Quartz Crystal Microbalance Technique, Electrochemical and Solid State State Letters, vol 2, 4, pp. 172-174 (1999) 